\title{
Double crystals of binary and integral matrices
}

\author{
Marc A. A. van Leeuwen \\ Université de Poitiers, Département de Mathématiques, \\ BP 30179, 86962 Futuroscope Chasseneuil Cedex, France \\ Marc .van-Leeuwen@math . univ-poitiers.fr \\ http://www-math.univ-poitiers.fr/〜maavl/
}

Submitted: May 16, 2006; Accepted: Oct 2, 2006; Published: Oct 12, 2006

Mathematics Subject Classifications: 05E10, 05E15

\begin{abstract}
We introduce a set of operations that we call crystal operations on matrices with entries either in $\{0,1\}$ or in $\mathbf{N}$. There are horizontal and vertical forms of these operations, which commute with each other, and they give rise to two different structures of a crystal graph of type $A$ on these sets of matrices. They provide a new perspective on many aspects of the RSK correspondence and its dual, and related constructions. Under a straightforward encoding of semistandard tableaux by matrices, the operations in one direction correspond to crystal operations applied to tableaux, while the operations in the other direction correspond to individual moves occurring during a jeu de taquin slide. For the (dual) RSK correspondence, or its variant the Burge correspondence, a matrix $M$ can be transformed by horizontal respectively vertical crystal operations into each of the matrices encoding the tableaux of the pair associated to $M$, and the inverse of this decomposition can be computed using crystal operations too. This decomposition can also be interpreted as computing Robinson's correspondence, as well as the Robinson-Schensted correspondence for pictures. Crystal operations shed new light on the method of growth diagrams for describing the RSK and related correspondences: organising the crystal operations in a particular way to determine the decomposition of matrices, one finds growth diagrams as a method of computation, and their local rules can be deduced from the definition of crystal operations. The Schützenberger involution and its relation to the other correspondences arise naturally in this context. Finally we define a version of Greene's poset invariant for both of the types of matrices considered, and show directly that crystal operations leave it unchanged, so that for such questions in the setting of matrices they can take play the role that elementary Knuth transformations play for words.
\end{abstract}




\section{Introduction}

\section{$\S 0$. Introduction.}

The Robinson-Schensted correspondence between permutations and pairs of standard Young tableaux, and its generalisation by Knuth to matrices and semistandard Young tableaux (the RSK correspondence) are intriguing not only because of their many surprising combinatorial properties, but also by the great variety in ways in which they can be defined. The oldest construction by Robinson was (rather cryptically) defined in terms of transformations of words by "raising" operations. The construction by Schensted uses the well known insertion of the letters of a word into a Young tableau. While keeping this insertion procedure, Knuth generalised the input data to matrices with entries in $\mathbf{N}$ or in $\{0,1\}$. He also introduced a "graph theoretical viewpoint" (which could also be called poset theoretical, as the graph in question is the Hasse diagram of a finite partially ordered set) as an alternative construction to explain the symmetry of the correspondence; a different visualisation of this construction is presented in the "geometrical form" of the correspondence by Viennot, and in Fulton's "matrix-ball" construction. A very different method of describing the correspondence can be given using the game of "jeu de taquin" introduced by Lascoux and Schützenberger. Finally a construction for the RSK correspondence using "growth diagrams" was given by Fomin; it gives a description of the correspondence along the same lines as Knuth's graph theoretical viewpoint and its variants, but it has the great advantage of avoiding all iterative modification of data, and computes the tableaux directly by a double induction along the rows and columns of the matrix.

The fact that these very diverse constructions all define essentially the same correspondence (or at least correspondences that are can be expressed in terms of each other in precise ways) can be shown using several notions that establish bridges between them. For instance, to show that the "rectification" process using jeu de taquin gives a well defined map that coincides with the one defined by Schensted insertion, requires (in the original approach) the additional consideration of an invariant quantity for jeu de taquin (a special case of Greene's invariant for finite posets), and of a set of elementary transformations of words introduced by Knuth. A generalisation of the RSK correspondence from matrices to "pictures" was defined by Zelevinsky, which reinforces the link with Littlewood-Richardson tableaux already present in the work of Robinson; it allows the correspondences considered by Robinson, Schensted, and Knuth to be viewed as derived from a single general correspondence. The current author has shown that this correspondence can alternatively be described using (two forms of) jeu de taquin for pictures instead of an insertion process, and that in this approach the use of Greene's invariant and elementary Knuth operations can be avoided. A drawback of this point of view is that the complications of the Littlewood-Richardson rule are built into the notion of pictures itself; for understanding that rule we have also given a description that is simpler (at the price of losing some symmetry), where semistandard tableaux replace pictures, and "crystal" raising and lowering operations replace one of the two forms of jeu de taquin, so that Robinson's correspondence is completely described in terms of jeu de taquin and crystal operations.

In this paper we shall introduce a new construction, which gives rise to correspondences that may be considered as forms of the RSK correspondence (and of variants 
of it). Its interest lies not so much in providing yet another computational method for that correspondence, as in giving a very simple set of rules that implicitly define it, and which can be applied in sufficiently flexible ways to establish links with nearly all known constructions and notions related to it. Simpler even than semistandard tableaux, our basic objects are matrices with entries in $\mathbf{N}$ or in $\{0,1\}$, and the basic operations considered just move units from one entry to an adjacent entry. As the definition of those operations is inspired by crystal operations on words or tableaux, we call them crystal operations on matrices.

Focussing on small transformations, in terms of which the main correspondences arise only implicitly and by a non-deterministic procedure, our approach is similar to that of jeu de taquin, and to some extent that of elementary Knuth transformations. By comparison our moves are even smaller, they reflect the symmetry of the RSK correspondence, and they can be more easily related to the constructions of that correspondence by Schensted insertion or by growth diagrams. Since the objects acted upon are just matrices, which by themselves hardly impose any constraints at all, the structure of our construction comes entirely from the rules that determine when the transfer of a unit between two entries is allowed. Those rules, given in definitions 1.3.1 and 1.4.1 below, may seem somewhat strange and arbitrary; however, we propose to show in this paper is that in many different settings they do precisely the right thing to allow interesting constructions. One important motivating principle is to view matrices as encoding semistandard tableaux, by recording the weights of their individual rows or columns; this interpretation will reappear all the time. All the same it is important that we are not forced to take this point of view: sometimes it is clearest to consider matrices just as matrices.

While the above might suggest that we introduced crystal operations in an attempt to find a unifying view to the numerous approaches to the RSK correspondence, this paper in fact originated as a sequel to [vLee5], at the end of which paper we showed how two combinatorial expressions for the scalar product of two skew Schur functions, both equivalent to Zelevinsky's generalisation of the Littlewood-Richardson rule, can be derived by applying cancellations to two corresponding expressions for these scalar products as huge alternating sums. We were led to define crystal operations in an attempt to organise those cancellations in such a way that they would respect the symmetry of those expressions with respect to rows and columns. We shall remain faithful to this original motivation, by taking that result as a starting point for our paper; it will serve as motivation for the precise form of the definition crystal operations on matrices. That result, and the Littlewood-Richardson rule, do not however play any role in our further development, so the reader may prefer to take the definitions of crystal operations as a starting point, and pick up our discussion from there.

This paper is a rather long one, even by the author's standards, but the reason is not that our constructions are complicated or that we require difficult proofs in order to justify them. Rather, it is the large number of known correspondences and constructions for which we wish to illustrate the relation with crystal operations that accounts for much of the length of the paper, and the fact that we wish to describe those relations precisely rather than in vague terms. For instance, we arrive fairly easily at our 


\subsection{Notations}

central theorem 3.1.3, which establishes the existence of bijective correspondences with the characteristics of the RSK correspondence and its dual; however, a considerable additional analysis is required to identify these bijections precisely in terms of known correspondences, and to prove the relation found. Such detailed analyses require some hard work, but there are rewards as well, since quite often the results have some surprising aspects; for instance the correspondences of the mentioned theorem turn out to be more naturally described using column insertion than using row insertion, and in particular we find for integral matrices the Burge correspondence rather than the RSK correspondence. We do eventually find a way in which the RSK correspondence arises directly from crystal operations, in proposition 4.4.4, but this is only after exploring various different possibilities of constructing growth diagrams.

Our paper is organised as follows. We begin directly below by recalling from [vLee5] some basic notations that will be used throughout. In $\S 1$ we introduce, first for matrices with entries in $\{0,1\}$ and then for those with entries in $\mathbf{N}$, crystal operations and the fundamental notions related to them, and we prove the commutation of horizontal and vertical operations, which will be our main technical tool. In $\S 2$ we mention a number of properties of crystal graphs, which is the structure one obtains by considering only vertical or only horizontal operations; in this section we also detail the relation between crystal operations and jeu de taquin. In $\S 3$ we start considering double crystals, the structure obtained by considering both vertical and horizontal crystal operations. Here we construct our central bijective correspondence, which amounts to a decomposition of every double crystal as a Cartesian product of two simple crystals determined by one same partition, and we determine how this decomposition is related to known Knuth correspondences. In $\S 4$ we present the most novel aspect of the theory of crystal operations on matrices, namely the way in which the rules for such operations lead to a method of computing the decomposition of theorem 3.1.3 using growth diagrams. The use of growth diagrams to compute Knuth correspondences is well known of course, but here the fact that such a growth diagram exists, and the local rule that this involves, both follow just from elementary properties of crystal operations, without even requiring enumerative results about partitions. In $\S 5$ we study the relation between crystal operations and the equivalents in terms of matrices of increasing and decreasing subsequences, and more generally of Greene's partition-valued invariant for finite posets. Finally, in $\S 6$ we provide the proofs of some results, which were omitted in text of the preceding sections to avoid distracting too much from the main discussion. (However for all our central results the proofs are quite simple and direct, and we have deemed it more instructive to give them right away in the main text.)

\subsection{Notations.}

We briefly review those notations from [vLee5] that will be used in the current paper. We shall use the Iverson symbol, the notation [ condition] designating the value 1 if the Boolean condition holds and 0 otherwise. For $n \in \mathbf{N}$ we denote by $[n]$ the set $\{i \in \mathbf{N} \mid i<n\}=\{0, \ldots, n-1\}$. The set $\mathcal{C}$ of compositions consists of the sequences $\left(\alpha_{i}\right)_{i \in \mathbf{N}}$ with $\alpha_{i} \in \mathbf{N}$ for all $i$, and $\alpha_{i}=0$ for all but finitely many $i$; it may be thought of as $\bigcup_{n \in \mathbf{N}} \mathbf{N}^{n}$ where each $\mathbf{N}^{n}$ is considered as a subset of $\mathbf{N}^{n+1}$ by extension 
of its vectors by an entry 0 . Any $\alpha \in \mathcal{C}$ is said to be a composition of the number $|\alpha|=\sum_{i \in \mathbf{N}} \alpha_{i}$. We put $\mathcal{C}^{[2]}=\left\{\alpha \in \mathcal{C} \mid \forall i: \alpha_{i} \in[2]\right\}$; its elements are called binary compositions. The set $\mathcal{P} \subset \mathcal{C}$ of partitions consists of compositions that are weakly decreasing sequences. The operators ' + ' and '-', when applied to compositions or partitions, denote componentwise addition respectively subtraction.

The diagram of $\lambda \in \mathcal{P}$, which is a finite order ideal of $\mathbf{N}^{2}$, is denoted by $[\lambda]$, and the conjugate partition of $\lambda$ by $\lambda^{t}$. For $\kappa, \lambda \in \mathcal{P}$ the symbol $\lambda / \kappa$ is only used when $[\kappa] \subseteq[\lambda]$ and is called a skew shape; its diagram $[\lambda / \kappa]$ is the set theoretic difference $[\lambda]-[\kappa]$, and we write $|\lambda / \mu|=|\lambda|-|\mu|$. For $\alpha, \beta \in \mathcal{C}$ the relation $\alpha-\beta$ is defined to hold whenever one has $\beta_{i+1} \leq \alpha_{i} \leq \beta_{i}$ for all $i \in \mathbf{N}$; this means that $\alpha, \beta \in \mathcal{P}$, that $[\alpha] \subseteq[\beta]$, and that $[\beta / \alpha]$ has at most one square in any column. When $\mu-\lambda$, the skew shape $\lambda / \mu$ is called a horizontal strip. If $\mu^{t}-\lambda^{t}$ holds, we call $\lambda / \mu$ a vertical strip and write $\mu<\lambda$; this condition amounts to $\mu, \lambda \in \mathcal{P}$ and $\lambda-\mu \in \mathcal{C}^{[2]}$ (so $[\lambda / \mu]$ has at most one square in any row).

A semistandard tableau $T$ of shape $\lambda / \kappa$ (written $T \in \operatorname{SST}(\lambda / \kappa)$ ) is a sequence $\left(\lambda^{(i)}\right)_{i \in \mathbf{N}}$ of partitions starting at $\kappa$ and ultimately stabilising at $\lambda$, of which successive members differ by horizontal strips: $\lambda^{(i)}-\lambda^{(i+1)}$ for all $i \in \mathbf{N}$. The weight $\operatorname{wt}(T)$ of $T$ is the composition $\left(\left|\lambda^{(i+1)} / \lambda^{(i)}\right|\right)_{i \in \mathbf{N}}$. Although we shall work mostly with such tableaux, there will be situations where it is more natural to consider sequences in which the relation between successive members is reversed $\left(\lambda^{(i)} \rightarrow \lambda^{(i+1)}\right)$ or transposed $\left(\lambda^{(i)} \leftarrow \lambda^{(i+1)}\right)$, or both $\left(\lambda^{(i)} \neg \lambda^{(i+1)}\right)$; such sequences will be called reverse and/or transpose semistandard tableaux. The weight of transpose semistandard tableaux is then defined by the same expression as that of ordinary ones, while for their reverse counterparts it is the composition $\left(\left|\lambda^{(i)} / \lambda^{(i+1)}\right|\right)_{i \in \mathbf{N}}$.

The set $\mathcal{M}$ is the matrix counterpart of $\mathcal{C}$ : it consists of matrices $M$ indexed by pairs $(i, j) \in \mathbf{N}^{2}$, with entries in $\mathbf{N}$ of which only finitely many are nonzero (note that rows and columns are indexed starting from 0). It may be thought of as the union of all sets of finite matrices with entries in $\mathbf{N}$, where smaller matrices are identified with larger ones obtained by extending them with entries 0 . The set of such matrices with entries restricted to $[2]=\{0,1\}$ will be denoted by $\mathcal{M}^{[2]}$; these are called binary matrices. For matrices $M \in \mathcal{M}$, we shall denote by $M_{i}$ its row $i$, which is $\left(M_{i, j}\right)_{j \in \mathbf{N}} \in \mathcal{C}$, while $M_{j}^{t}=$ $\left(M_{i, j}\right)_{i \in \mathbf{N}} \in \mathcal{C}$ denotes its column $j$. We denote by row $(M)=\left(\left|M_{i}\right|\right)_{i \in \mathbf{N}}$ the composition formed by the row sums of $M$, and by $\operatorname{col}(M)=\left(\left|M_{j}^{t}\right|\right)_{j \in \mathbf{N}}$ the composition formed by its column sums, and we define $\mathcal{M}_{\alpha, \beta}=\{M \in \mathcal{M} \mid \operatorname{row}(M)=\alpha, \operatorname{col}(M)=\beta\}$ and $\mathcal{M}_{\alpha, \beta}^{[2]}=\mathcal{M}_{\alpha, \beta} \cap \mathcal{M}^{[2]}$.

In the remainder of our paper we shall treat $\mathcal{M}^{[2]}$ and $\mathcal{M}$ as analogous but separate universes, in other words we shall never consider a binary matrix as an integral matrix whose entries happen to be $\leq 1$, or vice versa; this will allow us to use the same notation for analogous constructions in the binary and integral cases, even though their definition for the integral case is not an extension of the binary one. 


\section{Crystal operations on matrices}

\section{$\S 1$. Crystal operations on matrices.}

The motivation and starting point for this paper are formed by a number of expressions for the scalar product between two Schur functions in terms over enumerations of matrices, which were described in [vLee5]. To present them, we first recall the way tableaux were encoded by matrices in that paper.

\subsection{Encoding of tableaux by matrices.}

A semistandard tableau $T=\left(\lambda^{(i)}\right)_{i \in \mathbf{N}}$ of shape $\lambda / \kappa$ can be displayed by drawing the diagram $[\lambda / \kappa]$ in which the squares of each strip $\left[\lambda^{(i+1)} / \lambda^{(i)}\right]$ are filled with entries $i$. Since the columns of such a display are strictly increasing and the rows weakly increasing, such a display is uniquely determined by its shape plus one of the following two informations: (1) for each column $C_{j}$ the set of entries of $C_{j}$, or (2) for each row $R_{i}$ the multiset of entries of $R_{i}$. Each of those informations can be recorded in a matrix: the binary matrix $M \in \mathcal{M}^{[2]}$ in which $M_{i, j} \in\{0,1\}$ indicates the absence or presence of an entry $i$ in column $C_{j}$ of the display of $T$ will be called the binary encoding of $T$, while the integral matrix $N \in \mathcal{M}$ in which $N_{i, j}$ gives the number of entries $j$ in row $R_{i}$ of the display of $T$ will be called the integral encoding of $T$. In terms of the shapes $\lambda^{(i)}$ these matrices can be given directly by $M_{i}=\left(\lambda^{(i+1)}\right)^{t}-\left(\lambda^{(i)}\right)^{t}$ for all $i$ and $N_{j}^{t}=\left(\lambda^{(j+1)}\right)-\left(\lambda^{(j)}\right)$ for all $j$, cf. [vLee5, definition 1.2.3]. Note that the columns $M_{j}^{t}$ of the binary encoding correspond to the columns $C_{j}$, and the rows $N_{i}$ of the integral encoding to the rows $R_{i}$. While this facilitates the interpretation of the matrices, it will often lead to an interchange of rows and columns between the binary and integral cases; for instance from the binary encoding $M$ the weight wt $(T)$ can be read off as row $(M)$, while in terms of the integral encoding $N$ it is $\operatorname{col}(N)$. Here is an example of the display of a semistandard tableau $T$ of shape $(9,8,5,5,3) /(4,1)$ and weight $(2,3,3,2,4,4,7)$, with its binary and integral encodings $M$ and $N$, which will be used in examples throughout this paper:

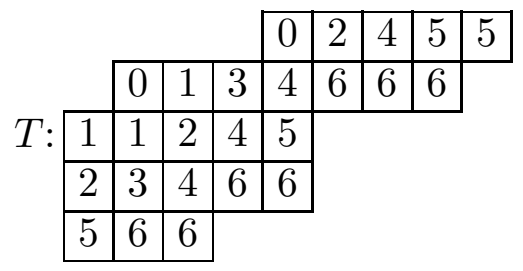

$$
M:\left(\begin{array}{ccccccccc}
0 & 1 & 0 & 0 & 1 & 0 & 0 & 0 & 0 \\
1 & 1 & 1 & 0 & 0 & 0 & 0 & 0 & 0 \\
1 & 0 & 1 & 0 & 0 & 1 & 0 & 0 & 0 \\
0 & 1 & 0 & 1 & 0 & 0 & 0 & 0 & 0 \\
0 & 0 & 1 & 1 & 1 & 0 & 1 & 0 & 0 \\
1 & 0 & 0 & 0 & 1 & 0 & 0 & 1 & 1 \\
0 & 1 & 1 & 1 & 1 & 1 & 1 & 1 & 0
\end{array}\right), \quad N:\left(\begin{array}{ccccccc}
1 & 0 & 1 & 0 & 1 & 2 & 0 \\
1 & 1 & 0 & 1 & 1 & 0 & 3 \\
0 & 2 & 1 & 0 & 1 & 1 & 0 \\
0 & 0 & 1 & 1 & 1 & 0 & 2 \\
0 & 0 & 0 & 0 & 0 & 1 & 2
\end{array}\right) .
$$

To reconstruct $T$ from its binary or integral encoding, one needs to know the shape $\lambda / \kappa$ of $T$, which is not recorded in the encoding; since $\lambda$ and $\kappa$ are related by 


\subsection{Encoding of tableaux by matrices}

$\lambda^{t}-\kappa^{t}=\operatorname{col}(M)$ in the binary case and by $\lambda-\kappa=\operatorname{row}(N)$ in the integral case, it suffices to know one of them. Within the sets $\mathcal{M}^{[2]}$ and $\mathcal{M}$ of all binary respectively integral matrices, each shape $\lambda / \kappa$ defines a subset of matrices that occur as encodings of tableaux of that shape: we denote by $\operatorname{Tabl}^{[2]}(\lambda / \kappa) \subseteq \mathcal{M}^{[2]}$ the set of binary encodings of tableaux $T \in \operatorname{SST}(\lambda / \kappa)$, and by $\operatorname{Tabl}(\lambda / \kappa) \subseteq \mathcal{M}$ the set of integral encodings of such tableaux. The conditions that define such subsets, which we shall call "tableau conditions", can be stated explicitly as follows.

1.1.1. Proposition. Let $\lambda / \kappa$ be a skew shape. For $M \in \mathcal{M}^{[2]}, M \in \operatorname{Tabl}^{[2]}(\lambda / \kappa)$ holds if and only if $\operatorname{col}(M)=\lambda^{t}-\kappa^{t}$, and $\kappa^{t}+\sum_{i<k} M_{i} \in \mathcal{P}$ for all $k \in \mathbf{N}$. For $M \in \mathcal{M}$ one has $M \in \operatorname{Tabl}(\lambda / \kappa)$ if and only if $\operatorname{row}(M)=\lambda-\kappa$, and $\left(\kappa+\sum_{j<l} M_{j}^{t}\right)-\left(\kappa+\sum_{j \leq l} M_{j}^{t}\right)$ for all $l \in \mathbf{N}$.

Proof. This is just a verification that an appropriate tableau encoded by the matrix can be reconstructed if and only if the given conditions are satisfied. We have seen that if $M \in \mathcal{M}^{[2]}$ is the binary encoding of some $\left(\lambda^{(i)}\right)_{i \in \mathbf{N}} \in \operatorname{SST}(\lambda / \kappa)$, then $M_{i}=$ $\left(\lambda^{(i+1)}\right)^{t}-\left(\lambda^{(i)}\right)^{t}$ for all $i$, which together with $\lambda^{(0)}=\kappa$ implies $\left(\lambda^{(k)}\right)^{t}=\kappa^{t}+\sum_{i<k} M_{i}$ for $k \in \mathbf{N}$. A sequence of partitions $\lambda^{(i)}$ satisfying this condition exists if and only if each value $\kappa^{t}+\sum_{i<k} M_{i}$ is a partition. If so, each condition $\lambda^{(i)}-\lambda^{(i+1)}$ will be automatically satisfied, since it is equivalent to $\left(\lambda^{(i)}\right)^{t} \leftarrow\left(\lambda^{(i+1)}\right)^{t}$, while by construction $\left(\lambda^{(i+1)}\right)^{t}-\left(\lambda^{(i)}\right)^{t}=M_{i} \in \mathcal{C}^{[2]}$; therefore $\left(\lambda^{(i)}\right)_{i \in \mathbf{N}}$ will be a semistandard tableau. Moreover $\operatorname{col}(M)=\lambda^{t}-\kappa^{t}$ means that $\kappa^{t}+\sum_{i<k} M_{i}=\lambda^{t}$ for sufficiently large $k$, and therefore that the shape of the semistandard tableau found will be $\lambda / \kappa$.

Similarly if $M \in \mathcal{M}$ is the integral encoding of some $\left(\lambda^{(i)}\right)_{i \in \mathbf{N}} \in \operatorname{SST}(\lambda / \kappa)$, then we have seen that $M_{j}^{t}=\left(\lambda^{(j+1)}\right)-\left(\lambda^{(j)}\right)$ for all $j$, which together with $\lambda^{(0)}=\kappa$ implies $\lambda^{(l)}=\kappa+\sum_{j<l} M_{j}^{t}$ for $l \in \mathbf{N}$. By definition the sequence $\left(\lambda^{(i)}\right)_{i \in \mathbf{N}}$ so defined for a given $\kappa$ and $M \in \mathcal{M}$ is a semistandard tableau if and only if $\lambda^{(l)}-\lambda^{(l+1)}$ for all $l \in \mathbf{N}$ (which implies that all $\lambda^{(l)}$ are partitions), in other words if and only if $\left(\kappa+\sum_{j<l} M_{j}^{t}\right)-$ $\left(\kappa+\sum_{j \leq l} M_{j}^{t}\right)$ for all $l \in \mathbf{N}$. The value of $\lambda^{(l)}$ ultimately becomes $\kappa+\operatorname{row}(M)$, so the semistandard tableau found will have shape $\lambda / \kappa$ if and only if $\operatorname{row}(M)=\lambda-\kappa$.

Littlewood-Richardson tableaux are semistandard tableaux satisfying some additional conditions, and the Littlewood-Richardson rule expresses certain decomposition multiplicities by counting such tableaux (details, which are not essential for the current discussion, can be found in [vLee3]). In [vLee5, theorems 5.1 and 5.2], a generalised version of that rule is twice stated in terms of matrices, using respectively binary and integral encodings. A remarkable aspect of these formulations is that the additional conditions are independent of the tableau conditions that these matrices must also satisfy, and notably of the shape $\lambda / \kappa$ for which they do so; moreover, the form of those additional conditions is quite similar to the tableau conditions, but with the roles of rows and columns interchanged. We shall therefore consider these conditions separately, and call them "Littlewood-Richardson conditions".

1.1.2. Definition. Let $\nu / \mu$ be a skew shape. The set $\operatorname{LR}^{[2]}(\nu / \mu) \subseteq \mathcal{M}^{[2]}$ is defined by $M \in \operatorname{LR}^{[2]}(\nu / \mu)$ if and only if $\operatorname{row}(M)=\nu-\mu$, and $\mu+\sum_{j \geq l} M_{j}^{t} \in \mathcal{P}$ for all $l \in \mathbf{N}$, 


\subsection{Commuting cancellations}

and the set $\operatorname{LR}(\nu / \mu) \subseteq \mathcal{M}$ is defined by $M \in \operatorname{LR}(\nu / \mu)$ if and only if $\operatorname{col}(M)=\nu-\mu$, and $\left(\mu+\sum_{i<k} M_{i}\right)-\left(\mu+\sum_{i \leq k} M_{i}\right)$ for all $k \in \mathbf{N}$.

Thus for integral matrices, the Littlewood-Richardson conditions for a given skew shape are just the tableau conditions for the same shape, but applied to the transpose matrix. For binary matrices, the relation is as follows: if $M$ is a finite rectangular binary matrix and $M^{\prime}$ is obtained from $M$ by a quarter turn counterclockwise, then viewing $M$ and $M^{\prime}$ as elements of $\mathcal{M}^{[2]}$ by extension with zeroes, one has $M \in \operatorname{LR}^{[2]}(\lambda / \kappa)$ if and only if $M^{\prime} \in \operatorname{Tabl}^{[2]}(\lambda / \kappa)$. Note that rotation by a quarter turn is not a well defined operation on $\mathcal{M}^{[2]}$, but the matrices resulting from the rotation of different finite rectangles that contain all nonzero entries of $M$ are all related by the insertion or removal of some initial null rows, and such changes do not affect membership of any set $\operatorname{Tabl}^{[2]}(\lambda / \kappa)$ (they just give a shift in the weight of the tableaux encoded by the matrices).

\subsection{Commuting cancellations.}

We can now concisely state the expressions mentioned above for the scalar product between two skew Schur functions, which were given in [vLee5]. What interests us here is not so much what these expressions compute, as the fact that one has different expressions for the same quantity. We shall therefore not recall the definition of this scalar product $\left\langle s_{\lambda / \kappa} \mid s_{\nu / \mu}\right\rangle$, but just note that the theorems mentioned above express that value as $\#\left(\operatorname{Tabl}^{[2]}(\lambda / \kappa) \cap \operatorname{LR}^{[2]}(\nu / \mu)\right)$ and as $\#(\operatorname{Tabl}(\lambda / \kappa) \cap \operatorname{LR}(\nu / \mu))$, respectively (the two sets counted encode the same set of tableaux). Those theorems were derived via cancellation from equation [vLee5, (50)], which expresses the scalar product as an alternating sum over tableaux. That equation involves a symbol $\varepsilon(\alpha, \lambda)$, combinatorially defined for $\alpha \in \mathcal{C}$ and $\lambda \in \mathcal{P}$ with values in $\{-1,0,1\}$. For our current purposes the following characterisation of this symbol will suffice: in case $\alpha$ is a partition one has $\varepsilon(\alpha, \lambda)=[\alpha=\lambda]$, and in general if $\alpha, \alpha^{\prime} \in \mathcal{C}$ are related by $\left(\alpha_{i}^{\prime}, \alpha_{i+1}^{\prime}\right)=\left(\alpha_{i+1}-1, \alpha_{i}+1\right)$ for some $i \in \mathbf{N}$, and $\alpha_{j}^{\prime}=\alpha_{j}$ for all $j \notin\{i, i+1\}$, then $\varepsilon(\alpha, \lambda)+\varepsilon\left(\alpha^{\prime}, \lambda\right)=0$ for any $\lambda$. Another pair of equations [vLee5, $(55,54)]$ has an opposite relation to equation [vLee5, (50)], as they contain an additional factor of the form $\varepsilon(\alpha, \lambda)$ in their summand, but they involve neither tableau conditions nor Littlewood-Richardson conditions. These different expressions, stated in the form of summations over all binary or integral matrices but whose range is effectively restricted by the use of the Iverson symbol, and ordered from the largest to the smallest effective range, are as follows. For the binary case they are

$$
\begin{aligned}
\left\langle s_{\lambda / \kappa} \mid s_{\nu / \mu}\right\rangle & =\sum_{M \in \mathcal{M}^{[2]}} \varepsilon\left(\kappa^{t}+\operatorname{col}(M), \lambda^{t}\right) \varepsilon(\mu+\operatorname{row}(M), \nu) \\
& =\sum_{M \in \mathcal{M}^{[2]}}\left[M \in \operatorname{Tabl}^{[2]}(\lambda / \kappa)\right] \varepsilon(\mu+\operatorname{row}(M), \nu) \\
& =\sum_{M \in \mathcal{M}^{[2]}}\left[M \in \operatorname{Tabl}^{[2]}(\lambda / \kappa)\right]\left[M \in \operatorname{LR}^{[2]}(\nu / \mu)\right],
\end{aligned}
$$

and for the integral case 


$$
\begin{aligned}
\left\langle s_{\lambda / \kappa} \mid s_{\nu / \mu}\right\rangle & =\sum_{M \in \mathcal{M}} \varepsilon(\kappa+\operatorname{row}(M), \lambda) \varepsilon(\mu+\operatorname{col}(M), \nu) \\
& =\sum_{M \in \mathcal{M}}[M \in \operatorname{Tabl}(\lambda / \kappa)] \varepsilon(\mu+\operatorname{col}(M), \nu) \\
& =\sum_{M \in \mathcal{M}}[M \in \operatorname{Tabl}(\lambda / \kappa)][M \in \operatorname{LR}(\nu / \mu)]
\end{aligned}
$$

The expressions in (1)-(2)-(3) as well as those in (4)-(5)-(6) are related to one another by successive cancellations: in each step one of the factors $\varepsilon(\alpha, \lambda)$ is replaced by an Iverson symbol that selects only terms for which the mentioned factor $\varepsilon(\alpha, \lambda)$ already had a value 1; this means that all contributions from terms for which that factor $\varepsilon(\alpha, \lambda)$ has been replaced by 0 cancel out against each other.

The symmetry between the tableau conditions and Littlewood-Richardson conditions allows us to achieve the cancellations form (1) to (3) and from (4) to (6) in an alternative way, handling the second factor of the summand first, so that halfway those cancellations one has

$$
\left\langle s_{\lambda / \kappa} \mid s_{\nu / \mu}\right\rangle=\sum_{M \in \mathcal{M}^{[2]}} \varepsilon\left(\kappa^{t}+\operatorname{col}(M), \lambda^{t}\right)\left[M \in \operatorname{LR}^{[2]}(\nu / \mu)\right]
$$

in the binary case, and in the integral case

$$
\left\langle s_{\lambda / \kappa} \mid s_{\nu / \mu}\right\rangle=\sum_{M \in \mathcal{M}} \varepsilon(\kappa+\operatorname{row}(M), \lambda)[M \in \operatorname{LR}(\nu / \mu)] .
$$

Indeed, the cancellation form (1) to (7) is performed just like the one from (1) to (2) would be for matrices rotated a quarter turn (and for $\lambda / \kappa$ in place of $\nu / \mu$ ), while the cancellation form (4) to (8) is performed just like the one from (4) to (5) would be for the transpose matrices. Slightly more care is needed to justify the second cancellation phase, since the Littlewood-Richardson condition in the second factor of the summand does not depend merely on row or column sums, as the unchanging first factor did in the first phase. In the integral case, the second cancellation phase can be seen to proceed like the cancellation from (5) to (6) with matrices transposed, but in the binary case the argument is analogous, but not quite symmetrical to the one used to go from (2) to (3). Of course, we already knew independently of this argument that the right hand sides of (7) and (8) describe the same values as those of (3) and (6).

Although for the two factors of the summand of (1) or (4) we can thus apply cancellations to the summation in either order, and when doing so each factor is in both cases replaced by the same Iverson symbol, the actual method as indicated in [vLee5] by which terms would be cancelled is not the same in both cases. This is so because in the double cancellations leading from (1) to (3) or from (4) to (6), whether passing via (2) respectively (5) or via (7) respectively (8), the first phase of cancellation has rather different characteristics than the second phase. The first phase is a Gessel-Viennot type 


\subsection{Crystal operations for binary matrices}

cancellation; it is general (in that it operates on all terms of the initial summation) and relatively simple (it just needs to make sure that a matrix cancels against one with the same row- or column sums). By contrast the second phase is a Bender-Knuth type cancellation that only operates on terms that have survived the first phase (for matrices satisfying the pertinent tableau condition), and it has to be more careful, in order to assure that whenever such a term is cancelled it does so against a term that also survived the first phase.

The question that motivated the current paper is whether it is possible to find an alternative way of defining the cancellations that has the same effect on the summations (so we only want to change the manner in which cancelling terms are paired up), but which has the property that the cancellation of terms failing one of the (tableau or Littlewood-Richardson) conditions proceeds in the same way, whether it is applied as the first or as the second phase. This requires the definition of each cancellation to be general (in case it is applied first), but also to respect the survival status for the other cancellation (in case it is applied second). The notion of crystal operations on matrices described below will allow us to achieve this goal. We shall in fact see that for instance the cancellation that cancels terms not satisfying the Littlewood-Richardson condition for $\nu / \mu$ is defined independently of the shape $\lambda / \kappa$ occurring in the tableau condition; in fact it respects the validity of the tableau condition for all skew shapes at once.

\subsection{Crystal operations for binary matrices.}

Consider the cancellation of terms that fail the Littlewood-Richardson condition, either going from (2) to (3), or from (1) to (7). Since the condition $M \in \operatorname{LR}^{[2]}(\nu / \mu)$ involves partial sums of all columns of $M$ to the right of a given one, this condition can be tested using a right-to-left pass over the columns of $M$, adding each column successively to composition that is initialised as $\mu$, and checking whether that composition remains a partition. If it does throughout the entire pass, then there is nothing to do, since in particular the final value $\mu+\operatorname{row}(M)$ will be a partition, so that $\varepsilon(\mu+\operatorname{row}(M), \nu)=$ $\left[M \in \mathrm{LR}^{[2]}(\nu / \mu)\right]=[\mu+\operatorname{row}(M)=\nu]$. If on the other hand the composition fails to be a partition at some point, then one can conclude immediately that $M \notin \mathrm{LR}^{[2]}(\nu / \mu)$, so the term for $M$ cancels. Up to this point there is no difference between a GesselViennot type cancellation and a Bender-Knuth type cancellation.

Having determined that the term for $M$ cancels, one must find a matrix $M^{\prime}$ whose term cancels against it. The following properties will hold in all cases. Firstly $M^{\prime}$ will be obtained from $M$ by moving entries within individual columns, so that $\operatorname{col}(M)=$ $\operatorname{col}\left(M^{\prime}\right)$. Secondly, the columns of $M$ that had been inspected at the point where cancellation was detected will be unchanged in $M^{\prime}$, so that the term for $M^{\prime}$ is sure to cancel for the same reason as the one for $M$. Thirdly, a pair of adjacent rows is selected that is responsible for the cancellation; all moves take place between these rows and in columns that had not been inspected, with the effect of interchanging the sums of the entries in those columns between those two rows. In more detail, suppose $\beta$ is the first composition that failed the test to be a partition, formed after including column $l$ (so $\beta=\mu+\sum_{j \geq l} M_{j}^{t}$ ), then there is at least one index $i$ for which $\beta_{i+1}=\beta_{i}+1$; one such $i$ is chosen in a systematic way (for instance the minimal one) 
and all exchanges applied in forming $M^{\prime}$ will be between pairs of entries $M_{i, j}, M_{i+1, j}$ with $j<l$. As a result the partial row sums $\alpha=\sum_{j<l} M_{j}^{t}$ and $\alpha^{\prime}=\sum_{j<l}\left(M^{\prime}\right)_{j}^{t}$ will be related by $\left(\alpha_{i}^{\prime}, \alpha_{i+1}^{\prime}\right)=\left(\alpha_{i+1}, \alpha_{i}\right)$ (the other parts are obviously unchanged), so that $\mu+\operatorname{row}(M)=\alpha+\beta$ and $\mu+\operatorname{row}\left(M^{\prime}\right)=\alpha^{\prime}+\beta$ are related in a way that ensures $\varepsilon(\mu+\operatorname{row}(M), \nu)+\varepsilon\left(\mu+\operatorname{row}\left(M^{\prime}\right), \nu\right)=0$, so that the terms of $M$ and $M^{\prime}$ may cancel out.

Within this framework, there remains some freedom in constructing $M^{\prime}$, and here the Gessel-Viennot and Bender-Knuth types of cancellation differ. If our current cancellation occurs as the first phase, in other words if we are considering the cancellation from (1) to (7), then the fact that we have ensured $\varepsilon(\mu+\operatorname{row}(M), \nu)+\varepsilon\left(\mu+\operatorname{row}\left(M^{\prime}\right), \nu\right)=$ 0 suffices for the cancellation of the terms of $M$ and $M^{\prime}$, and $M^{\prime}$ can simply be constructed by interchanging all pairs of bits $\left(M_{i, j}, M_{i+1, j}\right)$ with $j<l$, which is what the Gessel-Viennot type cancellation does (of course such exchanges only make any difference if the bits involved are unequal). If however our current cancellation occurs as the second phase (so we are considering the cancellation from (2) to (3)), then we must in addition make sure that $M \in \operatorname{Tabl}^{[2]}(\lambda / \kappa)$ holds if and only if $M^{\prime} \in \operatorname{Tabl}^{[2]}(\lambda / \kappa)$ does. This will not in general be the case for the exchange just described, which is why the Bender-Knuth type of cancellation limits the number of pairs of bits interchanged, taking into account the shape $\lambda / \kappa$ for which the tableau condition must be preserved. The (easy) details of how this is done do not concern us here, but we note that among the pairs of unequal bits whose interchange is avoided, there are as many with their bit ' 1 ' in row $i$ as there are with their bit ' 1 ' in row $i+1$, so that the relation between $\beta$ and $\beta^{\prime}$ above is unaffected. The alternative construction given below similarly ensures that $M \in \operatorname{Tabl}^{[2]}(\lambda / \kappa)$ holds if and only if $M^{\prime} \in \operatorname{Tabl}^{[2]}(\lambda / \kappa)$ does, but since it is defined independently of $\lambda / \kappa$, it works for all shapes at once, and it can be applied to any matrix, unlike the Bender-Knuth cancellation which is defined only for (encodings of) tableaux of shape $\lambda / \kappa$.

Our fundamental definition will concern the interchange of a single pair of distinct adjacent bits in a binary matrix; this will be vertical adjacent pair in the discussion above, but for the cancellation of terms failing the tableau condition we shall also use the interchange of horizontally adjacent bits. Our definition gives a condition for allowing such an interchange, which is sufficiently strict that at most one interchange at a time can be authorised between a given pair of adjacent rows or columns and in a given direction (like moving a bit ' 1 ' upwards, which of course also involves a bit ' 0 ' moving downwards). Multiple moves (up to some limit) of a bit in the same direction between the same rows or columns can be performed sequentially, because the matrix obtained after an interchange may permit the interchange of a pair of bits that was not allowed in the original matrix.

1.3.1. Definition. Let $M \in \mathcal{M}^{[2]}$ be a binary matrix.

a. A vertically adjacent pair of bits $\left(M_{k, l}, M_{k+1, l}\right)$ is called interchangeable in $M$ if $M_{k, l} \neq M_{k+1, l}$, and if $\sum_{j=l^{\prime}}^{l-1} M_{k, j} \geq \sum_{j=l^{\prime}}^{l-1} M_{k+1, j}$ for all $l^{\prime} \leq l$, while $\sum_{j=l+1}^{l^{\prime}} M_{k, j} \leq \sum_{j=l+1}^{l^{\prime}} M_{k+1, j}$ for all $l^{\prime} \geq l$.

b. A horizontally adjacent pair of bits $\left(M_{k, l}, M_{k, l+1}\right)$ is called interchangeable in $M$ 


\subsection{Crystal operations for binary matrices}

$$
\begin{aligned}
& \text { if } M_{k, l} \neq M_{k, l+1} \text { and if } \sum_{i=k^{\prime}}^{k-1} M_{i, l} \leq \sum_{i=k^{\prime}}^{k-1} M_{i, l+1} \text { for all } k^{\prime} \leq k \text {, while } \\
& \sum_{i=k+1}^{k^{\prime}} M_{i, l} \geq \sum_{i=k^{\prime}}^{k-1} M_{i, l+1} \text { for all } k^{\prime} \geq k .
\end{aligned}
$$

Applying a upward, downward, leftward, or rightward move to $M$ means interchanging an interchangeable pair of bits, which is respectively of the form $\left(M_{k, l}, M_{k+1, l}\right)=(0,1)$, $\left(M_{k, l}, M_{k+1, l}\right)=(1,0), \quad\left(M_{k, l}, M_{k, l+1}\right)=(0,1)$ or $\left(M_{k, l}, M_{k, l+1}\right)=(1,0)$.

These operations are inspired by crystal (or coplactic) operations, and we shall call them crystal operations on binary matrices. Indeed, horizontal moves correspond to coplactic operations (as defined in [vLee3, §3]) applied to the concatenation of the increasing words with weights given by the (nonzero) rows of $M$, from top to bottom; vertical moves correspond to coplactic operations on the concatenation of increasing words with weights given by the columns of $M$, taken from right to left. Applied to the binary encoding of a semistandard tableau $T$, vertical moves correspond to coplactic operations on $T$.

This definition has a symmetry with respect to rotation of matrices: if a pair of bits in a finite binary matrix is interchangeable, then the corresponding pair of bits in the matrix rotated a quarter turn will also be interchangeable. However the definition does not have a similar symmetry with respect to transposition of matrices, and this makes it a bit hard to memorise. As a mnemonic we draw the matrices $\left(\begin{array}{ll}1 & 0 \\ 0 & 1\end{array}\right)$ and $\left(\begin{array}{ll}0 & 1 \\ 1 & 0\end{array}\right)$ with a line between the pairs of bits that are not interchangeable (and they will not be interchangeable whenever they occur in a $2 \times 2$ submatrix of this form, since the conditions allowing interchange can only get more strict when a matrix is embedded in a larger one); the pairs not separated by a line are in fact interchangeable in the given $2 \times 2$ matrices:

$$
\left(\begin{array}{l|l}
1 & 0 \\
0 & 1
\end{array}\right), \quad\left(\begin{array}{ll}
0 & 1 \\
\hline 1 & 0
\end{array}\right)
$$

As a somewhat larger example, consider vertical moves in the binary matrix

$$
M=\left(\begin{array}{lllllllllllll}
1 & 0 & 0 & 1 & 0 & 1 & 1 & 0 & 0 & 0 & 0 & 0 & 1 \\
0 & 1 & 1 & 1 & 1 & 0 & 0 & 1 & 1 & 0 & 1 & 1 & 1 \\
0 & 1 & 0 & 1 & 0 & 0 & 1 & 1 & 1 & 0 & 1 & 0 & 1
\end{array}\right)
$$

The pair $\left(\begin{array}{l}1 \\ 0\end{array}\right)$ at the top right is interchangeable, because in every initial part of the remainder of rows 0 and 1 , the pairs $\left(\begin{array}{l}0 \\ 1\end{array}\right)$ are at least as numerous as the pairs $\left(\begin{array}{l}1 \\ 0\end{array}\right)$. Since they are in fact always strictly more numerous, the pair $\left(\begin{array}{l}0 \\ 1\end{array}\right)$ in column 1 is also interchangeable (the closest one comes to violating the second inequality in $1.3 .1 \mathrm{a}$ is the equality $\sum_{j=2}^{6} M_{0, j}=3=\sum_{j=2}^{6} M_{1, j}$, and the first inequality poses no problems). None of the remaining pairs in rows 0 and 1 are interchangeable however; for the pair in column 2 the first inequality in 1.3.1a fails for $l^{\prime}=1$ since $M_{0,1}=0 \nsupseteq M_{1,1}=1$, and in fact this inequality continues to fail for $l^{\prime}=1$ and all further columns (often there are other inequalities that fail as well, but one may check that for column 7 the mentioned inequality is the only one that fails). In rows 1 and 2 , only the pair $\left(\begin{array}{l}1 \\ 0\end{array}\right)$ in column 4 
is interchangeable (while all inequalities are also satisfied for columns 5 and 12, these columns contain pairs of equal bits $\left(\begin{array}{l}0 \\ 0\end{array}\right)$ and $\left(\begin{array}{l}1 \\ 1\end{array}\right)$, which are never interchangeable). As an example of successive moves in the same direction, one may check that, in rows 0 and 1 , after interchanging the pair $\left(\begin{array}{l}0 \\ 1\end{array}\right)$ in column 1, one may subsequently interchange similar pairs in columns $7,8,10$, and 11 , in that order.

Let us now show our claim that at most one move at a time is possible between any given pair of rows or columns and in any given direction. Consider the case of adjacent rows, $i, i+1$ and suppose they contain two interchangeable vertically adjacent pairs of bits in columns $j_{0}<j_{1}$. Then one has two opposite inequalities for the range of intermediate columns, which implies that $\sum_{j=j_{0}+1}^{j_{1}-1} M_{i, j}=\sum_{j=j_{0}+1}^{j_{1}-1} M_{i+1, j}$. One can also see that the interchangeable pair in column $j_{0}$ is $\left(\begin{array}{l}1 \\ 0\end{array}\right)$ and the one in column $j_{1}$ is $\left(\begin{array}{l}0 \\ 1\end{array}\right)$, since any other values would contradict definition 1.3.1. So there can be at most one downward move and at most one upward move that can be applied between rows $i$ and $i+1$, with the downward move being to the left of the upward move if both occur. Similarly, at most one leftward move and at most one rightward move can be applied between a given pair of adjacent columns, and if both can, the leftward move is in a row above of the rightward move.

These uniqueness statements justify the following crucially important definition. In keeping with the usual notation for crystal operations, we use the letter $e$ for raising operations and the letter $f$ for lowering operations, but since we have a horizontal and a vertical variant of either one, we attach an arrow pointing in the direction in which the bit ' 1 ' moves.

1.3.2. Definition. (binary raising and lowering operations) Let $M \in \mathcal{M}^{[2]}$.

a. If $M$ contains an interchangeable pair of bits in rows $i$ and $i+1$, then the matrix resulting from the interchange of these bits is denoted by $e_{i}^{\uparrow}(M)$ if the interchange is an upward move, or by $f_{i}^{\downarrow}(M)$ if the interchange is a downward move. If for a given $i \in \mathbf{N}$ the matrix $M$ admits no upward or no downward move interchanging any pair bits in rows $i$ and $i+1$, then the expression $e_{i}^{\uparrow}(M)$ respectively $f_{i}^{\downarrow}(M)$ is undefined.

b. If $M$ contains an interchangeable pair of bits in columns $j$ and $j+1$, then the matrix resulting from the interchange of these bits is denoted by $e_{l}^{\leftarrow}(M)$ if the interchange is a leftward move, or by $f_{l}(M)$ if the interchange is a rightward move. If for a given $j \in \mathbf{N}$ the matrix $M$ admits no leftward or no rightward move interchanging any pair bits in columns $j$ and $j+1$, then the expression $e_{l}^{\leftarrow}(M)$ respectively $f_{l} \rightarrow(M)$ is undefined.

Since an interchangeable pair of bits remains so after it has been interchanged, it follows that whenever $e_{i}^{\uparrow}(M)$ is defined then so is $f_{i}^{\downarrow}\left(e_{i}^{\uparrow}(M)\right)$, and it is equal to $M$. Similarly each of the expressions $e_{i}^{\uparrow}\left(f_{i}^{\downarrow}(M)\right), \quad, e_{j}^{\leftarrow}\left(f_{j}(M)\right)$ and $f_{j}\left(e_{j}^{\leftarrow}(M)\right)$ is defined as soon as its inner application is, in which case it designates $M$. Our next concern will be characterising when expressions such as $e_{i}^{\uparrow}(M)$ are defined, and more generally determining the number of times each of the operations $e_{i}^{\uparrow}, f_{i}^{\downarrow}, e_{j}^{\leftarrow}$ and $f_{j}$ can be successively applied to a given matrix $M$, which we shall call the potential of $M$ for these operations. 


\subsection{Crystal operations for binary matrices}

1.3.3. Definition. For $M \in \mathcal{M}^{[2]}$ and $i, j \in \mathbf{N}$, the numbers $n_{i}^{\uparrow}(M), n_{i}^{\downarrow}(M), n_{j}^{\leftarrow}(M)$, $n_{j}(M) \in \mathbf{N}$ are defined by

$$
\begin{aligned}
n_{i}^{\uparrow}(M) & =\max \left\{\sum_{j \geq l}\left(M_{i+1, j}-M_{i, j}\right) \mid l \in \mathbf{N}\right\}, \\
n_{i}^{\downarrow}(M) & =\max \left\{\sum_{j<l}\left(M_{i, j}-M_{i+1, j}\right) \mid l \in \mathbf{N}\right\}, \\
n_{j}^{\leftarrow}(M) & =\max \left\{\sum_{i<k}\left(M_{i, j+1}-M_{i, j}\right) \mid k \in \mathbf{N}\right\}, \\
n_{j}^{\vec{j}}(M) & =\max \left\{\sum_{i \geq k}\left(M_{i, j}-M_{i, j+1}\right) \mid k \in \mathbf{N}\right\} .
\end{aligned}
$$

1.3.4. Proposition. For $M \in \mathcal{M}^{[2]}$ and $i, j \in \mathbf{N}$, the numbers of times each of $e_{i}^{\uparrow}$, $f_{i}^{\downarrow}, e_{j}^{\leftarrow}$, and $f_{j}$ can be successively applied to $M$ are respectively given by the numbers $n_{i}^{\uparrow}(M), n_{i}^{\downarrow}(M), n_{j}^{\leftarrow}(M)$, and $n_{j}(M)$. Moreover $n_{i}^{\downarrow}(M)-n_{i}^{\uparrow}(M)=\operatorname{row}(M)_{i}-\operatorname{row}(M)_{i+1}$ and $n_{j}(M)-n_{j}^{\leftarrow}(M)=\operatorname{col}(M)_{j}-\operatorname{col}(M)_{j+1}$.

Proof. Suppose $M$ is an $n \times m$ binary matrix (so all entries outside that rectangle are zero) that admits an upward move interchanging a pair of bits $\left(\begin{array}{l}0 \\ 1\end{array}\right)$ in column $l$ of rows $i, i+1$. Then it follows from $\sum_{j=l+1}^{m-1}\left(M_{i+1, j}-M_{i, j}\right) \geq 0$ that $n_{i}^{\uparrow}(M) \geq \sum_{j \geq l}\left(M_{i+1, j}-M_{i, j}\right)>0$. Conversely if $n_{i}^{\uparrow}(M)>0$, then let $l<m$ be the maximal index for which the maximal value of $\sum_{j \geq l}\left(M_{i+1, j}-M_{i, j}\right)$ is attained. One then verifies that $M$ admits an upward move in column $l$ of rows $i, i+1$ : the fact that the pair in that position is $\left(\begin{array}{l}0 \\ 1\end{array}\right)$ follows from the maximality of $l$, and failure of one of the inequalities in 1.3.1a would respectively give a value $l^{\prime}<l$ for which a strictly larger sum is obtained, or a value $l^{\prime}+1>l$ for which a weakly larger sum is obtained, either of which contradicts the choice of $l$.

The statement concerning $e_{i}^{\uparrow}$ can now be proved by induction on $n_{i}^{\uparrow}(M)$. For $n_{i}^{\uparrow}(M)=0$ we have just established that no upward moves in rows $i, i+1$ are possible. So suppose $n_{i}^{\uparrow}(M)>0$ and let $M^{\prime}$ be obtained from $M$ by an upward move in column $l_{0}$. Then replacing $M$ by $M^{\prime}$ decreases the sums $\sum_{j \geq l}\left(M_{i+1, j}-M_{i, j}\right)$ by 2 for all $l \leq l_{0}$, while those sums are unchanged for $l>l_{0}$. The sums for $l \leq l_{0}$ therefore become at most $n_{i}^{\uparrow}(M)-2$, while the sums for $l>l_{0}$ remain at most $n_{i}^{\uparrow}(M)-1$ (since $l_{0}$ was the maximal index for which the value $n_{i}^{\uparrow}(M)$ is attained for $M$, as we have seen). Therefore the maximal sum for $M^{\prime}$ is attained for the index $l_{0}+1$, and its value is $n_{i}^{\uparrow}\left(M^{\prime}\right)=\sum_{j \geq l_{0}+1}\left(M_{i+1, j}-M_{i, j}\right)=n_{i}^{\uparrow}(M)-1$; by induction $e_{i}^{\uparrow}$ can be applied precisely that many times to $M^{\prime}$, and so it can be applied $n_{i}^{\uparrow}(M)$ times to $M$ as claimed. The statements for $e_{j}^{\leftarrow}, f_{i}^{\downarrow}$, and $f_{j}$ follow from the statement we just proved by considering the (finite) matrices obtained from $M$ by turning it one, two, or three quarter turns. The statements in the final sentence of the proposition are clear if one realises that for instance $\sum_{j<l}\left(M_{i, j}-M_{i+1, j}\right)$ and $\sum_{j \geq l}\left(M_{i+1, j}-M_{i, j}\right)$ differ by $\operatorname{row}(M)_{i}-\operatorname{row}(M)_{i+1}$ independently of $l$, so that their maxima $n_{i}^{\downarrow}(M)$ and $n_{i}^{\uparrow}(M)$ are attained for the same (set of) values of $l$, and also differ by $\operatorname{row}(M)_{i}-\operatorname{row}(M)_{i+1}$.

With respect to the possibility of successive moves between a pair of adjacent rows or columns, we can make a distinction between pairs whose interchange is forbidden in $M$ but can be made possible after some other exchanges between those rows or columns, and pairs whose interchange will remain forbidden regardless of such exchanges. We 
have seen that when a move is followed by a move in the opposite direction, the latter undoes the effect of the former; it follows that if a given move can be made possible by first performing one or more moves between the same pair of rows or columns, then one may assume that all those moves are in the same direction. Moreover we have seen for instance that successive upward moves between two rows always occur from left to right; this implies that if a pair $\left(\begin{array}{l}0 \\ 1\end{array}\right)$ in column $l$ of rows $i, i+1$ is not interchangeable due to a failure of some instance of the second inequality in 1.3.1a (which only involves columns $j>l$ ), then this circumstance will not be altered by any preceding upward moves between the same rows, and the move will therefore remain forbidden. On the other hand if the second inequality in 1.3.1a is satisfied for all $l^{\prime}>l$, then the value of $\sum_{j \geq l}\left(M_{i+1, j}-M_{i, j}\right)$ is larger than the one obtained by replacing the bound $l$ by any $l^{\prime}>l$; it may still be less than that overall maximum $n_{i}^{\uparrow}(M)$, but that value can be lowered by successive upward moves between rows $i, i+1$, which must necessarily occur in columns $j<l$, until the pair $\left(\begin{array}{l}0 \\ 1\end{array}\right)$ considered becomes interchangeable.

We may therefore conclude that, in the sense of repeated moves between two adjacent rows, failure of an instance of the first inequality in 1.3.1a gives a temporary obstruction for a candidate upward move, while failure of an instance of the second inequality gives a permanent obstruction. For candidate downward moves the situation is reversed. The following alternative description may be more intuitive. If one represents each pair $\left(\begin{array}{l}0 \\ 1\end{array}\right)$ by "(", each pair $\left(\begin{array}{l}1 \\ 0\end{array}\right)$ by ")", and all remaining pairs by "-" (or any nonparenthesis symbol), then for all parentheses that match another one in the usual sense, the pairs in the corresponding columns are permanently blocked. The remaining unmatched parentheses have the structure ") $\cdots)(\cdots$ (" of a sequence of right parentheses followed by a sequence of left parentheses (either of which might be an empty sequence). An upward move between these rows is possible in the column corresponding to the leftmost unmatched "(" if it exists, and a downward move between these rows is possible in the column corresponding to the rightmost unmatched ")" if it exists. In either case the move replaces the parenthesis by an opposite one, and since it remains unmatched, we can continue with the same description for considering subsequent moves. In this view it is clear that all unmatched parentheses can be ultimately inverted, and that upward moves are forced to occur from left to right, and downward moves from right to left. For instance, in the $3 \times 13$ matrix given as an example after definition 1.3.1, the sequence of symbols for the two topmost rows is " $)((-())((-((-"$, and from this it is clear that one downward move is possible in column 0 , or at most 5 successive upward moves in columns $1,7,8,10$, and 11 ; for the bottommost rows we have the sequence "--) - - - (-- - - - " and only successive downward moves are possible, in columns 4 and 2. For moves between adjacent columns the whole picture described here must be rotated a quarter turn (clockwise or counterclockwise, this makes no difference).

We now consider the relation of the definitions above to the tableau conditions and Littlewood-Richardson conditions on matrices. The first observation is that these conditions can be stated in terms of the potentials for raising (or for lowering) operations.

1.3.5. Proposition. Let $M \in \mathcal{M}^{[2]}$ and let $\lambda / \kappa$ and $\mu / \nu$ be skew shapes.

(1) $M \in \operatorname{Tabl}^{[2]}(\lambda / \kappa)$ if and only if $\operatorname{col}(M)=\lambda^{t}-\kappa^{t}$ and $n_{j}^{\leftarrow}(M) \leq \kappa_{j}^{t}-\kappa_{j+1}^{t}$ for 


\subsection{Crystal operations for binary matrices}

all $j \in \mathbf{N}$.

(2) $M \in \operatorname{LR}^{[2]}(\nu / \mu)$ if and only if $\operatorname{row}(M)=\nu-\mu$ and $n_{i}^{\uparrow}(M) \leq \mu_{i}-\mu_{i+1}$ for all $i \in \mathbf{N}$. The second parts of these conditions can also be stated in terms of the potentials of $M$ for lowering operations, as $n_{j}(M) \leq \lambda_{j}^{t}-\lambda_{j+1}^{t}$ for all $j \in \mathbf{N}$, respectively as $n_{i}^{\downarrow}(M) \leq \nu_{i}-\nu_{i+1}$ for all $i \in \mathbf{N}$.

Proof. In view of the expressions in definition 1.3.3, these statements are just reformulations of the parts of proposition 1.1.1 and definition 1.1.2 that apply to binary matrices.

The next proposition shows that vertical and horizontal crystal operations on matrices respect the tableau conditions respectively the Littlewood-Richardson conditions for all skew shapes at once.

1.3.6. Proposition. If binary matrices $M, M^{\prime} \in \mathcal{M}^{[2]}$ are related by $M^{\prime}=e_{i}^{\uparrow}(M)$ for some $i \in \mathbf{N}$, then $n_{j}^{\leftarrow}(M)=n_{j}^{\leftarrow}\left(M^{\prime}\right)$ and $n_{j}(M)=n_{j}\left(M^{\prime}\right)$ for all $j \in \mathbf{N}$. Consequently, the conditions $M \in \operatorname{Tabl}^{[2]}(\lambda / \kappa)$ and $M^{\prime} \in \operatorname{Tabl}^{[2]}(\lambda / \kappa)$ are equivalent for any skew shape $\lambda / \kappa$. Similarly if $M$ and $M^{\prime}$ are related by $M^{\prime}=f_{j}(M)$ for some $j \in \mathbf{N}$, then $n_{i}^{\uparrow}(M)=n_{i}^{\uparrow}\left(M^{\prime}\right)$ and $n_{i}^{\downarrow}(M)=n_{i}^{\downarrow}\left(M^{\prime}\right)$ for all $i \in \mathbf{N}$, and $M \in \operatorname{LR}^{[2]}(\nu / \mu) \Longleftrightarrow M^{\prime} \in$ $\operatorname{LR}^{[2]}(\nu / \mu)$ for any skew shape $\nu / \mu$.

Proof. It suffices to prove the statements about $M^{\prime}=e_{i}^{\uparrow}(M)$, since those concerning $M^{\prime}=e_{j}^{\leftarrow}(M)$ will then follow by applying the former to matrices obtained by rotating $M$ and $M^{\prime}$ a quarter turn counterclockwise. For the case considered it will moreover suffice to prove $n_{j}^{\leftarrow}(M)=n_{j}^{\leftarrow}\left(M^{\prime}\right)$ for any $j \in \mathbf{N}$, since $n_{j}(M)=n_{\vec{j}}\left(M^{\prime}\right)$ will then follow from $\operatorname{col}(M)=\operatorname{col}\left(M^{\prime}\right)$, and the equivalence of $M \in \operatorname{Tabl}^{[2]}(\lambda / \kappa)$ and $M^{\prime} \in$ $\operatorname{Tabl}^{[2]}(\lambda / \kappa)$ will be a consequence of proposition 1.3.5. One may suppose that the pair of bits being interchanged to obtain $M^{\prime}$ from $M$ is in column $j$ or $j+1$, since otherwise $n_{j}^{\leftarrow}(M)=n_{j}^{\leftarrow}\left(M^{\prime}\right)$ is obvious from (13). Let $\left(p_{k}\right)_{k \in \mathbf{N}}$ be the sequence of partial sums for $M$ of which $n_{j}^{\leftarrow}(M)$ is the maximum, in other words $p_{k}=\sum_{i^{\prime}<k}\left(M_{i^{\prime}, j+1}-M_{i^{\prime}, j}\right)$, and let $\left(p_{k}^{\prime}\right)_{k \in \mathbf{N}}$ be the corresponding sequence for $M^{\prime}$. Then the only index $k$ for which $p_{k} \neq p_{k}^{\prime}$ is $k=i+1$ : one has $p_{i+1}^{\prime}=p_{i+1}-1$ if the move occurred in column $j$, or $p_{i+1}^{\prime}=p_{i+1}+1$ if it occurred in column $j+1$. The only way in which this change could make $n_{j}^{\leftarrow}(M)=\max _{k} p_{k}$ differ from $n_{j}^{\leftarrow}\left(M^{\prime}\right)=\max _{k} p_{k}^{\prime}$ is if $k=i+1$ were the unique index for which $p_{k}=n_{j}^{\leftarrow}(M)$ (in the former case) or for which $p_{k}^{\prime}=n_{j}^{\leftarrow}\left(M^{\prime}\right.$ ) (in the latter case). That would in particular require that the indicated value be strictly larger than $p_{k}=p_{k}^{\prime}$ and than $p_{k+2}=p_{k+2}^{\prime}$, so $M$ or $M^{\prime}$ would have to contain a submatrix $\left(\begin{array}{ll}0 & 1 \\ 1 & 0\end{array}\right)$ at the intersection of rows $i, i+1$ and columns $j, j+1$, while the other matrix would differ by the interchange of one of those two vertically adjacent pairs of bits. But we have seen that in such a submatrix neither of those two pairs of bits can be interchangeable, which excludes this possibility, and one therefore has $n_{j}^{\leftarrow}(M)=n_{j}^{\leftarrow}\left(M^{\prime}\right)$ in all cases.

One can summarise the last two propositions as follows: Littlewood-Richardson conditions can be stated in terms of the potentials for vertical moves, which moves preserve tableau conditions, while tableau conditions can be stated in terms of the potentials for horizontal moves, which moves preserve Littlewood-Richardson conditions. 
We shall now outline the way in which crystal operations can be used to define cancellations either of terms for matrices not in $\operatorname{Tabl}^{[2]}(\lambda / \kappa)$ or of those not in $\operatorname{LR}^{[2]}(\nu / \mu)$, in the summations of (1), (2), or (7). One starts by traversing each matrix $M$ as before, searching for a violation of the condition in question, and of an index that witnesses it; this amounts to finding a raising operation $e$ (i.e., some $e_{j}^{\leftarrow}$ or $e_{i}^{\uparrow}$ ) for which the potential of $M$ is larger than allowed by the second part of proposition 1.3.5 (1) or (2).

Now consider the set of matrices obtainable from $M$ by a sequence of applications of $e$ or of its inverse lowering operation $f$; these form a finite "ladder" in which the operation $e$ moves up, and $f$ moves down. Note that the potential for $e$ increases as one descends the ladder. The condition of having a too large a potential for $e$ determines a lower portion containing $M$ of the ladder, for which all corresponding terms must be cancelled, and the witness chosen for such a cancellation will be the same one as chosen for $M$ (there may also be terms cancelled in the remaining upper portion of the ladder, but their witnesses will be different). Now the modification of $\alpha$ needed to ensure the change of the sign of $\varepsilon(\alpha, \lambda)$ can be obtained by reversing that lower part of the ladder. Since a pair of matrices whose terms cancel are thus linked by a sequence of horizontal or vertical moves, their status for any Littlewood-Richardson condition respectively tableau condition (the kind for which one is not cancelling) will be the same, which allows this cancellation to be used as a second phase (starting from (7) or (2)).

Let us fill in the details of the description above, for the cancellation of terms for matrices not in $\operatorname{LR}^{[2]}(\nu / \mu)$, in other words leading from (1) to (7) of from (2) to (3). As described at the beginning of this subsection, we start by finding the maximal index $l$ such that the composition $\beta=\mu+\sum_{j \geq l} M_{j}^{t}$ is not a partition, and choosing an index $i$ for which $\beta_{i+1}=\beta_{i}+1$; this implies that $n_{i}^{\uparrow}(M)>\mu_{i}-\mu_{i+1}$, so the potential of $M$ for $e=e_{i}^{\uparrow}$ exceeds the limit given in 1.3.5 (2). For convenience let us use the notation $e^{d}$ to stand for $\left(e_{i}^{\uparrow}\right)^{d}$ when $d>0$, for the identity when $d=0$, and for $\left(f_{i}^{\downarrow}\right)^{-d}$ when $d<0$; then the ladder mentioned above is $\left\{e^{d}(M) \mid-n_{i}^{\downarrow}(M) \leq d \leq n_{i}^{\uparrow}(M)\right\}$, and its lower part whose elements must be cancelled because of a too large potential for $e_{i}^{\uparrow}$ is $\left\{e^{d}(M) \mid-n_{i}^{\downarrow}(M) \leq d<n_{i}^{\uparrow}(M)-\left(\mu_{i}-\mu_{i+1}\right)\right\}$.

From the maximality of $l$ it follows that $M$ contains a pair $\left(\begin{array}{l}0 \\ 1\end{array}\right)$ in column $l$ of rows $i, i+1$, and that this pair is not permanently blocked for upward moves in those rows (in other words, one has $\sum_{j=l+1}^{m} M_{i, j} \leq \sum_{j=l+1}^{m} M_{i+1, j}$ for all $m>l$ ); indeed the pair will be interchanged upon applying $e^{d}$ to $M$ whenever $n_{i}^{\uparrow}(M)-\left(\mu_{i}-\mu_{i+1}\right) \leq d \leq n_{i}^{\uparrow}(M)$, i.e., in the mentioned upper part of the ladder. So the lower part of the ladder is precisely the part in which that pair is not interchanged, and the matrices in this part will give rise to the same indices $l$ and $i$ as $M$ to witness their cancellation. The expression for $d$ such that the term for $M$ cancels against the one for $e^{d}(M)$ can be found as follows. If $M$ is at the bottom of the ladder $\left(n_{i}^{\downarrow}(M)=0\right)$ then $d$ has the value $n_{i}^{\uparrow}(M)-\left(\mu_{i}-\mu_{i+1}\right)-1$ that gives the topmost value of the bottom part of the ladder, and $d$ decreases with the level $n_{i}^{\downarrow}(M)$ of $M$, so the expression is $d=n_{i}^{\uparrow}(M)-\left(\mu_{i}-\mu_{i+1}\right)-1-n_{i}^{\downarrow}(M)$. Putting $\alpha=\mu+\operatorname{row}(M)$, this can also be written as $d=\alpha_{i+1}-\alpha_{i}-1$, by proposition 1.3.4. Since each application of $e_{i}^{\uparrow}$ increases the sum of entries in row $i$ while decreasing the sum in row $i+1$, the value $\alpha^{\prime}=\mu+\operatorname{row}\left(M^{\prime}\right)$ for the matrix $M^{\prime}=e^{d}(M)$ satisfies 


\subsection{Crystal operations for binary matrices}

$\left(\alpha_{i}^{\prime}, \alpha_{i+1}^{\prime}\right)=\left(\alpha_{i+1}-1, \alpha_{i}+1\right)$ while its remaining components are unchanged from $\alpha$, which ensures that $\varepsilon(\alpha, \nu)+\varepsilon\left(\alpha^{\prime}, \nu\right)=0$. The fact that $M$ and $M^{\prime}$ are related by vertical moves implies that $M \in \operatorname{Tabl}^{[2]}(\lambda / \kappa) \Longleftrightarrow M^{\prime} \in \operatorname{Tabl}^{[2]}(\lambda / \kappa)$ for any skew shape $\lambda / \kappa$, so the terms for $M$ and $M^{\prime}$ do indeed cancel, whether we are considering the passage from (1) to (7) or the one from (2) to (3).

For the cancellations involved in passing from (1) to (2) and from (7) to (3) the description is similar, but rotated a quarter turn counterclockwise: the initial scan of the matrix is by rows from top to bottom, and the raising operations $n_{i}^{\uparrow}$ are replaced by raising operations $n_{j}^{\leftarrow}$.

The reader may have been wondering whether we have been going through all these details just to obtain more aesthetically pleasing descriptions of the reductions $(1) \rightarrow(2) \rightarrow(3)$ and $(1) \rightarrow(7) \rightarrow(3)$ (and maybe the reader even doubts whether that goal was actually obtained). But crystal operations turn out to be useful in other ways than just to define cancellations, and several such applications will be given below; those applications alone largely justify the definition of crystal operations. We have nevertheless chosen to introduce them by considering cancellations, because that provides a motivation for the precise form of their definition and for treating separate cases for binary and integral matrices; such motivation might otherwise not be evident. For our further applications it is of crucial importance that horizontal and vertical moves are compatible in a stronger sense than expressed in proposition 1.3.6. Not only do moves in one direction leave invariant the potentials for all moves in perpendicular directions, they actually commute with those moves, as stated in the following lemma.

1.3.7. Lemma. (binary commutation lemma) Let $M, M^{\prime}, M^{\prime \prime} \in \mathcal{M}^{[2]}$ be related by $M^{\prime}=e_{i}^{\uparrow}(M)$ and $M^{\prime \prime}=e_{j}^{\leftarrow}(M)$ for some $i, j \in \mathbf{N}$; then $e_{j}^{\leftarrow}\left(M^{\prime}\right)=e_{i}^{\uparrow}\left(M^{\prime \prime}\right)$. The same holds when $e_{i}^{\uparrow}$ is replaced both times by $f_{i}^{\downarrow}$ and/or $e_{j}^{\leftarrow}$ is replaced both times by $f_{j}$.

Proof. Note that the expressions $e_{j}^{\leftarrow}\left(M^{\prime}\right)$ and $e_{i}^{\uparrow}\left(M^{\prime \prime}\right)$ are defined since $n_{j}^{\leftarrow}\left(M^{\prime}\right)=$ $n_{j}^{\leftarrow}(M)>0$ and $n_{i}^{\uparrow}\left(M^{\prime \prime}\right)=n_{i}^{\uparrow}(M)>0$ by proposition 1.3.6. The variants given in the second part of the lemma can be deduced from the initial statement either by rotation symmetry or by a suitable change of roles between the four matrices involved. So we shall focus on proving the initial statement.

Suppose first that the pairs of bits interchanged in the moves $e_{i}^{\uparrow}: M \mapsto M^{\prime}$ and $e_{j}^{\leftarrow}: M \mapsto M^{\prime \prime}$ are disjoint. In this case we shall argue that these pairs of bits are in the same position as the pairs of bits interchanged in the moves $e_{i}^{\uparrow}: M^{\prime \prime} \mapsto e_{i}^{\uparrow}\left(M^{\prime \prime}\right)$ and $e_{j}^{\leftarrow}: M^{\prime} \mapsto e_{j}^{\leftarrow}\left(M^{\prime}\right)$, respectively; then it will be obvious that $e_{j}^{\leftarrow}\left(e_{i}^{\uparrow}(M)\right)=e_{i}^{\uparrow}\left(e_{j}^{\leftarrow}(M)\right)$. To this end must show that the conditions in definition 1.3.1, which are satisfied in $M$ for each of the two pairs of bits considered, remain valid after the other pair is interchanged. Since the values of one pair of bits is not affected by the interchange of the other pair, we only need to worry about the four inequalities in that definition. Depending on the relative positions of the two pairs, at most one of those inequalities can have an instance for which the values being compared change, but since we do not know which one, this does not help us much; nevertheless the four cases are quite similar, so we shall treat only the first one explictly. Each inequality, with its quantification, can be reformulated as stating that some maximum of partial sums does not exceed 0 (actually it equals 0 ); for 
instance the first inequality is equivalent to ' $\max \left\{\sum_{j=l^{\prime}}^{l-1}\left(M_{k+1, j}-M_{k, j}\right) \mid 0 \leq l^{\prime} \leq l\right\} \leq$ 0 ' (this condition applies for $k=i$ if the move of $e_{i}^{\uparrow}$ occurs in column $l$ ). That maximum of partial sums is of the same type as the one in one of the equations (11)-(14), but for a truncated matrix; in the cited case they are the partial sums of (11) but computed for $M$ truncated to its columns $j<l$. Therefore the same reasoning as in the proof of proposition 1.3.6 shows that although one of the partial sums may change, their maximum remains the same, so that the pair of bits considered remains interchangeable.

Now suppose that to the contrary the pairs of bits $\left(\begin{array}{l}0 \\ 1\end{array}\right)$ and $\left(\begin{array}{ll}0 & 1\end{array}\right)$ being interchanged in $M$ do overlap. Then after performing one interchange, the pair of bits in the position of the other pair can no longer be interchangeable, as its bits will have become equal. There is a unique $2 \times 2$ submatrix of $M$ that contains the two overlapping pairs, and since it contains both a vertical and a horizontal interchangeable pair of bits, its value can be neither $\left(\begin{array}{ll}0 & 1 \\ 1 & 0\end{array}\right)$ nor $\left(\begin{array}{ll}1 & 0 \\ 0 & 1\end{array}\right)$. Therefore it will contain either $\left(\begin{array}{ll}0 & 1 \\ 1 & 1\end{array}\right)$ if the two pairs overlap in their bit ' 0 ' (at the top left), or $\left(\begin{array}{ll}0 & 0 \\ 0 & 1\end{array}\right)$ if the two pairs overlap in their bit ' 1 ' (at the bottom right). In either case it is not hard to see that the overlapping bit, after having been interchanged horizontally or vertically, is again (in its new position) part of an interchangeable pair within the $2 \times 2$ submatrix, in the direction perpendicular to the move performed; the other bit of that pair is the one in the corner diametrically opposite to the old position of the overlapping bit in the submatrix considered (the bottom right corner in the former case and the top left corner in the latter case). This is so because comparing that new pair with the interchangeable pair that used to be in the remaining two squares of the $2 \times 2$ submatrix, the only difference for each of the pertinent inequalities of definition 1.3.1 is the insertion or removal of a bit with the same value in each of the two sums being compared, which does not affect the result of the comparison. Therefore the succession of two raising operations, applied in either order, will transform the submatrix $\left(\begin{array}{ll}0 & 1 \\ 1 & 1\end{array}\right)$ into $\left(\begin{array}{ll}1 & 1 \\ 1 & 0\end{array}\right)$, or the submatrix $\left(\begin{array}{ll}0 & 0 \\ 0 & 1\end{array}\right)$ into $\left(\begin{array}{ll}1 & 0 \\ 0 & 0\end{array}\right)$, as illustrated below.

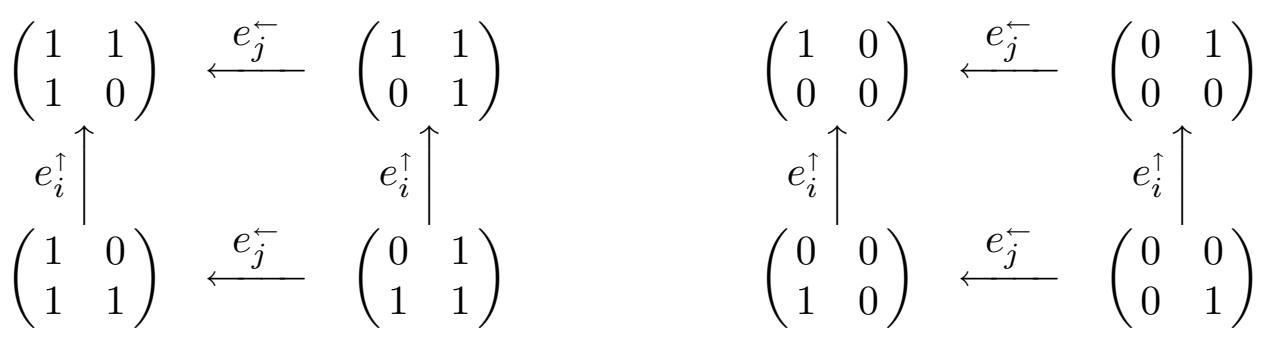

\subsection{Crystal operations for integral matrices.}

Motivated by the existence of cancellations $(4) \rightarrow(5) \rightarrow(6)$ and $(4) \rightarrow(8) \rightarrow(6)$, we shall now define operations like those defined in the previous subsection, but for integral instead of binary matrices. Much of what will be done in this subsection is similar to what was done before, so we shall focus mainly on the differences with the situation for binary matrices.

A first difference is the fact that for integral matrices the operation of interchanging adjacent entries is too restrictive to achieve the desired kind of modifications. We shall therefore regard each matrix entry $m$ as if it were a pile of $m$ units, and the basic type 


\subsection{Crystal operations for integral matrices}

of operation will consist of moving some units from some entry $m>0$ to a neighbouring entry, which amounts to decreasing the entry $m$ and increasing the neighbouring entry by the same amount. We shall call this a transfer between the two entries; as in the binary case we shall impose conditions for such a transfer to be allowed. Another difference is the kind of symmetry implicitly present in equation (6) compared with (3), which in fact stems from the difference between the cases of binary and integral matrices in the relation of definition 1.1.2 to proposition 1.1.1, which we already observed following that definition. As a result, the rules for allowing transfers will not be symmetric with respect to rotation by a quarter turn, but instead they will be symmetric with respect to transposition of the integral matrices and with respect to rotation by a half turn.

This new type of symmetry somewhat simplifies the situation, but there is also a complicating factor, due to the fact that the tableau conditions and LittlewoodRichardson conditions are more involved for integral matrices than for binary ones. In the binary case it sufficed to construct a sequence of compositions by cumulating rows or columns, and to test each one individually for being a partition. But in the integral case one must test for each pair $\alpha, \beta$ of successive terms in the sequence whether $\alpha-\beta$, in other words whether $\beta / \alpha$ is a horizontal strip. That test amounts to verifying $\beta_{i+1} \leq \alpha_{i}$ for all $i$, since $\alpha_{i} \leq \beta_{i}$ already follows from the circumstance that $\beta_{i}$ is obtained by adding a matrix entry to $\alpha_{i}$. Thus if we focus on the inequalities involving the parts $i$ and $i+1$ of the compositions in the sequence, then instead of just checking that part $i+1$ never exceeds part $i$ of the same composition, one must test the stronger requirement that part $i+1$ of the next partition in the sequence still does not exceed that (old) part $i$.

This will mean for the analogues of definitions 1.3.1 and 1.3.3, that the final entries in partial sums in two adjacent rows or columns will not be in the same column or row, but in a diagonal position with respect to each another (always in the direction of the main diagonal). This also means that the conditions required to allow a transfer must take into account some of the units that are present in the matrix entries between which the transfer takes place, but which are not being transferred themselves (in the binary case no such units exist). Although the precise form of the following definition could be more or less deduced from the properties we seek, we shall just state it, and observe afterwards that it works.

1.4.1. Definition. Let $M \in \mathcal{M}, k, l \in \mathbf{N}$, and $a \in \mathbf{Z}-\{0\}$.

a. Suppose that $\sum_{j=l^{\prime}}^{l-1}\left(M_{k+1, j+1}-M_{k, j}\right) \geq \max (a, 0)$ for all $l^{\prime}<l$, or if $l=0$ that $M_{k+1,0} \geq a$, and suppose $\sum_{j=l}^{l^{\prime}-1}\left(M_{k, j}-M_{k+1, j+1}\right) \geq \max (-a, 0)$ for all $l^{\prime}>l$. Then we allow the entries $\left(M_{k, l}, M_{k+1, l}\right)$ to be replaced by $\left(M_{k, l}+a, M_{k+1, l}-a\right)$; this is called an upward transfer of a units between rows $k$ and $k+1$ if $a>0$, or a downward transfer of $-a$ units between those rows if $a<0$.

b. Suppose that $\sum_{i=k^{\prime}}^{k-1}\left(M_{i+1, l+1}-M_{i, l}\right) \geq \max (a, 0)$ for all $k^{\prime}<k$, or if $k=0$ that $M_{0, l+1} \geq a$, and suppose $\sum_{i=k}^{k^{\prime}-1}\left(M_{i, l}-M_{i+1, l+1}\right) \geq \max (-a, 0)$ for all $k^{\prime}>k$. Then we allow the entries $\left(M_{k, l}, M_{k, l+1}\right)$ to be replaced by $\left(M_{k, l}+a, M_{k, l+1}-a\right)$; this is called a leftward transfer of a units between columns $l$ and $l+1$ if $a>0$, or a rightward transfer of $-a$ units between those columns if $a<0$. 
Remarks. (1) The occurrence of the quantity $a$ in the inequalities has the effect of cancelling its contribution to the entry from which it would be transferred. It follows that the transfer can always be followed by a transfer of $a$ units in the opposite sense between the same entries, which reconstructs the original matrix. (2) The exceptional conditions $M_{0, l+1} \geq a$ and $M_{k+1,0} \geq a$ compensate for the absence of any inequality where $a$ occurs in the way just mentioned. They serve to exclude the introduction of negative entries by a transfer; note that for instance this is taken care of for upward moves with $l>0$ by the condition $M_{k+1, l}-M_{k, l-1} \geq a$, and for downward moves by $M_{k, l}-M_{k+1, l+1} \geq-a$. Hence the cases $k=0$ and $l=0$ are not treated any differently from the others. (3) We could have restricted the definition to the cases $a=1$ and $a=$ -1 , since transfers with $|a|>1$ can be realised by repeated transfers with $|a|=1$. The current formulation was chosen for readability, and because it permits a straightforward and interesting generalisation to matrices with non-negative real coefficients.

We shall call these transfers crystal operations on integral matrices. It can be seen that horizontal transfers correspond to $|a|$ successive coplactic operations on the word formed by concatenating weakly decreasing words whose weights are given by the (nonzero) rows of $M$, taken from top to bottom; vertical transfers correspond $|a|$ successive to coplactic operations on the word similarly formed by concatenating weakly decreasing words with weights given by the columns of $M$, taken from left to right. Horizontal transfers in the integral encoding of a semistandard tableau $T$ correspond to coplactic operations on $T$.

Here is a small example of vertical transfers; for an example of horizontal transfers one can transpose the matrix. Consider vertical moves between the two nonzero rows of the integral matrix

$$
M=\left(\begin{array}{lllllllll}
1 & 2 & 1 & 3 & 3 & 1 & 2 & 4 & 0 \\
2 & 1 & 1 & 4 & 2 & 0 & 5 & 2 & 0
\end{array}\right)
$$

For the three leftmost columns no transfer is possible because $\sum_{j=l}^{2}\left(M_{0, j}-M_{1, j+1}\right)<0$ for $l \in\{0,1,2\}$. For column 3 however an upward transfer of at most 2 units is possible, where the limit comes from the value $\sum_{j=1}^{2}\left(M_{1, j+1}-M_{0, j}\right)=2$ (and starting the sum at $j=0$ would give the same value). No downward transfer is possible in that column because $\sum_{j=3}^{5}\left(M_{0, j}-M_{1, j+1}\right)=0$; however a downward move of at most 4 units is possible in column 7 . If all 4 units are transferred downwards in that column it is replaced by $\left(\begin{array}{l}0 \\ 6\end{array}\right)$, and no further downwards between these rows will be possible; however if 2 units are transferred upwards in column 3 one obtains the matrix

$$
M^{\prime}=\left(\begin{array}{lllllllll}
1 & 2 & 1 & 5 & 3 & 1 & 2 & 4 & 0 \\
2 & 1 & 1 & 2 & 2 & 0 & 5 & 2 & 0
\end{array}\right)
$$

and an upwards transfer in $M^{\prime}$ is still possible, namely in column 0 where both units from row 1 can be transferred to row 0 .

We now consider the ordering of transfers between a given pair of adjacent rows or columns. Since any simultaneous transfer of more than one unit can be broken up 


\subsection{Crystal operations for integral matrices}

into smaller transfers, we cannot claim that at most one transfer in a given direction between a given pair of rows or columns is possible, but it is important that the only choice concerns the amount transferred, not the pair of entries where the transfer takes places. Now if vertical transfers of $a$ and $a^{\prime}$ units are possible between rows $i$ and $i+1$, respectively in columns $j_{0}$ and $j_{1}$ with $j_{0}<j_{1}$, then the inequalities in 1.4.1a give the inequalities $\min (a, 0) \geq \sum_{j=j_{0}}^{j_{1}-1}\left(M_{j+1, i+1}-M_{j, i}\right) \geq \max \left(a^{\prime}, 0\right)$ of which the middle member must be 0 , and which together with $a, a^{\prime} \neq 0$ therefore imply $a>0>a^{\prime}$. This shows that the transfer in column $j_{0}$ is upwards and the one in column $j_{1}$ is downwards. Thus successive upward transfers between the same pair of rows take place in columns whose index decreases weakly, and successive downward transfers between these rows take place in in columns whose index increases weakly; the same is true with the words "rows" and "columns" interchanged. Note that these directions are opposite to the ones in the binary case for transfers between rows, but they are the same as the ones in the binary case for transfers between columns. The following definition is now justified.

1.4.2. Definition. (integral raising and lowering operations) Let $M \in \mathcal{M}$ and $i, j \in \mathbf{N}$.

a. If $M$ admits an upward or downward transfer of a single unit between rows $i$ and $i+$ 1 , then the resulting matrix is denoted by $e_{i}^{\uparrow}(M)$ respectively by $f_{i}^{\downarrow}(M)$. If $M$ admits no upward or no downward transfer between rows $i$ and $i+1$, then the expression $e_{i}^{\uparrow}(M)$ respectively $f_{i}^{\downarrow}(M)$ is undefined.

b. If $M$ admits a leftward or rightward transfer of a single unit between columns $j$ and $j+1$, then the resulting matrix is denoted by $e_{j}^{\leftarrow}(M)$ respectively by $f_{j}(M)$. If $M$ admits no leftward or no rightward transfer between columns $j$ and $j+1$, then the expression $e_{j}^{\leftarrow}(M)$ respectively $f_{j}(M)$ is undefined.

If an upward, downward, leftward, or rightward transfer of $a>1$ units between a given pair of rows $i, i+1$ or columns $j, j+1$ is defined, the the resulting matrix can also be obtained by a succession of $a$ raising or lowering operations, as $\left(e_{i}^{\uparrow}\right)^{a}(M),\left(f_{i}^{\downarrow}\right)^{a}(M)$, $\left(e_{j}^{\leftarrow}\right)^{a}(M)$ or $\left(f_{j}\right)^{a}(M)$, respectively. A succession of even more transfers between the same rows or columns and in the same direction may be possible, and the potentials for such transfers are given by the following expressions.

1.4.3. Definition. For $M \in \mathcal{M}$ and $i, j \in \mathbf{N}$, the numbers $n_{i}^{\uparrow}(M), n_{i}^{\downarrow}(M), n_{j}^{\leftarrow}(M)$, $n_{j}(M) \in \mathbf{N}$ are defined by

$$
\begin{aligned}
n_{i}^{\uparrow}(M) & =\max \left\{M_{i+1,0}+\sum_{j<l}\left(M_{i+1, j+1}-M_{i, j}\right) \mid l \in \mathbf{N}\right\}, \\
n_{i}^{\downarrow}(M) & =\max \left\{\sum_{j \geq l}\left(M_{i, j}-M_{i+1, j+1}\right) \mid l \in \mathbf{N}\right\}, \\
n_{j}^{\leftarrow}(M) & =\max \left\{M_{0, j+1}+\sum_{i<k}\left(M_{i+1, j+1}-M_{i, j}\right) \mid k \in \mathbf{N}\right\}, \\
n_{j}(M) & =\max \left\{\sum_{i \geq k}\left(M_{i, j}-M_{i+1, j+1}\right) \mid k \in \mathbf{N}\right\} .
\end{aligned}
$$

1.4.4. Proposition. For $M \in \mathcal{M}$ and $i, j \in \mathbf{N}$, the numbers of times each of $e_{i}^{\uparrow}, f_{i}^{\downarrow}$, , $e_{j}^{\leftarrow}$ and $f_{j}$ can be successively applied to $M$ are given respectively by the numbers $n_{i}^{\uparrow}(M), n_{i}^{\downarrow}(M), n_{j}^{\leftarrow}(M)$, and $n_{j}(M)$. Moreover $n_{i}^{\downarrow}(M)-n_{i}^{\uparrow}(M)=\operatorname{row}(M)_{i}-\operatorname{row}(M)_{i+1}$ and $n_{j}(M)-n_{j}^{\leftarrow}(M)=\operatorname{col}(M)_{j}-\operatorname{col}(M)_{j+1}$.

Proof. If $M$ admits an upward transfer of $a$ units between rows $i$ and $i+1$ and in column $l$, then $n_{i}^{\uparrow}(M) \geq M_{i+1,0}+\sum_{j<l}\left(M_{i+1, j+1}-M_{i, j}\right) \geq M_{i+1,0}+a>0$ if $l>0$, 
while one has $n_{i}^{\uparrow}(M) \geq M_{i+1,0} \geq a>0$ in case $l=0$, so $n_{i}^{\uparrow}(M)$ is nonzero either way. Similarly if $M$ admits a downward transfer of $a$ units between those rows then $n_{i}^{\downarrow}(M) \geq a>0$. Now suppose conversely that $n_{i}^{\uparrow}(M)>0$ or that $n_{i}^{\downarrow}(M)>0$. In the former case let $l_{0}$ be the minimal index $l$ for which the maximum in (18) is attained, and in the latter case let $l_{1}$ be the maximal index $l$ for which the maximum in (19) is attained; in either case let the maximum exceed all values at smaller respectively at larger indices by $a$. Then it is easily verified that an upward transfer of $a$ units in column $l_{0}$, respectively a downward transfer of $a$ units in column $l_{1}$, is possible between rows $i, i+1$. We know that this is the only column in which a transfer in that direction between rows $i, i+1$ is possible. Moreover the expressions in (18) and (19) have a constant difference row $(M)_{i+1}-\operatorname{row}(M)_{i}$ for every $l$, so we may conclude that, whenever a transfer in either direction between $M_{i, l}$ and $M_{i+1, l}$ is possible, the index $l$ realises the maxima defining $n_{i}^{\uparrow}(M)$ and $n_{i}^{\downarrow}(M)$ in both expressions. Then the fact that the transfer can be followed by an inverse transfer shows that an upward transfer of $a$ units decreases $n_{i}^{\uparrow}(M)$ by $a$, and that a downward transfer of $a$ units decreases $n_{i}^{\downarrow}(M)$ by $a$; a straightforward induction on $n_{i}^{\uparrow}(M)$ or $n_{i}^{\downarrow}(M)$ will complete the proof. The statements concerning $n_{j}^{\leftarrow}(M)$ and $n_{j}(M)$ follow by transposition of the matrices involved.

Like for binary case, we can describe the possibilities for transfers between a given pair of rows or columns using parentheses. We shall describe the case of rows $i, i+1$, where the notion of matching parentheses is most easily visualised; for the case of adjacent columns one must apply transposition of the matrix (unlike the binary case, where rotation must be used). Since successive transfers between two rows progress in the sense opposite to the binary case, namely from right to left for successive upward moves, we must invert the correspondence between parentheses and moves: an upward transfer of a unit will correspond to the transformation of ")" into "(". Each unit in row $i$ is a potential candidate for an downward transfer, and therefore represented by "(", and each unit in row $i+1$ is represented by ")". All these symbols are gathered basically from left to right to form a string of parentheses, but the crucial point is how to order the symbols coming from a same column $j$. The form of the summations in the definitions above makes clear that the rule must be to place the $M_{i, j}$ symbols "("to the right of the $M_{i+1, j}$ symbols ")", so that these cannot match each other; rather the symbols from $M_{i, j}$ may match those from $M_{i+1, j+1}$. Now, as for the binary case, the units that may be transferred correspond to the unmatched parentheses, and the order in which they are transferred is such that the corresponding parentheses remain unmatched: upward moves transform unmatched symbols ")" into "(" from right to left, and downward moves transform unmatched symbols "(" into ")" from left to right. In the example given the string of parentheses is $))(\mid)((\mid)(\mid))))(((\mid))(((\mid)(\mid)))))((\mid))(((($, where we have inserted bars to separate the contributions from different columns, and underlined the maximal substrings with balanced parentheses; this makes clear that 4 successive upward transfers are possible in columns 3,3,0,0, or 4 successive downward transfers, all in (the final nonzero) column 7 .

Note that any common number of units present in entries $M_{i, j}$ and $M_{i+1, j+1}$ will correspond to matching parentheses both for transfers between rows $i, i+1$ and between columns $j, j+1$. Therefore no transfer between those rows or those columns will 


\subsection{Crystal operations for integral matrices}

alter the value $\min \left(M_{i, j}, M_{i+1, j+1}\right)$ (but it can be altered by transfers in other pairs of rows or columns). In fact one may check that those instances of the inequalities in definition 1.4.1 whose summation is reduced to a single term forbid any such transfer involving $M_{i, j}$ or $M_{i+1, j+1}$ when that entry is strictly less than the other, and in case it is initially greater or equal than the other they forbid transfers that would make it become strictly less.

The relation of the above definitions to tableau conditions and LittlewoodRichardson conditions for integral matrices is given by the following proposition, which like the one for the binary case is a direct translation of the pertinent parts of proposition 1.1.1 and definition 1.1.2.

1.4.5. Proposition. Let $M \in \mathcal{M}$ and let $\lambda / \kappa$ and $\mu / \nu$ be skew shapes.

(1) $M \in \operatorname{Tabl}(\lambda / \kappa)$ if and only if $\operatorname{row}(M)=\lambda-\kappa$ and $n_{i}^{\uparrow}(M) \leq \kappa_{i}-\kappa_{i+1}$ for all $i \in \mathbf{N}$.

(2) $M \in \operatorname{LR}(\nu / \mu)$ if and only if $\operatorname{col}(M)=\nu-\mu$ and $n_{j}^{\leftarrow}(M) \leq \mu_{j}-\mu_{j+1}$ for all $j \in \mathbf{N}$.

The second parts of these conditions can also be stated in terms of the potentials of $M$ for lowering operations, as $n_{i}^{\downarrow}(M) \leq \lambda_{i}-\lambda_{i+1}$ for all $i \in \mathbf{N}$, respectively as $n_{j}(M) \leq \nu_{j}-\nu_{j+1}$ for all $j \in \mathbf{N}$.

And as is the binary case, the potentials for transfers in a perpendicular direction are unchanged.

1.4.6. Proposition. If integral matrices $M, M^{\prime} \in \mathcal{M}$ are related by $M^{\prime}=e_{i}^{\uparrow}(M)$ for some $i \in \mathbf{N}$, then $n_{j}^{\leftarrow}(M)=n_{j}^{\leftarrow}\left(M^{\prime}\right)$ and $n_{j}(M)=n_{j}\left(M^{\prime}\right)$ for all $j \in \mathbf{N}$. Consequently, the conditions $M \in \operatorname{LR}(\nu / \mu)$ and $M^{\prime} \in \operatorname{LR}(\nu / \mu)$ are equivalent for any skew shape $\nu / \mu$. Similarly if $M$ and $M^{\prime}$ are related by $M^{\prime}=f_{j}(M)$ for some $j \in \mathbf{N}$, then $n_{i}^{\uparrow}(M)=$ $n_{i}^{\uparrow}\left(M^{\prime}\right)$ and $n_{i}^{\downarrow}(M)=n_{i}^{\downarrow}\left(M^{\prime}\right)$ for all $i \in \mathbf{N}$, and one has $M \in \operatorname{Tabl}(\lambda / \kappa) \Longleftrightarrow M^{\prime} \in$ $\operatorname{Tabl}(\lambda / \kappa)$ for any skew shape $\lambda / \kappa$.

Proof. Like for the binary case we may focus on proving $n_{j}^{\leftarrow}(M)=n_{j}^{\leftarrow}\left(M^{\prime}\right)$ when $M^{\prime}=e_{i}^{\uparrow}(M)$, and we may assume that the upward transfer involved in passing from $M$ to $M^{\prime}$ occurs in column $j$ or $j+1$. Suppose first that it occurs in column $j$. Then the only partial sum in (20) that differs between $M$ and $M^{\prime}$ is the one for $k=i+1$, which has $M_{i+1, j+1}-M_{i, j}$ as final term; it will decrease by 1 in $M^{\prime}$. But this decrease will not affect the maximum taken in that equation unless it was strictly greater than the previous partial sum, for $k=i$, which means that $M_{i, j}<M_{i+1, j+1}$; however that would contradict the supposition that $e_{i}^{\uparrow}$ involves $M_{i, j}$, so it does not happen. Suppose next that the upward transfer occurs in column $j+1$. Then the only one of the values of which the maximum is taken in (20) that differs between $M$ and $M^{\prime}$ is the one for $k=i$ : it is $M_{i, j+1}$ if $i=0$, and otherwise contains a partial sum with final term $M_{i, j+1}-M_{i-1, j}$; it will increase by 1 in $M^{\prime}$ in either case. But that increase will not affect the maximum unless the value for $k=i$ is at least as great as the one for $k=i+1$, which means $M_{i, j} \geq M_{i+1, j+1}$, but this would contradict the supposition that $e_{i}^{\uparrow}$ decreases $M_{i+1, j+1}$, so this does not happen either. Therefore $n_{j}^{\leftarrow}(M)=n_{j}^{\leftarrow}\left(M^{\prime}\right)$ holds in all cases.

Using crystal operations for integral matrices, it is possible to describe cancellations that will realise the transitions $(4) \rightarrow(5) \rightarrow(6)$ and $(4) \rightarrow(8) \rightarrow(6)$, as we have done for binary matrices. We shall describe the cancellation of the terms not satisfying 
$M \in \operatorname{LR}(\nu / \mu)$, which realises $(5) \rightarrow(6)$ and $(4) \rightarrow(8)$; the cancellation of the terms not satisfying $M \in \operatorname{Tabl}(\kappa / \lambda)$ is similar, transposing all operations. One starts traversing $M$ by rows from top to bottom, searching for the first index $k$ (if any) for which one has $\left(\mu+\sum_{i<k} M_{i}\right) \forall\left(\mu+\sum_{i \leq k} M_{i}\right)$, and then choosing the maximal witness $j$ for this, i.e., with $\left(\mu+\sum_{i<k} M_{i}\right)_{j}<\left(\mu+\sum_{i \leq k} M_{i}\right)_{j+1}$. This implies that $n_{j}^{\leftarrow}(M)>\mu_{j}-\mu_{j+1}$, and similarly to the binary case the cancellation is along the "ladder" of matrices obtainable from $M$ by operations $e_{j}^{\leftarrow}$ and $f_{j}$, reversing its lower portion where the potential for $e_{j}^{\leftarrow}$ exceeds $\mu_{j}-\mu_{j+1}$ : the term for $M$ is cancelled against the term for $M^{\prime}=\left(e_{j}^{\leftarrow}\right)^{d}(M)$, where $d=n_{j}^{\leftarrow}(M)-\left(\mu_{j}-\mu_{j+1}+1\right)-n_{j}(M)$, and as before $\left(e_{j}^{\leftarrow}\right)^{d}$ means $\left(f_{j}\right)^{-d}$ if $d<0$.

One can also write $d=\alpha_{j+1}-\alpha_{j}-1$ where $\alpha=\mu+\operatorname{col}(M)$, from which it easily follows that the terms for $M$ and $M^{\prime}$ do indeed cancel each other. The fact that the same values $k$ and $j$ will be found for $M^{\prime}$ (so that we have actually defined an involution on the set of cancelling terms) is most easily seen as follows, using the parenthesis description given above. Throughout the lower portion of the ladder, the unmatched parentheses ")" from the left, up to and including the one corresponding to the unit of $M_{k, j+1}$ that "causes" the inequality $\left(\mu+\sum_{i<k} M_{i}\right)_{j}<\left(\mu+\sum_{i \leq k} M_{i}\right)_{j+1}$, are unchanged, so $M_{i}^{\prime}=M_{i}$ for all $i<k$, and the entry $M_{k, j+1}^{\prime}$ remains large enough that the mentioned inequality still holds for $M^{\prime}$. Since no coefficients have changed that could cause any index larger than $j$ to become a witness for $\left(\mu+\sum_{i<k} M_{i}^{\prime}\right)+\left(\mu+\sum_{i \leq k} M_{i}^{\prime}\right)$, the index $j$ will still be the maximal witness of that relation for $M^{\prime}$. (The change to the entry $M_{k, j}$ could cause the index $j-1$ to become, or cease to be, another witness of this relation for $M^{\prime}$, so it is important here that $j$ be chosen as a maximal witness, unlike in the binary case where any systematic choice works equally well.)

Again it will be important in applications to strengthen proposition 1.4.6, in a way that is entirely analogous to the binary case. The proof in the integral case will be slightly more complicated however.

1.4.7. Lemma. (integral commutation lemma) Let $M, M^{\prime}, M^{\prime \prime} \in \mathcal{M}$, be related by $M^{\prime}=e_{i}^{\uparrow}(M)$ and $M^{\prime \prime}=e_{j}^{\leftarrow}(M)$ for some $i, j \in \mathbf{N}$; then $e_{j}^{\leftarrow}\left(M^{\prime}\right)=e_{i}^{\uparrow}\left(M^{\prime \prime}\right)$. The same holds when $e_{i}^{\uparrow}$ is replaced both times by $f_{i}^{\downarrow}$ and/or $e_{j}^{\leftarrow}$ is replaced both times by $f_{j}$.

Proof. As in the binary case, it suffices to prove the initial statement, and both members of the equation $e_{j}^{\leftarrow}\left(M^{\prime}\right)=e_{i}^{\uparrow}\left(M^{\prime \prime}\right)$ are well defined by proposition 1.4.6. That equation obviously holds in those cases where the applications of $e_{j}^{\leftarrow}$ and $e_{i}^{\uparrow}$ occurring in it are realised by unit transfers between the same pairs of entries as in the application of these operations to $M$. This will first of all be the case when the pairs of entries involved in the transfers $e_{i}^{\uparrow}: M \mapsto M^{\prime}$ and $e_{j}^{\leftarrow}: M \mapsto M^{\prime \prime}$ are disjoint: in that case, for each inequality in definition 1.4.1 required for allowing one transfer, an argument similar to the one given in the proof of proposition 1.4.6 shows that the minimum over all values $l^{\prime}$ or $k^{\prime}$ of its left hand member is unaffected by the other transfer.

In the remaining case where the pairs of entries involved in the two transfers overlap, they lie inside the $2 \times 2$ square at the intersection of rows $i, i+1$ and columns $j, j+1$. Since we are considering upward and leftward transfers, there are only two possibilities: if $M_{i, j} \geq M_{i+1, j+1}$, then both transfers are towards $M_{i, j}$, while if $M_{i, j}<M_{i+1, j+1}$ 


\subsection{Crystal operations for integral matrices}

then both transfers are from $M_{i+1, j+1}$. In either case there is for each transfer only one of the inequalities in definition 1.4.1 whose left hand member is affected by the other transfer, namely the one involving just $M_{i, j}$ and $M_{i+1, j+1}$. In case both transfers are towards $M_{i, j}$, the inequalities in question both read $M_{i, j}-M_{i+1, j+1} \geq \max (-1,0)=0$, and they continue to hold when $M_{i, j}$ is increased in $M^{\prime}$ and in $M^{\prime \prime}$. In case both transfers are from $M_{i+1, j+1}$, the inequalities both read $M_{i+1, j+1}-M_{i, j} \geq \max (1,0)=1$, and here the other transfer may invalidate the inequality: indeed the inequality will continue to hold, when $M_{i+1, j+1}$ is decreased by 1 in $M^{\prime}$ and in $M^{\prime \prime}$, if and only if $M_{i+1, j+1} \geq M_{i, j}+2$. Therefore the only case where the unit transfers involved in $e_{j}^{\leftarrow}\left(M^{\prime}\right)$ and in $e_{i}^{\uparrow}\left(M^{\prime \prime}\right)$ are not in the same squares as in $e_{j}^{\leftarrow}(M)$ and in $e_{i}^{\uparrow}(M)$, respectively, is when $M_{i+1, j+1}=M_{i, j}+1$. In that case an elementary verification, comparing the required inequalities in $M^{\prime}$ and $M^{\prime \prime}$ to those that are known to hold in $M$, shows that those transfers are from $M_{i, j+1}^{\prime}$ to $M_{i, j}^{\prime}$ respectively from $M_{i+1, j}^{\prime \prime}$ to $M_{i, j}^{\prime \prime}$; then $e_{j}^{\leftarrow}\left(M^{\prime}\right)=e_{i}^{\uparrow}\left(M^{\prime \prime}\right)$ still holds, as illustrated by the display below of the relevant $2 \times 2$ squares. Instead of that verification, one may also argue that the transfer involved in $e_{j}^{\leftarrow}\left(M^{\prime}\right)$ or in $e_{i}^{\uparrow}\left(M^{\prime \prime}\right)$, which as we saw cannot occur in the same place as the corresponding transfer in $M$, cannot occur in a pair of squares disjoint from those of the perpendicular transfer preceding it either (such a transfer in disjoint squares cannot validate a relevant inequality any more than it could invalidate one in the first case considered above); a transfer towards the entry at $(i, j)$ is therefore the only possibility that remains.

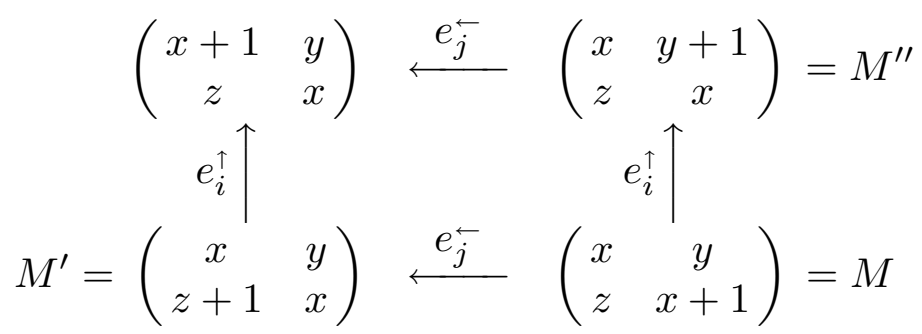

By a simple induction, the commutation lemma implies that $\left(e_{j}^{\leftarrow}\right)^{m}\left(\left(e_{i}^{\uparrow}\right)^{n}(M)\right)=$ $\left(e_{i}^{\uparrow}\right)^{n}\left(\left(e_{j}^{\leftarrow}\right)^{m}(M)\right)$ whenever $\left(e_{i}^{\uparrow}\right)^{n}(M)$ and $\left(e_{j}^{\leftarrow}\right)^{m}(M)$ are defined (this is true in the binary case as well). It is interesting to consider how this commutation is realised if one assumes that the operations applied to $M$ can each be performed by a single transfer. Much of the proof above can be applied to such transfers as well, but the final case is more complicated. One can see that transfers remain in the same position and are by the same amount, unless those in $\left(e_{i}^{\uparrow}\right)^{n}(M)$ and $\left(e_{j}^{\leftarrow}\right)^{m}(M)$ are both from the entry $M_{k+1, l+1}$ and one has $M_{k, l}<M_{k+1, l+1}<M_{k, l}+n+m$; then in fact $M_{k, l}+\max (n, m) \leq M_{k+1, l+1}$ by the assumption that $\left(e_{i}^{\uparrow}\right)^{n}(M)$ and $\left(e_{j}^{\leftarrow}\right)^{m}(M)$ are defined by single transfers. In this case one can put $d=M_{k+1, l+1}-M_{k, l}$ and decompose $\left(e_{j}^{\leftarrow}\right)^{m}=\left(e_{j}^{\leftarrow}\right)^{a} \circ\left(e_{j}^{\leftarrow}\right)^{b}$ and $\left(e_{i}^{\uparrow}\right)^{n}=\left(e_{i}^{\uparrow}\right)^{a} \circ\left(e_{i}^{\uparrow}\right)^{c}$ where $a=m+n-d$ and $b=m-a, c=n-a$; then the commutation takes place as in the diagram below, which shows the evolution of the $2 \times 2$ square at the intersection of rows $i, i+1$ and columns $j, j+1$. One sees that only in the top-left commuting square the transfers realising the same operation are between different pairs of entries. 


$$
\begin{aligned}
& \left(\begin{array}{cc}
x+a & y+c \\
z+b & x
\end{array}\right) \quad \stackrel{\left(e_{j}^{\leftarrow}\right)^{a}}{\longleftarrow}\left(\begin{array}{cc}
x & y+a+c \\
z+b & x
\end{array}\right) \stackrel{\left(e_{j}^{\leftarrow}\right)^{b}}{\longleftarrow}\left(\begin{array}{cc}
x & y+a+c \\
z & x+b
\end{array}\right)=M^{\prime \prime}
\end{aligned}
$$

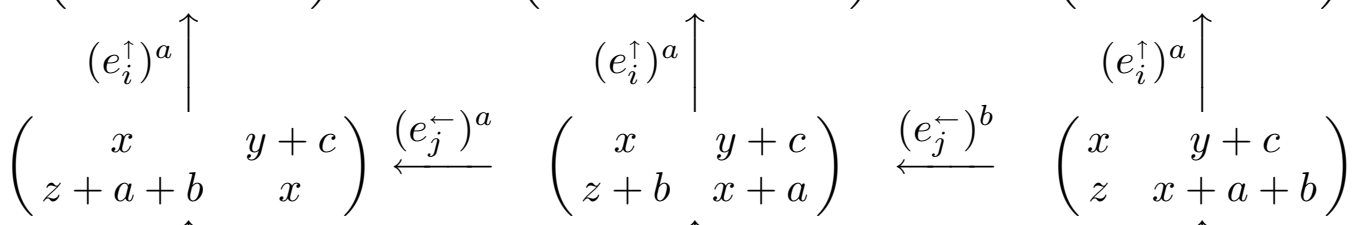

$$
\begin{aligned}
& \left(e_{i}^{\uparrow}\right)^{c \uparrow} \quad\left(e_{i}^{\uparrow}\right)^{c \uparrow} \\
& M^{\prime}=\left(\begin{array}{cc}
x & y \\
z+a+b & x+c
\end{array}\right) \stackrel{\left(e_{j}^{\leftarrow}\right)^{a}}{\longleftarrow}\left(\begin{array}{cc}
x & y \\
z+b & x+a+c
\end{array}\right) \stackrel{\left(e_{j}^{\leftarrow}\right)^{b}}{\longleftarrow}\left(\begin{array}{cc}
x & y \\
z & x+a+b+c
\end{array}\right)=M
\end{aligned}
$$

We see that when considering non-unit transfers between pairs of adjacent columns and rows, a transfer that can be realised in a single step can become a transfer broken into several steps after the commutation. The opposite can also happen, and it suffices to consider the inverse operations in the diagram to obtain an example of that situation.

\section{$\S 2$. Crystal graphs.}

We shall now discuss some basic properties of crystal operations on matrices, starting with those that involve only horizontal or only vertical operations. So for the moment we assume that one of the binary and the integral cases is chosen, as well as one of the two orientations horizontal and vertical; then raising and lowering operations can be indicated simply by $e_{i}$ and $f_{i}$, respectively. Doing so we essentially find the structure of crystal graphs of type $A_{n}$, so the results in this section are generally known in some form. Our goal here is to give an brief overview of facts, formulated for matrices, and of terminology used.

A crystal graph of matrices is a set closed under the application of operations $e_{i}$ and $f_{i}$ for $i \in \mathbf{N}$ whenever these are defined, with matrices considered as vertices, linked by directed edges defined and labelled by those operations. A connected crystal graph will be called a crystal. Since for any nonzero matrix there is always some lowering operation that can be applied, such graphs are necessarily infinite. It will in some cases be more convenient to fix some $n>0$ and to restrict our attention to matrices with at most $n$ rows (in case of vertical operations) or at most $n$ columns (in case of horizontal operations), in which case the set of crystal operations is limited to $e_{i}$ and $f_{i}$ for $i \in[n-1]$; then crystals will be finite.

Crystals have a complicated structure, but a number of properties can be established directly. Whether considering finite or infinite sets of crystal operations, it is easy to see that the crystals that occur do not depend, up to isomorphism, on whether one uses horizontal or vertical operations. In the case of integral matrices, transposition defines an isomorphism between the two structures on the set of all such matrices. In the binary case, rotation of matrices by a quarter turn counterclockwise defines an isomorphism from crystal graphs defined by vertical moves to crystal graphs defined by horizontal moves, but such a rotation can only be defined for a subset of binary matrices at a time. For any crystal for vertical moves, there is some $m \in \mathbf{N}$ so that all its nonzero matrix entries are contained in the leftmost $m$ columns, and an appropriate 


\section{Crystal graphs}

rotation maps these columns to the topmost $m$ rows in reverse order.

Using suitable rotations by a half turn, one can similarly show that when crystal operations $e_{i}$ and $f_{i}$ are limited to $i \in[n-1]$, the structure obtained from a crystal graph by interchanging the labels $e_{i}$ and $f_{n-2-i}$ is again a crystal graph of the same type. (It can be shown using other known properties that each crystal thus gives rise to a crystal isomorphic to itself, but that is not obvious at this point.)

It is also true that crystals defined using binary matrices are isomorphic to those defined using integral matrices. Here the correspondence is rather less obvious than in the above cases, so we shall state it more formally. The statement basically follows from the fact that coplactic operations on semistandard tableaux can be defined either using the Kanji reading order (down columns, from right to left), or using the Semitic reading order (backwards by rows, from top to bottom); see [vLee3, proposition 3.2.1]. Translating the operations on tableaux in terms of matrices, using binary respectively integral encodings, gives crystal operations on matrices, and leads to the following formulation.

2.1. Proposition. Let $T \in \mathrm{SST}(\lambda / \kappa)$ be a semistandard tableau, and let its binary and integral encodings be $M \in \mathcal{M}^{[2]}$ and $N \in \mathcal{M}$, respectively. Then for $m \in \mathbf{N}$, $e_{m}^{\uparrow}(M)$ is defined if and only if $e_{m}^{\leftarrow}(N)$ is defined, and if so, $e_{m}^{\uparrow}(M)$ and $e_{m}^{\leftarrow}(N)$ are respectively the binary and integral encodings of one same tableau $T^{\prime} \in \operatorname{SST}(\lambda / \kappa)$. The same is true when $e_{m}^{\uparrow}$ and $e_{m}^{\leftarrow}$ are replaced by $f_{m}^{\downarrow}$ and $f_{m}^{\vec{m}}$, respectively.

This proposition immediately implies that the correspondence of $M$ and $N$ as encodings of the same semistandard tableau of shape $\lambda / \kappa$ can be extended to a correspondence between the crystal for vertical moves that contains $M$ and the crystal for horizontal transfers that contains $N$, so that these two structures are isomorphic. Note that a matrix may satisfy the tableau condition for many different shapes (indeed any matrix satisfies infinitely many tableau conditions, though not all of them are equally interesting), so for a crystal of one type, one obtains isomorphisms with many different crystals of the other type. This indicates that it is quite common to have distinct but isomorphic crystals of matrices; that observation could also have been made after the commutation lemmas of the previous section.

When we consider in a crystal graph only the edges labelled $e_{i}$ and $f_{i}$ for a single index $i$, then as we have seen, each vertex $M$ is part of a finite ladder. The cancellations we considered in the previous section were defined, after selecting an appropriate ladder, by reversal of a lower portion of a ladder. It is natural to consider also the simpler operation of reversing each complete ladder for a fixed index $i$. This defines an involution on the set of all matrices, which in the case of vertical operations preserves all column sums while interchanging the row sums $\operatorname{row}(M)_{i}$ and $\operatorname{row}(M)_{i+1}$, and in case of horizontal operations preserves all row sums while interchanging $\operatorname{col}(M)_{i}$ and $\operatorname{col}(M)_{i+1}$. Now if one takes any crystal graph for which the multivariate generating series of its matrices by row sums (for vertical operations) or by column sums (for horizontal operations) exists (i.e., its coefficients remain finite), then that series will be invariant under all permutations of the variables; if the monomials of the series are of bounded degree, this means that the series is a symmetric function. (While the crystal graph of all binary or 
integral matrices is too large to have such a series, each individual crystal does indeed define a symmetric function.)

This is similar to the situation for Bender-Knuth involutions, which can be used not only to define cancellations, but also to show the symmetry of the weight generating series of skew semistandard tableaux, in other words of skew Schur functions. Indeed, the crystal graphs on the sets $\operatorname{Tabl}^{[2]}(\lambda / \kappa)$ or $\operatorname{Tabl}(\lambda / \kappa)$ for some skew shape $\lambda / \kappa$ are simple examples of graphs to which the symmetry result obtained by ladder reversal can be applied, and here the generating series is the skew Schur function $s_{\lambda / \kappa}\left[X_{\mathbf{N}}\right]$ in both cases. Although this result can also be obtained by using the simpler BenderKnuth involutions, there is at least one sense in which it is "better" to use the ladder reversing involutions, namely that they actually define a symmetric group action of the underlying set of any crystal graph: the ladder reversing involutions satisfy the Coxeter relations of type $A$, whereas the Bender-Knuth involutions do not. (On the other hand ladder reversal does not respect the potentials for crystal operations at other indices, so the action is not by graph isomorphisms of any kind.) This symmetric group action means that one not only knows, for any crystal graph for vertical operations and any pair of compositions $\alpha, \alpha^{\prime}$ whose parts are related by some permutation, that there are as many matrices $M$ in the crystal graph with $\operatorname{row}(M)=\alpha$ as with $\operatorname{row}(M)=\alpha^{\prime}$, but that one also has a natural bijection between those sets of matrices.

We shall now state the above matters more formally. We first define the involutions generating the action, which come in four flavours: binary or integral, and horizontal or vertical. However, like for the crystal operations, there is no explicit distinction in the notation between the binary and the integral case, so only two symbols are used, $\sigma_{i}^{\uparrow}$ for the "vertical" involutions and $\sigma_{j}^{\leftrightarrow}$ for the "horizontal" ones.

2.2. Definition. The symbols $\sigma_{i}^{\uparrow}$ and $\sigma_{j}^{\leftrightarrow}$ for $i, j \in \mathbf{N}$ denote involutions both on $\mathcal{M}^{[2]}$ and on $\mathcal{M}$, given in either case by the following expressions:

$$
\begin{gathered}
\sigma_{i}^{\uparrow}(M)=\left(e_{i}^{\uparrow}\right)^{\delta}(M) \quad \text { where } \delta=n_{i}^{\uparrow}(M)-n_{i}^{\downarrow}(M)=\operatorname{row}(M)_{i+1}-\operatorname{row}(M)_{i}, \\
\sigma_{j}^{\leftrightarrow}(M)=\left(e_{j}^{\leftarrow}\right)^{\delta}(M) \quad \text { where } \delta=n_{j}^{\leftarrow}(M)-n_{j}(M)=\operatorname{col}(M)_{j+1}-\operatorname{col}(M)_{j} .
\end{gathered}
$$

and where $\left(e_{i}^{\uparrow}\right)^{\delta}$ and $\left(e_{j}^{\leftarrow}\right)^{\delta}$ are to be interpreted as $\left(f_{i}^{\downarrow}\right)^{-\delta}$ and $\left(f_{j}\right)^{-\delta}$, respectively, when $\delta<0$, and as the identity when $\delta=0$.

It is immediately obvious that one has the following identities, where $s_{i}$ denotes the transposition of $i$ and $i+1$, acting as usual on compositions by permuting their parts:

$$
\begin{aligned}
\operatorname{row}\left(\sigma_{i}^{\ddagger}(M)\right) & =s_{i} \cdot \operatorname{row}(M) \\
\operatorname{col}\left(\sigma_{i}^{\ddagger}(M)\right) & =\operatorname{col}(M)
\end{aligned} \quad \text { and } \quad \begin{aligned}
\operatorname{row}\left(\sigma_{j}^{\leftrightarrow}(M)\right) & =\operatorname{row}(M) \\
\operatorname{col}\left(\sigma_{j}^{\leftrightarrow}(M)\right) & =s_{j} \cdot \operatorname{col}(M)
\end{aligned}
$$

Moreover, when $\operatorname{row}(M)_{i}=\operatorname{row}(M)_{i+1}$ one has $\sigma_{i}^{\uparrow}(M)=M$, and similarly one has $\sigma_{j}^{\leftrightarrow}(M)=M$ when $\operatorname{col}(M)_{j}=\operatorname{col}(M)_{j+1}$; this contrasts with the Bender-Knuth involutions, which may send one tableau whose weight already has equal values at the positions being interchanged to another such tableau. It follows from the commutation lemmas that $\sigma_{i}^{\uparrow}$ and $\sigma_{j}^{\leftrightarrow}$ commute for all $i, j \in \mathbf{N}$. 


\section{Crystal graphs}

2.3. Lemma. The operations $\sigma_{i}^{\uparrow}$ satisfy the Coxeter relations $\left(\sigma_{i}^{\uparrow}\right)^{2}=1, \quad \sigma_{i}^{\uparrow} \sigma_{k}^{\downarrow}=$ $\sigma_{k}^{\uparrow} \sigma_{i}^{\uparrow}$ when $|k-i| \geq 2$, and $\sigma_{i}^{\uparrow} \sigma_{i+1}^{\uparrow} \sigma_{i}^{\uparrow}=\sigma_{i+1}^{\uparrow} \sigma_{i}^{\uparrow} \sigma_{i+1}^{\uparrow}$. Similar relations hold for the operations $\sigma_{j}^{\leftrightarrow}$.

Via the correspondence of crystal operations to coplactic operations on semistandard tableaux, this lemma is essentially equivalent to [Loth, theorem 5.6.3], but we shall give a proof at the end of our paper. The following theorem summarises the main conclusions that follow from the relations given.

2.4. Theorem. There are two commuting actions of the group $\mathbf{S}_{\infty}$, denoted by $\pi^{\ddagger}(M)$ and $\pi^{\leftrightarrow}(M)$, on each of $\mathcal{M}^{[2]}$ and $\mathcal{M}$; they are defined in both cases by $s_{i}^{\ddagger}(M)=\sigma_{i}^{\downarrow}(M)$ and $s_{i}^{\leftrightarrow}(M)=\sigma_{i}^{\leftrightarrow}(M)$ for a generator $s_{i}$ of $\mathbf{S}_{\infty}$ (the transposition $(i, i+1)$ ). Any operation $\pi^{\downarrow}$ permutes the parts of $\operatorname{row}(M)$ by $\pi$ while leaving $\operatorname{col}(M)$ invariant, and if $\pi$ fixes $\operatorname{row}(M)$ then $\pi^{\downarrow}$ fixes $M$; similar statements hold for $\pi^{\leftrightarrow}$.

Proof. The only point that is not immediate is the final claim that $\pi^{\downarrow}$ fixes $M$, which only follows directly from the observed properties of $\sigma_{i}^{\uparrow}$ and $\sigma_{j}^{\leftrightarrow}$ in case the stabiliser of $\operatorname{row}(M)$ is generated by a subset of the generators $s_{i}$ (i.e., any equalities among the parts of $\operatorname{row}(M)$ occur in consecutive ranges). But that stabiliser is always conjugate to such a (parabolic) subgroup, which allows reduction to the given case.

We continue with some simpler but important observations. While every matrix admits some lowering operation, both vertically and horizontally, there are matrices that permit no raising operations in a given direction at all. Indeed it follows from propositions 1.3 .5 and 1.4 .5 that a $M \in \mathcal{M}^{[2]}$ or $N \in \mathcal{M}$ admits no upwards operations if and only if $M \in \operatorname{LR}^{[2]}(\nu /(0))$ where $\nu=\operatorname{col}(M) \in \mathcal{P}$ respectively $N \in \operatorname{Tabl}(\lambda /(0))$ where $\lambda=\operatorname{col}(M) \in \mathcal{P}$, while the matrix admits no leftwards operations if and only if $M \in \operatorname{Tabl}^{[2]}(\lambda /(0))$ respectively $N \in \operatorname{LR}(\nu /(0))$, where $\lambda=\operatorname{row}(M)$ and $\nu=\operatorname{row}(N)$. The given values of $\lambda$ and $\nu$ are the only ones for which the respective conditions could hold, so these properties state that the fact that no raising operations are possible means that the matrix encodes, according to the case considered, a semistandard tableau of straight shape, or some (skew) Littlewood-Richardson tableau.

Fixing one direction as before, if we start with a given matrix, and repeat the step of applying some raising operation that can be applied, until no such operation exists, then we end up with a matrix of the type just described. The process cannot go on indefinitely since at every step we move a unit to a row or column with a lower number, which decreases an obvious statistic (the sum over all units of their row respectively column number). The matrix found at the end of the process will be said to be obtained from the original matrix by exhausting raising operations (of the type considered). We shall show later that the final matrix found does not depend on the choices made during the exhaustion process (in other words, the process is confluent). This is an important and quite nontrivial statement; it implies that every crystal contains a unique vertex in which no raising operations are possible. For the moment however it suffices to know that some final matrix can always be reached in this way.

Similar properties are known to hold for jeu de taquin on skew semistandard tableaux, which can be used to "rectify" a skew tableau to one of straight shape by a 
nondeterministic procedure, but whose final result is independent of the choices made. In fact there is a close relation between that game and crystal operations on matrices encoding the tableaux. It is known, see [vLee3, theorem 3.4.2], that jeu de taquin slides applied to a tableau $T$ correspond to sequences of coplactic operations applied to a its "companion tableau", which is a tableau whose integral encoding is the transpose of the integral encoding of $T$. Since coplactic operations on tableaux correspond to horizontal crystal operations on their integral encodings, this means that jeu de taquin slides correspond to sequences of vertical crystal operations. A correspondence exists also for binary encodings. More precisely, one has the following.

2.5. Proposition. Let $T \in \operatorname{SST}(\lambda / \kappa)$ and let $T^{\prime} \in \operatorname{SST}\left(\lambda^{\prime} / \kappa^{\prime}\right)$ be obtained from $T$ by an inward jeu de taquin slide starting at the corner $\left(i_{0}, j_{0}\right)$ of $\kappa$ and ending in the corner $\left(i_{1}, j_{1}\right)$ of $\lambda$. Then their respective binary encodings $M, M^{\prime}$ are related by $M^{\prime}=e_{j_{1}-1}^{\leftarrow}\left(\cdots\left(e_{j_{0}+1}^{\leftarrow}\left(e_{j_{0}}^{\leftarrow}(M)\right) \cdots\right)\right)$, and their respective integral encodings $N, N^{\prime}$ are related by $N^{\prime}=e_{i_{1}-1}^{\uparrow}\left(\cdots\left(e_{i_{0}+1}^{\uparrow}\left(e_{i_{0}}^{\uparrow}(N)\right) \cdots\right)\right)$.

Proof. In the description of a jeu de taquin slide in terms of a skew tableau with an empty square into which entries as successively slid, one can at each point associate both a binary and an integral encoding matrix, which count the entries in columns respectively rows in the usual fashion (ignoring the empty square). It is a straightforward verification that under this correspondence each leftward slide of a tableau entry results in applying a leftward move in corresponding pair of columns of the binary matrix while leaving the integral matrix unchanged, and that each upward slide of a tableau entry results in applying an upward unit transfer in corresponding pair of rows of the integral matrix while leaving the binary matrix unchanged. Indeed, the inequalities in definition 1.3.1b reflect the fact that the entries above and below a horizontally sliding entry, in the same pair of columns, satisfy weak increase along rows, while the inequalities in definition 1.4.1a reflect the fact that the entries to the left and right of a vertically sliding entry, and in the same rows, satisfy strict increase down columns. The proposition immediately follows.

Thus one can see that if a skew tableau $T$ has rectification $P$, then the binary encoding of $P$ can be obtained from that of $T$ by exhausting leftward moves, while the integral encoding of $P$ can be obtained from that of $T$ by exhausting upward transfers. However this reasoning does not yet imply that these are the only possible results of such exhaustion of raising operations, even if one admits the uniqueness of the rectification of $T$, since jeu de taquin slides correspond only to specific composition of raising operations, and there might exist transitions that can be realised by raising operations but not by jeu de taquin.

Since the raising operations on matrices in the direction perpendicular to the one just considered correspond to coplactic operations on tableaux, one easily recognises a relation between the commutation lemmas of the previous section and the commutation of coplactic operations with jeu de taquin, as stated for instance in [vLee3, theorem 3.3.1]. In the next section we shall elaborate on this relation, developing a theory for matrices that is similar (but more symmetric) than the one for semistandard tableaux. 


\section{Double crystals}

\section{$\S 3$. Double crystals.}

We shall now return to considering at the same time vertical and horizontal operations. A set of all matrices that can be obtained from a given one by using both vertical and horizontal crystal operations, provided with the structure of labelled directed graph defined by those operations, will be called a double crystal. Due to the commutation lemmas, double crystals will be much easier to study than ordinary crystals. We shall see that they can be used to define in a very natural way correspondences quite similar to the RSK correspondence and to its dual correspondence that was (also) defined in [Knu], respectively. In fact, the correspondence that is most naturally obtained for integral matrices is the Burge variant of the RSK correspondence, described in [Fult, A.4.1].

\subsection{Knuth correspondences for binary and integral matrices.}

We have just seen that by exhausting either vertical or horizontal raising operations, one can transform any integral or binary matrix into one that encodes, according to the case considered, a straight semistandard tableau or a Littlewood-Richardson tableau. Now by exhausting both vertical and horizontal raising operations, one can obviously transform any integral or binary matrix into one that encodes a Littlewood-Richardson tableau of straight shape. It is well known that the possibilities for such tableaux are extremely limited: in their display, any row $i$ can contain only entries $i$, whence such tableaux are completely determined by either their shape or their weight, which moreover are the same partition $\lambda$. One easily sees that the binary and integral encodings of such tableaux are the matrices described by the following definition.

3.1.1. Definition. Let $\lambda \in \mathcal{P}$. We denote by $\operatorname{Diag}^{[2]}(\lambda) \in \mathcal{M}^{[2]}$ the binary matrix $([(i, j) \in[\lambda]])_{i, j \in \mathbf{N}}$ whose bits ' 1 ' occur precisely in the shape of the Young diagram $[\lambda]$. We denote by $\operatorname{Diag}(\lambda) \in \mathcal{M}$ the integral matrix $=\left([i=j] \lambda_{i}\right)_{i, j \in \mathbf{N}}$, which is diagonal with the parts of $\lambda$ as diagonal entries.

One immediately sees that this binary matrix satisfies $\operatorname{row}\left(\operatorname{Diag}^{[2]}(\lambda)\right)=\lambda$ and $\operatorname{col}\left(\operatorname{Diag}^{[2]}(\lambda)\right)=\lambda^{t}$, while this integral matrix satisfies $\operatorname{row}(\operatorname{Diag}(\lambda))=\operatorname{col}(\operatorname{Diag}(\lambda))=$ $\lambda$. From the consideration of tableaux one may deduce that these matrices are precisely the ones for which all vertical and horizontal raising operations are exhausted, but it is easy and worth while to prove this directly.

3.1.2. Lemma. A binary matrix $M \in \mathcal{M}^{[2]}$ satisfies $\forall i: n_{i}^{\uparrow}(M)=0$ and $\forall j: n_{j}^{\leftarrow}(M)=0$ if and only if $M=\operatorname{Diag}^{[2]}(\lambda)$ for some $\lambda \in \mathcal{P}$. Similarly an integral matrix $N \in \mathcal{M}$

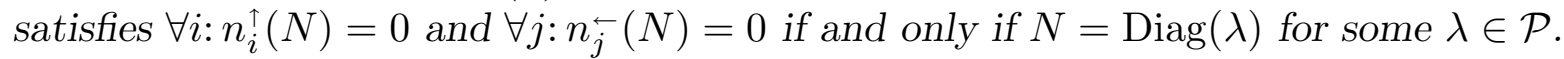

Proof. One easily sees that no raising operations are possible for $\operatorname{Diag}^{[2]}(\lambda)$ or for $\operatorname{Diag}(\lambda)$, since for $\operatorname{Diag}^{[2]}(\lambda)$ there are not even candidates for upward of leftward moves, while for $N=\operatorname{Diag}(\lambda)$ and all $i$ the units of $N_{i+1, i+1}$ are blocked by those of $N_{i, i}$ for upward or leftward transfers. This takes care of the "if" parts. For the "only if" parts we shall apply induction on the sum of all matrix entries, the case of null matrices being obvious (with $\lambda=(0)$ ).

Suppose that $\forall i: n_{i}^{\uparrow}(M)=0$ and $\forall j: n_{j}^{\leftarrow}(M)=0$, and let $(k, l)$ be the indices of the bit $M_{k, l}=1$ for which $l$ is maximal, and $k$ is minimal for that $l$. If $k>0$ one would have 
$n_{k-1}^{\uparrow}(M)>0$, so $k=0$. But then also $M_{0, j}=1$ for all $j<l$, since otherwise the largest $j$ violating this would give $n_{j}^{\leftarrow}(M)>0$. Let $M^{\prime}=\left(M_{i+1, j}\right)_{i, j \in \mathbf{N}}$ be the matrix obtained by removing the topmost row $M_{0}$. The hypothesis $n_{j}^{\leftarrow}(M)=0$ implies $n_{j}^{\leftarrow}\left(M^{\prime}\right)=0$ for $j<l$ since $\left(M_{0, j}, M_{0, j+1}\right)=(1,1)$; one also has $n_{j}^{\leftarrow}\left(M^{\prime}\right)=0$ for $j \geq l$, for lack of any bits $M_{i, j+1}^{\prime}=1$. Therefore $M^{\prime}$ satisfies the hypotheses of the lemma, and by induction one has $M^{\prime}=\operatorname{Diag}^{[2]}\left(\lambda^{\prime}\right)$ for some $\lambda^{\prime} \in \mathcal{P}$, with obviously $\lambda_{0}^{\prime} \leq l$. Then $M=\operatorname{Diag}^{[2]}(\lambda)$, where $\lambda$ is the partition obtained by prefixing $l$ to the parts of $\lambda^{\prime}$ (formally: $\lambda_{0}=l$ and $\lambda_{i+1}=\lambda_{i}^{\prime}$ for $i \in \mathbf{N}$ ).

Now suppose that $\forall i: n_{i}^{\uparrow}(N)=0$ and $\forall j: n_{j}^{\leftarrow}(N)=0$ (the integral case). The conditions $n_{i}^{\uparrow}(N)=0$ and $n_{j}^{\leftarrow}(N)=0$ respectively imply that $N_{i+1,0}=0$ and $N_{0, j+1}=0$. But one cannot have $\operatorname{row}(N)_{0}=0$, since then for the smallest index $i$ such that $\operatorname{row}(N)_{i+1}>0$ one would have $n_{i}^{\uparrow}(N)=\operatorname{row}(N)_{i+1}>0$. Therefore $N_{0,0}>0$ and we can apply induction to the matrix $N^{\prime}=\left(N_{i+1, j+1}\right)_{i, j \in \mathbf{N}}$ obtained by removing the initial row and column from $N$, since clearly $n_{i}^{\uparrow}\left(N^{\prime}\right)=n_{i+1}^{\uparrow}(N)=0$ and $n_{j}^{\leftarrow}\left(N^{\prime}\right)=n_{j+1}^{\leftarrow}(N)=0$. Then $N^{\prime}=\operatorname{Diag}\left(\lambda^{\prime}\right)$ for some $\lambda^{\prime} \in \mathcal{P}$, and $N_{0,0} \geq \lambda_{0}^{\prime}$ follows either from $n_{0}^{\uparrow}(N)=0$ or from $n_{0}^{\leftarrow}(N)=0$. So one has $N=\operatorname{Diag}(\lambda)$, where $\lambda \in \mathcal{P}$ is obtained by prefixing $N_{0,0}$ to the parts of $\lambda^{\prime}$.

Now that we have characterised the matrices at which the process of applying raising operations can stop, and with the commutation lemmas at our disposal, the general structure of double crystals can be easily analysed, as follows.

Fix a matrix $M \in \mathcal{M}^{[2]}$ or $M \in \mathcal{M}$. Suppose that $R^{\uparrow}$ is some sequence of raising operations $e_{i}^{\uparrow}$ for varying $i \in \mathbf{N}$ that exhausts such operations when applied to $M$, in other words such that the resulting matrix $R^{\uparrow}(M)$ satisfies $\forall i: n_{i}^{\uparrow}\left(R^{\uparrow}(M)\right)=0$. Similarly let $R^{\leftarrow}$ be some sequence of raising operations $e_{j}^{\leftarrow}$ for varying $j \in \mathbf{N}$ that exhausts such operations when applied to $M$; then the resulting matrix $R^{\leftarrow}(M)$ satisfies $\forall j: n_{j}^{\leftarrow}\left(R^{\leftarrow}(M)\right)=0$. By lemma 1.3.7 or lemma 1.4.7, the expressions $R^{\leftarrow}\left(R^{\uparrow}(M)\right)$ and $R^{\uparrow}\left(R^{\leftarrow}(M)\right)$ are both defined and they designate the same matrix, which we shall call $N$. By proposition 1.3.6 or 1.4.6, one has $\forall i: n_{i}^{\uparrow}(N)=0$ and $\forall j: n_{j}^{\leftarrow}(N)=0$, and so lemma 3.1.2 states that $N=\operatorname{Diag}^{[2]}(\lambda)$ or $N=\operatorname{Diag}(\lambda)$ (according to the case considered) for some $\lambda \in \mathcal{P}$. Since $R^{\leftarrow}$ preserves row sums one has $\operatorname{row}\left(R^{\uparrow}(M)\right)=\operatorname{row}(N)$, which composition equals $\lambda$ both in the binary and in the integral case; similarly $R^{\uparrow}$ preserves column sums, so one has $\operatorname{col}\left(R^{\leftarrow}(M)\right)=\operatorname{col}(N)$, which equals $\lambda$ in the integral case and $\lambda^{t}$ in the binary case. Thus either of the values $\operatorname{row}\left(R^{\uparrow}(M)\right)$ or $\operatorname{col}\left(R^{\leftarrow}(M)\right)$ completely determines $\lambda$.

Now let $S^{\uparrow}$ and $S^{\leftarrow}$ be other sequences of upward respectively leftward crystal operations that can be applied to $M$ and that exhaust such operations. Then the matrices $S^{\leftarrow}\left(R^{\uparrow}(M)\right)$ and $S^{\uparrow}\left(R^{\leftarrow}(M)\right)$ satisfy the conditions of lemma 3.1.2, and since their row sums respectively their column sums are the same as those of $N$, they must both be equal to $N$. From $R^{\leftarrow}$ we can form the "inverse" operation $R^{\rightarrow}$ by composing the lowering operations $f_{j}$ corresponding to the operations $e_{j}^{\leftarrow}$ used, in the opposite order. Applying $R^{\rightarrow}$ to the equation $R^{\leftarrow}\left(R^{\uparrow}(M)\right)=N=S^{\uparrow}\left(R^{\leftarrow}(M)\right)=R^{\leftarrow}\left(S^{\uparrow}(M)\right)$, one finds $R^{\uparrow}(M)=S^{\uparrow}(M)$, and one similarly finds $R^{\leftarrow}(M)=S \leftarrow(M)$. So the matrices obtained from $M$ by exhausting vertical or horizontal crystal operations are uniquely 


\subsection{Knuth correspondences for binary and integral matrices}

defined. The matrix $N$ obtained by exhausting both types of operations is also uniquely determined by $M$, and we shall call $N$ the normal form of $M$.

Moreover from the knowledge of $R^{\uparrow}(M)$ and $R^{\leftarrow}(M)$ one can uniquely reconstruct $M$. To state this more precisely, let $P$ and $Q$ be matrices of the same type (binary or integral) that satisfy $\forall i: n_{i}^{\uparrow}(P)=0$ and $\forall j: n_{j}^{\leftarrow}(Q)=0$ (which implies that $\operatorname{row}(P)$ and $\operatorname{col}(Q)$ are partitions), and that satisfy moreover $\operatorname{row}(P)=\operatorname{col}(Q)^{t}$ in the binary case, or $\operatorname{row}(P)=\operatorname{col}(Q)$ in the integral case. Let $R^{\leftarrow}(P)$ and $R^{\uparrow}(Q)$ be obtained by from $P$ by exhausting leftward crystal operations, respectively from $Q$ by exhausting upwards crystal operations (as before $R^{\leftarrow}$ and $R^{\uparrow}$ denote particular sequences of operations that realise these transformations). Both matrices satisfy the conditions of lemma 3.1.2, and by the hypothesis on column and row sums, the partition $\lambda$ in the conclusion of that lemma is the same in both cases; therefore $R^{\leftarrow}(P)=R^{\uparrow}(Q)$. As before we can form inverse operations $R^{\rightarrow}$ and $R^{\downarrow}$ of $R^{\leftarrow}$ and of $R^{\uparrow}$, respectively. We successively apply these inverse operations to the equation $R^{\leftarrow}(P)=R^{\uparrow}(Q)$, and by commutation of $R^{\rightarrow}$ and $R^{\downarrow}$ (which follows that of $R^{\leftarrow}$ and $R^{\uparrow}$ ) one finds $R^{\downarrow}(P)=R^{\rightarrow}(Q)$ ). In the members of this final equation we have found a matrix $M$ for which $R^{\uparrow}(M)=P$ and $R^{\leftarrow}(M)=Q$.

To show uniqueness of $M$, suppose that $M^{\prime}$ is another matrix, and that $S^{\uparrow}$ and $S^{\leftarrow}$ are other sequences of upwards respectively leftwards crystal operations for which $P=S^{\uparrow}\left(M^{\prime}\right)$ and $Q=S^{\leftarrow}\left(M^{\prime}\right)$. Then $S^{\leftarrow}(P)$ is the normal form of $P$, and therefore equal to $R^{\leftarrow}(P)$. From this equality and $P=S^{\uparrow}\left(M^{\prime}\right)$ one deduces by commutation that $S^{\uparrow}\left(S^{\leftarrow}\left(M^{\prime}\right)\right)=S^{\uparrow}\left(R^{\leftarrow}\left(M^{\prime}\right)\right)$, and since $S^{\leftarrow}\left(M^{\prime}\right)=Q=R^{\leftarrow}(M)$ this gives $S^{\uparrow}\left(R^{\leftarrow}(M)\right)=S^{\uparrow}\left(R^{\leftarrow}\left(M^{\prime}\right)\right)$. But $S^{\uparrow}$ and $R^{\leftarrow}$ are invertible operations, so it follows that $M=M^{\prime}$.

The above reasoning shows the extraordinary usefulness of the commutation lemmas. But despite the relative ease with which the conclusions are reached, one should not forget that each application of a crystal operation has to be justified; for instance in the final part we could talk about $S^{\leftarrow}(P)$ only because $P=S^{\uparrow}\left(M^{\prime}\right)$ and by assumption $S \leftarrow\left(M^{\prime}\right)$ is defined. Also it should not be inferred from $S^{\leftarrow}(P)=R \leftarrow(P)$ that $S \leftarrow$ and $R^{\leftarrow}$ are equivalent sequences of crystal operations, or even that they can be applied to the same set of matrices. We now state more formally the statement that follows from the argument given.

3.1.3. Theorem. (binary and integral decomposition theorem)

(1) There is a bijective correspondence between binary matrices $M \in \mathcal{M}^{[2]}$ on one hand, and pairs $P, Q \in \mathcal{M}^{[2]}$ of such matrices satisfying $\forall i: n_{i}^{\uparrow}(P)=0$ and $\forall j: n_{j}^{\leftarrow}(Q)=0$ and $\operatorname{row}(P)=\operatorname{col}(Q)^{t}$ on the other hand, which is determined by the condition that $P$ can be obtained from $M$ by a finite sequence of operations chosen from $\left\{e_{i}^{\uparrow} \mid i \in \mathbf{N}\right\}$ and $Q$ can be obtained from $M$ by applying a finite sequence of operations chosen from $\left\{e_{j}^{\leftarrow} \mid j \in \mathbf{N}\right\}$. In particular $\operatorname{col}(P)=\operatorname{col}(M)$ and $\operatorname{row}(Q)=$ $\operatorname{row}(M)$.

(2) There is a bijective correspondence between integral matrices $M \in \mathcal{M}$ on one hand, and pairs $P, Q \in \mathcal{M}$ of such matrices satisfying $\forall i: n_{i}^{\uparrow}(P)=0$ and $\forall j: n_{j}^{\leftarrow}(Q)=0$ and $\operatorname{row}(P)=\operatorname{col}(Q)$ on the other hand, which is determined by the condition 


\subsection{Knuth correspondences for binary and integral matrices}

that $P$ can be obtained from $M$ by a finite sequence of operations chosen from $\left\{e_{i}^{\uparrow} \mid i \in \mathbf{N}\right\}$ and $Q$ can be obtained from $M$ by applying a finite sequence of operations chosen from $\left\{e_{j}^{\leftarrow} \mid j \in \mathbf{N}\right\}$. In particular $\operatorname{col}(P)=\operatorname{col}(M)$ and $\operatorname{row}(Q)=$ $\operatorname{row}(M)$.

These correspondences can be explicitly computed by the constructions given above.

3.1.4. Corollary. The crystal defined by any binary or integral matrix, and by one type (vertical or horizontal) of crystal operations, contains a unique vertex at which the potentials for all raising operations are zero. Moreover, the crystal is determined up to isomorphism by the potentials for the lowering operations at that vertex.

Proof. We have seen that exhausting upward or leftward crystal operations in all possible ways from a given initial matrix always leads to the same final matrix (the matrix $P$ respectively $Q$ of theorem 3.1.3), where the potentials for all raising operations are zero. Then whenever two matrices $M, M^{\prime}$ are linked by a crystal operation in the chosen direction, this final matrix will be the same whether $M$ or $M^{\prime}$ is taken as point of departure. Consequently, every matrix in the crystal considered leads to the same final matrix, which is the unique matrix in the crystal where the potentials for all raising operations are zero.

For the final part of the claim, consider the common normal form $N=\operatorname{Diag}^{[2]}(\lambda)$ or $N=\operatorname{Diag}(\lambda)$ of each of $M, P$, and $Q$ in theorem 3.1.3. Since horizontal crystal operations induce isomorphisms of crystals defined by vertical crystal operations (due to their commutation) and vice versa, the crystal for vertical operations containing $P$ is isomorphic to the one for the same operations containing $N$, and similarly for the crystal for horizontal operations containing $Q$; it will suffice to show that $\lambda$ can be deduced from the sequences of potentials for the appropriate lowering operations at $P$ respectively from those at $Q$. Now $n_{i}^{\downarrow}(P)=n_{i}^{\downarrow}(N)=\lambda_{i}-\lambda_{i+1}$ for all $i$ in both the binary and the integral case, while $n_{j}(Q)=n_{j}(N)$ for all $j$, which equals $\lambda_{j}^{t}-\lambda_{j+1}^{t}$ in the binary case, or $\lambda_{j}-\lambda_{j+1}$ in the integral case. Since $\lambda$ can be reconstructed from the sequence of differences its consecutive parts or of those of $\lambda^{t}$ (for instance $\lambda_{k}=\sum_{i \geq k} d_{i}$ for all $k \in \mathbf{N}$, where $\left.d_{i}=\lambda_{i}-\lambda_{i+1}\right)$, this completes the proof.

Part (2) of the theorem is very similar to the statement of bijectivity of the RSK correspondence (see for instance [Stan, theorem 7.11.5]), since the matrices $P$ and $Q^{t}$ both lie in $\operatorname{Tabl}(\lambda /(0))$ where $\lambda$ is the partition $\operatorname{row}(P)=\operatorname{col}(Q)$, whence they encode straight semistandard tableaux of equal shape $\lambda$; the statements $\operatorname{col}(P)=\operatorname{col}(M)$ and $\operatorname{row}(Q)=\operatorname{row}(M)$ give the usual relation between the weights of these tableaux and the column and row sums of $M$. However, the construction of the bijection is quite different, and as we shall see, the bijection itself does not correspond directly to the RSK correspondence either. Yet it is appropriate to call the bijection of part (2) a "Knuth correspondence". This is a generic term for a large class of generalisations of the RSK correspondence that was used in [Fom] and in [vLee4], without being formally defined. It actually refers to the result of the type of construction defined in [Fom] using growth diagrams; that construction is rather different from the one above, but as we shall see below, the bijection considered does allow an alternative construction using growth diagrams. 


\subsection{Relation with jeu de taquin and with Robinson's correspondence}

As for the bijection of Part 1, it has some resemblance to the dual RSK correspondence, the second bijection that was defined together with the usual RSK correspondence in $[\mathrm{Knu}]$. That correspondence is a bijection between binary matrices and pairs of straight tableaux that are less symmetric than those associated to integral matrices: one can define them to be both semistandard but of transpose shapes (as in the original paper), or one can define one of them to be semistandard and the other transpose semistandard, in which case their shapes will be equal (as in [Stan, §7.14]); in fact at the end of the original construction one of the tableaux is transposed to make both semistandard. In our bijection however, while $Q$ is the binary encoding of a straight semistandard tableau of shape $\lambda=\operatorname{col}(Q)^{t}$, it is not entirely natural to associate a straight semistandard or transpose semistandard tableau to $P$. One could rotate $P$ a quarter turn counterclockwise to get the binary encoding of a semistandard tableau of shape row $(P)^{t}=\lambda^{t}$, but that matrix, and the weight of the tableau it encodes, depend on the number of columns of the matrix that is rotated, which should be large enough to contain all bits ' 1 ' of $P$. The type of straight tableau most naturally associated to $P$ is a reverse transpose semistandard tableau of shape $\operatorname{row}(P)=\lambda$, namely the tableau given by the sequence of partitions $\left(\lambda^{(l)}\right)_{l \in \mathbf{N}}$ where $\lambda^{(l)}=\sum_{j \geq l} P_{j}^{t}$.

\subsection{Relation with jeu de taquin and with Robinson's correspondence.}

Let us give an example of the bijections of theorem 3.1.3. We could take arbitrary matrices $M$ in both cases, but it will be instructive to use the binary and integral encodings of a same semistandard tableau $T$. We shall take

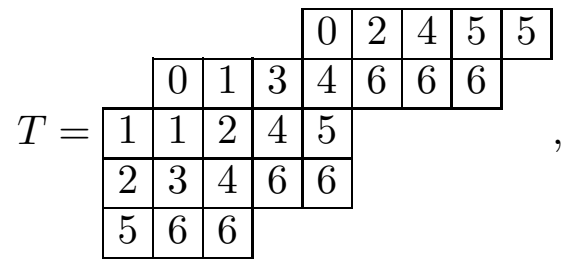

that was also used as illustration in $\S 1.1$. Taking its binary encoding $M$ in theorem 3.1.3(1), one has 


$$
\begin{aligned}
N=\left(\begin{array}{lllllllll}
1 & 1 & 1 & 1 & 1 & 1 & 1 & 1 & 0 \\
1 & 1 & 1 & 1 & 1 & 1 & 1 & 1 & 0 \\
1 & 1 & 1 & 1 & 1 & 0 & 0 & 0 & 0 \\
1 & 1 & 1 & 0 & 0 & 0 & 0 & 0 & 0 \\
1 & 0 & 0 & 0 & 0 & 0 & 0 & 0 & 0 \\
0 & 0 & 0 & 0 & 0 & 0 & 0 & 0 & 0 \\
0 & 0 & 0 & 0 & 0 & 0 & 0 & 0 & 0
\end{array}\right) \stackrel{R}{\longleftarrow}\left(\begin{array}{llllllllll}
1 & 1 & 1 & 0 & 1 & 1 & 1 & 1 & 1 \\
1 & 1 & 1 & 1 & 1 & 1 & 1 & 1 & 0 \\
1 & 1 & 1 & 1 & 1 & 0 & 0 & 0 & 0 \\
0 & 1 & 0 & 1 & 1 & 0 & 0 & 0 & 0 \\
0 & 0 & 1 & 0 & 0 & 0 & 0 & 0 & 0 \\
0 & 0 & 0 & 0 & 0 & 0 & 0 & 0 & 0 \\
0 & 0 & 0 & 0 & 0 & 0 & 0 & 0 & 0
\end{array}\right)=P \\
Q=\left(\begin{array}{llllllllll}
1 & 1 & 0 & 0 & 0 & 0 & 0 & 0 & 0 \\
1 & 1 & 1 & 0 & 0 & 0 & 0 & 0 & 0 \\
1 & 1 & 0 & 1 & 0 & 0 & 0 & 0 & 0 \\
1 & 0 & 1 & 0 & 0 & 0 & 0 & 0 & 0 \\
0 & 0 & 1 & 1 & 1 & 1 & 0 & 0 & 0 \\
1 & 0 & 0 & 0 & 1 & 0 & 1 & 1 & 0 \\
0 & 1 & 1 & 1 & 1 & 1 & 1 & 1 & 0
\end{array}\right) \stackrel{R}{\longleftarrow}\left(\begin{array}{llllllllll}
0 & 1 & 0 & 0 & 1 & 0 & 0 & 0 & 0 \\
1 & 1 & 1 & 0 & 0 & 0 & 0 & 0 & 0 \\
1 & 0 & 1 & 0 & 0 & 1 & 0 & 0 & 0 \\
0 & 1 & 0 & 1 & 0 & 0 & 0 & 0 & 0 \\
0 & 0 & 1 & 1 & 1 & 0 & 1 & 0 & 0 \\
1 & 0 & 0 & 0 & 1 & 0 & 0 & 1 & 1 \\
0 & 1 & 1 & 1 & 1 & 1 & 1 & 1 & 0
\end{array}\right)=M,
\end{aligned}
$$

where the composite raising operations are for instance $R^{\leftarrow}=e_{7}^{\leftarrow} e_{6}^{\leftarrow} e_{5}^{\leftarrow} e_{2}^{\leftarrow} e_{3}^{\leftarrow} e_{4}^{\leftarrow} e_{0}^{\leftarrow} e_{1}^{\leftarrow}$ $e_{2}^{\leftarrow} e_{3}^{\leftarrow} e_{1}^{\leftarrow} e_{0}^{\leftarrow}$ and $R^{\uparrow}=\left(e_{1}^{\uparrow}\right)^{3}\left(e_{2}^{\uparrow}\right)^{5}\left(e_{3}^{\uparrow}\right)^{7}\left(e_{4}^{\uparrow}\right)^{7}\left(e_{5}^{\uparrow}\right)^{7}\left(e_{0}^{\uparrow}\right)^{2}\left(e_{1}^{\uparrow}\right)^{3}\left(e_{2}^{\uparrow}\right)^{3}\left(e_{3}^{\uparrow}\right)^{3}\left(e_{4}^{\uparrow}\right)^{4}\left(e_{0}^{\uparrow}\right)^{2}\left(e_{1}^{\uparrow}\right)^{3}$ $\left(e_{2}^{\uparrow}\right)^{4}\left(e_{3}^{\uparrow}\right)^{4} e_{1}^{\uparrow} e_{2}^{\uparrow} e_{0}^{\uparrow} e_{1}^{\uparrow} e_{0}^{\uparrow}$; as usual operations are applied from right to left, and we have systematically chosen to climb the ladder with the lowest possible index first, and to climb it completely (so without switching ladders when a lower index ladder becomes available halfway up). As an illustration of theorem 3.1.3(2), taking for $M$ the integral encoding of $T$, one has the diagram

$$
\begin{aligned}
N=\left(\begin{array}{lllllll}
8 & 0 & 0 & 0 & 0 & 0 & 0 \\
0 & 8 & 0 & 0 & 0 & 0 & 0 \\
0 & 0 & 5 & 0 & 0 & 0 & 0 \\
0 & 0 & 0 & 3 & 0 & 0 & 0 \\
0 & 0 & 0 & 0 & 1 & 0 & 0
\end{array}\right) & R^{\leftarrow} \\
& R^{\uparrow}
\end{aligned}
$$

where the composite raising operations are for instance (using the same rule to choose ladders) given by $R^{\leftarrow}=\left(e_{1}^{\leftarrow}\right)^{3}\left(e_{2}^{\leftarrow}\right)^{5}\left(e_{3}^{\leftarrow}\right)^{7}\left(e_{4}^{\leftarrow}\right)^{7}\left(e_{5}^{\leftarrow}\right)^{7}\left(e_{0}^{\leftarrow}\right)^{2}\left(e_{1}^{\leftarrow}\right)^{3}\left(e_{2}^{\leftarrow}\right)^{3}\left(e_{3}^{\leftarrow}\right)^{3}\left(e_{4}^{\leftarrow}\right)^{4}$ $\left(e_{0}^{\leftarrow}\right)^{2}\left(e_{1}^{\leftarrow}\right)^{3}\left(e_{2}^{\leftarrow}\right)^{4}\left(e_{3}^{\leftarrow}\right)^{4} e_{1}^{\leftarrow} e_{2}^{\leftarrow} e_{0}^{\leftarrow} e_{1}^{\leftarrow} e_{0}^{\leftarrow}$ and $R^{\uparrow}=e_{2}^{\uparrow}\left(e_{3}^{\uparrow}\right)^{2} e_{1}^{\uparrow}\left(e_{2}^{\uparrow}\right)^{3} e_{0}^{\uparrow}\left(e_{1}^{\uparrow}\right)^{3}\left(e_{0}^{\uparrow}\right)^{2}$.

Comparing (28) and (29), one may observe that in both cases the normal form $N$ is parametrised by the same partition $\lambda=(8,8,5,3,1)$. This can be explained using proposition 2.1, which implies that the same composite raising operations can be taken 


\subsection{Identifying the factors in the decomposition}

for $R^{\uparrow}$ in the binary case as for $R^{\leftarrow}$ in the integral case, up to the replacement of $e_{i}^{\uparrow}$ by $e_{i}^{\leftarrow}$; therefore the binary matrix $P$ and the integral matrix $Q$ both encode the same tableau $L$, of the shape $(9,8,5,5,3) /(4,1)$ of $M$. The raising operations in the given direction are exhausted, so this is a Littlewood-Richardson tableau, namely

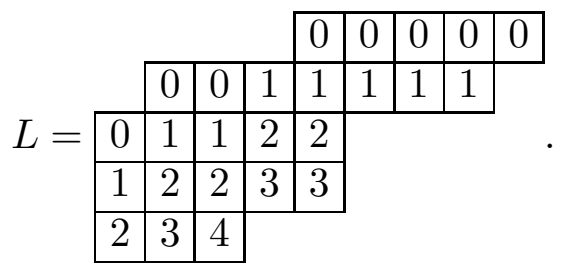

The partition $\operatorname{wt}(L)=(8,8,5,3,1)$ is equal to $\operatorname{row}(P)$ in the binary case and to $\operatorname{col}(Q)$ in the integral case, and in both cases it is therefore also equal to the partition $\lambda=$ $\operatorname{row}(N)$ that parametrises $N$. One can also read off $\lambda$ from the matrix $Q$ in the binary case, or from the matrix $P$ in the integral case, namely as $\lambda=\operatorname{col}(Q)^{t}$ respectively as $\lambda=\operatorname{row}(P)$. Those matrices are in fact the binary and integral encodings of the rectification $S$ of $T$, which has shape $\lambda$, since exhausting inward jeu de taquin slides applied to $T$ translates into particular sequences that exhaust leftward respectively upward crystal operations when applied to $M$, by proposition 2.5. That rectification is easily computed to be

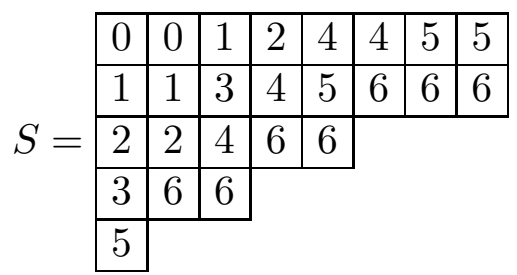

and its binary and integral encodings are indeed the binary matrix $Q$ above and the integral matrix $P$, respectively. The matrices $L$ and $S$ are the ones associated to $T$ under Robinson's bijection, as is described in [vLee3, corollary 3.3.4]; in the notation of that reference one has $(L, S)=\mathcal{R}(T)$.

Thus in a certain sense both bijections of theorem 3.1.3 model Robinson's bijection. However that point of view depends on interpreting matrices as encodings of tableaux, which might not always be natural. In any case one should be aware that one matrix can simultaneously encode tableaux of many different shapes. This point of view also gives somewhat incomplete information about double crystals, because even though the exhaustion of appropriate raising operations can be realised using jeu de taquin, it is not easy to do the same with individual raising operations.

\subsection{Identifying the factors in the decomposition.}

Since any matrix can easily be seen to encode a skew semistandard tableau of an appropriately chosen shape, the relation established above gives a complete characterisation of the bijections in theorem 3.1.3 that is independent of crystal operations. However, it describes the matrices $P$ and $Q$ in rather different terms, which seems unnatural given the characterisation of theorem itself. As we have observed above, the theorem in fact 
suggests a close relationship with the well known RSK correspondence (and its dual), in which the two straight tableaux associated to a matrix play a much more comparable role. We shall now proceed to characterise the bijections of our theorem in the language of those correspondences.

It is well known that the "P-tableau" of the pair that is associated under the RSK correspondence to an integral matrix $M$ can be computed using rectification by jeu de taquin; since the matrix $P$ of 3.1.3(2) can also be determined using rectification, this provides a useful starting point to relate the two correspondences. In more detail, the mentioned $P$-tableau can be found by rectifying a semistandard tableau $H$ whose shape is a horizontal strip, and whose "rows", taken from left to right, successively contain words (each of which is necessarily weakly increasing) whose weights given by the rows of $M$, from top to bottom. This observation was first made, for the case of Schensted correspondence, in [Schü2], see also [Stan, A1.2.6]; the generalisation to the RSK correspondence follows directly from its definition. For instance, the initial skew tableau corresponding to the matrix

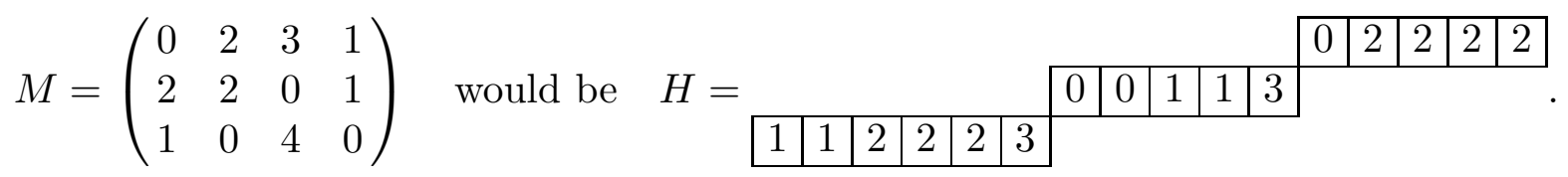

Note that by filling the horizontal strip from left to right, the vertical order of rows is interchanged: the topmost row of $M$ determines the bottom row of $H$. Therefore the relation between $M$ and $H$ is not that of a matrix encoding a tableau; rather the integral encoding of $H$ is the matrix formed by reversing the order of the rows of $M$. As for the matrix $P$ of 3.1.3(2), we have seen above that it is the integral encoding of the rectification $S$ of any tableau $T$ of which $M$ is the integral encoding. There are many possibilities for $T$, all of which differ only by horizontal shifts of their rows and which therefore have the same rectification. It is in particular always possible to take for $T$ a tableau whose shape is a horizontal strip. Comparing with what we said for the RSK correspondence, and using the fact that both for that correspondence and for the one of theorem 3.1.3(2), transposing $M$ results in interchanging $P$ and $Q$ (or actually $P$ and $Q^{t}$ for our theorem), we arrive at the following description.

3.3.1. Proposition. Let $M \in \mathcal{M}$, which we view as a some sufficiently large finite rectangular matrix. The corresponding matrices $P, Q \in \mathcal{M}$ of theorem 3.1.3(2) can be found as follows: $P$ is the integral encoding of the $P$-symbol under the RSK correspondence of the matrix obtained from $M$ by reversing the order of its rows, and $Q^{t}$ is the integral encoding of the $Q$-symbol under the RSK correspondence of the matrix obtained from $M$ by reversing the order of its columns.

Note that reversal of the order of rows or columns is not a well defined operation on $\mathcal{M}$, but the resulting ambiguity about the matrices of which the $P$-symbol and the $Q$-symbol are to be taken is harmless, since it only affects the other symbol associated to each matrix, the one that is not used in the proposition. For instance the matrix obtained by the reversal of the order of rows is only determined up to a vertical shift, but this only induces a shift in the entries of the associated $Q$-symbol, which is unused. 


\subsection{Identifying the factors in the decomposition}

The description of the proposition can be reformulated as follows: $P$ and $Q^{t}$ are the integral encodings of the insertion tableau for the traversal of $M$ in reverse Semitic reading order (by rows, read from left to right, ordered from bottom to top), rowinserting at each entry $M_{i, j}$ copies of the number $j$, respectively of the recording tableau for a similar insertion, but of negated values and traversing $M$ in the opposite (Semitic) order. The latter tableau can also be described either as the Schützenberger dual of the recording tableau for the initial insertion process, or as the insertion tableau for a traversal of $M$ in Kanji reading order, where at each entry $M_{i, j}$ copies of the number $i$ are row-inserted, but in all these cases this involves two separate applications of an RSK or Schützenberger algorithm.

However the pair of tableaux of which $P$ and $Q^{t}$ are encodings is in fact the pair associated to $M$ under the "Burge correspondence". This is a variation of the RSK correspondence that was first defined (for a somewhat specialised case) in [Bur], and which is discussed in detail in [Fult, A.4.1] and in [vLee4, §3.2]. To be more specific, this gives us the following description.

3.3.2. Proposition. The matrices $P, Q \in \mathcal{M}$ corresponding to $M \in \mathcal{M}$ under the bijection of theorem 3.1.3(2) are respectively the integral encoding of the $P$-symbol and the transpose of the integral encoding of the $Q$-symbol that are associated to $M$ under the Burge correspondence. This $P$-symbol can be obtained by traversing $M$ in the Semitic reading order (by rows from right to left) and successively column inserting, into an initially empty tableau, for each position $(i, j)$ encountered $M_{i, j}$ copies of the number $j$; the $Q$-symbol is the recording tableau $\left(\lambda^{(i)}\right)_{i \in \mathbf{N}}$ for this procedure, where $\lambda^{(i)}$ is the shape of the tableau under construction before the traversal of row $i$ of $M$.

The sequence of numbers that is being inserted is also the Semitic reading of any tableau of which $M$ is the integral encoding; for instance for the matrix $M$ in (29) this is the sequence $5,6,6 ; 2,3,4,6,6 ; 1,1,2,4,5 ; 0,1,3,4,6,6,6 ; 0,2,4,5,5$, read from right to left. (For column insertion it is best to imagine the entries being inserted as coming from the right, so writing this sequence backwards is actually quite natural; for instance, in the notation of [Fult] the insertion tableau may be written as $5 \rightarrow(6 \rightarrow(6 \rightarrow) \rightarrow$ $\cdots \rightarrow(2 \rightarrow(4 \rightarrow(5 \rightarrow(5)))) \cdots)))$, where we added redundant parentheses to stress the right-associativity of the operator ' $\rightarrow$ '.) Since column insertion can be simulated by jeu de taquin just like row insertion can, with as only difference that the entries inserted are initially arranged into a tableau according to the Semitic reading order, it is clear that the tableau obtained by column inserting the sequence given is equal to the rectification $S$ of the tableau $L$ given in (31). This argument justifies the "P"-part of the proposition above; to justify its " $Q$ "-part, the easiest argument is to recall that the Burge correspondence enjoys the same symmetry property as the RSK correspondence, and that the bijection of theorem 3.1.3(2) has a similar symmetry.

We illustrate this computation for the integral matrix $M$ of (29). We are performing column insertion, and the display below should be read from right to left; it shows the state of the insertion process after each row of $M$ is processed. The entries being inserted are written above the arrows between the tableaux; they too should be read from right to left to get their order of insertion. 
Indeed the final tableau coincides with $S$ of (31) that is encoded by $P$ of (29), and the sequence of shapes $(0)-(5)-(7,5)-(8,7,2)-(8,8,4,2)-(8,8,5,3,1)$ defines the semistandard Young tableau

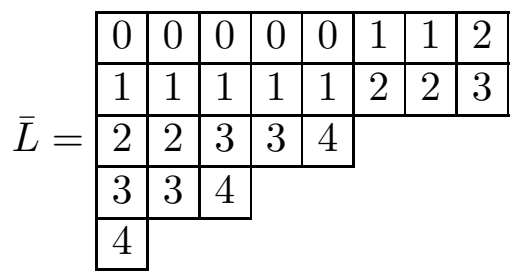

encoded by $Q^{t}$; it is the companion Young tableau of the Littlewood-Richardson tableau $L$ of (30).

For the bijection of 3.1.3(1), one can use a similar argument to identify the matrix $Q$, which is the binary encoding of the rectification of any tableau $T$ encoded by the binary tableau $M$. There always exists such a tableau $T$ whose shape is a vertical strip, and as in the case of a horizontal strip, the rectification can then be computed as the insertion tableau either for the column insertion of the entries taken from top right to bottom left, of for the row insertion of the entries taken in the opposite order. In either case the shape of the tableau grows by a vertical strip after the insertion of the entries for each complete column of $T$, which defines a transpose semistandard recording tableau; since its vertical strips are most naturally indexed by the numbers of the columns of $T$ traversed, the recording tableau for the right-to-left column insertion is actually a reverse tableau. As we have observed above, the binary matrix $P$ of 3.1.3(1) corresponds to a reverse transpose semistandard tableau, and this is in fact the recording tableau of the top right to bottom left column insertion of the entries of $T$ (for the row insertion of the entries in the opposite order, it is the Schützenberger dual of the recording tableau). One thus has the following description of the bijection between $M$ and $(P, Q)$ of 3.1.3(1).

3.3.3. Proposition. The matrices $P, Q \in \mathcal{M}^{[2]}$ corresponding to $M \in \mathcal{M}^{[2]}$ under the bijection of theorem 3.1.3(1) can be computed as follows. The matrix $Q$ is the binary encoding of the semistandard Young tableau $S$ obtained by traversing the matrix $M$ in the Kanji reading order (down columns, from right to left), and successively column inserting, into an initially empty tableau, for each bit $M_{i, j}$ equal to 1 an entry $i$. The matrix $P$ determines the reverse transpose semistandard Young tableau $R=\left(\lambda^{(j)}\right)_{j \in \mathbf{N}}$ that is the recording tableau for this insertion process: $\lambda^{(j)}=\sum_{l \geq j} P_{l}^{t}$ is the shape of the tableau under construction after the the traversal of column $j$ of $M$.

We illustrate this computation for the binary matrix $M$ of our running example. As before we display successive stages of column insertion from right to left; the strictly 


\subsection{Relation with decomposition of pictures}

increasing sequence of numbers inserted at each rightward arrow is written from top to bottom, as it is a column of the tableau $T$ of $(27)$.

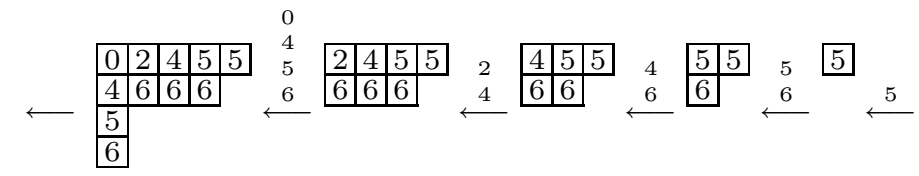

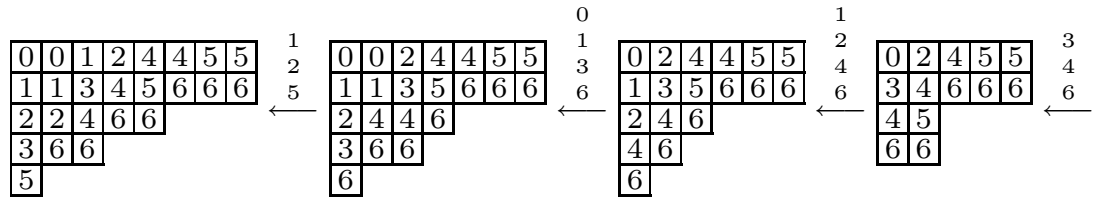

Indeed the final tableau coincides with the tableau $S$ of (31) of which the matrix $Q$ of (28) is the binary encoding, and the reverse transpose semistandard Young tableau $R$ given by the sequence of shapes $(8,8,5,3,1) \rightarrow(7,7,4,3,1) \rightarrow(6,6,3,2,1) \rightarrow(5,5,2,2) \rightarrow$ $(5,4,1,1) \rightarrow(4,3) \rightarrow(3,2) \rightarrow(2,1) \rightarrow(1) \rightarrow(0)$ corresponds to the binary matrix $P$ of (28). This tableau can be displayed by filling for each $i$ the squares of the vertical strip $\left[\lambda^{(i)} / \lambda^{(i+1)}\right]$ with entries $i$, giving

$$
R=\begin{array}{|l|l|l|l|l|l|l|l|}
\hline 8 & 7 & 6 & 5 & 4 & 2 & 1 & 0 \\
\hline 7 & 6 & 5 & 4 & 3 & 2 & 1 & 0 \\
\hline 4 & 3 & 2 & 1 & 0 & \multicolumn{7}{|l}{} \\
\hline 4 & 3 & 1 &
\end{array}
$$

We shall finally compare the bijection of 3.1.3(1) with the dual RSK correspondence. One should first of all realise that, unlike for the ordinary RSK and Burge correspondences, there is no universal agreement of which pair of tableaux corresponds to a given binary matrix (versions differ by transposition of one or both tableaux). We shall use the form in [Stan], where the entries coming from a conventional reading of $M$ are row-inserted into a transpose semistandard Young tableau, and the recording tableau is semistandard. Note that the following statement directly relates to the tableaux coming from the dual RSK correspondence applied to $M$ (even if a Schützenberger dual is involved), whereas for the integer correspondence we could not use the tableaux corresponding to $M$ itself under the RSK correspondence.

3.3.4. Proposition. The matrices $P, Q \in \mathcal{M}^{[2]}$ corresponding to $M \in \mathcal{M}^{[2]}$ under the bijection of theorem 3.1.3(1) are related as follows to the pair $\left(R^{*}, S\right)$ that is associated to $M$ under the dual RSK correspondence as described in [Stan, theorem 7.14.2]: the transpose semistandard Young tableau $R^{*}$ is the Schützenberger dual of the reverse transpose semistandard Young tableau $R$ determined by $P$, and $S$ is the semistandard Young tableau encoded by $Q$.

\subsection{Relation with decomposition of pictures.}

We have established relations between the bijections of theorem 3.1.3 and known correspondences defined by various insertion procedures, but the descriptions could use 
some clarification. We have justified the correspondences with insertion tableaux via their relation to jeu de taquin, but the correspondences with the recording tableau are only justified using symmetry properties for the integral case, and only by an example in the binary case. We shall now present a correspondence that explains both factors in a more symmetric way, and allows to transparently justify the descriptions of the previous subsection.

If an integral matrix $M$ satisfies $M \in \operatorname{Tabl}(\lambda / \kappa)$, it encodes a semistandard tableau $T$ of shape $\lambda / \kappa$, and if it also satisfies $M \in \operatorname{LR}(\nu / \mu)$, then $M^{t}$ encodes a semistandard tableau $\bar{T}$ of shape $\nu / \mu$, which is a companion tableau of $T$. The pair $(T, \bar{T})$ can be represented in a symmetric manner by the notion of a picture $\lambda / \kappa \rightarrow \nu / \mu$, which designates a particular kind of bijection between skew diagrams $[\lambda / \kappa] \rightarrow[\nu / \mu]$, in a way that we shall now describe.

In fact two different flavours of pictures can be defined, both of which have the property that the set of pictures $\lambda / \kappa \rightarrow \nu / \mu$ is in bijection with $\operatorname{Tabl}(\lambda / \kappa) \cap \operatorname{LR}(\nu / \mu)$, and both of which give rise to essentially the same theory. Our reference for the theory of pictures will be [vLee2], but we shall define the opposite flavour of pictures than was used there, since that simplifies the correspondences that are of interest here. This means that we must adapt the results we cite to our situation; in fact the form of these results changes little between the two flavours of pictures, except for explicit references to the Schensted row insertion procedure (for instance in theorem 3.2.1 and lemma 3.3 .2 of [vLee2]), which must be replaced by column insertion. The pictures used in the present paper are defined as follows (the difference with the other flavour of pictures is the use of ' $\leq$,' rather than its opposite partial ordering).

\subsubsection{Definitions.}

a. The partial orderings ' $\leq_{\nwarrow}$ ' and ' $\leq_{\nearrow}$ ' on $\mathbf{N}^{2}$ are defined by $(i, j) \leq_{\nwarrow}(k, l)$ if and only if $i \leq k$ and $j \leq l$, respectively by $(i, j) \leq,(k, l)$ if and only if $i \leq k$ and $j \geq l$.

b. For skew shapes $\lambda / \kappa$ and $\nu / \mu$, a picture $\lambda / \kappa \rightarrow \nu / \mu$ is a bijection $f:[\lambda / \kappa] \rightarrow[\nu / \mu]$ between their diagrams, such that $s \leq_{\nwarrow} t$ implies $f(s) \leq_{\nearrow} f(t)$ and $f(s) \leq_{\nwarrow} f(t)$ implies $s \leq, t$ for all $s, t \in[\lambda / \kappa]$. The set of pictures $\lambda / \kappa \rightarrow \nu / \mu$ is denoted by $\operatorname{Pic}(\lambda / \kappa, \nu / \mu)$.

c. For a picture $f \in \operatorname{Pic}(\lambda / \kappa, \nu / \mu)$, the matrices $\operatorname{Int}(f) \in \mathcal{M}_{\lambda-\kappa, \nu-\mu}$ and $\operatorname{Bin}(f) \in$ $\mathcal{M}_{\nu-\mu, \lambda^{t}-\kappa^{t}}^{[2]}$ are respectively given by

$$
\operatorname{Int}(f)_{i, k}=\#\left\{(j, l) \in \mathbf{N}^{2} \mid(i, j) \in[\lambda / \kappa],(k, l) \in[\nu / \mu], f(i, j)=(k, l)\right\}
$$

and by

$$
\operatorname{Bin}(f)_{k, j}=\#\left\{(i, l) \in \mathbf{N}^{2} \mid(i, j) \in[\lambda / \kappa],(k, l) \in[\nu / \mu], f(i, j)=(k, l)\right\} .
$$

The expressions in part c can be described in words as follows: $\operatorname{Int}(f)_{i, k}$ counts the number of squares of row $i$ that the picture $f$ maps to row $k$, while $\operatorname{Bin}(f)_{k, j}$ counts the number of squares of column $j$ that $f$ maps to row $k$.

From the form of part $\mathrm{b}$ it is clear that the inverse of a picture $\lambda / \kappa \rightarrow \nu / \mu$ is a picture $\nu / \mu \rightarrow \lambda / \kappa$. The point of part $\mathrm{c}$ of the definition is the following fact. 


\subsection{Relation with decomposition of pictures}

3.4.2. Proposition. For any pair of skew shapes $\lambda / \kappa$ and $\nu / \mu$, the operations Int and $\operatorname{Bin}$ define bijections from $\operatorname{Pic}(\lambda / \kappa, \nu / \mu)$ respectively to $\operatorname{Tabl}(\lambda / \kappa) \cap \operatorname{LR}(\nu / \mu)$ and to $\operatorname{Tabl}^{[2]}(\lambda / \kappa) \cap \operatorname{LR}^{[2]}(\nu / \mu)$.

Proof. For a given matrix $M$, a picture $f$ with $\operatorname{Int}(f)=M$ or $\operatorname{Bin}(f)=M$ is straightforwardly constructed, defining images of the squares of $[\lambda / \kappa]$ in the Semitic (in the integral case) or the Kanji (in the binary case) order. The conditions of 3.4.1b allow only one candidate image for each square, and one checks that the tableau and Littlewood-Richardson conditions for $M$ assure that this is a valid choice.

When $\nu / \mu$ is a horizontal strip, the conditions $M \in \operatorname{LR}(\nu / \mu)$ and $M \in \operatorname{LR}^{[2]}(\nu / \mu)$ just require $\operatorname{col}(M)=\nu-\mu$ respectively $\operatorname{row}(M)=\nu-\mu$, so in this case $\operatorname{Pic}(\lambda / \kappa, \nu / \mu)$ is in bijection with the set of tableaux in $\operatorname{SST}(\lambda / \kappa)$ of weight $\nu-\mu$. Moreover, if $f$ so corresponds to a tableau $T$, then $\operatorname{Int}(f)$ and $\operatorname{Bin}(f)$ are respectively the integral and binary encodings of $T$. Thus we find our encoding of semistandard tableaux by integral or binary matrices as a special case of representing pictures by matrices using Int $(f)$ or $\operatorname{Bin}(f)$. Similarly, if $\nu / \mu$ is arbitrary but $\lambda / \kappa$ is a horizontal strip, then the pictures in $\operatorname{Pic}(\lambda / \kappa, \nu / \mu)$ are in bijection, via their inverse picture, with tableaux in $\operatorname{SST}(\nu / \mu)$ of weight $\lambda-\kappa$. In the latter case $\operatorname{Int}(f)=\operatorname{Int}\left(f^{-1}\right)^{t} \in \operatorname{LR}(\nu / \mu)$ is the transpose of the integral encoding of the tableau thus corresponding to $f$; however there is in this case no similar interpretation for $\operatorname{Bin}(f)$ (which is in fact a rather sparse matrix, having column sums of at most 1 ). On the other hand, if $\lambda / \kappa$ is a vertical strip rather than a horizontal one, then it is most practical to consider $\operatorname{Bin}(f)$ instead of $\operatorname{Int}(f)$ : in this case $\operatorname{Pic}(\lambda / \kappa, \nu / \mu)$ is in bijection with the set of reverse transpose semistandard tableaux of shape $\nu / \mu$ and weight $\lambda^{t}-\kappa^{t}$, and their correspondence to the matrix $\operatorname{Bin}(f) \in \operatorname{LR}^{[2]}(\nu / \mu)$ is exactly the one that we used before.

A central result concerning pictures is the decomposition theorem [vLee2, theorem 3.2.1], which generalises the Robinson-Schensted and RSK correspondences; it gives a bijection between $\operatorname{Pic}(\lambda / \kappa, \nu / \mu)$ and the union over all $\pi \in \mathcal{P}$ of Cartesian products $\operatorname{Pic}(\pi, \nu / \mu) \times \operatorname{Pic}(\lambda / \kappa, \pi)$. This bijection can be defined by a version of the Schensted algorithm using (for our notion of pictures) column insertion, translated into the language of pictures, as described in [vLee2, lemma 3.3.2]. It follows that for specific shapes $\lambda / \kappa$ and $\nu / \mu$, this decomposition specialises under the appropriate correspondences to the column insertion Robinson-Schensted correspondence, to the Burge correspondence, and to the version of the dual RSK correspondence using column insertion described in our proposition 3.3.3. For the Robinson-Schensted correspondence one takes $\lambda / \kappa$ and $\nu / \mu$ to be shapes that are both horizontal and vertical strips, so that $\operatorname{Pic}(\lambda / \kappa, \nu / \mu)$ consists of all bijections $[\lambda / \kappa] \rightarrow[\nu / \mu]$, and that $\operatorname{Pic}(\pi, \nu / \mu)$ and $\operatorname{Pic}(\lambda / \kappa, \pi)$ are both in bijection with the set of standard Young tableaux of shape $\pi$. For the other two cases, one takes $\nu / \mu$ to be a horizontal strip, and $\lambda / \kappa$ to be a horizontal respectively a vertical strip. Then the operations Int and $\operatorname{Bin}$ define bijections $\operatorname{Pic}(\lambda / \kappa, \nu / \mu) \rightarrow \mathcal{M}_{\lambda-\kappa, \nu-\mu}$ respectively $\operatorname{Pic}(\lambda / \kappa, \nu / \mu) \rightarrow \mathcal{M}_{\nu-\mu, \lambda^{t}-\kappa^{t}}^{[2]}$, and the sets $\operatorname{Pic}(\pi, \nu / \mu)$ and $\operatorname{Pic}(\lambda / \kappa, \pi)$ are in bijection with sets of semistandard Young tableaux of shape $\pi$ as described above. Thus these latter two special cases of the decomposition of pictures give rise, under application of Bin and Int, precisely to the bijections that occur in our theorem 3.1.3. 
The description of the decomposition of pictures using the column insertion Schensted algorithm is rather different from the definition of our bijections in theorem 3.1.3. However, the decomposition of pictures has an alternative description that is given in [vLee2, theorem 5.4.1]. This description is in terms of two forms of jeu de taquin that are defined for pictures, one that changes the shape $\lambda / \kappa$ of the domain, and another that changes the shape $\nu / \mu$ of the image of the picture. The two types of operations commute [vLee2, theorem 5.3.1], and a decomposition of pictures based on these operations follows in a manner quite similar to the way we obtained theorem 3.1.3. From the fact that for pictures Schensted column insertion can be emulated by jeu de taquin (as it can for tableaux), it follows that the two descriptions define the same decomposition of pictures. Under the operation Int, each domain slide of a picture corresponds to a sequence of upward crystal operations on integer matrices, just like in proposition 2.5, and each image slide corresponds to a sequence of leftward crystal operations; hence the decomposition of pictures translates directly into the decomposition of integral matrices of theorem 3.1.3(2). For the operation Bin, the situation is similar, except that domain slides correspond to leftward crystal operations and image slides correspond to upward crystal operations. Thus the decomposition of pictures also translates into the decomposition of binary matrices of theorem 3.1.3(2).

With these links between the decomposition of matrices and that of pictures, and the fact that the latter can be computed using Schensted column insertion, the descriptions of the previous subsection that involve column insertion are easy to understand. For instance, the facts that in proposition 3.3.2 the integral matrix $M$ is traversed in Semitic order while in proposition 3.3.3 the binary matrix $M$ is traversed in Kanji ordering both reflect the fact that the column insertions used to compute the first factor of the decomposition of a picture traverse its domain from top-right to bottom-left (in fact any order will do that never makes a decreasing step for ' $\leq{ }_{\nearrow}^{\prime}$ '). This is so because $\mathcal{M}_{\alpha, \beta}$ is modelled by $\operatorname{Pic}(\lambda / \kappa, \nu / \mu)$ where $\lambda / \kappa$ and $\nu / \mu$ are horizontal strips with $\lambda-\kappa=\alpha$ and $\nu-\mu=\beta$, and with the rows of the matrix corresponding to those of $\lambda / \kappa$, while $\mathcal{M}_{\alpha, \beta}^{[2]}$ is modelled by $\operatorname{Pic}(\lambda / \kappa, \nu / \mu)$ where $\lambda / \kappa$ is a vertical strip and $\nu / \mu$ a horizontal strip, with $\lambda^{t}-\kappa^{t}=\beta$ and $\nu-\mu=\alpha$, and with the columns of the matrix corresponding to those of $\lambda / \kappa$. In both cases top-right to bottom-left traversal of $[\lambda / \kappa]$ corresponds to the indicated traversal of the matrix. The description of the recording tableaux is also a direct translation of the way recording tableaux are defined for pictures. For propositions 3.3.1 and 3.3.4, which use row insertion, the more complicated formulations reflect a less direct correspondence to the decomposition of pictures; they can however be understood by using the symmetry properties of pictures that are discussed in [vLee $2, \S 4]$; we shall not elaborate the details here. 


\section{$\S 4$. Growth diagrams.}

After what was said in the last subsection, one might be tempted to conclude that crystal operations on matrices and the correspondences of theorem 3.1.3 are merely shadows of similar structures for pictures under the maps 'Int' and 'Bin', and that as such they do not add much insight, although they are somewhat simpler to present than pictures and the operations of jeu de taquin on them. In this section we shall however establish a link with the descriptions as those in [Roby] and [Fom] of Knuth correspondences using growth diagrams, which link does not exist in the theory of pictures. In doing so we build a bridge between the previously unrelated approaches to Knuth correspondences via jeu de taquin on one hand, and via growth diagrams on the other hand (the approach via jeu de taquin often also uses invariants, an in [Schü2] or [Fult]; we shall discuss these below in $\S 5)$.

\subsection{Submatrices and implicit shapes.}

For a discussion of the notion of growth diagrams we refer to [vLee4, $\S \S 2,3]$. In general, a growth diagram associates "shapes" to the elements of the grid $\mathbf{N} \times \mathbf{N}$, and it also associates "entries" to squares of the grid; in our case the shapes will be partitions, and the entries are our usual matrix entries, lying either in $[2]=\{0,1\}$ or in $\mathbf{N}$. Formally a square of the grid is an element of $\mathbf{N} \times \mathbf{N}$, just like a grid point is, but the "square" $(i, j)$ is supposed to have as corners the "grid points" in $\{i, i+1\} \times\{j, j+1\}$. We shall build a growth diagram from a given matrix $M$ by associating to the grid point $(k, l)$ a shape determined by the submatrix $\left(M_{i, j}\right)_{i \in[k], j \in[l]}$, of which that grid point is the lower right hand corner. The shape will in fact be the one that parametrises the normal form of that submatrix, which by our usual interpretation of finite matrices is identified with the matrix obtained from $M$ by clearing the entries outside $[k] \times[l]$; we shall call this the implicit shape of the submatrix. The formal definitions are as follows.

4.1.1. Definitions. Let $M \in \mathcal{M}^{[2]}$ or $M \in \mathcal{M}$, and let $N$ be the normal form of $M$, obtainable from it by exhausting the operations chosen from $\left\{e_{i}^{\uparrow} \mid i \in \mathbf{N}\right\} \cup\left\{e_{j}^{\leftarrow} \mid j \in \mathbf{N}\right\}$. We define

a. $\pi(M)=\operatorname{row}(N)$, which we call the implicit shape of $M$;

b. $M_{I \times J}=\left([(i, j) \in I \times J] M_{i, j}\right)_{i, j \in \mathbf{N}}$ for intervals $I, J \subseteq \mathbf{N}$, which is the matrix of the same type (binary or integral) as $M$ and obtained from if by replacing all entries $M_{i, j}$ with $(i, j) \notin I \times J$ by 0 .

The term implicit shape refers to the fact that it cannot be easily read off directly from $M$, although in $\S 5$ we shall give a characterisation that does not require modifying $M$. Once either of the factors $P, Q$ associated to $M$ under the decomposition of theorem 3.1.3 has been determined, the implicit shape of $M$ can however be read off, for instance as $\pi(M)=$ row $(P)$; in other words it is the shape of the tableaux corresponding to $M$ under the Burge correspondence or the column insertion dual RSK correspondence. If $M$ is the encoding of a skew semistandard tableau $T$, then $\pi(M)$ is the shape of the rectification of $T$.

Growth diagrams should satisfy a local rule, which we called "shape datum" in [vLee4], that relates the shapes at the four corners of any grid square $(i, j)$ and 
the matrix entry in that square. This rule is such that when the shapes at $(i+1, j)$ and $(i, j+1)$ are arbitrarily fixed, it defines a bijection between the possible values for the pair consisting of the shape at $(i, j)$ and the matrix entry on one hand, and the possible values for the shape at $(i+1, j+1)$ on the other hand. When the shape datum is prescribed, this permits deducing the entire growth diagram from a partial specification of it, namely from the shapes along any lattice path in the grid from bottom left to top right, together with all matrix entries to the bottom right of that path. In our current situation this will eventually provide alternative methods to compute implicit shapes, but at this point we just have the matrix of shapes $\left(\pi\left(M_{[i] \times[j]}\right)\right)_{i, j \in \mathbf{N}}$, which is defined without any reference to a shape datum. So we must first prove that it indeed defines a growth diagram, and determine the shape datum for which it does so.

\subsection{Growth diagrams for integral matrices.}

The key to proving that $\left(\pi\left(M_{[i] \times[j]}\right)\right)_{i, j \in \mathbf{N}}$ defines a growth diagram will be the study of a particular way of reducing $M$ to its normal form. Consider for the moment integral matrices, and take the matrix $M$ of (29). We start by exhausting the raising operations in $\left\{e_{i}^{\uparrow} \mid i \in[2]\right\}$ and then exhaust those in $\left\{e_{j}^{\leftarrow} \mid j \in[5]\right\}$; this gives the following transformations

$$
\left(\begin{array}{lllllll}
1 & 0 & 1 & 0 & 1 & 2 & 0 \\
1 & 1 & 0 & 1 & 1 & 0 & 3 \\
0 & 2 & 1 & 0 & 1 & 1 & 0 \\
0 & 0 & 1 & 1 & 1 & 0 & 2 \\
0 & 0 & 0 & 0 & 0 & 1 & 2
\end{array}\right) \stackrel{\left(e_{[2]}^{\uparrow}\right)^{\longrightarrow}}{\longrightarrow}\left(\begin{array}{ccccccc}
2 & 1 & 1 & 0 & 2 & 2 & 0 \\
0 & 2 & 0 & 1 & 0 & 1 & 3 \\
0 & 0 & 1 & 0 & 1 & 0 & 0 \\
\hline 0 & 0 & 1 & 1 & 1 & 0 & 2 \\
0 & 0 & 0 & 0 & 0 & 1 & 2
\end{array}\right) \stackrel{\left(e_{[5]}^{\leftarrow}\right) *}{\longrightarrow}\left(\begin{array}{llllll|l}
8 & 0 & 0 & 0 & 0 & 0 & 0 \\
0 & 4 & 0 & 0 & 0 & 0 & 3 \\
0 & 0 & 2 & 0 & 0 & 0 & 0 \\
\hline 0 & 1 & 1 & 1 & 0 & 0 & 2 \\
0 & 0 & 0 & 0 & 1 & 0 & 2
\end{array}\right)
$$

One sees that the submatrix indexed by [3] $\times[6]$ has been reduced to the normal form $\operatorname{Diag}((8,4,2))$. We shall first argue that this is indeed the normal form of the original submatrix $M_{[3] \times[6]}$, and that this reduction is no accident (a similar reduction can be achieved for any integral matrix).

4.2.1. Proposition. Fix $M \in \mathcal{M}$, and $k, l \in \mathbf{N}_{>0}$. Let $M^{\prime} \in \mathcal{M}$ be the matrix obtained from $M$ by exhausting the operations in $\left\{e_{i}^{\uparrow} \mid i \in[k-1]\right\} \cup\left\{e_{j}^{\leftarrow} \mid j \in[l-1]\right\}$, and let $N \in \mathcal{M}$ be the normal form of $M_{[k] \times[l]}$, which is obtained from it by exhausting the same set of crystal operations. Then $M_{[k] \times[l]}^{\prime}=N$.

Proof. It is clear that the normalisation of $M_{[k] \times[l]}$ cannot involve any raising operations other than the kind being applied to $M$, as $M_{[k] \times[l]}$ contains no units that could be transferred by such raising operations. It is also clear that the operations applied to $M$ cannot transfer units across the boundaries of the rectangle $[k] \times[l]$ : they either transfer units within that rectangle or within its complement. If we extract, from a sequence that exhausts these operations applied to $M$, those that involve a transfer within $[k] \times[l]$, then the extracted sequence can be applied to $M_{[k] \times[l]}$, and each operation will involve the same transfer as it did in the original sequence; this is so because any valid transfer in a matrix remains valid in any submatrix containing the squares involved in the transfer. Therefore the extracted sequence reduces $M_{[k] \times[l]}$ to $M_{[k] \times[l]}^{\prime}$, 


\subsection{Growth diagrams for integral matrices}

and it remains to show that the latter matrix is a normal from, which then necessarily equals $N$. This means that we have to prove that $n_{i}^{\uparrow}\left(M_{[k] \times[l]}^{\prime}\right)=n_{j}^{\leftarrow}\left(M_{[k] \times[l]}^{\prime}\right)=0$ for any $i \in[k-1]$ and $j \in[l-1]$, while we know that $n_{i}^{\uparrow}\left(M^{\prime}\right)=n_{j}^{\leftarrow}\left(M^{\prime}\right)=0$. Now if $U \mid V$ denotes an integral matrix vertically split into submatrices $U, V$, then it follows from (18) that $n_{i}^{\uparrow}(U \mid V) \geq n_{i}^{\uparrow}(U)$, so $n_{i}^{\uparrow}(U \mid V)=0$ implies $n_{i}^{\uparrow}(U)=0$. For a horizontal division into submatrices, (20) similarly gives $n_{j}^{\leftarrow}\left(\frac{S}{T}\right) \geq n_{j}^{\leftarrow}(S)$, so that $n_{j}^{\leftarrow}\left(\frac{S}{T}\right)=0$ implies $n_{j}^{\leftarrow}(S)=$ 0 . These relations allow us to conclude $n_{i}^{\uparrow}\left(M_{[k] \times[l]}^{\prime}\right)=n_{j}^{\leftarrow}\left(M_{[k] \times[l]}^{\prime}\right)=0$ as desired.

Note that while the final part of this argument amounts to showing that if any raising operation can be applied to a submatrix of the form $M_{[k] \times[l]}$, then the same operation could also be applied to the full matrix $M$, this does not mean that these operations would necessarily involve the same transfer. Indeed, if one has $n_{i}^{\uparrow}(U \mid V)>$ $n_{i}^{\uparrow}(U)>0$, then the first application(s) of $e_{i}^{\uparrow}$ to $U \mid V$ will be between entries of $V$, and only once $n_{i}^{\uparrow}(U \mid V)$ and $n_{i}^{\uparrow}(U)$ have become equal will the remaining applications of $e_{i}^{\uparrow}$ transfer entries within $U$. The same is true for leftward moves with respect to a horizontal split. In the example given one sees that the exhaustion of operations in $e_{[5]}^{\leftarrow}$ involves leftward transfers below as well as above the horizontal line; the former precede the latter in case of transfers between the same pair of columns.

Let us now consider the relation between the four shapes $\pi\left(M_{[i] \times[j]}\right)$ for $(i, j) \in$ $\{k, k+1\} \times\{l, l+1\}$ and the matrix entry $M_{k, l}$. All of these values only depend in the submatrix $M_{[k+1] \times[l+1]}$, and none of them changes when one applies operations in $\left\{e_{i}^{\uparrow} \mid i \in[k-1]\right\} \cup\left\{e_{j}^{\leftarrow} \mid j \in[l-1]\right\}$. Therefore let $A$ be the matrix obtained from $M_{[k+1] \times[l+1]}$ by exhausting these operations, and to simplify the notation put $\lambda=\pi\left(M_{[k] \times[l]}\right), \quad \mu=\pi\left(M_{[k] \times[l+1]}\right), \quad \nu=\pi\left(M_{[k+1] \times[l]}\right)$, and $\kappa=\pi\left(M_{[k+1] \times[l+1]}\right)$, which are also the implicit shapes of the corresponding submatrices of $A$ (here we abandon our previous conventions for the use of $\lambda, \mu, \nu, \kappa$, and adopt those of [vLee4]). By proposition 4.2.1 we have $A_{[k] \times[l]}=\operatorname{Diag}(\lambda)$.

The normalisation of $A_{[k+1] \times[l]}$ will only involve upward transfers, since the leftward operations in $\left\{e_{j}^{\leftarrow} \mid j \in[l-1]\right\}$ remain exhausted throughout. This normalisation transfers any units present in (the bottom) row $k$ of $A_{[k+1] \times[l]}$ upwards, until they arrive on the main diagonal, where together with the units that were already there in $A_{[k] \times[l]}=\operatorname{Diag}(\lambda)$ they form the matrix $\operatorname{Diag}(\nu)$. It follows that row $k$ of $A_{[k+1] \times[l]}$ must have been equal to the composition $\nu-\lambda$ (and in particular that it contained no units strictly above the main diagonal). A similar argument shows that the final column $l$ of $A_{[k] \times[l+1]}$ is equal to $\mu-\lambda$. The matrix $A$ is therefore completely determined by the values of $\lambda, \mu, \nu$, and $M_{k, l}$ : one has $A_{[k] \times[l]}=\operatorname{Diag}(\lambda), A_{i, l}=\mu_{i}-\lambda_{i}$ for $i \in[k]$, $A_{k, j}=\nu_{j}-\lambda_{j}$ for $j \in[l]$, and $A_{k, l}=M_{k, l}$. This establishes one part of requirement for families of shapes $\left(\pi\left(M_{[i] \times[j]}\right)\right)_{i, j \in \mathbf{N}}$ to define a growth diagram, namely that $\lambda, \mu, \nu$, and $M_{k, l}$ together determine $\kappa$; it also provides a procedure to compute this direction of the shape datum (namely construct the matrix $A$ and find its implicit shape). It is easy to see that one has $|\kappa|=|\mu|+|\nu|-|\lambda|+M_{i, j}$ as required by the definition of a shape datum [vLee4, definition 2.2.1].

Let us apply the procedure for computing $\kappa$ in the case of the matrix $M$ of (29) that was used in the example above, for $k=3$ and $l=6$; so our goal is to compute 
$\kappa=\pi\left(M_{[4] \times[7]}\right)$. From the last matrix in (36) one reads off $\lambda=(8,4,2), \mu-\lambda=(0,3,0)$ so that $\mu=(8,7,2), \quad \nu-\lambda=(0,1,1,1,0,0)$ so that $\nu=(8,5,3,1)$, and $M_{3,6}=2$. We may now determine the following diagram, from which it can be read off that $\kappa=(8,8,4,2)$ (unlike the previously drawn diagrams we now let the arrows between the matrices point in the direction opposite to the crystal operations that are being applied; this is done so that the rectangle $[i] \times[j]$ for which the submatrix has been normalised to $\pi\left(A_{[i] \times[j]}\right)$ grows from left to right and from top to bottom).

$$
\begin{aligned}
& A=\left(\begin{array}{cccccc|c}
8 & 0 & 0 & 0 & 0 & 0 & 0 \\
0 & 4 & 0 & 0 & 0 & 0 & 3 \\
0 & 0 & 2 & 0 & 0 & 0 & 0 \\
\hline 0 & 1 & 1 & 1 & 0 & 0 & 2
\end{array}\right) \quad \stackrel{\left(e_{[6]}^{\leftarrow}\right)^{*}}{\longrightarrow}\left(\begin{array}{ccccccc}
8 & 0 & 0 & 0 & 0 & 0 & 0 \\
0 & 7 & 0 & 0 & 0 & 0 & 0 \\
0 & 0 & 2 & 0 & 0 & 0 & 0 \\
\hline 0 & 1 & 2 & 2 & 0 & 0 & 0
\end{array}\right) \\
& \left(e_{[3]}^{\uparrow}\right)^{*} \downarrow \quad\left(e_{[3]}^{\uparrow}\right)^{*} \downarrow \\
& \left(\begin{array}{llllll|l}
8 & 0 & 0 & 0 & 0 & 0 & 0 \\
0 & 5 & 0 & 0 & 0 & 0 & 3 \\
0 & 0 & 3 & 0 & 0 & 0 & 1 \\
0 & 0 & 0 & 1 & 0 & 0 & 1
\end{array}\right) \stackrel{\left(e_{[6]}^{\leftarrow}\right)^{*}}{\longrightarrow}\left(\begin{array}{ccccccc}
8 & 0 & 0 & 0 & 0 & 0 & 0 \\
0 & 8 & 0 & 0 & 0 & 0 & 0 \\
0 & 0 & 4 & 0 & 0 & 0 & 0 \\
0 & 0 & 0 & 2 & 0 & 0 & 0
\end{array}\right)=\operatorname{Diag}(\kappa)
\end{aligned}
$$

To complete our argument we shall also have to show that conversely $\mu, \nu$, and $\kappa$ determine $\lambda$ and $M_{k, l}$, but before we do so we must specify which relations should hold among the shapes for the above construction to apply. First of all, each implicit shape $\pi\left(M_{[i] \times[j]}\right)$ can have at most $\min (i, j)$ nonzero parts. Apart from that and $\lambda \subseteq \mu$ and $\lambda \subseteq \nu$ (which must hold since neither $\nu-\lambda$ nor $\mu-\lambda$ have negative entries), there is only one requirement for $\lambda, \mu$, and $\nu$, namely that the matrix $A$ constructed from the pieces $\operatorname{Diag}(\lambda), \mu-\lambda, \mu-\lambda$ and $M_{k, l}$ as indicated must satisfy the hypothesis of initial exhaustion, in other words that $n_{i}^{\uparrow}(A)=n_{j}^{\leftarrow}(A)=0$ for all $i \in[k-1]$ and $j \in[l-1]$. This amounts to the requirement $\lambda_{i+1}-\lambda_{i}+(\mu-\lambda)_{i+1} \leq 0$ or equivalently $\mu_{i+1} \leq \lambda_{i}$ for $i \in[k-1]$, and similarly $\nu_{j+1} \leq \lambda_{j}$ for $j \in[l-1]$. Together with the limit on the number of nonzero parts, this implies that $\lambda, \mu$, and $\nu$ satisfy the relations $\lambda-\mu$ and $\lambda \leftarrow \nu$. Conversely, for any $\lambda, \mu, \nu \in \mathcal{P}$ satisfying $\mu_{\min (k, l+1)}=\nu_{\min (k+1, l)}=0$, and the relations $\lambda-\mu$, and $\lambda-\nu$, the construction of $A$ (with an arbitrary entry $A_{k, l} \in \mathbf{N}$ ) provides an example where $\lambda, \mu$, and $\nu$ occur as the implicit shapes of appropriate submatrices.

Now assume that $\mu, \nu, \kappa \in \mathcal{P}$ are given with $\mu_{\min (k, l+1)}=\nu_{\min (k+1, l)}=0, \mu-\kappa$, and $\nu \leftarrow \kappa$. We want to find $A$ as above, giving rise to these shapes. We know its normal form $\operatorname{Diag}(\kappa)$, as well as the normal forms $\operatorname{Diag}(\mu)$ and $\operatorname{Diag}(\nu)$ of $A_{[k] \times[l+1]}$ and $A_{[k+1] \times[l]}$, respectively. Exhausting the operations $\left\{e_{j}^{\leftarrow} \mid j \in[l]\right\}$ applied to $A$ must give the $(k+1) \times(l+1)$ matrix $B$ whose restriction to $[k] \times[l+1]$ equals $\operatorname{Diag}(\mu)$ and whose row $k$ equals $\kappa-\mu$. The normal form $\operatorname{Diag}(\kappa)$ of $B$ can be written as $R^{\uparrow}(B)$ for some sequence $R^{\uparrow}$ of upward crystal operations in $\left\{e_{i}^{\uparrow} \mid i \in[k]\right\}$. Then $R^{\uparrow}$ can also be applied to $A$, exhausting upward transfers and resulting in the $(k+1) \times(l+1)$ matrix $C$ whose restriction to $[k+1] \times[l]$ equals $\operatorname{Diag}(\mu)$ and whose column $l$ equals $\kappa-\nu$. Then inverting the operations in $R^{\uparrow}$, it is clear that $A=R^{\downarrow}(C)$, and from this matrix one can extract the values $\lambda=\pi\left(A_{[k] \times[l]}\right)$ and $M_{k, l}=A_{k, l}$. 


\subsection{Growth diagrams for integral matrices}

As an example of the computation in the inverse direction, let partitions $\kappa=$ $(13,9,9,5), \mu=(11,9,8)$, and $\nu=(10,9,8,2)$ be given, and take $k=3$ and $l=4$ (the minimal possible values, given $\mu$ and $\nu$ ). We form a commutative diagram like the one drawn above, but starting with the matrices $B, C$, and $\operatorname{Diag}(\kappa)$ below, which correspond to the initial data. One easily determines composite raising operations transforming $B$ or $C$ into $\operatorname{Diag}(\kappa)$, for instance $R^{\uparrow}=\left(e_{0}^{\uparrow}\right)^{2}\left(e_{1}^{\uparrow}\right)^{2}\left(e_{2}^{\uparrow}\right)^{3}$ and $R^{\leftarrow}=\left(e_{0}^{\leftarrow}\right)^{3}\left(e_{1}^{\leftarrow}\right)^{3}\left(e_{2}^{\leftarrow}\right)^{4}\left(e_{3}^{\leftarrow}\right)^{7}$ (the exponents are just initial partial sums of the parts of the compositions $\kappa-\mu=$ $(2,0,1,5)$ and $\kappa-\nu=(3,0,1,3))$. Then one computes $A$ by inverting one of these composite operations, for instance $A=R^{\downarrow}(C)$ where $R^{\downarrow}=\left(f_{2}^{\downarrow}\right)^{3}\left(f_{1}^{\downarrow}\right)^{2}\left(f_{0}^{\downarrow}\right)^{2}$, and from $A$ one then reads off $\lambda=(9,9,6)$ and $M_{3,4}=3$.

$$
\begin{aligned}
A & =\left(\begin{array}{cccc|c}
9 & 0 & 0 & 0 & 2 \\
0 & 9 & 0 & 0 & 0 \\
0 & 0 & 6 & 0 & 2 \\
\hline 1 & 0 & 2 & 2 & 3
\end{array}\right) \stackrel{R^{\leftarrow}}{\longrightarrow}\left(\begin{array}{ccccc}
11 & 0 & 0 & 0 & 0 \\
0 & 9 & 0 & 0 & 0 \\
0 & 0 & 8 & 0 & 0 \\
\hline 2 & 0 & 1 & 5 & 0
\end{array}\right)=B \\
C & =\left(\begin{array}{cccc|c}
10 & 0 & 0 & 0 & 3 \\
0 & 9 & 0 & 0 & 0 \\
0 & 0 & 8 & 0 & 1 \\
0 & 0 & 0 & 2 & 3
\end{array}\right) \stackrel{R^{\uparrow}}{\longrightarrow}\left(\begin{array}{ccccc}
13 & 0 & 0 & 0 & 0 \\
0 & 9 & 0 & 0 & 0 \\
0 & 0 & 9 & 0 & 0 \\
0 & 0 & 0 & 5 & 0
\end{array}\right)=\operatorname{Diag}(\kappa) .
\end{aligned}
$$

As is clear from the examples, the only aspect of the reduction $A \rightarrow \operatorname{Diag}(\kappa)$ that is not completely straightforward is the transformation during the first reduction phase of the row or column that is outside the submatrix being normalised in that phase. Consider for instance applying upward transfers to reduce the submatrix $A_{[k+1] \times[l]}$ to its normal form $\operatorname{Diag}(\nu)$. Here column $l$, which initially describes the composition $\alpha=\mu-\lambda$ and the entry $M_{k, l}$, is transformed into a composition $\alpha^{\prime}$ that will determine $\kappa=\nu+\alpha^{\prime}$. The operations $e_{i}^{\uparrow}$ can be applied by weakly decreasing indices $i$. For fixed $i$, the effect on rows $i, i+1$ of exhausting the operation $e_{i}^{\uparrow}$ is a transformation of the following form (with $\beta=\nu-\lambda$ ):

$$
\left(\begin{array}{ccccccccc}
0 & \cdots & 0 & \lambda_{i} & 0 & 0 & \cdots & 0 & \alpha_{i} \\
\beta_{0} & \cdots & \beta_{i-1} & \beta_{i} & \nu_{i+1} & 0 & \cdots & 0 & m
\end{array}\right) \stackrel{\left(e_{i}^{\uparrow}\right)^{*}}{\longrightarrow}\left(\begin{array}{ccccccccc}
\beta_{0} & \cdots & \beta_{i-1} & \nu_{i} & 0 & 0 & \cdots & 0 & m^{\prime} \\
0 & \ldots & 0 & 0 & \nu_{i+1} & 0 & \cdots & 0 & \alpha_{i+1}^{\prime}
\end{array}\right)
$$

where $\alpha_{i+1}^{\prime}=\min \left(m, \lambda_{i}-\nu_{i+1}\right)$, and $m^{\prime}=\alpha_{i}+m-\alpha_{i+1}^{\prime}$. The number $m$ is determined by the previous raising operations, except for the initial case $i=k-1$, where $m=$ $M_{k, l}$. Since the diagonal entry $\nu_{i}$ is unchanged by further applications of $e_{i-1}^{\uparrow}$, and $\nu_{i} \geq \lambda_{i} \geq \nu_{i+1}+\alpha_{i+1}^{\prime}$, there is no need to reapply $e_{i}^{\uparrow}$ later. Thus we have established a simple iterative procedure for determining the transformation of the final column of the matrix, which will then determine $\kappa$.

We rewrite this procedure into an algorithm for directly computing $\kappa$ from $\lambda$, $\mu, \nu$, and $M_{k, l}$, by taking into account $\alpha=\mu-\lambda$ and $\kappa=\nu+\alpha^{\prime}$; in this procure the roles of $\mu$ and of $\nu$ will be symmetrical. Adding $\nu_{i+1}$ to the equation for $\alpha_{i+1}^{\prime}$ 
we obtain $\kappa_{i+1}=\min \left(m+\nu_{i+1}, \lambda_{i}\right)$. The amount $m^{\prime}-\alpha_{i}$ added to $\alpha_{i}$ is equal to $m-\alpha_{i+1}^{\prime}=m+\nu_{i+1}-\kappa_{i+1}$; we shall use this "carry" $c$ in our algorithm rather than $m$. To that end we replace $m$ in our description by the sum of the original entry $\alpha_{i+1}=\mu_{i+1}-\lambda_{i+1}$ and the value $c$ left by the previous step; initially we take that expression with $c=M_{k, l}$. Then we get $\kappa_{i+1}=\min \left(d, \lambda_{i}\right)$ where $d=\mu_{i+1}-\lambda_{i+1}+c+\nu_{i+1}$, and $d-\kappa_{i+1}$ will replace $c$. The index $i+1$ now occurs much more often than $i$, and we shall therefore shift the index one up, so that $i+1$ becomes $i$. Finally, the last part of $\kappa$ that can be nonzero is $\kappa_{\min (k, l)}$, so we can start at $i=\min (k, l)$ instead of at $i=k$ and gain in efficiency and symmetry. After these transformations we get the following algorithm:

$$
\begin{aligned}
& c:=M_{k, l} ; \\
& \text { for } i \text { from } \min (k, l) \text { down to } 1 \\
& \quad \text { do } d:=\mu_{i}+\nu_{i}-\lambda_{i}+c ; \kappa_{i}:=\min \left(d, \lambda_{i-1}\right) ; c:=d-\kappa_{i} \text { od } ; \\
& \kappa_{0}:=\mu_{0}-\lambda_{0}+c+\nu_{0} .
\end{aligned}
$$

If we execute the algorithm for the values of the example above $\lambda=(8,4,2), \quad \mu=$ $(8,7,2), \quad \nu=(8,5,3,1), k=3, \quad l=6$, and $M_{3,6}=2$ then we find for $i=2: d:=3$, $\kappa_{3}=2, c:=1$; for $i=1: d:=4, \kappa_{2}=4, c:=0$; for $i=0: d:=8, \kappa_{1}=8, c:=0$, and finally $\kappa_{0}=8$, so that indeed one finds $\kappa=(8,8,4,2)$.

Note that the algorithm ensures that $\kappa_{i+1} \leq \lambda_{i}$ for all $i$, which means that $\lambda-\kappa$ will always hold; this is a stronger condition than follows just from the known relations $\lambda \leftarrow \mu-\kappa$ and $\lambda-\nu \leftarrow \kappa$. In fact the correspondence between the four partitions and the entry $M_{k, l}$ can be characterised by the following properties: (1) there exist numbers $c_{i} \in \mathbf{N}$ for $0 \leq i \leq \min (k, l)$ such that putting $c_{-1}=0$ one has $c_{i}+\mu_{i}+\nu_{i}=$ $\lambda_{i}+\kappa_{i}+c_{i-1}$ for all those $i ;(2) \kappa_{i+1} \leq \lambda_{i}$ for all $i$, and $c_{i}>0$ implies $\kappa_{i+1}=\lambda_{i}$; (3) $c_{\min (k, l)}=M_{k, l}$. For $i>0, c_{i}$ will be the value of $c$ in the algorithm at the start of the loop indexed by $i$, while $c_{0}$ is the final value of $c$. One may eliminate the dependence of this statement on $(k, l)$ by having $i$ range over $\mathbf{N}$ in property (1) and replacing property (3) by $\lim _{i \rightarrow \infty} c_{i}=M_{k, l}$ (in this case the sequence $c_{i}$ becomes stationary for $i \geq \min (k, l))$. This characterisation is useful for deducing the following algorithm inverse to the one above, which will compute $\lambda$ and $M_{k, l}$ from $\mu, \nu$, and $\kappa$.

$$
\begin{aligned}
& c:=0 ; \\
& \text { for } i \text { from } 0 \text { to } \min (k, l) \\
& \quad \operatorname{do} d:=\mu_{i}+\nu_{i}-\kappa_{i}-c ; \lambda_{i}:=\max \left(d, \kappa_{i+1}\right) ; c:=\lambda_{i}-d \text { od } \\
& M_{k, l}:=c .
\end{aligned}
$$

Executing this with as above $\kappa=(13,9,9,5), \mu=(11,9,8), \nu=(10,9,8,2), k=3$ and $l=4$ gives for $i=0: d:=8, \lambda_{0}=9, c:=1$; for $i=1: d:=8, \lambda_{1}=9, c:=1$; for $i=2$ : $d:=6, \lambda_{2}=6, c:=0$; for $i=3: d:=-3, \lambda_{3}=0, c:=3$, and finally $M_{3,4}=3$, which together with $\lambda=(9,9,6)$ is the result found before.

It should come as no surprise that the shape datum we have just described is in fact the shape datum for the Burge correspondence, which was given in [vLee4, $§ 3.2]$; after all we know from proposition 3.3.2 that the implicit shape computed for $M \in \mathcal{M}$ 


\subsection{Growth diagrams for integral matrices}

by the growth diagram for our shape datum is the shape of the tableaux associated to $M$ under the Burge correspondence. Although none of the descriptions given in that paper coincide exactly with the one given in (40), it is not hard to see their equivalence.

Those descriptions compute $[\kappa]$ by making a bottom-left to top-right pass over the squares that could be added to $[\lambda]$ (namely the topmost squares of the columns of the complement of that diagram), using a "stock of squares" whose size may grow and shrink. Initially the stock contains $M_{k, l}$ squares, and at each square $s$ visited, a square is added to the stock if $s \in[\mu / \lambda]$ and if $s \in[\nu / \lambda]$ (if both conditions hold, two squares are added), and then a square from the stock is contributed to $[\kappa]$ unless the stock is empty (so if $s$ lies either in $[\mu / \lambda]$ or $[\nu / \lambda]$ it will certainly be in $[\kappa]$, and if it lies in both there will in addition be a net increase of one square in the stock; if it lies in neither and the stock was not empty then $s$ will still be in $[\kappa]$ while the size of the stock is decremented). Algorithm (40) can be seen to compute this same diagram $[\kappa]$ a whole row at the time. The value $d=\mu_{i}+\nu_{i}-\lambda_{i}+c=\lambda_{i}+c+(\mu-\lambda)_{i}+(\nu-\lambda)_{i}$ represents the maximal length row $i$ of $[\kappa]$ can have, obtained by adding to $\lambda_{i}$ the size of the stock when arriving at this row and the sizes of rows $i$ of $[\mu / \lambda]$ and of $[\nu / \lambda]$. Either that length does not exceed the limit $\lambda_{i-1}$, in which case the one sets $\kappa_{i}=d$ and the stock becomes empty, or $d$ does exceed $\lambda_{i-1}$, in which case $\kappa_{i}$ is set to that limit and the size of the stack becomes $d-\lambda_{i-1}=d-\kappa_{i}$.

It is interesting to see how this description of the shape datum for the Burge correspondence arises naturally from the rules governing the normalisation process of integral matrices. Summarising, we have:

4.2.2. Theorem. If for any $M \in \mathcal{M}$ one associates to each grid point $(i, j) \in \mathbf{N}^{2}$ the shape $\pi\left(M_{[i] \times[j]}\right)$, then one obtains a growth diagram for the shape datum of the Burge correspondence.

Having determined the shape datum involved, we can now determine the family of shapes $\pi\left(M_{[i] \times[j]}\right)$ directly from the matrix $M$, without actually performing any crystal operations: it suffices to use the fact that $\pi\left(M_{[i] \times[j]}\right)=(0)$ when $i=0$ or $j=0$, and to construct the growth diagram for the shape datum. Diagram 1 illustrates this for the integral matrix $M$ of our running example.

From this growth diagram one not only can read off $\pi(M)$ in the bottom right corner, but also the matrices $P, Q$ associated to $M$ under the bijection of 3.1.3(2). To see that, observe that the operation $M \mapsto P$ of exhausting upward transfers commutes with the operation $M \mapsto M_{\mathbf{N} \times[j]}$ of discarding all columns beyond a certain point; this is just the fact observed above that $n_{i}^{\uparrow}(U \mid V)=0$ implies $n_{i}^{\uparrow}(U)=0$. Therefore the matrix $P_{\mathbf{N} \times[j]}$ can be obtained from $M_{\mathbf{N} \times[j]}$ by exhausting upward transfers, and one has $\operatorname{row}\left(P_{\mathbf{N} \times[j]}\right)=\pi\left(P_{\mathbf{N} \times[j]}\right)=\pi\left(M_{\mathbf{N} \times[j]}\right)$. Putting $\lambda^{(j)}=\operatorname{row}\left(P_{\mathbf{N} \times[j]}\right)$ for all $j$ defines the semistandard Young tableau $S=\left(\lambda^{(j)}\right)_{j \in \mathbf{N}}$ of which $P$ is the integral encoding, and we see that $S$ coincides with the sequence $\left(\pi\left(M_{\mathbf{N} \times[j]}\right)\right)_{j \in \mathbf{N}}$ that can be read off along the lower border of the growth diagram. Similarly $Q^{t}$ is the integral encoding of the semistandard Young tableau $\bar{L}=\left(\pi\left(M_{[i] \times \mathbf{N}}\right)\right)_{i \in \mathbf{N}}$ that can be read off along the right hand border. Indeed in the example of growth diagram 1, one finds $S$ of (31) and $\bar{L}$ of (33), whose integral encodings are the matrices $P$ and $Q^{t}$ of (29). 
4.2 Growth diagrams for integral matrices

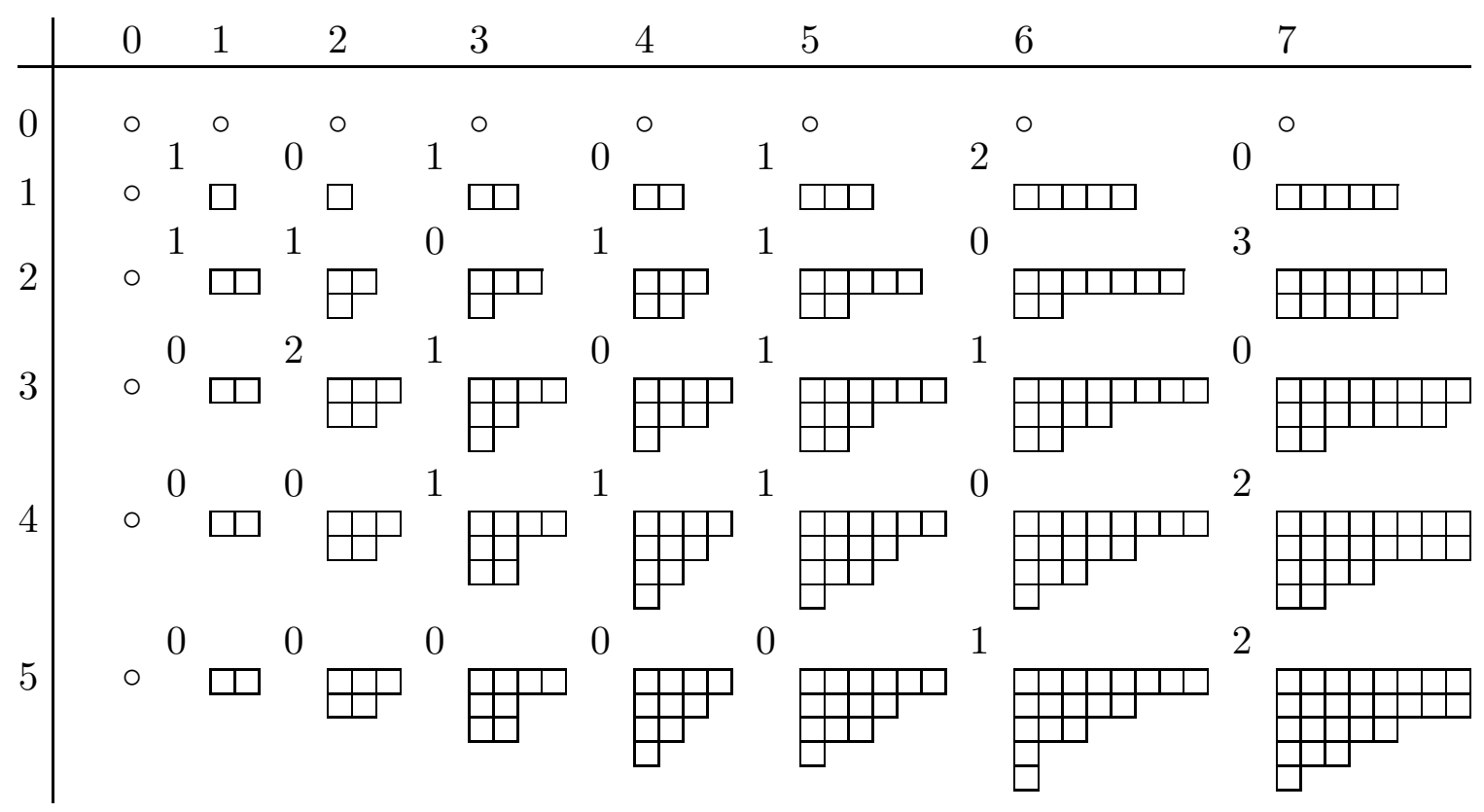

Diagram 1. Growth diagram with integral matrix and shapes $\left(\pi\left(M_{[i] \times[j]}\right)\right)_{i, j \in \mathbf{N}}$.

This argument confirms (and provides an alternative proof of) proposition 3.3.2, which can be interpreted as giving one particular way in which the growth diagram can be computed: column insertion from right to left of the letters of the weakly increasing word $w$ whose weight is given by the row $M_{i}$ gives the transition from the semistandard Young tableau $\left(\pi\left(M_{[i] \times[j]}\right)\right)_{j \in \mathbf{N}}$, formed by the shapes along row $i$ of the grid, to the tableau $\left(\pi\left(M_{[i+1] \times[j]}\right)\right)_{j \in \mathbf{N}}$ along the next row of the grid. Indeed one may check that the successive stages of the insertion process in (32) correspond to the shapes along the successive rows of the growth diagram above. (It may be a bit confusing that whereas the letters of $w$ are inserted from right to left, the growth diagram is extended from left to right; however, completing the square $(i, j)$ in the growth diagram does not correspond to the insertion of the letters $j$ of $w$, but rather to determining the effect on entries $\leq j$ of the insertion of $w$, relative to the effect of that insertion on entries $<j$.)

4.2.3. Summary. Given $M \in \mathcal{M}$, the shapes $\pi\left(M_{[i] \times[j]}\right)$ can be determined as follows for all $i, j \in \mathbf{N}$ (as there are only finitely many distinct matrices $M_{[i] \times[j]}$, the computation is effectively finite). Starting with $\pi\left(M_{[i] \times[j]}\right)=(0)$ whenever $i=0$ or $j=0$, one completes a growth diagram by repeatedly computing shapes $\kappa=\pi\left(M_{[i+1] \times[j+1]}\right)$ from previously computed shapes $\lambda=\pi\left(M_{[k] \times[j]}\right), \mu=\pi\left(M_{[k] \times[j+1]}\right)$, and $\nu=\pi\left(M_{[k+1] \times[j]}\right)$, and the entry $M_{k, l}$, using the algorithm (40). From this growth diagram one can read off the semistandard Young tableaux $S=\left(\pi\left(M_{\mathbf{N} \times[j]}\right)\right)_{j \in \mathbf{N}}$ and $\bar{L}=\left(\pi\left(M_{[i] \times \mathbf{N}}\right)\right)_{i \in \mathbf{N}}$ associated to $M$ under the Burge correspondence, as well as the matrices $P, Q$ associated to $M$ under the bijection of theorem 3.1.3(2): $P$ is the integral encoding of $S$ and $Q^{t}$ is the integral encoding of $\bar{L}$. One can reconstruct $M$ from $(P, Q)$ or from $(S, \bar{L})$ by reversing the computation, using algorithm (41) to compute $\lambda$ and $M_{k, l}$ given $\mu, \nu$, and $\kappa$. 


\subsection{Growth diagrams for binary matrices}

\subsection{Growth diagrams for binary matrices.}

We shall now consider the family of shapes $\pi\left(M_{[i] \times[j]}\right)$ for binary matrices. With respect to the integral case there is a complication, because one cannot in general reduce a submatrix $M_{[k] \times[l]}$ to its normal form using raising operations acting on the entire matrix. For instance after exhausting $e_{0}^{\leftarrow}, e_{1}^{\leftarrow}$, and $e_{0}^{\uparrow}$ for the binary matrix $M$ of (28), its submatrix $M_{[2] \times[3]}$ is reduced to $\left(\begin{array}{lll}1 & 1 & 0 \\ 1 & 0 & 1\end{array}\right)$ which is not a normal form; in isolation the bottom right entry could be moved up by $e_{0}^{\uparrow}$, but in the whole matrix it is blocked by the parts of rows 0 and 1 further to the right.

The difficulty comes from the reversal of left and right in the rule $n_{i}^{\uparrow}(U \mid V) \geq n_{i}^{\uparrow}(U)$ that we used for integral matrices: for binary matrices one instead has $n_{i}^{\uparrow}(U \mid V) \geq n_{i}^{\uparrow}(V)$ (for horizontal crystal operations there is no such difference with the integral case: the relation $n_{j}^{\leftarrow}\left(\frac{S}{T}\right) \geq n_{j}^{\leftarrow}(S)$ still holds for binary matrices). So we cannot exhaust upward moves in the submatrix $U$; however we have the rule $n_{i}^{\downarrow}(U \mid V) \geq n_{i}^{\downarrow}(U)$ that allows us to eventually exhaust any downward crystal operation $f_{i}^{\downarrow}$ on $U$ (after a finite number of applications of $f_{i}^{\downarrow}$ that move bits in $V$ ). One cannot exhaust all downward moves on a matrix, but if $k>0$ is such that $M=M_{[k] \times \mathbf{N}}$, then it will suffice to exhaust the operations $f_{i}^{\downarrow}$ for $i \in[k-1]$. If moreover leftward moves are also exhausted, then the matrix obtained from $M$ by a quarter turn clockwise, mapping the topmost $k$ rows to the leftmost $k$ columns, will satisfy the conditions of lemma 3.1.2; it follows that $M$ is obtained by the inverse rotation from $\operatorname{Diag}^{[2]}\left(\lambda^{t}\right)$, where $\lambda^{t}=\operatorname{col}(M)$, and in particular that $M$ is determined by its implicit shape $\lambda$ together with $k$. It is in fact an upside-down version of $\operatorname{Diag}^{[2]}(\lambda)$, in other words the region occupied by its bits ' 1 ' is the French style display* of the diagram of $\lambda$, in which the parts of $\lambda$ correspond to the rows taken from bottom to top.

4.3.1. Definition. Let $\lambda \in \mathcal{P}$ and $k \in \mathbf{N}$ with $\lambda_{k}=0$. We denote by $\operatorname{NF}(\lambda, k)$ the binary matrix $([(k-1-i, j) \in[\lambda]])_{i, j \in \mathbf{N}}$, which is called the French normal form (relative to $k$ ) parametrised by $\lambda$.

Note that $\operatorname{col}(\mathrm{NF}(\lambda, k))=\lambda^{t}$, while $\operatorname{row}(\mathrm{NF}(\lambda, k))=\left(\lambda_{k-1}, \ldots, \lambda_{0}\right)$; we shall write this composition obtained by reversing the $k$ initial parts of $\lambda$ as $\operatorname{rev}_{k}(\lambda)$. Now by exhausting the crystal operations in $\left\{f_{i}^{\downarrow} \mid i \in[k-1]\right\} \cup\left\{e_{j}^{\leftarrow} \mid j \in[l-1]\right\}$, the submatrix $M_{[k] \times[l]}$ of any binary matrix can be reduced to the form $\operatorname{NF}(\lambda, k)$, and this will be for $\lambda=\pi\left(M_{[k] \times[l]}\right)$. We call this process French normalisation of the submatrix $M_{[k] \times[l]}$. For instance for the binary matrix $M$ of $(28)$ and $(k, l)=(6,4)$, this exhaustion of crystal operations gives:

* One might have imagined that the occurrence of Young diagrams inside matrices, as in lemma 3.1.2, would give a coup de grâce to the French style of displaying diagrams. Mais non. 


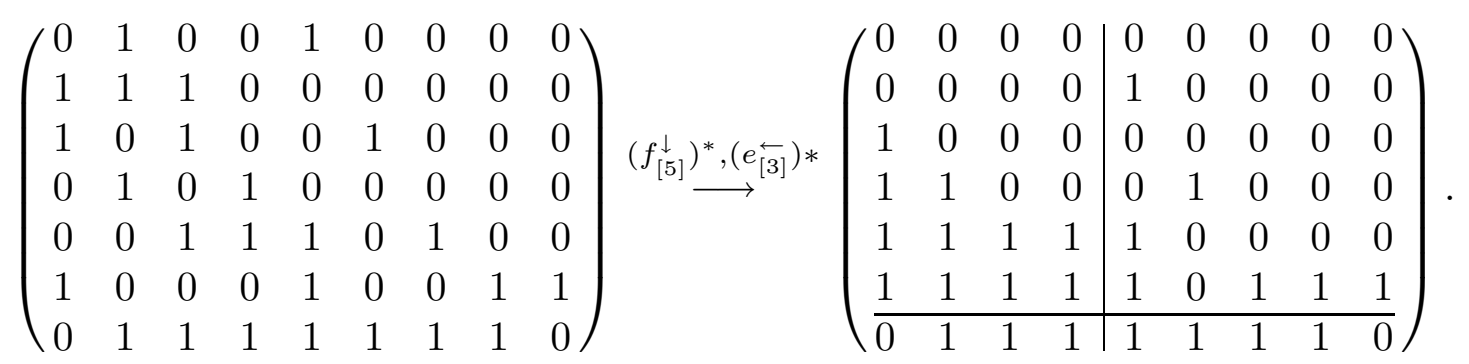

We see the submatrix $M_{[6] \times[4]}$ has been transformed into $\mathrm{NF}((4,4,2,1), 6)$, whence we may conclude that $\pi\left(M_{[6] \times[4]}\right)=(4,4,2,1)$. As in the integral case, we shall now compare the results of the French normalisation of submatrices $M_{[i] \times[j]}$ for $(i, j) \in$ $\{k, k+1\} \times\{l, l+1\}$. Let $A$ be obtained from $M_{[k+1] \times[l+1]}$ by exhausting the operations in $\left\{f_{i}^{\downarrow} \mid i \in[k-1]\right\} \cup\left\{e_{j}^{\leftarrow} \mid j \in[l-1]\right\}$, and as before put $\lambda=\pi\left(M_{[k] \times[l]}\right), \quad \mu=$ $\pi\left(M_{[k] \times[l+1]}\right), \quad \nu=\pi\left(M_{[k+1] \times[l]}\right)$, and $\kappa=\pi\left(M_{[k+1] \times[l+1]}\right)$. Then $A_{[k] \times[l]}=\mathrm{NF}(\lambda, k)$, and since French normalisation of $A_{[k] \times[l+1]}$ and of $A_{[k+1] \times[l]}$ only involves horizontal respectively vertical moves, and it transforms these submatrices into $\mathrm{NF}(\mu, k)$ respectively into $\mathrm{NF}(\nu, k+1)$, column $l$ of $A_{[k] \times[l+1]}$ is equal to the composition $\operatorname{rev}_{k}(\mu-\lambda)$, while row $k$ of $A_{[k+1] \times[l]}$ is equal to $\nu^{t}-\lambda^{t}$. In particular one always has $\lambda<\mu$ and $\lambda \leftarrow \nu$. In the example displayed above, where $\lambda=(4,4,2,1)$ and the $7 \times 5$ matrix $A$ can be read off from the matrix on the right, one finds $\mu=\lambda+(1,1,0,0,1,0)=(5,5,2,1,1)$ and $\nu=\left(\lambda^{t}+(0,1,1,1)\right)^{t}=(4,4,4,2)$.

As in the case of integral matrices we see that $A$, and therefore $\kappa=\pi(A)$, is determined by the shapes $\lambda, \mu, \nu$, and the bit $M_{k, l}$; moreover for any such data the construction provides an example $M=A$ of a matrix with the specified implicit shapes of submatrices and bit $M_{k, l}$, provided that one has $\lambda<\mu, \lambda \leftarrow \nu$, and $\mu_{k}=\nu_{l}^{t}=0$. By the same procedure as for integral matrices one can also reconstruct $\lambda$ and $M_{k, l}$ from $\mu, \nu$, and $\kappa$. Thus for any $\mu, \nu \in \mathcal{P}$ with $\mu_{k}=\nu_{l}^{t}=0$ one has a bijective correspondence between $\left(\lambda, M_{k, l}\right)$ on one hand and $\kappa$ on the other, where these values are restricted by the conditions $\lambda<\mu, \lambda-\nu, M_{k, l} \in[2], \nu \leftarrow \kappa$ and $\mu-\kappa$. Following the terminology of [vLee4, definition 6.1], these bijective correspondences for all $\mu, \nu \in \mathcal{P}$ together define an (asymmetric) shape datum for $(\mathcal{P}, \leftarrow, \leftarrow,[2])$. By assigning $\pi\left(M_{[i] \times[j]}\right)$ to each grid point $(i, j)$, one obtains a growth diagram for this asymmetric shape datum. Note that the shapes associated to grid points that are horizontal neighbours differ by a vertical strip, while those associated to vertical neighbours differ by a horizontal strip (we have no choice); in [vLee4] an opposite convention was used (its shape datum was for $(\mathcal{P}, \leftarrow, \leftarrow,[2])$, not for $(\mathcal{P}, \leftarrow, \leftarrow,[2]))$.

Before considering this shape datum more closely, let us compute an example. The example of $(42)$ for $(k, l)=(6,4)$ shows that during the French normalisation of $A_{[k+1] \times[l]}$ all bits ' 1 ' either remain in place or move one place down, while during the French normalisation of $A_{[k] \times[l+1]}$ some bits ' 1 ' may move several places to the left (the bit at $(1,4)$ moves all the way to $(1,0)$ ); however this example is too simple to illustrate well what can happen in general. Therefore consider instead $\lambda=(5,3,2)$, $\mu=(6,3,3,1,1), \nu=(6,3,2,2)$, and $M_{k, l}=1$ with $(k, l)=(5,6)$. For the corresponding matrix $A$ one has: 


$$
\begin{aligned}
& A=\left(\begin{array}{cccccc|c}
0 & 0 & 0 & 0 & 0 & 0 & 1 \\
0 & 0 & 0 & 0 & 0 & 0 & 1 \\
1 & 1 & 0 & 0 & 0 & 0 & 1 \\
1 & 1 & 1 & 0 & 0 & 0 & 0 \\
1 & 1 & 1 & 1 & 1 & 0 & 1 \\
\hline 1 & 1 & 0 & 0 & 0 & 1 & 1
\end{array}\right) \stackrel{\left(e_{[6]}^{\leftarrow}\right)^{*}}{\longrightarrow}\left(\begin{array}{ccccccc}
1 & 0 & 0 & 0 & 0 & 0 & 0 \\
1 & 0 & 0 & 0 & 0 & 0 & 0 \\
1 & 1 & 1 & 0 & 0 & 0 & 0 \\
1 & 1 & 1 & 0 & 0 & 0 & 0 \\
1 & 1 & 1 & 1 & 1 & 1 & 0 \\
\hline 1 & 1 & 0 & 1 & 0 & 0 & 1
\end{array}\right) \\
&\left(\begin{array}{cccccc|c}
0 & 0 & 0 & 0 & 0 & 0 & 1 \\
0 & 0 & 0 & 0 & 0 & 0 & 1 \\
1 & 1 & 0 & 0 & 0 & 0 & 0 \\
1 & 1 & 0 & 0 & 0 & 0 & 1 \\
1 & 1 & 1 & 0 & 0 & 0 & 1 \\
1 & 1 & 1 & 1 & 1 & 1 & 1
\end{array}\right) \stackrel{\left(f_{[5]}^{\downarrow}\right)^{*}}{\left.\stackrel{\left(f_{[6]}^{\leftarrow}\right.}{\longleftarrow}\right)^{*}} \underset{(43)}{\longrightarrow}\left(\begin{array}{ccccccc}
1 & 0 & 0 & 0 & 0 & 0 & 0 \\
1 & 0 & 0 & 0 & 0 & 0 & 0 \\
1 & 1 & 0 & 0 & 0 & 0 & 0 \\
1 & 1 & 1 & 0 & 0 & 0 & 0 \\
1 & 1 & 1 & 1 & 0 & 0 & 0 \\
1 & 1 & 1 & 1 & 1 & 1 & 1
\end{array}\right)=\mathrm{NF}(\kappa, 6),
\end{aligned}
$$

for $\kappa=(7,4,3,2,1,1)$. The parts of this computation that are not easy to predict are the following ones. Firstly there is, for the exhaustion of downward moves applied to $A$, the transformation of column $l$ of $A$ (which represents $\operatorname{rev}_{k}(\mu-\lambda)$ followed by $M_{k, l}$ ) into $\operatorname{rev}_{k+1}(\kappa-\nu)$; here some bits ' 1 ' move down a row (like in the example $M_{2,6}$; this corresponds to a square of $[\mu / \lambda]$ for which $[\kappa / \nu]$ contains a corresponding square in the same row), while some bits ' 1 ' remain in the same row (in the example bits $M_{0,6}$, $M_{1,6}, M_{4,6}$, and $M_{5,6}$; this corresponds to a square of $[\mu / \lambda]\left(\right.$ or $\left.M_{k, l}=1\right)$ for which $[\kappa / \nu]$ contains a corresponding square in the next row (respectively in row 0)). Secondly there is, for the exhaustion of leftward moves applied to $A$, the transformation of row $k$ of $A$ (which represents $\nu^{t}-\lambda^{t}$ followed by $M_{k, l}$ ) into $\kappa^{t}-\mu^{t}$; here the bits ' 1 ' may exchange with zero or more bits ' 0 ' initially situated directly to their left (in the example bit $M_{5,5}$ interchanges successively with two bits ' 0 ' to its left while $M_{5,0}, M_{5,1}$, and $M_{5,6}$ stay in place; such a bit corresponds to a square of $[\nu / \lambda]\left(\right.$ or $\left.M_{k, l}=1\right)$, and the corresponding square of $[\kappa / \mu]$ lies in a column weakly to the left of it (respectively in a column $\leq l)$ ).

Giving details of either of these transformations will give a description of how to compute $\kappa$. However, although these descriptions follow by a straightforward application of the definitions, they are rather technical, and both are not stated in the same terms (the former is primarily by rows of the shapes involved, the latter primarily by columns), which makes it hard to see that they give the same result for $\kappa$ (as we know they must). Therefore we shall instead describe this shape datum using the approach that was detailed in [vLee4, §6]; we have to adapt that description to the interchange of ' - ' and ' $\angle$ ' in the relations between shapes at horizontal and vertical neighbours in the grid.

Given $\mu, \nu \in \mathcal{P}$ for which there exists at least one $\lambda \in \mathcal{P}$ with $\mu \neg \lambda-\nu$, or equivalently at least one $\kappa \in \mathcal{P}$ with $\mu-\kappa \rightarrow \nu$, the possibilities for such $\lambda$ and $\kappa$ have a rather simple description: in each case the diagrams of these partitions are the disjoint union of a fixed set of "obligatory" squares and an arbitrary subset of a fixed set of "optional" squares. In case of $\lambda$, the Young diagram $[\mu] \cap[\nu]$ is the union of all obligatory and optional squares (and therefore the maximal choice for $[\lambda]$ ), and its subset $S$ of 
optional squares consists of those that are both at the end of a row of $[\mu]$ and at the end of a column of $[\nu]$, in equation: $S=\left\{(i, j) \in \mathbf{N}^{2} \mid(i, j)=\left(\nu_{j}^{t}-1, \mu_{i}-1\right)\right\}$. In the case of $\kappa$, the set of obligatory squares is the Young diagram $[\mu] \cup[\nu]$, and the set $T$ of optional squares consists of those that are both just beyond the end of a column of $[\mu]$ and just beyond the end of a row of $[\nu]$, in other words $T=\left\{(i, j) \in \mathbf{N}^{2} \mid(i, j)=\left(\mu_{j}^{t}, \nu_{i}\right)\right\}$. It was shown in [vLee4] that $\# T=\# S+1$, which is in agreement with the fact that there are twice as many possibilities for $\kappa$ as for $\lambda$; moreover, there is an injective map matching every $s \in S$ with the first $t \in T$ in a row strictly below $s$ (or equivalently in a column weakly to the left of $s$ ), which leaves the topmost square $t_{0}$ of $T$ unmatched. Now the correspondence between $\left(\lambda, M_{k, l}\right)$ and $\kappa$ is determined by the fact that for every optional square $s \in S$ absent from $[\lambda]$ the corresponding optional square $t \in T$ is present in $[\kappa]$, and vice versa, while one has $t_{0} \in[\kappa]$ if and only if $M_{k, l}=1$. We shall call this shape datum the "row-insertion shape datum for $(\mathcal{P}, \leftarrow, \leftarrow,[2])$ ".

4.3.2. Theorem. If for any $M \in \mathcal{M}^{[2]}$ one associates to each grid point $(k, l) \in \mathbf{N}^{2}$ the shape $\pi\left(M_{[k] \times[l]}\right)$, then one obtains a growth diagram for the row-insertion shape datum for $(\mathcal{P}, \longleftarrow, \leftarrow,[2])$.

We omit the proof; the assiduous reader may check that the rules governing French normalisation of submatrices do indeed lead to this shape datum. Whether one starts normalising $A_{[k+1] \times[l]}$ or $A_{[k] \times[l+1]}$, the description obtained will not directly give a correspondence between $S$ and $T$, but rather one between the sets of rows meeting $[\mu / \lambda]$ and $[\kappa / \nu]$, respectively between the sets of columns meeting $[\nu / \lambda]$ and $[\kappa / \mu]$. On one hand this means that a large number of steps may intervene between encountering the bit for some $s \in S$ and the one for the corresponding $t \in T$, and on the other hand the occurrence of such squares may be hidden by the fact that a square in $S$ shares a row or a column with a different square in $T$. As a consequence, cases need to be distinguished that play no role in the above description.

Now that the shape datum is known, it can be used to build up the growth diagram without actually performing (French) normalisation submatrices; again one starts by associating the empty partition (0) to every grid point $(i, j)$ with $i=0$ or $j=0$. The construction for the binary matrix $M$ of (28) is given in diagram 2, with the shapes associated to grid points given by their diagram à la française.

The shapes along the right border define the semistandard tableau $S$ of (31) whose binary encoding is the matrix $Q$ of (28), for essentially the same reasons as in the integral case: one has $\operatorname{col}\left(Q_{[i] \times \mathbf{N}}\right)=\pi\left(Q_{[i] \times \mathbf{N}}\right)^{t}=\pi\left(M_{[i] \times \mathbf{N}}\right)^{t}$ for all $i \in \mathbf{N}$. There is however no such direct relationship between matrix $P$ of (28) and the shapes along the lower border; this is not surprising since those shapes define a transpose semistandard tableau, whereas $P$ corresponds to the reverse transpose semistandard tableau $R$ of (35). Indeed, although like in the integral case one still has $\pi\left(P_{\mathbf{N} \times[j]}\right)=\pi\left(M_{\mathbf{N} \times[j]}\right)$ for all $j$, these shapes are not given by $\operatorname{row}\left(P_{\mathbf{N} \times[j]}\right)$, since upward moves need not be exhausted in $P_{\mathbf{N} \times[j]}$. To read off $R$ from a growth diagram, one should instead normalise the complementary submatrices $M_{\mathbf{N} \times(\mathbf{N}-[j])}$, as we shall do in the next subsection. On the other hand, our growth diagram can be interpreted as recording the insertion process of proposition 3.3.4, which gives as insertion tableau the Schützenberger dual $R^{*}$ of $R$ : 
4.3 Growth diagrams for binary matrices

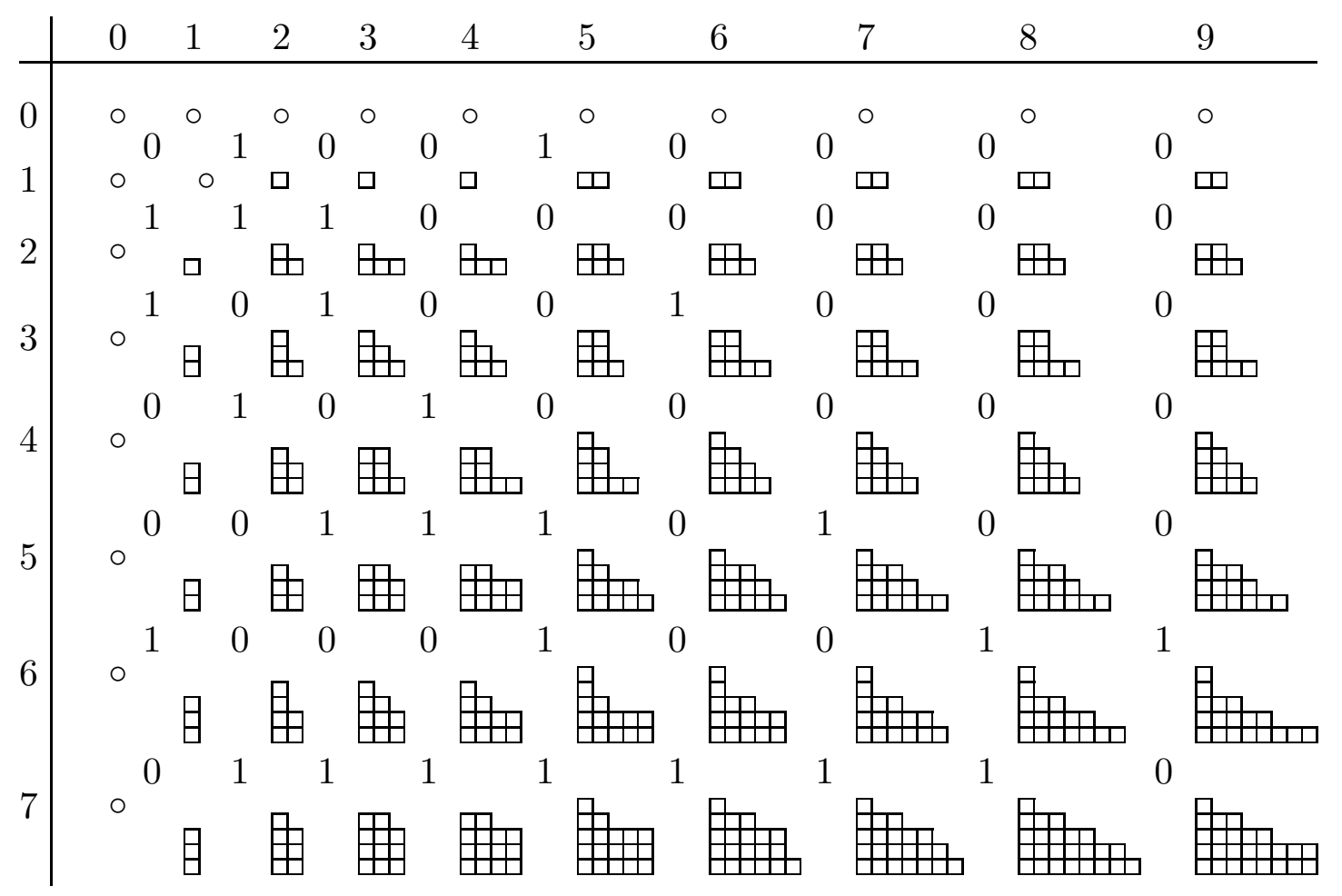

Diagram 2. Growth diagram with binary matrix and shapes $\left(\pi\left(M_{[i] \times[j]}\right)\right)_{i, j \in \mathbf{N}}$.

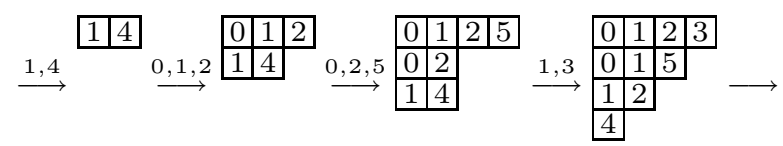

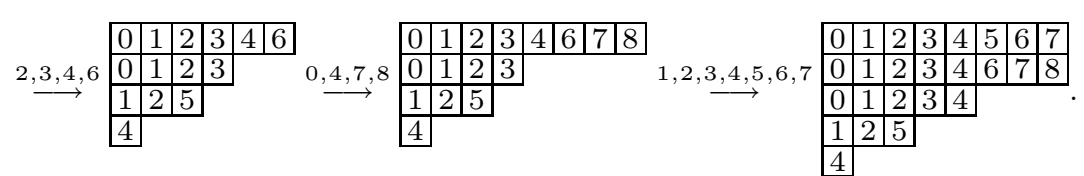

Indeed, since the shapes in our diagram are governed by the row-insertion shape datum for $(\mathcal{P}, \leftarrow, \leftarrow,[2])$, the passage from the transpose semistandard Young tableau defined by the shapes along row $i$ to those along row $i+1$ is given by the row insertion (according to the rules for transpose tableaux) of the letters of the strictly increasing word of weight $M_{i}$, from left to right. This proves:

4.3.3. Proposition. For $M \in \mathcal{M}^{[2]}$, the transpose semistandard tableau $R^{*}=$ $\left(\pi\left(M_{\mathbf{N} \times[j]}\right)\right)_{j \in \mathbf{N}}$ and the semistandard tableau $S=\left(\pi\left(M_{[i] \times \mathbf{N}}\right)\right)_{i \in \mathbf{N}}$ form the pair associated to $M$ in [Stan, theorem 7.14.2].

From $M$ one can obtain a matrix $\widetilde{P}$ whose rows give the weights of those of $R^{*}$, but in reverse order, by exhausting downward crystal operations, up to some limit. 
Exhausting $\left\{f_{i}^{\downarrow} \mid i \in[6]\right\}$ in our example gives

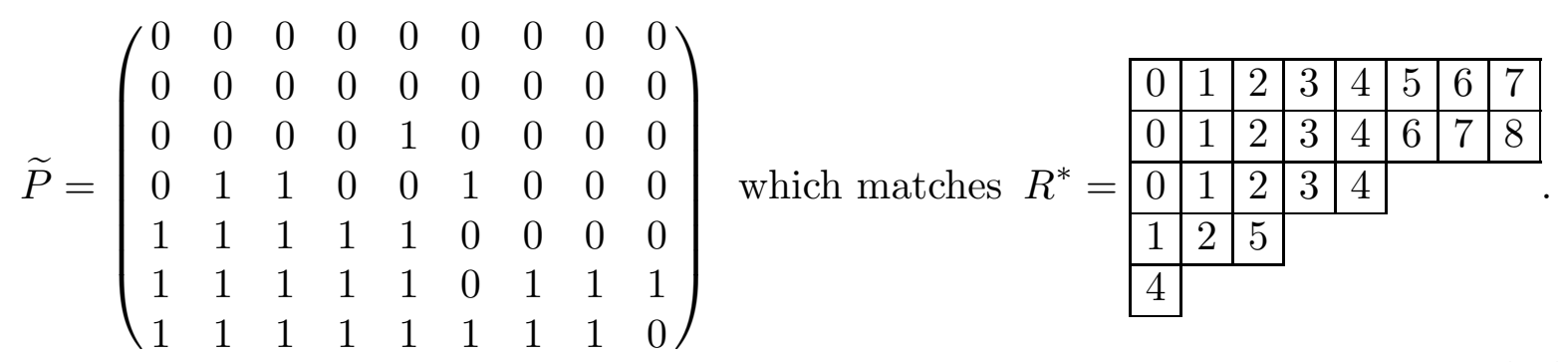

\subsection{Other types of growth diagrams.}

As was suggested above, one can form a growth diagram from which both tableaux $R$ and $S$ encoded by the matrices $P$ and $Q$ corresponding to $M \in \mathcal{M}^{[2]}$ under the bijection of theorem 3.1.3(1) can be directly read off, by computing the family of shapes $\left(\pi\left(M_{[i] \times(\mathbf{N}-[j])}\right)\right)_{i, j \in \mathbf{N}}$ rather than $\left(\pi\left(M_{[i] \times[j]}\right)\right)_{i, j \in \mathbf{N}}$. The shapes defining $R$, a weakly decreasing sequence in which adjacent shapes differ by vertical strips, can then be found along the bottom border, while the shapes defining $S$ are now found along the left border of the growth diagram. (In the direction from left to right one might prefer calling this a shrinkage diagram rather than a growth diagram; we shall call it a horizontally reversed growth diagram.) That $S$ will be found is obvious, since we are considering the implicit shapes of the same sequence of matrices; that fact that $R$ can be read off as indicated, follows from the fact that upward moves are exhausted in the submatrices $P_{\mathbf{N} \times(\mathbf{N}-[j])}$ for all $j$, whence one has $\operatorname{row}\left(P_{\mathbf{N} \times(\mathbf{N}-[j])}\right)=\pi\left(P_{\mathbf{N} \times(\mathbf{N}-[j])}\right)=\pi\left(M_{\mathbf{N} \times(\mathbf{N}-[j])}\right)$. We illustrate this in diagram 3 for the binary matrix $M$ of (28).

Calling this a horizontally reversed growth diagram means that for any pair of indices $k, l \in \mathbf{N}$, if we put $\lambda=\pi\left(M_{[k] \times(\mathbf{N}-[l+1])}\right), \quad \mu=\pi\left(M_{[k] \times(\mathbf{N}-[l])}\right), \quad \nu=$ $\pi\left(M_{[k+1] \times(\mathbf{N}-[l+1])}\right)$, and $\kappa=\pi\left(M_{[k+1] \times(\mathbf{N}-[l])}\right)$, there should be a relation between these shapes and the matrix entry $M_{k, l}$ that determines for fixed $\mu, \nu$ a bijection between the pair $\left(\lambda, M_{k, l}\right)$ and the shape $\kappa$. Indeed, if we determine the implicit shapes of submatrices by exhausting the pertinent raising operations (as in computing ordinary rather than French normal forms), then we have a situation in which everything is rotated a quarter turn clockwise with respect to that of the previous subsection. Taking our usual binary matrix $M$ of $(28)$ and $(k, l)=(5,2)$, we can read off the shapes $\lambda=\pi\left(M_{[5] \times(\mathbf{N}-[3])}\right)=(3,2,1), \quad \mu=\pi\left(M_{[5] \times(\mathbf{N}-[2])}\right)=(4,2,2,1)$, and $\nu=\pi\left(M_{[6] \times(\mathbf{N}-[3])}\right)=(5,2,2)$ from diagram 3 ; from this and $M_{5,2}=0$ we can compute $\kappa=(6,3,2,1)$ as follows (the two leftmost columns that are of no interest here are represented by dots): 


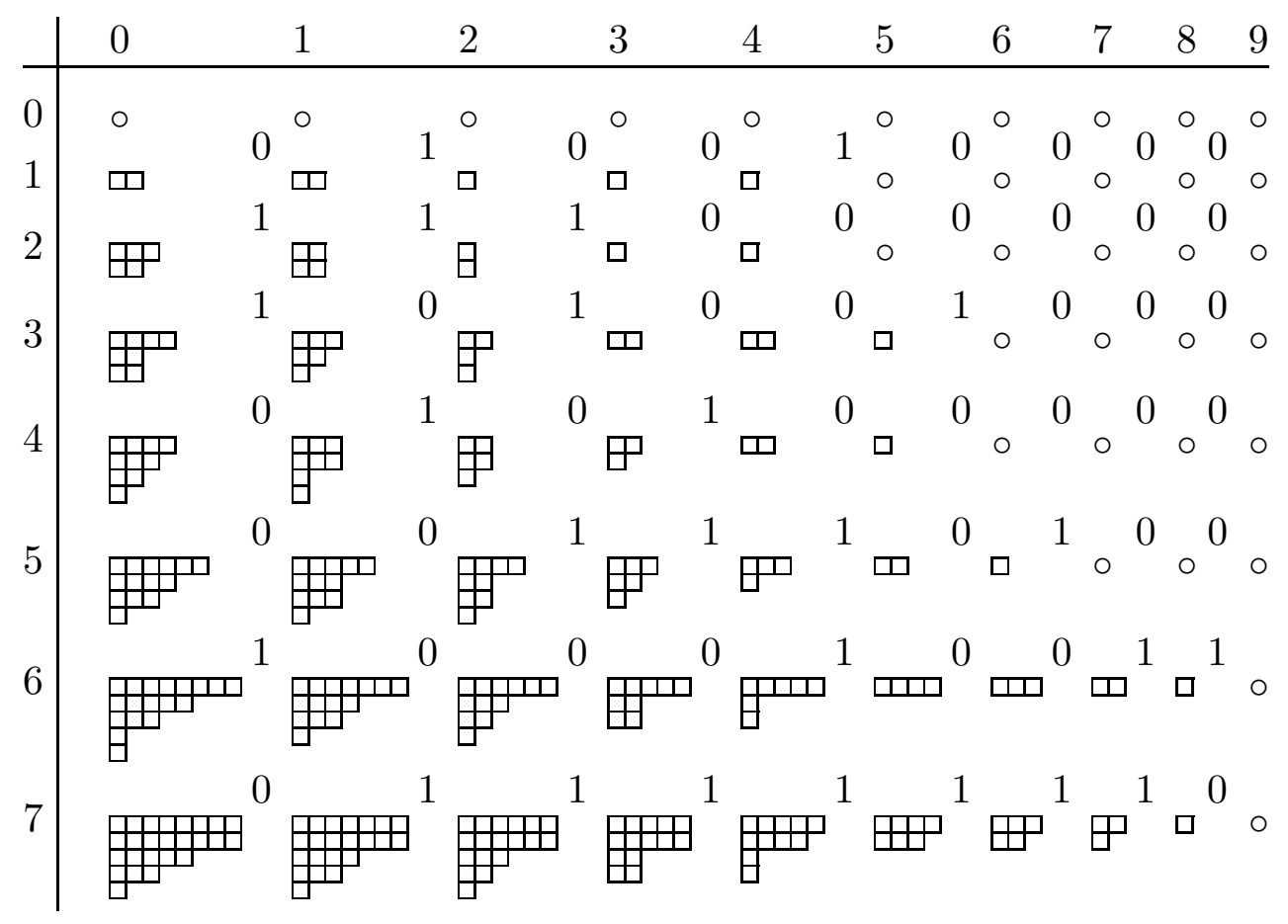

Diagram 3. Horizontally reversed growth diagram with binary matrix and shapes

$$
\left(\pi\left(M_{[i] \times(\mathbf{N}-[j])}\right)\right)_{i, j \in \mathbf{N}} .
$$

$$
\begin{aligned}
& \left(\begin{array}{lllllllll}
\cdot & \cdot & 1 & 1 & 1 & 1 & 0 & 0 & 0 \\
\cdot & \cdot & 1 & 1 & 0 & 0 & 0 & 0 & 0 \\
\cdot & \cdot & 1 & 1 & 0 & 0 & 0 & 0 & 0 \\
\cdot & \cdot & 1 & 0 & 0 & 0 & 0 & 0 & 0 \\
\cdot & \cdot & 0 & 0 & 0 & 0 & 0 & 0 & 0 \\
\hline . & \cdot & 0 & 0 & 1 & 0 & 1 & 1 & 0
\end{array}\right) \\
& \stackrel{\left(e_{\mathbf{N}-[2]}^{\leftarrow}\right)^{*}}{\longleftarrow}\left(\begin{array}{ccc|cccccc}
\cdot & \cdot & 1 & 1 & 1 & 1 & 0 & 0 & 0 \\
\cdot & \cdot & 0 & 1 & 1 & 0 & 0 & 0 & 0 \\
\cdot & \cdot & 1 & 1 & 0 & 0 & 0 & 0 & 0 \\
\cdot & \cdot & 1 & 0 & 0 & 0 & 0 & 0 & 0 \\
\cdot & \cdot & 0 & 0 & 0 & 0 & 0 & 0 & 0 \\
\hline \cdot & \cdot & 0 & 0 & 1 & 0 & 1 & 1 & 0
\end{array}\right) \\
& \left(e_{[5]}^{\uparrow}\right)^{*} \\
& \left(e_{[5]}^{\uparrow}\right)^{*} \downarrow \\
& \left(\begin{array}{lllllllll}
\cdot & \cdot & 1 & 1 & 1 & 1 & 1 & 1 & 0 \\
\cdot & \cdot & 1 & 1 & 1 & 0 & 0 & 0 & 0 \\
\cdot & \cdot & 1 & 1 & 0 & 0 & 0 & 0 & 0 \\
\cdot & \cdot & 1 & 0 & 0 & 0 & 0 & 0 & 0 \\
\cdot & \cdot & 0 & 0 & 0 & 0 & 0 & 0 & 0 \\
. & \cdot & 0 & 0 & 0 & 0 & 0 & 0 & 0
\end{array}\right) \\
& \stackrel{\left(e_{\mathbf{N}-[2]}^{\leftarrow}\right)^{*}}{\longleftarrow}\left(\begin{array}{ccc|cccccc}
\cdot & \cdot & 1 & 1 & 1 & 1 & 1 & 1 & 0 \\
\cdot & \cdot & 1 & 1 & 1 & 0 & 0 & 0 & 0 \\
\cdot & \cdot & 0 & 1 & 1 & 0 & 0 & 0 & 0 \\
\cdot & \cdot & 1 & 0 & 0 & 0 & 0 & 0 & 0 \\
\cdot & \cdot & 0 & 0 & 0 & 0 & 0 & 0 & 0 \\
\cdot & \cdot & 0 & 0 & 0 & 0 & 0 & 0 & 0
\end{array}\right) .
\end{aligned}
$$

The rotation of the matrices induces the transposition of their implicit shapes and those of their submatrices; therefore the relation here between $\lambda^{t}, \nu^{t}, \mu^{t}$, and $\kappa^{t}$ (in that order) will be the same as was found between $\lambda, \mu, \nu$, and $\kappa$ in the situation of the previous subsection. It follows that one still has $\lambda<\mu \leftarrow \kappa$ and $\lambda \leftarrow \nu<\kappa$ (which is also obvious from the computation considered), but the shape datum that determines $\kappa$ will be the column-insertion shape datum for $(\mathcal{P}, \leftarrow, \leftarrow,[2])$; its definition differs from the row-insertion shape datum by the fact that another injective map $S \rightarrow T$ is used, one 
that maps each $s \in S$ to the first $t \in T$ in a column strictly to its right (and in a row weakly above it).

4.4.1. Proposition. The bijection of theorem 3.1.3(1) between $M \in \mathcal{M}^{[2]}$ and the pair $(P, Q)$, or the pair of tableaux $(R, S)$ encoded by them as in proposition 3.3.3, can be computed using a horizontally reversed growth diagram for the column-insertion shape datum for $(\mathcal{P}, \leftarrow, \leftarrow,[2])$, reading off $R=\left(\pi\left(M_{\mathbf{N} \times(\mathbf{N}-[j]}\right)\right)_{j \in \mathbf{N}}$ along the lower border and $S=\left(\pi\left(M_{[i] \times \mathbf{N}}\right)_{i \in \mathbf{N}}\right.$ along the left border.

We may interpret proposition 3.3.3 as a way to determine such horizontally reversed growth diagrams: the transition from the semistandard Young tableau formed by the shapes along column $j+1$ of the grid and the one formed by those along column $j$ is given by column insertion in strictly increasing order of the letters of a word $w$ whose weight is given by the column $M_{j}^{t}$. This may be verified by comparing the intermediate tableaux in (34) with the vertical sequences of shapes in the display above. Computing the shape $\kappa$ at the bottom left corner of a square $(i, j)$ amounts to finding the shape occupied by letters $\leq i$ after column-insertion of $w$, given the shape $\mu$ occupied by letters $<i$ after that insertion, the horizontal strip $\nu / \lambda$ filled with letters $i$ before the insertion, and the number $M_{i, j} \in\{0,1\}$ of letters $i$ in $w$.

We have seen how $\pi(M)$ can be computed using either an ordinary or a horizontally reversed growth diagram, which were determined by studying the exhaustion of appropriate crystal operations on submatrices from the top left respectively from the top right, and the two situations are the same up to a rotation of everything by a quarter turn. It should by now be clear that two more variations to this construction can be obtained by further rotations. Thus we obtain computations using a vertically and a doubly reversed growth diagram, which are respectively governed by the columninsertion an by the row-insertion shape datum for $(\mathcal{P}, \leftarrow, \leftarrow,[2])$. The relations this gives between the results of applying the corresponding variations of the dual RSK algorithm to $M$ are both straightforward and in principle known (see for instance [Fult]), so we shall not give more details here. However, for integral matrices similarly defined variant growth diagrams are not all related by simple symmetries, as we shall now see.

For integral matrices $M \in \mathcal{M}$ we have seen that any upper left submatrix $M_{[i] \times[j]}$ can be reduced inside $M$ to its normal form by exhausting the applicable upward and leftward crystal operations. If one views $M$ as a finite rectangular matrix, then one can similarly bring any submatrix containing one of the three remaining corners into a standard form by exhausting an appropriate set of crystal operations, but the result is not the same in all cases. In the case of a lower right submatrix the situation is rotated a half turn with respect to that of an upper left submatrix, so exhaustion of all downward and rightward crystal operations between pairs of rows respectively columns of the submatrix will end with a rotated version of $\operatorname{Diag}(\lambda)$ : all units are on the diagonal passing through the bottom right corner, their numbers weakly increasing towards that corner. This rotated version has the same implicit shape $\lambda$ as $\operatorname{Diag}(\lambda)$, as can be verified by any of the many ways we have seen to compute implicit shapes. Therefore the family of shapes $\left(M_{(\mathbf{N}-[i]) \times(\mathbf{N}-[j])}\right)_{i, j \in \mathbf{N}}$ defines a doubly reversed growth diagram in, which for all $k, l \in \mathbf{N}$ the four shapes $\lambda=\pi\left(M_{(\mathbf{N}-[k+1]) \times(\mathbf{N}-[l+1])}\right), \quad \mu=$ 


\subsection{Other types of growth diagrams}

$\pi\left(M_{(\mathbf{N}-[k+1]) \times(\mathbf{N}-[l])}\right), \quad \nu=\pi\left(M_{(\mathbf{N}-[k]) \times(\mathbf{N}-[l+1])}\right)$, and $\kappa=\pi\left(M_{(\mathbf{N}-[k]) \times(\mathbf{N}-[l])}\right)$ at the corners of a grid square and the entry $M_{k, l}$ of that square are related by the shape datum of the Burge correspondence, that can be computed by algorithms (40) and (41).

For submatrices containing the top right or bottom left corner of $M$ however, the situation is not symmetric to those of the top left and bottom right corners; on the other hand these two remaining cases are related by a rotation by a half turn, so we shall focus (arbitrarily) on the bottom left submatrices. A normalised form for the submatrix $M_{(\mathbf{N}-[k]) \times[l]}$ that can be obtained by crystal operations acting on the entire matrix, is the one where upward and rightward crystal operations between pairs of rows respectively columns of the submatrix are exhausted, in other words the operations in $\left\{e_{i}^{\uparrow} \mid i \in \mathbf{N}-[k]\right\} \cup\left\{f_{j} \mid j \in[l-1]\right\}$. The result is not however that all units of the submatrix end up on one same diagonal or anti-diagonal, as we illustrate for our usual integral matrix $M$ and its submatrix $(k, l)=(1,6)$ :

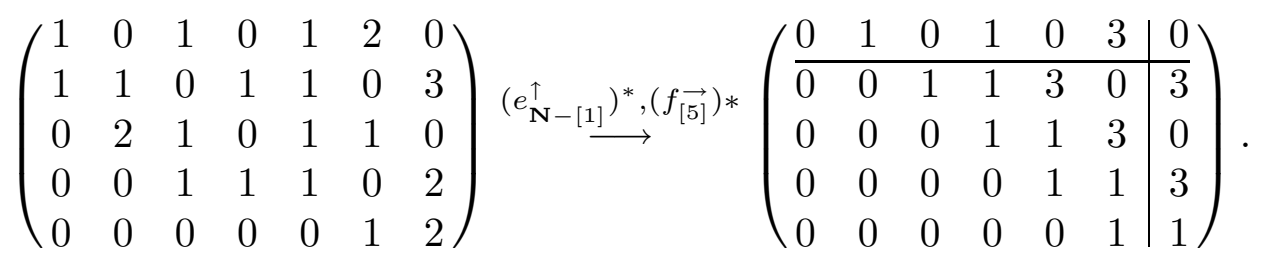

One sees that entries $M_{i, j}$ with $(i, j) \in(\mathbf{N}-[k]) \times[l]$ have become constant along diagonals, and the implicit shape of $M_{(\mathbf{N}-[k]) \times[l]}$ can be read off from the row sums of the submatrix after the transformation: in (47) on has $\pi\left(M_{(\mathbf{N}-[1]) \times[6]}\right)=(5,5,2,1)$. This must be so, for if one would isolate $M_{(\mathbf{N}-[k]) \times[l]}$ and exhaust leftward crystal operations on it, then the result would on one hand be a shifted version of Diag $(\lambda)$ for $\lambda=\pi\left(M_{(\mathbf{N}-[k]) \times[l]}\right)$, and on the other hand it would have unchanged row sums. Whereas the $\lambda_{i}$ units forming such a row sum are all grouped together in one entry of $\operatorname{Diag}(\lambda)$, they are distributed in the corresponding row of $M_{(\mathbf{N}-[k]) \times[l]}$ according to the partitioning of the squares of row $i$ of $[\lambda]$ by the lengths of their columns: the unit corresponding to a square lies on the diagonal near the top right of $(\mathbf{N}-[k]) \times[l]$ that has the same length as the column of the square. This is easily checked, since the entry $M_{k+i, l-1}$ lies at the end of the row of $M_{(\mathbf{N}-[k]) \times[l]}$ with sum $\lambda_{i}$ and also of a diagonal of length $i+1$, and its value is $M_{k+i, l-1}=\lambda_{i}-\lambda_{i+1}$, which is the number of columns of length $i+1$ of the diagram $[\lambda]$. Thus the submatrix shows how that diagram can be sliced vertically into parts for each column length; for instance in the example the diagram $[(5,5,2,1)]$ appears as cut up into two single columns, of length 4 and 3 , and a group of the remaining 3 columns of length 2 , while there are 0 columns of length 1 . We now define more formally the "sliced form" matrix that thus corresponds to $\lambda$, for given $k, l$.

4.4.2. Definition. Let $\lambda \in \mathcal{P}$ and $k, l \in \mathbf{N}$ with $\lambda_{l}=0$. We denote by $\operatorname{SF}(\lambda, k, l)$ the integral matrix $M$ defined by $M_{i, j}=[i \geq k][j<l]\left(\lambda_{m-1}-\lambda_{m}\right)$ for $i, j \in \mathbf{N}$, where $m=i-k-j+l$.

4.4.3. Proposition. Let $k, l \in \mathbf{N}$ with $l>0$ be fixed; then $M \in \mathcal{M}$ satisfies $M=$ $M_{(\mathbf{N}-[k]) \times[l]}, \quad n_{i}^{\uparrow}(M)=0$ for $i \geq k$, and $n_{j}(M)=0$ for $j \in[l-1]$, if and only if there exists $\lambda \in \mathcal{P}$ with $\lambda_{l}=0$ such that $M=\mathrm{SF}(\lambda, k, l)$. 
Proof. It is clear that any $M=\mathrm{SF}(\lambda, k, l)$ with $\lambda_{l}=0$ satisfies the given conditions: for that case all the contributions to the sums in (18) and (21) are $\leq 0$. Conversely suppose that $M$ satisfies those conditions. If its entries were not constant along all diagonals within $(\mathbf{N}-[k]) \times[l]$, then on the lowest diagonal with non-constant entries one could find a pair $M_{i, j} \neq M_{i+1, j+1}$ with $k \leq i$ and $j+1<l$; if $M_{i, j}<M_{i+1, j+1}$ one would have $n_{i}^{\uparrow}(M)>0$ while if $M_{i, j}>M_{i+1, j+1}$ one would have $n_{j}(M)>0$, either of which contradicts the assumption. Therefore the entries of $M$ are constant along such diagonals; moreover $n_{i}^{\uparrow}(M)=0$ implies $M_{i+1,0}=0$ for all $i \geq k$, so the entries are zero on all diagonals below the one containing $M_{k, 0}$. Then putting $\lambda_{i}=\sum_{j \in[l]} M_{k+i, j}$ one checks that $\lambda \in \mathcal{P}, \quad \lambda_{l}=0$, and $M=\operatorname{SF}(\lambda, k, l)$.

Now consider an integral matrix $A=A_{(\mathbf{N}-[k]) \times[l+1]}$ that satisfies $n_{i}^{\uparrow}(A)=n_{j}(A)=$ 0 for all $i \geq k+1$ and $j<l-1$, so that in particular its submatrix $A_{(\mathbf{N}-[k+1]) \times[l]}$ is of the form just described. Let us try to find the relation between the entry $A_{k, l}$ just above and to the right of that submatrix, and the shapes at the grid points forming the corners of its square: $\lambda=\pi\left(A_{(\mathbf{N}-[k+1]) \times[l]}\right), \quad \mu=\pi\left(A_{(\mathbf{N}-[k+1]) \times[l+1]}\right), \quad \nu=\pi\left(A_{(\mathbf{N}-[k]) \times[l]}\right)$, and $\kappa=\pi\left(A_{(\mathbf{N}-[k]) \times[l+1]}\right)$. The matrix after the transformation in (47) provides an example with $(k, l)=(0,6), M_{k, l}=0$, and $\lambda=(5,5,2,1)$; the computation below shows that in this case $\mu=(8,5,5,2), \nu=(8,5,3,1,1)$, and $\kappa=(8,8,5,3,1)$.

$$
\begin{aligned}
\left.\left(\begin{array}{cccccc|c}
0 & 1 & 0 & 2 & 2 & 3 & 0 \\
0 & 0 & 1 & 0 & 2 & 2 & 3 \\
0 & 0 & 0 & 1 & 0 & 2 & 2 \\
0 & 0 & 0 & 0 & 1 & 0 & 2 \\
0 & 0 & 0 & 0 & 0 & 1 & 0
\end{array}\right) \stackrel{\stackrel{\left(f_{[6]}\right)^{*}}{\longrightarrow}}{\left(e_{\mathbf{N}-[0]}^{\uparrow}\right.}\right)^{* \uparrow}\left(\begin{array}{cccccccc}
0 & 0 & 1 & 2 & 2 & 3 & 0 \\
0 & 0 & 0 & 1 & 2 & 2 & 3 \\
0 & 0 & 0 & 0 & 1 & 2 & 2 \\
0 & 0 & 0 & 0 & 0 & 1 & 2 \\
0 & 0 & 0 & 0 & 0 & 0 & 1
\end{array}\right)=\operatorname{SF}(\kappa, 0,7) \\
A= \\
\left(\begin{array}{cccccc|c}
0 & 1 & 0 & 1 & 0 & 3 & 0 \\
0 & 0 & 1 & 1 & 3 & 0 & 3 \\
0 & 0 & 0 & 1 & 1 & 3 & 0 \\
0 & 0 & 0 & 0 & 1 & 1 & 3 \\
0 & 0 & 0 & 0 & 0 & 1 & 1
\end{array}\right) \stackrel{\left(e_{\mathbf{N}-[0]}^{\uparrow}\right)^{*}}{\stackrel{\left(f_{[6]}\right)^{*}}{\longrightarrow}}\left(\begin{array}{ccccccc}
0 & 0 & 1 & 1 & 0 & 3 & 0 \\
0 & 0 & 0 & 2 & 3 & 0 & 3 \\
0 & 0 & 0 & 0 & 2 & 3 & 0 \\
0 & 0 & 0 & 0 & 0 & 2 & 3 \\
0 & 0 & 0 & 0 & 0 & 0 & 2
\end{array}\right)
\end{aligned}
$$

We shall now analyse in detail what happens during this procedure. The description will be heavy going at times, but it eventually leads to the shape datum for the RSK correspondence; as this forms the most direct link of our constructions to that correspondence, we insist on presenting it, with its inevitable technical details. The reader may choose to either bear with us, or take our word for it and skip to (53).

The parts of the transformations above that respectively reduce $A_{(\mathbf{N}-[k]) \times[l]}$ to $\mathrm{SF}(\nu, k, l)$ by upward transfers (the part to the left of the vertical line for the left upward arrow), and $A_{(\mathbf{N}-[k+1]) \times[l+1]}$ to $\mathrm{SF}(\mu, k+1, l+1)$ by rightward transfers (below the horizontal line for the bottom arrow), are less transparent that the corresponding parts in (38), but we can describe the former as follows (the latter is quite similar). From $n_{j}(A)=0$ it follows that $A_{k, j} \leq A_{k+1, j+1}$ for $j \in[l-1]$. Therefore, if one starts by exhausting $n_{k}^{\uparrow}$ applied to $A$, the entry $A_{k, j}$ will block as many units of $A_{k+1, j+1}$; 


\subsection{Other types of growth diagrams}

any further units of $A_{k+1, j+1}$ however will not be permanently blocked, and they will eventually (when all transfers further to the right are completed) be transferred one row up to the entry $A_{k, j+1}$. As a result of this exhaustion, every entry $A_{k+1, j+1}$ will have become equal to the initial value of $A_{k, j}$, and $A_{k+1,0}$ will have become 0 . We illustrate this for the example above, showing just the first 6 columns of rows 0,1 :

$$
\left(\begin{array}{llllll}
0 & 1 & 0 & 1 & 0 & 3 \\
0 & 0 & 1 & 1 & 3 & 0
\end{array}\right) \stackrel{\left(e_{0}^{\uparrow}\right)^{*}}{\longrightarrow}\left(\begin{array}{llllll}
0 & 1 & 0 & 2 & 2 & 3 \\
0 & 0 & 1 & 0 & 1 & 0
\end{array}\right) .
$$

After this exhaustion, the situation in the first $l$ columns of rows $k+1, k+2$ is the same as the original situation in rows $k, k+1$, but shifted one place to the right so that the final entries disappear, and with entries 0 shifted in at the left. One can therefore continue by exhausting $n_{k+1}^{\uparrow}$, which will involve shifted copies of the same moves (except those that took place in the final column), and then similarly exhaust $n_{k+2}^{\uparrow}, \ldots, n_{k+l-1}^{\uparrow}$; after this, the entries in $A_{(\mathbf{N}-[k]) \times[l]}$ will have become constant along diagonals, so that submatrix is then equal to $\mathrm{SF}(\nu, k, l)$. Thus we see that row $k$ of the submatrix will already have obtained its final value after the initial exhaustion of $e_{k}^{\uparrow}$, and $\nu$ can already be read off from this row.

Now for any $j>0$, the initial value of $A_{k+1, l-j}$ represents the number $\lambda_{j-1}-\lambda_{j}$ of columns of length $j$ of $[\lambda]$, and the final value of the entry $A_{k, l-j}$ above it represents the number of columns of that length of $[\nu]$. Then the units that are transferred upwards from $A_{k+1, l-j}$ to $A_{k, l-j}$ represent columns whose length is $j$ in $[\lambda]$ and remains so in $[\nu]$, while the units that already were in $A_{k, l-j}$ initially (and remain there), and whose number equals the number of units that remain in $A_{k+1, l-(j-1)}$ if $j>1$, represent columns whose length grows from $j-1$ in $[\lambda]$ to $j$ in $[\nu]$. The latter columns are the ones that for which row $j-1$ of $[\nu / \lambda]$ contains a square. From this one deduces that $\nu / \lambda$ is a horizontal strip, and that the initial value of the reverse $\left(A_{k, l-1}, \ldots, A_{k, 0}\right)$ of the topmost row of $A_{(\mathbf{N}-[k]) \times[l]}$ gives the value of $\nu-\lambda$. In the example above one has $\nu-\lambda=(8,5,3,1,1,0)-(5,5,2,1,0,0)=(3,0,1,0,1,0)=\left(A_{0,5}, \ldots, A_{0,0}\right)$.

We might have reached the same conclusion with somewhat less effort by considering column sums, which are unaffected by upward transfers, but the details will be needed anyway to understand the full transformation of $A$ by upward moves, notably the changes to column $l$. We now know that this column initially contains, below the entry $A_{k, l}$, a vertical copy of the composition $\mu-\lambda$, and after the transformation it will contain a similar copy of $\kappa-\nu$ (but shifted one place less, starting at square $(k, l)$ ). When we consider the exhaustion of some $e_{k+i}^{\uparrow}$, we can focus exclusively on columns $l-1$ and $l$, since what we have seen above implies that at that time one has $A_{k+i, j} \leq A_{k+i+1, j+1}$ for all $j \in[l-1]$, so that the presence of columns to the left of column $l-1$ will not block any upward transfers in column $l$.

The first $2 \times 2$ submatrix to consider is $A_{\{k, k+1\} \times\{l-1, l\}}$ whose entries are initially (i.e., at the start of the exhaustion of $e_{k}^{\uparrow}$ ) the following: $A_{k, l-1}$ is the final entry $\nu_{0}-\lambda_{0}$ of the composition $\operatorname{rev}_{l}(\nu-\lambda)$ that is in the first $l$ columns of row $k ; A_{k, l}$ is arbitrary; $A_{k+1, l-1}$ is the top-rightmost entry $\lambda_{0}-\lambda_{1}$ of the submatrix $A_{(\mathbf{N}-[k+1]) \times[l]}=\mathrm{SF}(\lambda, k+$ $1, l) ; A_{k+1, l}$ is the top entry $\mu_{0}-\lambda_{0}$ of the vertical copy of $\mu-\lambda$ in column $l$. At the 
start of the exhaustion of $e_{k+i}^{\uparrow}$, the values in the $2 \times 2$ submatrix $A_{\{k+i, k+i+1\} \times\{l-1, l\}}$ are similarly: $A_{k+i, l-1}=\nu_{i}-\lambda_{i}$ is a copy of the initial value of $A_{k, l-1-i} ; A_{k+i, l}$ is some value $a$ left after the exhaustion of $e_{k+i-1}^{\uparrow}$ (which we shall determine presently); $A_{k+i+1, l-1}$ is the final entry $\lambda_{i}-\lambda_{i+1}$ of row $k+1+i$ of $\operatorname{SF}(\lambda, k+1, l)$; and finally $A_{k+i+1, l}=\mu_{i}-\lambda_{i}$. The upward moves in column $l$ are then given by

$$
\left(\begin{array}{cc}
\nu_{0}-\lambda_{0} & A_{k, l} \\
\lambda_{0}-\lambda_{1} & \mu_{0}-\lambda_{0}
\end{array}\right) \stackrel{\left(e_{k}^{\uparrow}\right)^{\max \left(\mu_{0}-\nu_{0}, 0\right)} \longrightarrow}{\longrightarrow}\left(\begin{array}{cc}
\nu_{0}-\lambda_{0} & A_{k, l}+\max \left(\mu_{0}-\nu_{0}, 0\right) \\
\lambda_{0}-\lambda_{1} & \min \left(\mu_{0}, \nu_{0}\right)-\lambda_{0}
\end{array}\right)
$$

for the transfer between rows $k$ and $k+1$ (which will be followed by a transfer in column $l-1$ changing it from $\left(\begin{array}{c}\nu_{0}-\lambda_{0} \\ \lambda_{0}-\lambda_{1}\end{array}\right)$ into $\left(\begin{array}{c}\nu_{0}-\nu_{1} \\ \nu_{1}-\lambda_{1}\end{array}\right)$, but that part was already known), and similarly in rows $k+i$ and $k+i+1$ :

$$
\left(\begin{array}{cc}
\nu_{i}-\lambda_{i} & a \\
\lambda_{i}-\lambda_{i+1} & \mu_{i}-\lambda_{i}
\end{array}\right) \stackrel{\left(e_{k+i}^{\uparrow}\right)^{\max \left(\mu_{i}-\nu_{i}, 0\right)}}{\longrightarrow}\left(\begin{array}{cc}
\nu_{i}-\lambda_{i} & a+\max \left(\mu_{i}-\nu_{i}, 0\right) \\
\lambda_{i}-\lambda_{i+1} & \min \left(\mu_{i}, \nu_{i}\right)-\lambda_{i}
\end{array}\right)
$$

It is now clear that $a=\min \left(\mu_{i-1}, \nu_{i-1}\right)-\lambda_{i-1}$, and one easily checks that for all $i \geq 0$, after completion of the exhaustion of $e_{k+i}^{\uparrow}$ and of the subsequent exhaustion of $e_{k+i+1}^{\uparrow}$, the submatrix $A_{\{k+i, k+i+1\} \times\{l-1, l\}}$ will have become

$$
\left(\begin{array}{cc}
\nu_{i}-\nu_{i+1} & a+\max \left(\mu_{i}-\nu_{i}, 0\right) \\
\nu_{i+1}-\nu_{i+2} & \min \left(\nu_{i}, \mu_{i}\right)-\lambda_{i}+\max \left(\mu_{i+1}-\nu_{i+1}, 0\right)
\end{array}\right) .
$$

Although $\max \left(\mu_{i+1}-\nu_{i+1}, 0\right)=\max \left(\mu_{i+1}, \nu_{i+1}\right)-\nu_{i+1}$ units have been added to the bottom right coefficient by the exhaustion of $e_{k+i+1}^{\uparrow}$, we do not expect that this reenables applications of $e_{k+i}^{\uparrow}$ (since such applications were not required for reducing the submatrix $A_{(\mathbf{N}-[k]) \times[l]}$ to $\mathrm{SF}(\nu, k, l)$ either $)$; indeed it follows from $\lambda_{i} \geq \max \left(\mu_{i+1}, \nu_{i+1}\right)$ that one has $\nu_{i}-\nu_{i+1} \geq \min \left(\nu_{i}, \mu_{i}\right)-\lambda_{i}+\max \left(\mu_{i+1}, \nu_{i+1}\right)-\nu_{i+1}$, whence further upward transfers are blocked. We may conclude that after the mentioned sequence of operations we will have determined the new value of column $l$ representing the composition $\kappa-\nu$.

In spite of the complications of the reduction, the resulting relation between $\lambda, \mu$, $\nu, \kappa$, and $A_{k, l}$ is surprisingly simple, as there is no "carry over" effect like there was in (39): the value of $a$ has no effect on the bottom-right entry in (52) that determines $\kappa_{i+1}-\nu_{i+1}$. Therefore we can describe the parts of $\kappa$ by the following explicit formulae rather than by an algorithm:

$$
\begin{aligned}
\kappa_{0} & =A_{k, l}+\max \left(\mu_{0}, \nu_{0}\right) \\
\kappa_{i+1} & =\min \left(\nu_{i}, \mu_{i}\right)-\lambda_{i}+\max \left(\mu_{i+1}, \nu_{i+1}\right) \quad \text { for all } i \in \mathbf{N} .
\end{aligned}
$$

Comparison with [vLee4, equation (15)] shows that this is the shape datum for the RSK correspondence, which reflects row-insertion on semistandard tableaux.

One can give the following interpretation of (53) in traditional terms. If one imagines, during RSK insertion, a semistandard Young tableau $S$ in whose display the entries $<l$ fill the diagram $[\lambda]$ while the entries $l$ fill $[\mu / \lambda]$, and if some insertion of 


\subsection{Other types of growth diagrams}

letters $<l$ into $S$ makes the shape filled by entries $<l$ grow to $[\nu]$, then upon subsequent row-insertion of $M_{k, l}$ letters $l$, these will be placed in row 0 starting at position $\max \left(\mu_{0}, \nu_{0}\right)$ (i.e., after the unbumped entries $l$ of $S$, if any of them remain, or else after the entries $<l$ present at that point) making the length of that row $\max \left(\mu_{0}, \nu_{0}\right)+A_{k, l}$. That insertion will not bump any letter $l$, but the previous insertion of letters $<l$ will bump $\min \left(\nu_{i}, \mu_{i}\right)-\lambda_{i}$ letters $l$ from row $i$ of $S$, and they will be placed in row $i+1$ starting at position $\max \left(\mu_{i+1}, \nu_{i+1}\right)$, making its length $\max \left(\mu_{i+1}, \nu_{i+1}\right)+\min \left(\nu_{i}, \mu_{i}\right)-\lambda_{i}$. Note in particular that it is the units that are not transferred upwards from the entry at $(i+k+1, l)$ in (51) that represent letters $l$ being bumped from row $i$ to row $i+1$.

Now that we have determined the shape datum, the family $\left(M_{(\mathbf{N}-[i]) \times[j]}\right)_{i, j \in \mathbf{N}}$ of shapes can be computed using a (vertically reversed) growth diagram, just like we have done before for other families of implicit shapes of submatrices. This time in fact we obtain a classical RSK growth diagram, growing from the bottom left corner of the matrix. We illustrate this in diagram 4 for our usual matrix $M$.

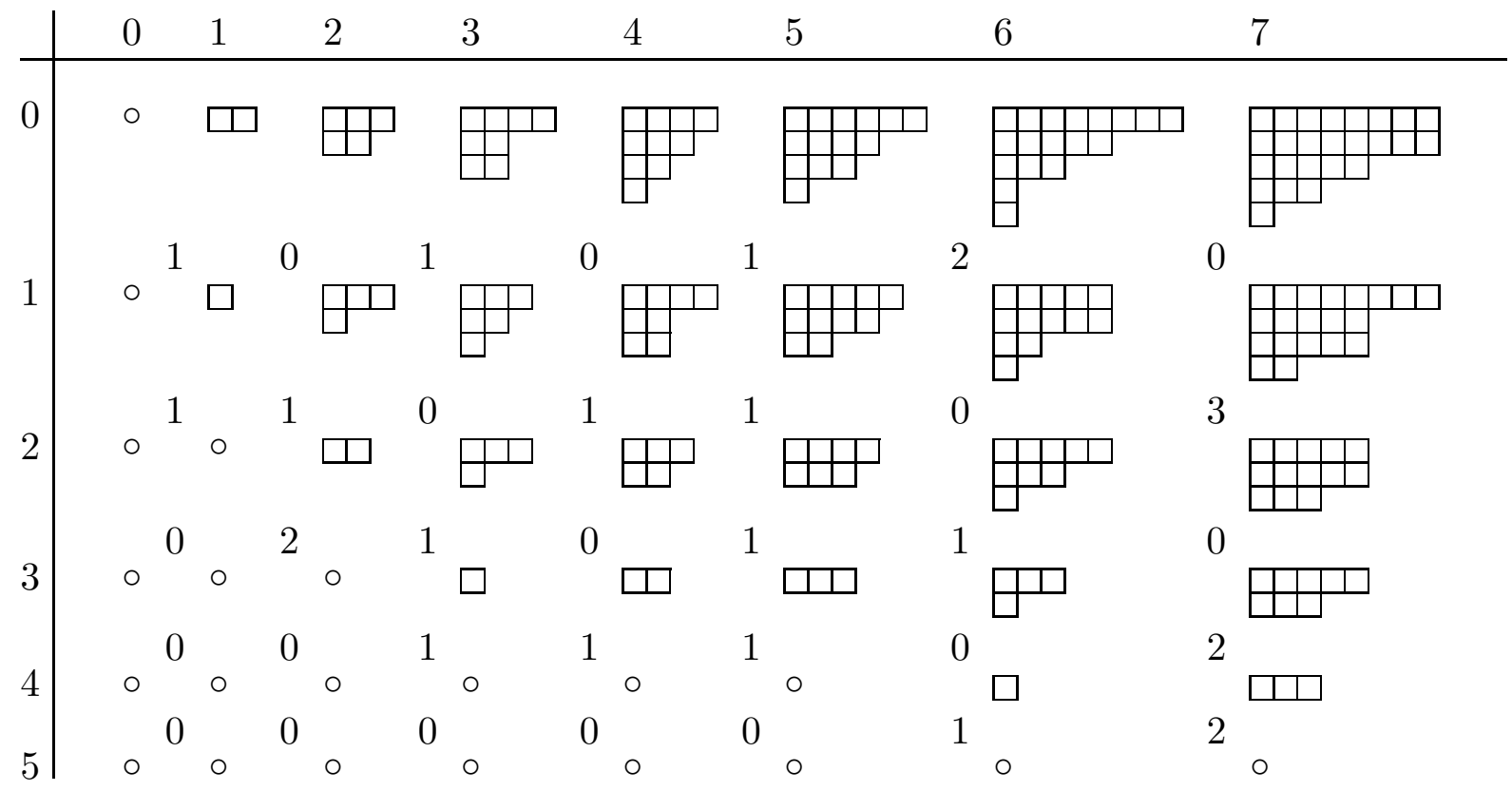

Diagram 4. Vertically reversed growth diagram with integral matrix and shapes $\left(\pi\left(M_{(\mathbf{N}-[i]) \times[j]}\right)\right)_{i, j \in \mathbf{N}}$, computed using the RSK shape datum.

4.4.4. Proposition. If for any $M \in \mathcal{M}$ one associates to each grid point $(i, j) \in \mathbf{N}^{2}$ the shape $\pi\left(M_{(\mathbf{N}-[i]) \times[j]}\right)$, then one obtains a vertically reversed growth diagram for the RSK shape datum.

Along the top border of diagram 4 we find the semistandard Young tableau $S$ of (31); this is no surprise since we already knew that $S=\left(\pi\left(M_{\mathbf{N} \times[j]}\right)\right)_{j \in \mathbf{N}}$, which was computed along the bottom border in diagram 1. Along the right border we find a reverse semistandard Young tableau $\left(\pi\left(M_{(\mathbf{N}-[i]) \times \mathbf{N}}\right)\right)_{i \in \mathbf{N}}$ that we did not encounter before. However, the way in which $S$, whose integral encoding is the matrix $P$ of $(29)$, 
was found here, corresponds to the description of theorem 3.3.1; according to comments following that theorem, the recording tableau of that insertion is the Schützenberger dual of the semistandard Young tableau $\bar{L}$ encoded by the matrix $Q^{t}$. Indeed the Schützenberger dual of

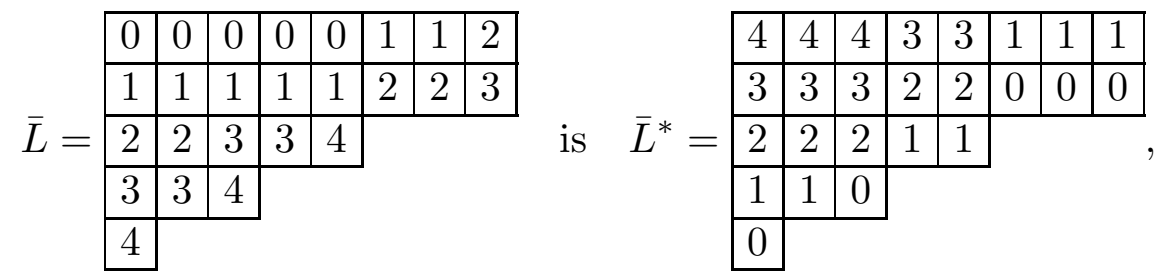

which is the reverse semistandard Young tableau that can be read off along the right border of diagram 4 .

\subsection{The Schützenberger involution.}

We have encountered the Schützenberger involution in two similar situations, for binary and for integral matrices. In the former case it defined a bijection mapping the reverse transpose semistandard Young tableau $R=\left(\pi\left(M_{\mathbf{N} \times(\mathbf{N}-[j]}\right)\right)_{j \in \mathbf{N}}$ to the transpose semistandard Young tableau $R^{*}=\left(\pi\left(M_{\mathbf{N} \times[j]}\right)\right)_{j \in \mathbf{N}}$; in the latter case it gave a bijection transforming the semistandard Young tableau $\bar{L}=\left(\pi\left(M_{[i] \times \mathbf{N}}\right)\right)_{i \in \mathbf{N}}$ into the reverse semistandard Young tableau $\bar{L}^{*}=\left(\pi\left(M_{(\mathbf{N}-[i]) \times \mathbf{N}}\right)\right)_{i \in \mathbf{N}}$. The fact that the members of these pairs of tableaux mutually determine each other, independently of $M$, may seem surprising at first glance, since at corresponding positions in their sequence they contain implicit shapes of complementary submatrices of $M$, which shapes are completely unrelated. On the other hand, these pairs of tableaux correspond to matrices obtained from $M$ by exhausting crystal operations in opposite directions. We have seen that $R$ and $R^{*}$ correspond to the matrices $P$ and $\widetilde{P}$ obtained from the binary matrix $M$ by exhausting upward respectively downward operations (cf. propositions 3.3.3 and 4.3.3). In the integral case $\bar{L}$ corresponds to the matrix $Q$ obtained from $M$ by exhausting leftward operations, and one easily sees that $\bar{L}^{*}$ similarly corresponds to a matrix $\widetilde{Q}$ obtained from $M$ by exhausting rightward operations (up to some sufficiently large limit); in the case of the example this is

$$
\widetilde{Q}=\left(\begin{array}{lllllll}
0 & 0 & 1 & 1 & 0 & 3 & 0 \\
0 & 0 & 0 & 2 & 2 & 0 & 3 \\
0 & 0 & 0 & 0 & 3 & 2 & 0 \\
0 & 0 & 0 & 0 & 0 & 3 & 2 \\
0 & 0 & 0 & 0 & 0 & 0 & 3
\end{array}\right)
$$

whose columns from right to left give the weights of the rows of the display of $\bar{L}^{*}$ from top to bottom. Since for each of the pairs of matrices $(P, \widetilde{P})$ and $(Q, \widetilde{Q})$ the members mutually determine each other (by exhaustion of appropriate crystal operations), it is clear that the same must be true for the pairs of tableaux $\left(R, R^{*}\right)$ and $\left(\bar{L}, \bar{L}^{*}\right)$. Exhaustion in the remaining pairs of opposite directions also give pairs of matrices that correspond to tableaux related by the Schützenberger involution. 


\subsection{The Schützenberger involution}

We shall not study the Schützenberger involution in detail here, and just observe some facts without formal proof. Its original definition in [Schü1] for standard Young tableaux can be generalised, using appropriate notions of standardisation, to semistandard or to transpose semistandard tableaux; doing so one must map tableaux of weight $\alpha$ either to tableaux of weight $\operatorname{rev}_{l}(\alpha)$ for some sufficiently large $l$, or to reversed tableaux of weight $\alpha$ (while keeping the semistandard or transpose semistandard attribute). Above we have implicitly assumed the latter, more elegant, form of these generalisations.

The original definition of the Schützenberger involution is by an algorithm that repeatedly applies a deflation operation (also called evacuation) to a standard Young tableau $S$, which removes a square from the diagram of its shape at every step, until the shape becomes (0); the resulting decreasing sequence of shapes defines the Schützenberger dual $S^{*}$ of $S$. It is natural to index the sequence defining the tableau $S^{(i)}$ deflated $i$ times by $\mathbf{N}-[i]$. If one then writes $S^{(i)}=\left(\lambda^{(i, j)}\right)_{j \in \mathbf{N}-[i]}$, then the definition of the deflation procedure amounts to a simple rule for determining $\lambda^{(i+1, j+1)}$ once $\lambda^{(i+1, j)}, \lambda^{(i, j)}$, and $\lambda^{(i, j+1)}$ are known. Thus $S=\left(\lambda^{(0, j)}\right)_{j=0}^{n}$ determines $S^{*}=\left(\lambda^{(i, n)}\right)_{i=0}^{n}$ via a two-parameter family of shapes governed by a local rule, in much the same way as a growth diagram, but without matrix entries.

The generalisations to semistandard or to transpose semistandard tableaux can be formulated in terms of deflation by an entire horizontal respectively vertical strip at a time, and give rise to similar families of shapes $\left(\lambda^{(i, j)}\right)_{i, j ; i \leq j}$, in which neighbouring shapes now differ by horizontal strips respectively by vertical strips. Here the rule for determining $\lambda^{(i+1, j+1)}$ given $\lambda^{(i+1, j)}, \lambda^{(i, j)}$, and $\lambda^{(i, j+1)}$ can be formulated as follows, using the notion of tableau switching for semistandard tableaux given in [vLee3, definition 2.2.4] (the transpose semistandard case is similar): applying tableau switching to the single-strip skew semistandard tableaux $T_{0}=\left(\lambda^{(i+1, j)}-\lambda^{(i, j)}\right)$ and $T_{1}=\left(\lambda^{(i, j)}-\right.$ $\left.\lambda^{(i, j+1)}\right)$ gives similar tableaux $\left(T_{1}^{\prime}, T_{0}^{\prime}\right)=X\left(T_{0}, T_{1}\right)$ where $T_{0}^{\prime}=\left(\lambda^{(i+1, j)}-\lambda^{(i+1, j+1)}\right)$ and $T_{1}^{\prime}=\left(\lambda^{(i+1, j+1)}-\lambda^{(i, j+1)}\right)$. An analysis of what tableau switching means in this particular case leads to the following alternative formulation: the two skew semistandard tableaux of two horizontal strips each, $\lambda^{(i+1, j)}-\lambda^{(i, j)}-\lambda^{(i, j+1)}$ and $\lambda^{(i+1, j)}-\lambda^{(i+1, j+1)}-\lambda^{(i, j+1)}$, are related to one another by a Bender-Knuth involution (the unique applicable one). Interestingly Bender-Knuth involutions, which we preferred to replace by ladder reversal involutions for establishing the symmetry of skew Schur functions to get more pleasant properties, thus still have their proper place in the theory.

The way in which we have encountered the Schützenberger involution in fact relates more naturally to a different way to compute it. The matrix $P$ obtained from $M \in \mathcal{M}$ by exhausting upward crystal operations as in (29) encodes a unique semistandard Young tableau $S$, of shape $\pi /(0)$ where $\pi=\pi(M)$. If $k>0$ is so large that $M=M_{[k] \times \mathbf{N}}$, then the matrix $\widetilde{P}$ obtained from $M$ by exhausting downward crystal operations $f_{i}^{\downarrow}$ for $i \in[k-1]$ determines the Schützenberger dual $S^{*}=\left(\mu^{(j)}\right)_{j \in \mathbf{N}}$ of $S$, namely by $\mu^{(j)}=$ $\pi\left(\widetilde{P}_{\mathbf{N} \times(\mathbf{N}-[j])}\right)=\operatorname{rev}_{k}\left(\operatorname{row}\left(\widetilde{P}_{\mathbf{N} \times(\mathbf{N}-[j])}\right)\right)$. But if one fixes $l \geq \pi_{0}$, and $\rho \in \mathcal{P}$ is such that $[\rho]=[k] \times[l]$, then $\widetilde{P}$ is also the integral encoding of a unique semistandard tableau of 
shape $\rho / \pi^{\diamond}$, where $\pi^{\diamond}=\rho-\operatorname{rev}_{k}(\pi)$, namely of $S^{* \diamond}=\left(\mu^{(j) \diamond}\right)_{j \in \mathbf{N}}=\left(\rho-\operatorname{rev}_{k}\left(\mu^{(j)}\right)\right)_{j \in \mathbf{N}}$. This is the tableau whose display is obtained from that of $S^{*}$ by the rotation by a half turn that maps the rectangle $[\rho]$ to itself.

The semistandard tableaux $S$ and $S^{* \diamond}$ are encoded by integral matrices $P$ and $\widetilde{P}$ that can be obtained from one another by exhausting upward respectively downward crystal operations, so it follows from proposition 2.5 that $S$ is the rectification by jeu de taquin of $S^{* \diamond}$, and since rotation by a half turn transforms inward jeu de taquin slides into outward slides, $S^{* \diamond}$ is similarly the "bottom-right rectification" of $S$ within the rectangular shape $\rho$. In our example one may check, for $(k, l)=(5,8)$, that

$$
\widetilde{P}=\left(\begin{array}{lllllll}
1 & 0 & 0 & 0 & 0 & 0 & 0 \\
1 & 1 & 1 & 0 & 0 & 0 & 0 \\
0 & 2 & 1 & 1 & 1 & 0 & 0 \\
0 & 0 & 1 & 1 & 3 & 3 & 0 \\
0 & 0 & 0 & 0 & 0 & 1 & 7
\end{array}\right) \quad \text { and } \quad S^{* \diamond}=\begin{array}{ll|l|l|l|l|l|l|}
n & & 0 & 1 & 1 & 2 \\
\hline 2 & 3 & 4 & 4 & 4 & 5 & 5 & 5 \\
\hline 5 & 6 & 6 & 6 & 6 & 6 & 6 & 6 \\
\hline
\end{array}
$$

and that the rectification of $S^{* \diamond}$ is the tableau $S$ of (31); thus $S \leftrightarrow S^{*}$ can be computed via rectification. The same relation exists between $\bar{L}$ and $\bar{L}^{* \diamond}$ in (54), and between $R$ in (35) and $R^{* \diamond}$ obtained from $R^{*}$ in (45); in the latter case a version of jeu de taquin for transpose semistandard tableaux is used.

This alternative method of computing the involution was first presented by Schützenberger in [Schü2]. It is not so easy to deduce it from the original definition of the Schützenberger involution, but the opposite is straightforward, once it is established that the rectification of any semistandard tableau $T$ of shape $\rho / \lambda^{\diamond}$ is of shape $\lambda$. Then the shape $\mu^{(j)}$ in $S^{*}=\left(\mu^{(j)}\right)_{j \in \mathbf{N}}$ can be determined as the shape of the rectification of the skew tableau $S^{(j)}$ obtained by removing $j$ initial horizontal strips from $S$, and these shapes can be found by successively deflating $S$ by one horizontal strip at a time. By construction that rectification is given by the sequence of shapes $\left(\lambda^{(j, l)}\right)_{l=j}^{n}$, where $\left(\lambda^{(j, l)}\right)_{j, l ; j \leq l \leq n}$ is the semistandard tableau switching family used to compute $S^{*}=\left(\lambda^{(j, n)}\right)_{j=0}^{n}$ starting from $S=\left(\lambda^{(0, l)}\right)_{l=0}^{n}$.

Now if $P$ is the integral encoding of $S$, then $P_{\mathbf{N} \times(\mathbf{N}-[j])}$ is the integral encoding of $S^{(j)}$, for all $j \in \mathbf{N}$, and the integral encoding of the rectificationof $S^{(j)}$ can be found by exhausting upwards crystal operations applied to $P_{\mathbf{N} \times(\mathbf{N}-[j])}$. This gives have the following interpretation for the shapes in the semistandard tableau switching family: $\lambda^{(j, l)}=\pi\left(M_{\mathbf{N} \times([l]-[j])}\right)$, which agrees with $S^{*}=\left(\pi\left(P_{\mathbf{N} \times(\mathbf{N}-[j])}\right)\right)_{j \in \mathbf{N}}$. Since vertical crystal operations do not change the implicit shape, $\left(\pi\left(M_{\mathbf{N} \times([l]-[j])}\right)\right)_{j, l ; j \leq l}$ is a semistandard tableau switching family for any integral matrix $M$. The same is true for $\left(\pi\left(M_{([k]-[i]) \times \mathbf{N}}\right)\right)_{i, k ; i \leq k}$; these statements hold in the binary case as well, with the adjustment that the former family is transpose semistandard. However, in contrast with the situation for the various growth diagrams we considered, we have not been able to find a simple direct argument why a local rule for these families of shapes should exist, without referring to the entire family; for instance one showing that knowledge of the shapes $\pi\left(M_{\mathbf{N} \times([l]-[j+1])}\right), \pi\left(M_{\mathbf{N} \times([l]-[j])}\right)$, and $\pi\left(M_{\mathbf{N} \times([l+1]-[j])}\right)$ suffices to determine $\pi\left(M_{\mathbf{N} \times([l+1]-[j+1])}\right)$. In principle one could establish the local rule for that family using 
those for $\left(M_{[k] \times[l]}\right)_{k, l \in \mathbf{N}}$ and for $\left(M_{[k] \times(\mathbf{N}-[j])}\right)_{k, j \in \mathbf{N}}$, and induction on $k$, similarly to the proof of [vLee1, theorem 4.1.1], but this is not very attractive.

\section{$\S 5$. Characterisation of the implicit shape.}

The implicit shape of a matrix $M$ characterises by definition the double crystal in which $M$ occurs. We have by now seen many different ways to compute this shape, but these involve either transforming $M$ into a more special matrix within its double crystal (by exhausting some operations), or constructing a growth diagram from $M$. It would be convenient to have a characterisation of $\pi(M)$ in terms of $M$ itself, which would provide a quantity invariant on double crystals and distinguishing any two distinct double crystals. In this section we shall present such a characterisation of $\pi(M)$, which will however usually not provide a practical way to compute it; the methods given above can therefore be interpreted as algorithms to compute the invariant that we shall describe below.

It is well known that the length of the longest increasing and decreasing sequences extracted from a permutation $\sigma$ (represented as a word) are given by the lengths of the top row and leftmost column of the tableaux associated to $\sigma$ under the Schensted correspondence; extending this result, it was shown in [Gre1] that the entire common shape of those tableaux can be characterised in terms of the permutation matrix (which is viewed as describing a partially ordered set). A version of Greene's poset invariant can also be defined for integral and binary matrices, with a similar interpretation as the shape of the tableaux associated under the RSK correspondence and its dual. Therefore we shall not waste any time trying to derive the invariant we are looking for, and simply present the proper formulation. (Note that the word invariant is used in two different senses here: the partition defined by Greene depends only on the abstract finite poset defined by the permutation, but its extension to matrices is invariant under crystal operations, even though these do in general alter the associated poset.)

5.1. Definitions. Let $M \in \mathcal{M}^{[2]}$ be a binary matrix and $N \in \mathcal{M}$ an integral matrix, and $m \in \mathbf{N}$.

a. $M$ is called a chain if $M_{k, l}=1$ implies $M_{i, j}=0$ for all $i<k$ and $j \geq l$, and it is called an antichain if $M_{k, l}=1$ implies $M_{i, j}=0$ for all $i \leq k$ and $j<l$.

b. $N$ is called a chain if $N_{k, l}>0$ implies $N_{i, j}=0$ for all $i<k$ and $j<l$, and it is called an antichain if $N_{k, l} \leq 1$ for all $k, l \in \mathbf{N}$, while $N_{k, l}=1$ implies $N_{i, j}=0$ for all $(i, j) \neq(k, l)$ with $i \leq k$ and $j \geq l$.

c. An $m$-chain or an $m$-antichain in $\bar{M}$ is a sequence $\left(M^{(k)}\right)_{k \in[m]}$ of $m$ binary matrices, all chains respectively antichains, with $M=\sum_{k \in[m]} M^{(k)}+M^{\prime}$ for some $M^{\prime} \in \mathcal{M}^{[2]}$.

d. An $m$-chain or an $m$-antichain in $N$ is a sequence $\left(N^{(k)}\right)_{k \in[m]}$ of $m$ integral matrices, all chains respectively antichains, with $N=\sum_{k \in[m]} N^{(k)}+N^{\prime}$ for some $N^{\prime} \in \mathcal{M}$.

e. The m-length of a binary or integral matrix $A$ is the maximum of $\sum_{k \in[m]}\left|A^{(k)}\right|$ taken over all $m$-chains $\left(A^{(k)}\right)_{k \in[m]}$ in $A$, where $|A|=\sum_{i, j \in \mathbf{N}} A_{i, j}$. The $m$-breadth of $A$ is the maximum of $\sum_{k \in[m]}\left|A^{(k)}\right|$ taken over all $m$-antichains $\left(A^{(k)}\right)_{k \in[m]}$ in $A$.

The terminology is derived from the partially ordered sets associated as follows to 
the matrices. For $M \in \mathcal{M}^{[2]}$ an element $x_{i, j}$ is associated to every bit $M_{i, j}=1$, and $x_{i, j}<x_{k, l}$ holds if and only if $i \leq k$ and $j<l$. For $N \in \mathcal{M}$ a chain of $N_{i, j}$ elements is associated to every entry $N_{i, j}$, and for every element $x$ of the chain for $N_{i, j}$ and every element $y$ of the chain for another entry $N_{k, l}$ one has $x<y$ if and only if $i \geq k$ and $j \leq l$. In this setting it is convenient to use some more related terminology. For an $m$-chain or $m$-antichain $\left(A^{(k)}\right)_{k \in[m]}$, either binary or integral, we shall call $\sum_{k \in[m]}\left|A^{(k)}\right|$ its size. If $A, B$ are binary or integral matrices, the matrix $\left(\min \left(A_{i, j}, B_{i, j}\right)\right)_{i, j \in \mathbf{N}}$ will be called their intersection $A \cap B$; in case $B=A \cap B$ we shall write $A \supseteq B$ and say that $A$ contains $B$. In particular $A$ contains the sum of any $m$-(anti)chain in $A$. Then the intersection of a chain and an antichain has size at most 1, and it follows that the sum of an $m$-chain cannot contain any antichain of size $>m$, and that the sum of an $m$ antichain cannot contain any chain of size $>m$. The converse is also true, namely that any matrix that does not contain any antichains of size $>m$ is the sum of an $m$-chain, and one that does not contain any chains of size $>m$ is the sum of an $m$-antichain, but this is somewhat less obvious.

The definition is formulated so as to emphasise the similarities between the different cases, but there are notable differences as well. In the binary case chains and antichains can be interpreted as sets of squares where the matrix has bits ' 1 ', and the sums involved in $m$-(anti)chains obviously must correspond to disjoint unions of such sets. In the integral case antichains can also be seen as sets of squares, but the antichains in an $m$-antichain need not be disjoint, or even distinct. On the other hand one could require in the definition of $m$-length for the integral case that the nonzero entries in the chains of the $m$-chain involved occur in disjoint squares: the indicated maximum would not decrease due to this restriction.

It is not hard to check both in the binary and in the integral case that a matrix $M$ is a chain if and only if $\pi(M)$ has at most one non-zero part, in other words if $\pi(M)_{1}=0$, and that $M$ is an antichain if and only if all parts of $\pi(M)$ are $\leq 1$, in other words if $\pi(M)_{1}^{t}=0$. In general it is quite hard to determine, for a given $M$ and $m$, an $m$ (anti)chain in $M$ of maximal size. In the special cases $M=\operatorname{Diag}^{[2]}(\lambda)$ and $M=\operatorname{Diag}(\lambda)$ however, this is easy. For $M=\operatorname{Diag}^{[2]}(\lambda)$, the rows of $M$ define chains and the columns antichains, and the $m$-chain or $m$-antichain formed by the $m$ longest rows respectively columns has the maximal possible size, since its intersection with each individual column respectively row has maximal size. For $M=\operatorname{Diag}(\lambda)$, each chain contained in $M$ has at most one nonzero (diagonal) entry, so an $m$-chain of maximal size contained in $M$ is formed by extracting the $m$ largest diagonal entries: $\left(M_{[i] \times[i]}\right)_{i \in[m]}$. In this case the size of an $m$-antichain in $M$ is at most $\sum_{i \in \mathbf{N}} \min \left(m, M_{i, i}\right)$, and this size is obtained by taking the $m$-antichain $\left(\mathbf{1}\left(\lambda_{j}^{t}\right)\right)_{j \in[m]}$, where $\mathbf{1}(l)$ denotes the $l \times l$ identity matrix $([i=j \in[l]])_{i, j \in \mathbf{N}}$. Note that in both cases the $m$-length of $M$ is $\sum_{i \in[m]} \lambda_{i}$, and its $m$-breadth is $\sum_{i \in[m]} \lambda_{i}^{t}$. The main result of this subsection is that this is true in general, where $\lambda=\pi(M)$.

5.2. Theorem. For any binary or integral matrix $M$, and any $m \in \mathbf{N}$, the $m$-length of $M$ is equal to $\sum_{i \in[m]} \lambda_{i}$, and the m-breadth of $M$ is equal to $\sum_{i \in[m]} \lambda_{i}^{t}$, where $\lambda=\pi(M)$. 


\section{Characterisation of the implicit shape}

Since we know the theorem holds for normal forms, it is equivalent to the statement that for every $m$ the $m$-length and $m$-breadth of a matrix are invariant under all crystal operations, and this is the way in which we shall prove it. Note that one consequence of the theorem is that the sequence of all $m$-lengths of $M$ determines that of all $m$ breadths and vice versa. It is known that this statement is true in general for finite posets, see [Gre2, Theorem 1.6], but a proof for that case is rather more difficult than for the situation of our theorem.

There are numerous ways to approach a proof of the theorem, many of which start by reducing to a simplified situation. It is fairly easy to see that the argument by which the theorem holds for normal forms can be adapted to show that is holds whenever either upward or leftward moves are exhausted, so that a proof for operations in one direction would suffice (but this facilitates only the binary case). The result is also known to hold for permutation matrices, see [Gre1, Theorem 3.1], and it is possible to enlarge matrices, pulling their units apart so as to obtain a permutation matrix, while not changing their implicit shape or $m$-length and $m$-breadth; this would provide a proof. One can define a notion of chains in the display of a semistandard tableau $T$, and [Schü2, théorème 3.1] states a variant of Greene's result for that case, where the shape is that of the rectification of $T$; it can be seen that the $m$-length and $m$-breadth so associated to $T$ are the same as those of its integral encoding, so this would provide a more direct proof for the integral case (but since the chains of $T$ do not correspond to those of its binary encoding, more work would be needed for that case). Finally one could try, as is done in the proofs of the results cited, to decompose the changes to the poset structure into minimal modifications involving only three elements, and prove that these do not effect $m$-length or $m$-breadth.

All of these methods however require arguments to justify the reduction, which are not related directly to the definitions of $m$-length or $m$-breadth, or of crystal operations; they also depend on nontrivial results about the Robinson-Schensted correspondence. We believe that for understanding why the theorem is true, it is most instructive to use a head-on approach, showing directly that the definition of crystal operations prevents them from changing any $m$-length or $m$-breadth; in fact this is not too hard to do, although the situation is more complicated than in the 3-unit case. By using only the definition of crystal operations, this approach could even serve as a starting point for developing their theory (but we believe this would not would make that development more transparent). We also choose a "positive" point of view, characterising matrices that are the sum of an $m$-(anti)chain systematically by the existence of such a decomposition, rather than by the non-existence of any antichain (respectively chain) of size $m+1$. The proof we give will consist of a series of lemmas that each prove a part of the mentioned invariance.

The number of cases to be treated in principle is considerable, since we need to show both in the binary and the integral case that neither the $m$-length nor the $m$-breadth can increase nor decrease under crystal operations in any of the four possible directions. We reduce this number using the usual symmetry considerations to four distinct cases, which can moreover be regarded as two pairs of analogous cases. Both in the binary and integral cases, rotation by a half turn leaves both $m$-length and $m$-breadth invariant, so 
for each of these quantities and any pair of opposite directions it will suffice to show that the quantity will not decrease under a crystal operation in one direction: by rotation symmetry the inverse crystal operation will then not decrease the quantity either, so that it is invariant under both operations. In the binary case, rotation of matrices by a quarter turn transforms $m$-chains into $m$-antichains, which permits us to consider only the $m$-length; in the integral case we must consider both $m$-length and $m$-breadth, but transposition symmetry will allow us to consider transfers in one direction only.

The binary and the integral case are sufficiently different to merit separate treatment. We shall start with the somewhat simpler binary case, where as said we shall consider only the $m$-chains. Much if our discussion will be about locating bits $M_{i, j}=1$; we shall call this a unit at position $(i, j)$, and we shall view the individual chains of an $m$-chain in $M$ as sets of such units.

5.3. Lemma. Let a binary matrix $M \in \mathcal{M}^{[2]}$ contain an $m$-chain of size $n$ for some $m, n \in \mathbf{N}$, and let $M^{\prime}=e_{k}^{\uparrow}(M)$ for some $k \in \mathbf{N}$. Then $M^{\prime}$ also contains an $m$-chain of size $n$.

Proof. Let $M_{k, l}=0$ and $M_{k+1, l}=1$ be the bits interchanged by the application of $e_{k}^{\uparrow}$. One may assume that the unit at $(k+1, l)$ occurs in one of the chains $C$ of the $m$ chain, for otherwise one can simply keep the same $m$-chain. If, after removing the unit at $(k+1, l)$ from $C$, any of the chains of the $m$-chain would remain a chain when a unit at $(k, l)$ is added to it, then an $m$-chain of size $n$ in $M^{\prime}$ can be formed in that way; we shall show that after possibly changing the choice of the $m$-chain of size $n$ in $M$, we can always arrange to be in that situation. Let the units of $C$ to the left of $(k+1, l)$ in row $k+1$ be at positions $\left\{\left(k+1, j_{i}\right) \mid i \in[r]\right\}$ where $r \in \mathbf{N}$ and $j_{0}<\cdots<j_{r-1}<l$. If $r=0$ (i.e., if there are no such units) then after removing the unit at $(k+1, l)$ we can add a unit at $(k, l)$ to $C$ itself, and we are done.

Otherwise definition 1.3.1a gives us $\sum_{j=j_{0}}^{l-1} M_{k, j} \geq \sum_{j=j_{0}}^{l-1} M_{k+1, j} \geq r$, so there are at least $r$ different indices $j<l$ such that $M_{k, j}=1$; let the $r$ largest such indices be $j_{0}^{\prime}<\cdots<j_{r-1}^{\prime}$. From the fact that $\sum_{j=j_{i}}^{l-1} M_{k, j} \geq \sum_{j=j_{i}}^{l-1} M_{k+1, j}$ for $i \in[r]$, it follows that $j_{i} \leq j_{i}^{\prime}$ for all $i$. None of the units $M_{k, j_{i}^{\prime}}=1$ can occur in $C$ because of its unit at $\left(k+1, j_{0}\right)$. If none of them occur in any of the other chains of the $m$-chain either, then one can modify $C$ by removing the units at $\left(k+1, j_{i}\right)$ and adding units at $\left(k, j_{i}^{\prime}\right)$, for $i \in[r]$; this reduces us to the case $r=0$ already treated. If at least one of the units $M_{k, j_{i}^{\prime}}=1$ does occur in a chain of the $m$-chain, let $j_{s}^{\prime}$ be the maximal index for which this happens, and let the unit at $\left(k, j_{s}^{\prime}\right)$ occur in the chain $C^{\prime}$. If $C^{\prime}$ contains any units in row $k$ to the right of the one at $\left(k, j_{s}^{\prime}\right)$ then this must be in a column $>l$, and a unit at $(k+1, l)$ can be added to $C^{\prime}$. If not, then we replace $C, C^{\prime}$ as follows by a new pair of chains $D, D^{\prime}$ of the same total size: the chain $D$ contains the units of $C^{\prime}$ in columns $\leq j_{s}^{\prime}$, the units at $\left(k, j_{i}^{\prime}\right)$ for $s<i<r$ and the units of $C$ in columns $\geq l$, while $D$ contains the units of $C$ in columns $\leq j_{s}$ and the units of $C^{\prime}$ in columns $>j_{s}^{\prime}$. After this change $D$ takes over the role of $C$, and we are in the case $r=0$, so we are done.

This situation of the final case of the proof is schematically represented in figure 1 , in which black circles represent units, the white circle is the bit $M_{k, l}=0$, the red 
and blue lines indicate the chains $C$ and $C^{\prime}$, with dotted parts that do not occur in $D$ with the unit at $(k+1, l)$ replaced by one at $(k, l)$, nor in $D^{\prime}$; the green lines indicate the new parts of the latter chains and the yellow dotted lines show the correspondence $\left(k+1, j_{i}\right) \leftrightarrow\left(k, j_{i}^{\prime}\right)$.

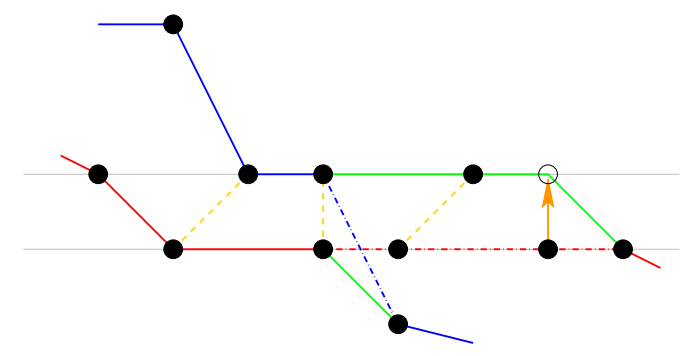

Figure 1. Schematic view of an partial exchange of chains for a vertical move in a binary matrix.

5.4. Lemma. Let a binary matrix $M \in \mathcal{M}^{[2]}$ contain an $m$-chain of size $n$ for some $m, n \in \mathbf{N}$, and let $M^{\prime}=e_{l}^{\leftarrow}(M)$ for some $l \in \mathbf{N}$. Then $M^{\prime}$ also contains an $m$-chain of size $n$.

Before stating the somewhat technical formal proof, we shall give an informal reasoning leading to it, ordering the cases that can occur by increasing complication. Let $M_{k, l}=0$ and $M_{k, l+1}=1$ be the bits interchanged by $e_{l}^{\leftarrow}$. First consider the relatively simple case $m=1$. If the unit at $(k, l+1)$ does not occur in the given (1-)chain $C$, then $C$ will still be a chain in $M^{\prime}$. If the unit does occur in $C$, we can just replace it in $C$ by the unit that was moved to the left, unless $C$ already contains a unit in column $l$, necessarily at some $\left(k^{\prime}, l\right)$ with $k^{\prime}<k$. The inequality $\sum_{i=k^{\prime}}^{k-1} M_{i, l} \leq \sum_{i=k^{\prime}}^{k-1} M_{i, l+1}$ from definition $1.3 .1 \mathrm{~b}$ shows that there exists a unit at $\left(k^{\prime \prime}, l+1\right)$ with $k^{\prime} \leq k^{\prime \prime}<k$; then by replacing in $C$ the unit at $(k, l+1)$ by the one at $\left(k^{\prime \prime}, l+1\right)$, one obtains a chain of size $n$ in $M$ that is also in $M^{\prime}$.

In the case $m>1$ there is at most one chain $C$ in the $m$-chain with a unit at $(k, l+1)$, and if so one can apply the reasoning above to $C$, in order to find another chain to replace it in the $m$-chain. The reasoning proceeds as before, but at the end there is a complication in case the unit at $\left(k^{\prime \prime}, l+1\right)$ occurs in another chain $C^{\prime}$ of the $m$-chain. If $C^{\prime}$ contains no unit in column $l$, then one can resolve the difficulty by "genetic recombination" of $C$ and $C^{\prime}$ (a crossover of chains somewhat simpler than the one in figure 1): the unit at $\left(k^{\prime}, l\right)$ and any other units of $C$ in columns $<l$ combine with the unit at $\left(k^{\prime \prime}, l+1\right)$ and any other units of $C^{\prime}$ in columns $>l+1$ to form a new chain $D$, while the remaining units of $C^{\prime}$, which are in columns $<l$, combine with the unit at $(k, l+1)$ and any other units of $C$ in columns $>l+1$, to form another new chain $D^{\prime}$, which does not contain a unit in column $l$. This situation is schematically represented in figure 2, using the same conventions as in figure 1. If on the other hand $C^{\prime}$ does contain a unit in column $l$, then one can repeat the same kind of argument to find another unit in column $l+1$, and so on; eventually there must be such a unit that is not in a chain with another unit in column $l$, and all the pieces of the chains involved can be pieced together to form an $m$-chain in $M^{\prime}$ (unlike for the previous lemma, any 
number of chains can be involved in the modifications here). In order to proceed in an orderly fashion the formal proof shall start with untangling any chains that might be involved. Figure 3 schematically shows the two types of modification that may be required at the end of the proof below to reorganise the chains in the most difficult cases: a shift by several units in column $l+1$ between chains, or a cyclic recombination of parts of several chains.

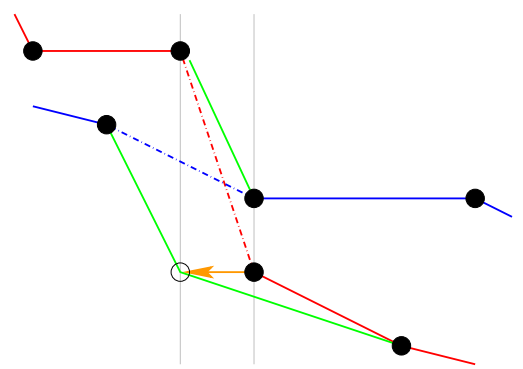

Figure 2. Schematic view of a simple recombination of chains for a horizontal move in a binary matrix.

Proof. Let the move effected by $e_{j}^{\leftarrow}$ interchange bits $M_{k, l}=0$ and $M_{k, l+1}=1$. Consider the subset $S$ of chains in the $m$-chain that have a unit both in column $l$ and in column $l+$ 1 , and put $r=\# S \geq 0$. Let $I$ be the set of rows of those units in column $l$, and $I^{\prime}$ the set of rows of those units in column $l+1$. Since each chain of $S$ contributes an $i \in I$ and an $i^{\prime} \in I^{\prime}$ with $i \leq i^{\prime}$, it follows after sorting that if $I=\left\{i_{0}, \ldots, i_{r-1}\right\}$ and $I^{\prime}=\left\{i_{0}^{\prime}, \ldots, i_{r-1}^{\prime}\right\}$ with $i_{0}<\cdots<i_{r-1}$ and $i_{0}^{\prime}<\cdots<i_{r-1}^{\prime}$, then $i_{j} \leq i_{j}^{\prime}$ for all $j \in[r]$. Then we may assume (after recombining parts of the chains if necessary) that the units $M_{i_{j}, l}=1$ and $M_{i_{j}^{\prime}, l+1}=1$ occur in the same chain $C^{(j)} \in S$, for all $t \in[r]$.

If $k \notin I^{\prime}$, then either none of the chains of the $m$-chain has a unit at $(k, l+1)$ so that one can keep the $m$-chain, or that unit occurs in a chain without unit in column $l$ so that it can be replaced in that chain by a unit at $(k, l)$. Therefore assume that $k=i_{t}^{\prime}$ for some $t \in[r]$, so that the chain $C^{(t)}$ contains units at $(k, l+1)$ and at $\left(i_{t}, l\right)$; since $M_{k, l}=0$ one must have $i_{t}<k$. Now for any $j \leq t$, definition 1.3.1b gives $\sum_{i=i_{j}}^{k-1} M_{i, l} \leq \sum_{i=i_{j}}^{k-1} M_{i, l+1}$, and each of the chains $C^{(j)}, \ldots, C^{(t-1)}$ contributes equally to both sides, while $C^{(t)}$ contributes only to the left hand side. Then the right hand side either has a contribution not from any chain in $S$, or one from $C^{(j-1)}$. The latter does not happen for $j=0$, so there exists some $i<k$ with $M_{i, l+1}=1$ and $i \notin I^{\prime}$; now put $k^{-}=\max \left\{i<k \mid M_{i, l+1}=1, i \notin I^{\prime}\right\}$ and $s=\min \left\{j \leq t \mid i_{j}^{\prime}>k^{-}\right\} \leq t$. The argument given shows that $i_{s} \leq k^{-}$, and that $i_{j} \leq i_{j-1}^{\prime}$ for $s<j \leq t$.

Now if the unit at $\left(k^{-}, l+1\right)$ does not occur in any chain of the $m$-chain, then one can replace in $C^{(s)}$ the unit at $\left(i_{s}^{\prime}, l+1\right)$ by one at $\left(k^{-}, l+1\right)$, and for $s<j \leq t$ replace in $C^{(j)}$ the unit at $\left(i_{j}^{\prime}, l+1\right)$ by one at $\left(i_{j-1}^{\prime}, l+1\right)$; the $m$-chain of size $n$ in $M$ so obtained is also an $m$-chain in $M^{\prime}$. Finally if some chain of the $m$-chain does contain a unit at $\left(k^{-}, l+1\right)$, the this chain does not lie in $S$, and one can "cyclicly recombine" that chain $C$ with the chains $C^{(j)}$ for $s \leq j \leq t$ : any units of $C$ in columns $<l$, are combined with the unit at $(k, l+1)$ and any other units of $C^{(t)}$ in columns $>l$ 
to form a new chain $D$; the units in columns $\leq l$ of $C^{(s)}$ are combined with the unit at $\left(k^{-}, l+1\right)$ and any other units of $C$ in columns $>l$; and for $s<j \leq t$ the units in columns $\leq l$ of $C^{(j)}$ are combined with the units in columns $>l+1$ of $C^{(j-1)}$. We have obtained an $m$-chain in $M$ of size $n$, in which the chain $D$ contains a unit at $(k, l+1)$ that can be replaced by a unit at $(k, l)$ to form an $m$-chain in $M^{\prime}$ of size $n$.
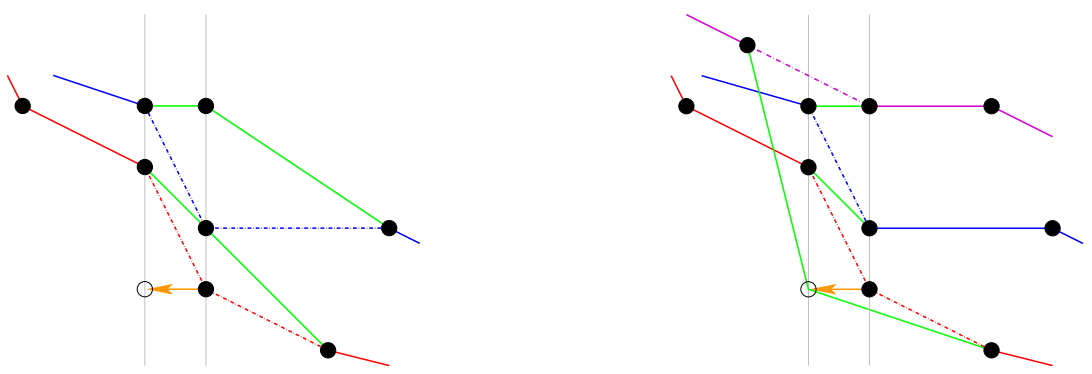

Figure 3. Schematic view of a shift between chains by several units, and of a cyclic recombination of chains, for a horizontal move in a binary matrix.

We now consider the integral case, where the cases of $m$-chains and $m$-antichains must be treated separately, but moves in only one direction need to be considered. As we shall see, these two cases are like "thick" versions of those treated in lemmas 5.3 and 5.4, respectively. The thickness refers to the fact that many units can share a same position in the matrix; this seems to be a slight advantage in the case of chains (which are now more compact), but a disadvantage in the case of antichains (more effort is needed to tell them apart). To emphasise the indicated analogy, we shall deal with upward transfers for $m$-chains, and with leftward transfers for $m$-antichains.

5.5. Lemma. Let an integral matrix $M \in \mathcal{M}$ contain an $m$-chain of size $n$ for some $m, n \in \mathbf{N}$, and let $M^{\prime}=e_{k}^{\uparrow}(M)$ for some $k \in \mathbf{N}$. Then $M^{\prime}$ contains an $m$-chain of size at least $n$.

Proof. Let the transfer effected by $e_{k}^{\uparrow}$ be from $M_{k+1, l}$ to $M_{k, l}$. One may assume that one of the chains $C$ of the $m$-chain contains a unit at $(k+1, l)$, for otherwise one can simply keep the same $m$-chain. If any chain of the $m$-chain can accommodate a (new) unit at $(k, l)$ while remaining a chain, then an $m$-chain of size $n$ in $M^{\prime}$ can simply be constructed by removing a unit from $C$ at $(k, l+1)$ and adding a unit at $(k, l)$ to the chain in question. We shall show that one can always reach this situation by replacing if necessary the given $m$-chain in $M$ by another one of size at least $n$.

Let $l^{\prime}=\max \left\{j \mid C_{k+1, j}>0\right\} \geq l$ be the column of the rightmost unit of $C$ in row $k+1$. If $l^{\prime}=l$ then $C$ itself can accommodate a unit at $(k, l)$. Otherwise, if none of the chains in the $m$-chain have any units at $(k, j)$ for $l \leq j<l^{\prime}$ then we may replace $C$ by a chain obtained by removing all its units at positions $(k+1, j+1)$ with $l \leq j<l^{\prime}$, while adding for those same values of $j$ all $M_{k, j}$ units of $M$ at $(k, j)$. The inequality $\sum_{j=l}^{l^{\prime}-1}\left(M_{k, j}-M_{k+1, j+1}\right) \geq 0$ from definition 1.4.1a shows that the size of the new chain is at least that of $C$, so we have reduced to the case $l^{\prime}=l$. If some of the chains does have a unit at some $(k, j)$ with $l \leq j<l^{\prime}$, then let $j_{0}$ be the minimal such index $j$, 
and $C^{\prime}$ a chain of our $m$-chain with a unit at $\left(k, j_{0}\right)$. If $C^{\prime}$ has another unit in row $k$ to the left of the one at $\left(k, j_{0}\right)$, necessarily in a column $<l$, then it can accommodate an additional unit at $(k, l)$. If $C^{\prime}$ has no such unit, then we can replace the chains $C, C^{\prime}$ by a pair of new chains $D, D^{\prime}$ formed as follows: the chain $D$ contains the units of $C$ in columns $\leq l$, all $M_{k, j}$ units available in $M$ at $(k, j)$ for $l \leq j<j_{0}$, and the units of $C^{\prime}$ in rows $\leq k$, which are in columns $\geq j_{0}$; the chain $D^{\prime}$ contains the units of $C^{\prime}$ in rows $>k$, which are in columns $\leq j_{0}$, and the units of $C$ in columns $>j_{0}$. The inequality $\sum_{j=l}^{j_{0}-1}\left(M_{k, j}-M_{k+1, j+1}\right) \geq 0$ shows that replacing $C, C^{\prime}$ by $D, D^{\prime}$ gives an $m$-chain of size at least $n$, and $D$ can accommodate an additional unit at $(k, l)$.

We note that in the final case we could have included any units of $C$ at $\left(k+1, j_{0}\right)$ into $D^{\prime}$, but the $m$-chain will be large enough without them; in fact we could have done without the units of $C^{\prime}$ at that position as well. But there need not be any such units, and there will not be if $n$ is maximal.

5.6. Lemma. Let an integral matrix $M \in \mathcal{M}$ contain an $m$-antichain of size $n$ for some $m, n \in \mathbf{N}$, and let $M^{\prime}=e_{l}^{\leftarrow}(M)$ for some $l \in \mathbf{N}$. Then $M^{\prime}$ also contains an $m$-antichain of size $n$.

Proof. Let the application of $e_{j}^{\leftarrow}$ result in a transfer from $M_{k, l+1}$ to $M_{k, l}$. Consider the subset $S$ of antichains in the $m$-antichain the have a unit both in column $l$ and in column $l+1$, and put $r=\# S \geq 0$. Let $I, I^{\prime}$ be the $r$-multisets on $\mathbf{N}$ such that the units in column $l$ of the chains in $S$ form the multiset $\{(i, l) \mid i \in I\}$, and that the units in column $l+1$ of the chains in $S$ form the multiset $\left\{\left\{(i, l+1) \mid i \in I^{\prime}\right\}\right.$; write $I=\left\{\left\{i_{0}, \ldots, i_{r-1}\right\}\right\}$ and $I^{\prime}=\left\{\left\{i_{0}^{\prime}, \ldots, i_{r-1}^{\prime}\right\}\right\}$ with $i_{0} \leq \cdots \leq i_{r-1}$ and $i_{0}^{\prime} \leq \cdots \leq i_{r-1}^{\prime}$. Since each chain of $S$ contributes an index $i$ to $I$ and an index $i^{\prime}$ to $I^{\prime}$ with $i \leq i^{\prime}$, it follows that $i_{j} \leq i_{j}^{\prime}$ for all $j \in[r]$. We can then cut each chain in $S$ into a part in columns $\leq l$ and a part in columns $>l$, and recombine all the parts to form an $r$ antichain $\left(A^{(j)}\right)_{j \in[r]}$ with the same sum as $S$, in which the antichain $A^{(j)}$ has units both at $\left(i_{j}, l\right)$ and at $\left(i_{j}^{\prime}, l+1\right)$. We replace $S$ by this $r$-antichain, and set $A=\sum_{j \in[r]} A^{(j)}$.

If $A_{k, l+1}<M_{k, l+1}$ then either our $m$-antichain is already one in $M^{\prime}$ (if it does not use all units of $M$ at $(k, l+1)$ ), or it contains an antichain with a unit at $(k, l+1)$ and no units in column $l$, in which we can then replace its unit at $(k, l+1)$ by one at $(k, l)$; in both cases we have found an $m$-antichain of size $n$ in $M^{\prime}$. Now assume that $A_{k, l+1}=M_{k, l+1}$ and let $t=\max \left\{j \mid i_{j}^{\prime}=k\right\}$ be the index of the last contribution to $A_{k, l+1}$. Definition 1.4 .1b gives $\sum_{i=i_{j}}^{k-1}\left(M_{i+1, l+1}-M_{i, l}\right) \geq 1$ for all $j \leq t$, and the antichains $A^{(j)}, \ldots, A^{(t)}$ give null contributions to the left hand side; therefore either $M_{i, l+1}>A_{i, l+1}$ holds for some $i$ with $i_{j}<i<k$, or $j>0$ and $i_{j-1}^{\prime}>i_{j}$. Then we can set $k^{-}=\max \left\{i<k \mid A_{i, l+1}<M_{i, l+1}\right\}$ and $s=\min \left\{j \mid i_{j}^{\prime} \geq k^{-}\right\} \leq t$, and we have $i_{s}<k^{-}$and $i_{j}<i_{j-1}^{\prime}$ whenever $s<j \leq t$.

If our $m$-antichain does not use all $M_{k^{-}, l+1}$ available units at $\left(k^{-}, l+1\right)$, then one can add such a unit to the antichain $A^{(s)}$ to replace the one at $\left(i_{s}^{\prime}, l+1\right)$, and then replace for all $s<j \leq t$ the unit at $\left(i_{j}^{\prime}, l+1\right)$ in $A^{(j)}$ by one at $\left(i_{j-1}^{\prime}, l+1\right)$ (some such replacements may give back the same antichain); thus our $m$-antichain is replaced by another $m$-antichain of size $n$ in $M$ that does not use all units of $M$ at $(k, l+1)$, and is 


\section{Characterisation of the implicit shape}

therefore also an $m$-antichain in $M^{\prime}$. If our $m$-antichain does use all units at $\left(k^{-}, l+1\right)$, then it contains at least one antichain $A$ with such a unit and without any unit in column $l$. Any units of $A$ in columns $<l$ can be combined with the units of $A^{(t)}$ in columns $>l$ to from a new antichain $B$, while the units of $A$ in columns $>l$ can be combined with the units of $A^{(s)}$ in columns $\leq l$; furthermore for $s<j \leq t$ the units of $A^{(j)}$ in columns $\leq l$ can be combined with the units of $A^{(j-1)}$ in columns $>l$. Thus we have reorganised our $m$-antichain into another one of the same size in $M$, containing an antichain $B$ with a unit at $(k, l+1)$ that can be replaced by one at $(k, l)$.

We are now set to prove theorem 5.2; the proof is just a formalisation of the argument given before our sequence of lemmas.

Proof of theorem 5.2. If $M, M^{\prime}$ are a binary or integral $k \times l$ matrices, and $M^{\diamond}, M^{\prime \diamond}$ are obtained from them by a rotation by a half turn (mapping the origin to $(k-1, l-1)$ ), then the relation $M^{\prime}=e_{i}^{\uparrow}(M)$ with $i \in[k-1]$ is equivalent to $M^{\prime \diamond}=f_{k-2-i}^{\downarrow}\left(M^{\diamond}\right)$, while $M^{\prime}=e_{j}^{\leftarrow}(M)$ with $j \in[l-1]$ is equivalent to $M^{\prime \diamond}=f_{l-2-j}\left(M^{\diamond}\right)$. Moreover, the rotation maps chains to chains and antichains to antichains. Then for each of the four preceding lemmas, one can derive the corresponding statement for the crystal operation in the opposite direction by applying the lemma to $M^{\diamond}$. Therefore each lemma may be strengthened to " $M$ contains an $m$-(anti)chain of size $n$ if and only if $M^{\prime}$ contains one" (lemma 5.5 speaks of an $m$-chain of size at least $n$, but this is only to make its proof easier to read; such an $m$-chain exists if and only if an $m$-chain of size $n$ exists). Furthermore, applying rotation by a quarter turn to the two lemmas for binary matrices gives the corresponding statements with $m$-chains replaced by $m$ antichains, and with horizontal and vertical moves interchanged, while the two lemmas for integral matrices give the corresponding statements with horizontal and vertical moves interchanged by transposition. We may conclude both in the binary and the integral case that if $M$ and $M^{\prime}$ are related by any crystal operation, then $M$ contains an $m$-chain of size $n$ if and only if $M^{\prime}$ does so, and the same for $m$-antichains. The theorem now follows from the fact that it holds for normal forms, as we had already observed.

As the proofs above indicate, whenever $M$ and $M^{\prime}$ are related by a crystal operation, one can obtain a $m$-(anti)chain in $M^{\prime}$ of maximal size by choosing an appropriate such $m$-(anti)chain in $M$ and either leaving its sum unchanged or moving one unit between the same squares as in the application of the crystal operation. Nevertheless, due to the fact that the moving unit may migrate from one (anti)chain to another and to the necessity of switching from one $m$-(anti)chain to another with a different sum between successive crystal operations, the composition of the maximal $m$-(anti)chains in $M$ and the relations between them may be different from those in the normal form $\operatorname{Diag}^{[2]}(\lambda)$ or $\operatorname{Diag}(\lambda)$ where $\lambda=\pi(M)$. Notably the distribution of sizes of individual (anti)chains may be necessarily different: while in normal forms such $m$-chains always decompose into chains of sizes $\lambda_{0}, \ldots, \lambda_{m-1}$, an $m$-chain of maximal size for $m>1$ need in general not even contain any individual chain of maximal size $\lambda_{0}$. Here are examples of such a binary and integral matrix, both with $\lambda=(5,3,1)$, whose unique 2-chain of size 8 only 
decomposes into two chains of size 4 , and fail to contain the unique chain of size 5:

$$
\left(\begin{array}{lllll}
1 & 1 & 0 & 1 & 1 \\
0 & 0 & 1 & 0 & 0 \\
1 & 1 & 0 & 1 & 1
\end{array}\right) \in \mathcal{M}^{[2]} \text { and } \quad\left(\begin{array}{lllll}
2 & 0 & 0 & 2 & 0 \\
0 & 0 & 1 & 0 & 0 \\
0 & 2 & 0 & 0 & 2
\end{array}\right) \in \mathcal{M}
$$

(both examples were directly inspired by the one given in [Gre1, §3]). If one would trace the evolution of maximal $m$-chains from their normal form back to these matrices for $m=1$ and $m=2$, using the appropriate lemmas above, then it is clear that at some point the two cases must diverge; there is therefore no hope of improving those lemmas so that they keep track of complete decompositions into (anti)chains from which $\mathrm{m}$ (anti)chains of appropriate sizes could be extracted for all $m \in \mathbf{N}$. The only thing one can say in general about the sizes of individual chains in a $m$-chain of maximal size is that they lie in the range from $\lambda_{m-1}$ to $\lambda_{0}$ inclusive: they obviously cannot exceed the maximal size $\lambda_{0}$ of individual chains, and if any had size strictly less than $\lambda_{m-1}$, then could remove that chain and be left with an $(m-1)$-chain of size exceeding $\sum_{i \in[m-1]} \lambda_{i}$, which is impossible.

The property exhibited by the example allows another negative conclusion to be drawn: if $\mathcal{M}^{[2]}$ and $\mathcal{M}$ are considered as partially ordered sets by ' $\subseteq$ ' (componentwise comparison) and $\mathcal{P}$ is considered so by inclusion of diagrams, then the implicit shape does not define a poset morphism. After all, the unit in the middle of the matrix is contained in any 1-chain of maximal size but not in all 2-chains of maximal size (in fact there is only one such $m$-chain in either case), so that suppression of this unit will decrease the initial part of $\pi(M)$ without decreasing the sum of its first two parts; consequently the diagram of $\pi(M)=(5,3,1)$ does not contain that of $\pi\left(M^{\prime}\right)=(4,4)$ where $M^{\prime}$ is obtained from $M$ by replacing the middle entry by 0 .

To prove that a given $m$-chain has maximal size it is not necessary to find all $\mathrm{m}$ chains, but it suffices to produce any $\lambda_{m}$-antichain of maximal size. This is so because of the following general property, in which we define the "union" of two matrices as $C \cup A=C+A-C \cap A=\left(\max \left(C_{i, j}, A_{i, j}\right)\right)_{i, j \in \mathbf{N}}$ :

5.7. Proposition. Let $M$ be a binary or integral matrix, and $\lambda=\pi(M)$. If for some $k, l>0$ and some sum $C$ of a $k$-chain in $M$ and a sum $A$ of an l-antichain in $M$ one has $|C \cap A|=k l$ and $M=C \cup A$, then the $k$-chain and the l-antichain are both of maximal size, and $\lambda_{k} \leq l \leq \lambda_{k-1}$, in other words $(k-1, l-1) \in[\lambda]$ and $(k, l) \notin[\lambda]$. Conversely for any $k, l$ satisfying $\lambda_{k} \leq l \leq \lambda_{k-1}$, the sum $C$ of any $k$-chain of maximal size in $M$ and the sum $A$ of any l-antichain of maximal size in $M$ satisfy $|C \cap A|=k l$ and $M=C \cup A$.

Proof. Since for any sum $C^{\prime}$ of a $k$-chain in $M$ and a sum $A^{\prime}$ of an $l$-antichain in $M$ one has $\left|C^{\prime} \cap A^{\prime}\right| \leq k l$, and therefore $\left|C^{\prime} \cup A^{\prime}\right| \geq\left|C^{\prime}\right|+\left|A^{\prime}\right|-k l$, while on the other hand $M \supseteq C^{\prime} \cup A^{\prime}$ implies $\left|C^{\prime} \cup A^{\prime}\right| \leq|M|=|C|+|A|-k l$, it is clear that in the first part $|C|$ and $|A|$ are maximal among the sizes of $k$-chains and of $l$-antichains in $M$, respectively. Now let $C$ be the sum of any $k$-chain in $M$ of maximal size, so $|C|=\sum_{i \in[m]} \lambda_{i}$, and let $A$ be the sum of any $l$-antichain in $M$ of maximal size, so 
$|A|=\sum_{j \in[l]} \lambda_{j}^{t}$; put $d=k l-|C \cap A|$ and $d^{\prime}=|M-C \cup A|$ (so in the case just considered one has $\left.d=d^{\prime}=0\right)$. The expression giving $|C|$ can be interpreted as $\left.\#[\lambda]\right|_{[k] \times \mathbf{N}}$ (where $\left.[\lambda]\right|_{X}$ abbreviates $\left.[\lambda] \cap X\right)$, the number of squares in the topmost $k$ rows of $[\lambda]$. Similarly the expression for $|A|$ can be interpreted as $\left.\#[\lambda]\right|_{\mathbf{N} \times[l]}$. Therefore the equation $|C|+|A|=|C \cap A|+|C \cup A|$ can be written as

$$
\left.\#[\lambda]\right|_{[k] \times \mathbf{N}}+\left.\#[\lambda]\right|_{\mathbf{N} \times[l]}=\#([k] \times[l])-d+\#[\lambda]-d^{\prime} .
$$

Now the only squares in $\mathbf{N}^{2}$ with different contributions to the sets counted on the left and on the right are those in $D=[k] \times[l] \backslash[\lambda]$ or in $D^{\prime}=\left.[\lambda]\right|_{(\mathbf{N}-[k]) \times(\mathbf{N}-[l])}$, and in both cases they contribute 1 to the right hand side and 0 to the left hand side. Therefore $d+d^{\prime}=\# D+\# D^{\prime}$, and we may conclude that $d=d^{\prime}=0$ if and only if $D=D^{\prime}=\emptyset$, which is equivalent to $(k-1, l-1) \in[\lambda]$ and $(k, l) \notin[\lambda]$. The proposition follows.

Note that although in the case of normal forms one even has $d=\# D$ and $d^{\prime}=\# D^{\prime}$, this is not true in general: in the examples given the sum $C$ of the 2-chain of maximal size is also the sum of a 4 -antichain, so in this case with $(k, l)=(4,2)$ and $A=C$ one has $d=0$ but $D=\{(3,1)\}$.

While the proposition allows an easy certification of the maximality of a $k$-chain and an $l$-antichain if an appropriate pair is given, it does not provide a procedure for obtaining such a pair. The best algorithmic method we know of to determine such witnesses for (partial information about) the implicit shape of a matrix $M$ is to apply crystal operations to $M$ until reaching a form in which such witnesses are evident (for instance a matrix encoding a straight tableau), and then trace back one's steps using the descriptions in the proofs of the lemmas of this section to find corresponding witnesses in $M$. This is somewhat laborious to do by hand, but still feasible for the matrices of our running examples. Here it is easiest to reduce to the form that encodes the straight tableau $S$ of (31), namely is the matrix $Q$ of (28) in the binary case, which is reached after 12 leftward moves, or the matrix $P$ of (29) in the integral case, which is reached after 13 upward unit transfers. After this reduction we can find maximal $k$-chains and $l$-antichains for every $k$ and $l$ by taking in the binary case the $k$ topmost units (or all units if there are less than $k$ ) in every column of $Q$ respectively the units in the $l$ leftmost rows of $Q$, or in the integral case the $k$ topmost rows of $P$ respectively the $l$ leftmost units (or all available units) in every row of $P$ (in both cases these correspond to the $k$ topmost rows and the $l$ leftmost columns of the display of $S$ ). As an example, tracing that maximal 3-chain of size 13 and that maximal 4-antichain of size 16 back to $M$, we find in $M$ the 3 -chain coloured red, green and blue in the following displays

$$
\left(\begin{array}{lllllllll}
0 & 1 & 0 & 0 & 1 & 0 & 0 & 0 & 0 \\
1 & 1 & 1 & 0 & 0 & 0 & 0 & 0 & 0 \\
1 & 0 & 1 & 0 & 0 & 1 & 0 & 0 & 0 \\
0 & 1 & 0 & 1 & 0 & 0 & 0 & 0 & 0 \\
0 & 0 & 1 & 1 & 1 & 0 & 1 & 0 & 0 \\
1 & 0 & 0 & 0 & 1 & 0 & 0 & 1 & 1 \\
0 & 1 & 1 & 1 & 1 & 1 & 1 & 1 & 0
\end{array}\right) \quad\left(\begin{array}{lllllll}
1 & 0 & 1 & 0 & 1 & 2 & 0 \\
1 & 1 & 0 & 1 & 1 & 0 & 3 \\
0 & 2 & 1 & 0 & 1 & 1 & 0 \\
0 & 0 & 1 & 1 & 1 & 0 & 2 \\
0 & 0 & 0 & 0 & 0 & 1 & 2
\end{array}\right)
$$


while the two 4-antichains one obtains in both cases can be described as follows by the sets of positions where their individual antichains have units: in the binary case these are $\{(0,1),(1,0),(2,0),(5,0)\},\{(0,3),(1,1),(3,1),(6,1)\},\{(1,2),(2,2)$, $(4,2),(6,2)\}$ and $\{(2,5),(3,3),(4,3),(6,3)\}$, and in the integral case these sets are $\{(1,0),(2,1),(3,3),(4,6)\},\{(0,0),(2,1),(3,5),(4,6)\},\{(1,1),(2,2),(3,3),(4,5)\}$ and $\{(0,2),(1,3),(2,4),(3,6)\}$. The most complicated of these computations is for the 4 antichain in the integral case, where cases that require recombination of chains occur frequently; this explains the fact that the result for this case are rather tangled. There is also some liberty in interpreting the transitions prescribed in the proofs of the lemmas, since more than one antichain can contain a given unit; one could also perform some intermediate untangling of antichains, which would affect the final result as well.

It is interesting to compare the results above with the original approach of [Gre1]. There the roles of chains and antichains are played by increasing and decreasing subsequences extracted from a given finite sequence of numbers. In fact only sequences representing permutations are considered, but that restriction can be lifted if for chains one allows weakly increasing sequences while for antichains one requires strictly decreasing sequences; everything generalises immediately by treating equal entries as if they were increasing from left to right (a price to pay is that the theory becomes asymmetric, and the opposite convention would have been equally feasible, but we shall stick to this more usual convention). One can associate words to matrices in a way that is compatible with this interpretation of chains and antichains: for a binary matrix one takes concatenation of the strictly decreasing words whose weights are given by the columns of the matrix taken from left to right, while for an integral matrix one takes the concatenation of the weakly increasing words whose weights are given by the rows of the matrix from bottom to top. While this correspondence seems biased towards words that have this particular composition, one can in fact model words bijectively by matrices, by restricting the latter to those whose column sums (in the binary case) or row sums (in the integral case) are all 1 up to some point beyond which they are all 0: the mentioned concatenations then combine words consisting of a single letter.

The invariance of the maximal size of $m$-chains or of $m$-antichains is then shown for a certain type of transposition of letters in the word, which generates an equivalence relation on words that is known by [Knu, Theorem 6] to characterise words with the same insertion tableau in the RSK correspondence. To be precise, the interchange of a pair of distinct adjacent letters is permitted in the presence of a letter $l$ directly to the left or to the right of the pair, whose value is either strictly in between those of the letters being interchanged, or (in keeping with the interpretation of equal letters) if either $l$ is to the left of the pair and its value equal to the larger of the letters being interchanged, or $l$ is to the right of the pair and its value equal to the smaller one. If $a, b, c$ designate letters with $a<b<c$, these transpositions can therefore be represented as transformations $b a c \leftrightarrow b c a, a c b \leftrightarrow c a b, b a b \leftrightarrow b b a$ or $a b a \leftrightarrow b a a$ of three consecutive letters; we shall call them elementary Knuth transformations.

Modelling words by matrices, the fact that elementary Knuth transformations leave unchanged the RSK insertion tableau for the word is reflected by the fact that they can be realised by horizontal moves within the three columns (in the binary case) or vertical 
transfers between the three rows (in the integral case) corresponding to the letters concerned. We illustrate this in the binary case for transition $b a c \rightarrow b c a$ :

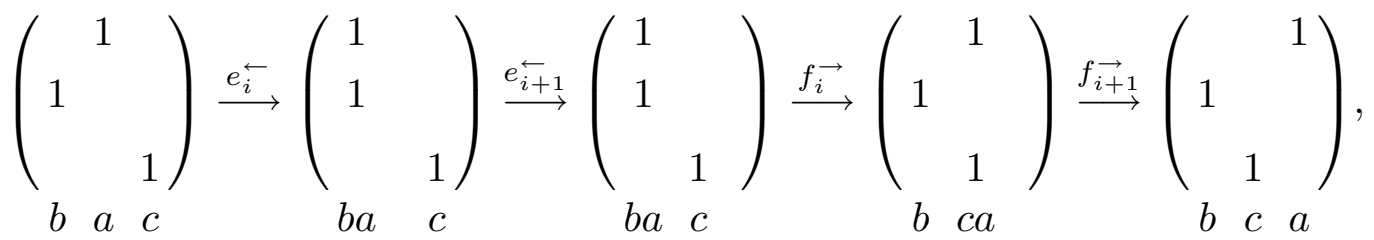

and similarly for a transition $a b a \rightarrow b a a:$

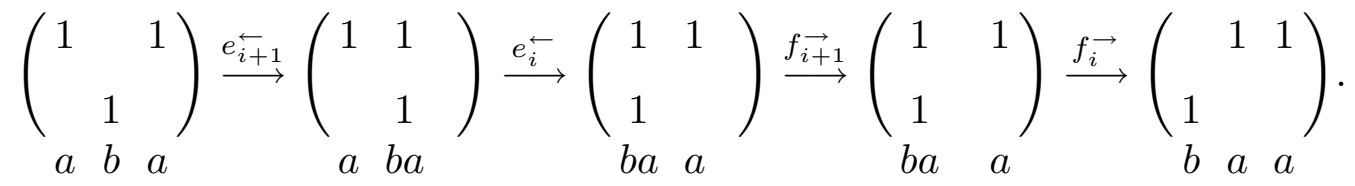

The word corresponding to the matrix changes only during the application of $f_{i}$ in the first case, and that of $e_{i}^{\leftarrow}$ in the second. The integral case is similar; we show just these essential transitions for $b a c \rightarrow b c a$ and for $b a b \rightarrow b b a$ (keeping in mind that we must read from bottom to top):

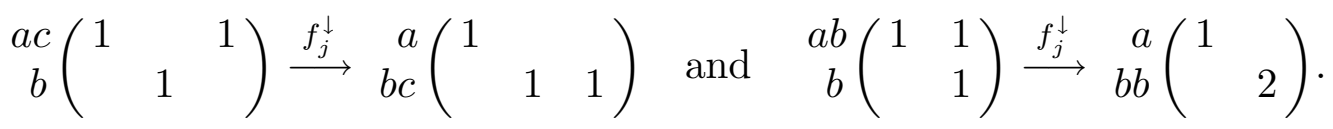

It is clear that if these transformations are made inside a larger matrix $M$, then the one of the matrices $P, Q$ associated to $M$ in theorem 3.1.3 that is obtained by exhausting crystal operations in a direction parallel to those used here does not change; this matrix is $Q$ in the binary case and $P$ in the integral case. If $M$ encodes a skew tableau $T$ with at most one square in any row or column, then that matrix encodes both in the binary and the integral case the rectification $S$ of $T$, which is also the RSK insertion tableau for the word read off from the display of $T$ from bottom left to top right.

It is less obvious that by repeating elementary Knuth transformations, one can transform the word corresponding to $M$ into the word corresponding to the matrix that encodes $S$, which word can be read off from the display of $S$ in the reverse Kanji (binary case) of reverse Semitic (integral case) order. The proof of [Knu, Theorem 6] is based on the fact that Schensted insertion can be emulated using elementary Knuth transformations: if $T^{\prime}=T \leftarrow x$ is the result of row-inserting an entry $x$ into a semistandard Young tableau $T$, and $t$ and $t^{\prime}$ are the words obtained by reading the displays of $T$ and $T^{\prime}$ in reverse Semitic order, then $t^{\prime}$ can be obtained from $t x$ by a sequence of elementary Knuth transformations (see also [Fult, §2.1]; the transpositions proceed in a single sweep from right to left). A similar result can easily be shown for column insertion $x \rightarrow T$, reading the tableaux in reverse Kanji order.

Since we have seen that elementary Knuth transformations can be realised by crystal operations, one may ask if one can conversely derive, from the exhaustion of crystal operations that transforms $M$ into the matrix encoding $S$, a sequence of elementary Knuth transformations that realises the transformation of the corresponding words. Of course, since we remain inside one Knuth equivalence class, successive words obtained 
during the exhaustion of the crystal operations can be linked by elementary Knuth transformations, but the number of them required at each step turns out to be surprisingly large. It is shown in [Schü2, 2.3.bis] how the change to the reverse Semitic reading of a tableau (with a hole) caused by the upward move of one entry occurring during an inward jeu de taquin slide can be realised by a sequence of elementary Knuth transformations (the goal of that statement was in fact to show that only the directions $b c a \rightarrow b a c, b b a \rightarrow b a b, a c b \rightarrow c a b$, or $a b a \rightarrow b a a$ are needed here; it should be noted that some transpositions might also allow an interpretation as a transformation in the opposite sense within another triplet). That case corresponds precisely to an upward unit transfer in an integral matrix. It is not quite clear how many transpositions are produced in the proof of the cited result, although it seems to be larger than necessary. We shall sharpen that result by providing a count of the transpositions used; experimental evidence strongly suggests that the number we give is in fact minimal.

5.8. Proposition. Let $M \in \mathcal{M}$ allow an upward unit transfer from $\left(i_{0}+1, j_{0}\right)$ to $\left(i_{0}, j_{0}\right)$ resulting in $M^{\prime}=e_{i_{0}}^{\uparrow}(M)$, and let the words $w, w^{\prime}$ be the concatenations of the weakly increasing words with weights given by the rows of $M$ and of $M^{\prime}$, respectively, ordered from bottom to top. Then $w$ can be transformed into $w^{\prime}$ by a succession of $n^{2}$ elementary Knuth transformations of type $b c a \rightarrow b a c, b b a \rightarrow b a b, a c b \rightarrow c a b$, or $a b a \rightarrow b a a$, where $n=\sum_{j<j_{0}} M_{i_{0}, j}+\sum_{j>j_{0}} M_{i_{0}+1, j}$.

Proof. For brevity we take the designation $b c a \rightarrow b a c$ to include type $b b a \rightarrow b a b$ as well, and $a c b \rightarrow c a b$ is similarly taken to include type $a b a \rightarrow b a a$. Put $k=\sum_{j<j_{0}} M_{i_{0}, j}$ and $l=\sum_{j>j_{0}} M_{i_{0}+1, j}$, so that $k+l=n$. Write the rightmost $n+1$ letters of the subword corresponding to row $i_{0}+1$ of $M$ as $c_{1}, \ldots, c_{k}, j_{0}, d_{1}, \ldots, d_{l}$ and the leftmost $n$ letters of the subword corresponding to row $i_{0}$ of $M$ as $a_{1}, \ldots, a_{k}, b_{1}, \ldots, b_{l}$. The inequalities of definition 1.4.1a guarantee that the letters $c_{1}, \ldots, c_{k}$ and $b_{1}, \ldots, b_{l}$ exist in those rows; the concatenation of these weekly increasing words forms a subword $v=$ $c_{1} \cdots c_{k} j_{0} d_{1} \cdots d_{l} a_{1} \cdots a_{k} b_{1} \cdots b_{l}$ of $w$ in which all transpositions will take place, and we shall ignore all other letters. The same inequalities also imply that $a_{i}<c_{i}$ for $1 \leq i \leq k$ and $b_{i}<d_{i}$ for $1 \leq i \leq l$, and if $l>0$ one has $j_{0} \leq b_{1}$; thus $v$ corresponds to the reverse Semitic reading of a tableau at an intermediate stage of a jeu de taquin slide, when moving $j_{0}$ upward in the situation

\begin{tabular}{|l|l|l|l|l|l|l|l|l|}
\hline$a_{1}$ & $\cdot$ & $\cdot$ & $a_{k}$ & & $b_{1}$ & $\cdot$ & $\cdot$ & $b_{k}$ \\
\hline$c_{1}$ & $\cdot$ & $\cdot$ & $c_{k}$ & $j_{0}$ & $d_{1}$ & $\cdot$ & $\cdot$ & $d_{k}$ \\
\hline
\end{tabular}

We must transformation $v$ into $v^{\prime}=c_{1} \cdots c_{k} d_{1} \cdots d_{l} a_{1} \cdots a_{k} j_{0} b_{1} \cdots b_{l}$, which we shall do explicitly by the following choreography. We proceed in $n$ sweeps of $n$ elementary Knuth transformations each, starting the successive sweeps with the triplets whose rightmost letters are successively $a_{1}, \ldots, a_{k}, b_{1}, \ldots, b_{l}$, and proceeding from right to left within each sweep. During sweep $i$ with $1 \leq i \leq k$, the letter $a_{i}$ is first shifted to the left $n-i+1$ places by transformations of type $b c a \rightarrow b a c$, after which it ends up to the right of the letter $c_{i}$; that letter is then shifted to the left $i-1$ places across the letters $a_{1}, \ldots, a_{i-1}$ by transformations of type $a c b \rightarrow c a b$, which results 


\section{Characterisation of the implicit shape}

in a word $c_{1} \cdots c_{i} a_{1} \cdots a_{i} c_{i+1} \cdots c_{k} j_{0} d_{1} \cdots d_{l} a_{i+1} \cdots a_{k} b_{1} \cdots b_{l}$. After these $k$ sweeps one therefore has the word $c_{1} \cdots c_{k} a_{1} \cdots a_{k} j_{0} d_{1} \cdots d_{l} b_{1} \cdots b_{l}$. During sweep $k+i$ with $1 \leq i \leq l$, the letter $b_{i}$ is first shifted to the left $l-i$ places across the letters $d_{i+1}, \ldots, d_{l}$ by transformations of type $b c a \rightarrow b a c$ in which it has the role of $a$, after which it finds itself to the right of the letter $d_{i}$; that letter is then shifted to the left $k+i$ places by transformations of type $a c b \rightarrow c a b$, which results in a word $c_{1} \cdots c_{k} d_{1} \cdots d_{i} a_{1} \cdots a_{k} j_{0} b_{1} \cdots b_{i} d_{i+1} \cdots d_{l} b_{i+1} \cdots b_{l}$. After these sweeps we have the word $v^{\prime}$.

For a leftward move in a binary matrix from $\left(i_{0}, j_{0}+1\right)$ to $\left(i_{0}, j_{0}\right)$, there is a similar realisation of the change in the corresponding words, which is the change in the reverse Kanji reading of a tableau that occurs during a leftward step in a jeu de taquin slide, using $n^{2}$ elementary Knuth transformations of the same types, where $n=\sum_{i<i_{0}} M_{i, j_{0}}+\sum_{i>i_{0}} M_{i, j_{0}+1}$. The proof is the same, interchanging left and right in the word, and with the implied interchange of weak and strict inequalities.

The proposition indicates that the number of elementary Knuth transformations corresponding to the exhaustion of upward crystal operations can be considerably larger than the number of such operations; in our example we needed 13 upward unit transfers, which correspond to 150 elementary Knuth transformations. This can be improved a bit when there are successive transfers between the same pair of rows: if there are $k$ such transfers then all involve the same value of $n$, and it appears that by an adaptation of the transposition procedure one can achieve the combined transformation of the word in $n(n+k-1)$ elementary Knuth transformations instead of $k n^{2}$; in the example this allows the total number of steps to be reduced to 122 . This is still worse than the number needed for the traditional emulation of the RSK insertion, although it is of the same order of magnitude: a straightforward application of that procedure to the word corresponding to $M$ gives a sequence of 120 transpositions, which can be reduced to 88 by observing that the first 32 transpositions bring back the word to its original form, due to the circumstance that the word corresponding to the three bottom rows of $M$ is already the reverse Semitic reading of a straight tableau. It should be noted that our matrix $M$ was chosen as the integral encoding of a skew semistandard tableau that is fairly close to its rectification, whence the numbers above are relatively small. These data suggest that for the purpose of finding maximal $m$-(anti)chains in a binary or integral matrix, there is some advantage in using the transformations described in our lemmas rather than the (somewhat simpler) ones corresponding to elementary Knuth transformations.

Another approach to finding maximal $m$-(anti)chains would be to try to augment the inductive computation of $\pi(M)$ via growth diagrams with additional data, keeping track of maximal $m$-(anti)chains as witnesses for the implicit shape of submatrices. It appears however that this can only be done for $m=1$. 


\section{$\S$ 6. Remaining proofs.}

We shall finally provide, for completeness, proofs of statements for which they were so far omitted. The first such statement was the fact that crystal operations can be defined in such a way for any semistandard tableau $T$ that they correspond both to vertical crystal operations on its binary encoding $M$, and to horizontal crystal operations on it integral encoding $N$.

Proof of proposition 2.1. We know that if either $e_{m}^{\uparrow}(M)$ or $e_{m}^{\leftarrow}(N)$ is defined, then like $M$ or $N$ it satisfies the tableau condition for $\lambda / \kappa$ (propositions 1.3 .6 and 1.4.6), and is therefore the binary or integral encoding of some tableau $T^{\prime}$. Moreover $T^{\prime}$ can easily be seen to differ from $T$ only in the partition $\lambda^{(m+1)}$, for which some square $(k, l)$ has been added to its diagram to form the diagram of the corresponding partition in $T^{\prime}$ (in terms of the display of $T$, an entry $m+1$ in that square has been changed to $m$ ). We have to show that if the other type of encoding is taken of $T$ and of $T^{\prime}$, then the matrices obtained are also related by the appropriate raising operation. By interchanging the roles of $T$ and of $T^{\prime}$, this will also prove the statement about $f_{m}^{\downarrow}$ and $f_{m}$.

Suppose first that the binary encodings of $T$ and $T^{\prime}$ are $M$ and $e_{m}^{\uparrow}(M)$, and let their integral encodings be $N, N^{\prime}$, respectively. Then with $k, l$ as above, we know that the bits $M_{m, l}=0$ and $M_{m+1, l}=1$ are interchangeable in $M$, and we must show that a unit transfer is possible from $N_{k, m+1}$ to $N_{k, m}$ in $N$. Consider the left member $\sum_{i=k}^{k^{\prime}-1}\left(N_{i, m}-N_{i+1, m+1}\right)$ of the second inequality in definition 1.4.1b, which we must show to be nonnegative for any $k^{\prime}>k$. It counts the number of squares of the skew diagram $\left[\lambda^{(m+1)} / \lambda^{(m)}\right]$ that lie in rows $i \in\left[k^{\prime}\right]-[k]$, minus the number of squares of $\left[\lambda^{(m+2)} / \lambda^{(m+1)}\right]$ in rows $i+1 \in\left[k^{\prime}+1\right]-[k+1]$. Now these squares can also be described as those that lie in columns $j \in[l]-\left[l^{\prime}\right]$ where $l^{\prime}=\lambda_{k^{\prime}}^{(m+1)} \leq l=\lambda_{k}^{(m+1)}$, since squares of $\left[\lambda^{(m+1)} / \lambda^{(m)}\right]$ in row $k$ are in columns $<l$ while squares of $\left[\lambda^{(m+2)} / \lambda^{(m+1)}\right]$ in row $k$ are in columns $\geq l$, and similarly for $k^{\prime}$ and $l^{\prime}$. It follows that $\sum_{i \in\left[k^{\prime}\right]-[k]}\left(N_{i, m}-N_{i+1, m+1}\right)=$ $\sum_{j \in[l]-\left[l^{\prime}\right]}\left(M_{m, j}-M_{m+1, j}\right)$ which is nonnegative by hypothesis. For the first inequality in definition 1.4.1b the argument is similar. Here it is easiest to take the version of that inequality for $N^{\prime}$, which is equivalent to the one for $N$ : for any $k^{\prime}<k$ one puts $l^{\prime}=\lambda_{k^{\prime}}^{(m+1)}$ (which satisfies $\left.l^{\prime} \geq l+1\right)$, and one shows that $\sum_{i \in[k]-\left[k^{\prime}\right]}\left(N_{i+1, m+1}^{\prime}-\right.$ $\left.N_{i, m}^{\prime}\right)=\sum_{j \in\left[l^{\prime}\right]-[l+1]}\left(M_{m+1, j}-M_{m, j}\right)$, which is nonnegative. This concludes the proof that $N^{\prime}=e_{m}^{\leftarrow}(N)$.

Now suppose that the integral encodings of $T$ and $T^{\prime}$ are $N$ and $e_{m}^{\leftarrow}(N)$, and let their binary encodings be $M, M^{\prime}$, respectively. The argument we have just given shows that, among the inequalities in definition 1.3.1a that must be shown to hold in order to justify $M^{\prime}=e_{m}^{\uparrow}(M)$, those for which $l^{\prime} \in\left\{\lambda_{k^{\prime}}^{(m+1)} \mid k^{\prime}>k\right\} \cup\{l\} \cup\left\{\lambda_{k^{\prime}}^{(m+1)}-1 \mid k^{\prime}<k\right\}$ are satisfied. This does not cover all inequalities, but the values in rows $m, m+1$ of $M$ that are incorporated into the summations on $j$ between such values of $l^{\prime}$ have a simple structure, as they account for squares of $\left[\lambda^{(m+2)} / \lambda^{(m+1)}\right]$ in row $k^{\prime}$, and squares of $\left[\lambda^{(m+1)} / \lambda^{(m)}\right]$ in row $k^{\prime}-1$, for some fixed $k^{\prime}$ depending on the interval considered. In such an interval one has from left to right: a possibly empty sequence of columns $\left(\begin{array}{l}0 \\ 1\end{array}\right)$, a possibly empty sequence either of columns $\left(\begin{array}{l}0 \\ 0\end{array}\right)$ or $\left(\begin{array}{l}1 \\ 1\end{array}\right)$, and a possibly 


\section{Remaining proofs}

empty sequence of columns $\left(\begin{array}{l}1 \\ 0\end{array}\right)$; similarly the columns incorporated into the partial sums after the largest such value of $l^{\prime}$ can only be $\left(\begin{array}{l}0 \\ 1\end{array}\right)$ or $\left(\begin{array}{l}0 \\ 0\end{array}\right)$. It follows easily from this description that the required inequalities also hold at the remaining values of $l^{\prime}$.

The next statement left to prove is that the involutions defined by crystal operations on matrices satisfy the Coxeter relations of type $A$. We did not use this fact anywhere, but it does represent an interesting aspect of crystal operations, so we present the proof, even if this turns out to require quite a bit of work.

Proof of lemma 2.3. This is a statement about the structure of crystals, so by proposition 2.1 it suffices to consider the involutions $\sigma_{l}^{\leftrightarrow}$ in the case of integral matrices. We have already seen that the $\sigma_{l}^{\leftrightarrow}$ are involutions (the first Coxeter relation), and the fact that $\sigma_{j}^{\leftrightarrow}$ and $\sigma_{l}^{\leftrightarrow}$ commute when $|l-j| \geq 2$ (the second Coxeter relation) follows from the fact that they operate on disjoint pairs of rows. Hence the only non-obvious point is the third Coxeter relation, but here a direct proof would be difficult, because for instance the effect of applying $e_{k}^{\uparrow}$ to $M$ on the operation of $e_{k+1}^{\uparrow}$ depends on $M$ in a complicated way. Instead we shall proceed by reducing the general case to one where a detailed analysis is feasible. In general, if $M^{\prime}$ is obtained from $M$ by applying an invertible operation that commutes with $\sigma_{l}^{\leftrightarrow}$ and with $\sigma_{l+1}^{\leftrightarrow}$, then the equation $\sigma_{l}^{\leftrightarrow} \sigma_{l+1}^{\leftrightarrow} \sigma_{l}^{\leftrightarrow}(M)=\sigma_{l+1}^{\leftrightarrow} \sigma_{l}^{\leftrightarrow} \sigma_{l+1}^{\leftrightarrow}(M)$ holds if and only if $\sigma_{l}^{\leftrightarrow} \sigma_{l+1}^{\leftrightarrow} \sigma_{l}^{\leftrightarrow}\left(M^{\prime}\right)=\sigma_{l+1}^{\leftrightarrow} \sigma_{l}^{\leftrightarrow} \sigma_{l+1}^{\leftrightarrow}\left(M^{\prime}\right)$ does; we shall use such operations to reduce $M$ to a simpler form.

First of all we replace $M$ by $M_{\mathbf{N} \times([l+3]-[l])}$ (the inverse operation of adding back the columns outside the range $\{l, l+1, l+2\}$ commutes with $\sigma_{l}^{\leftrightarrow}$ and $\sigma_{l+1}^{\leftrightarrow}$, which only look at the columns in that range), and we may then as well assume that $k=0$. Secondly we may assume that upward crystal operations applied to $M$, which commute with $\sigma_{l}^{\leftrightarrow}$ and $\sigma_{l+1}^{\leftrightarrow}$, are exhausted. Thus we have reduced $M$ to the form

$$
M=\left(\begin{array}{lll}
a & b & c \\
0 & d & e \\
0 & 0 & f
\end{array}\right) \in \mathcal{M} \quad \text { with } a \geq d \geq f \text { and } a+b \geq d+e .
$$

Although the proposition can be directly proved for this situation, as we shall indicate below, one can take a shortcut, as in the proof in [Loth], by further reducing the situation using the fact that the operations $\sigma_{j}^{\leftrightarrow}$ commute with cyclic permutations of the nonzero rows of the matrix, as stated in lemma 6.1 below. Doing so to the matrix $M$ of (64), moving row 0 below the two following ones, one can again exhaust upwards crystal operations. In fact using $e_{1}^{\uparrow}$ all $a+b+c$ units that were cycled to row 2 can be transferred up to row 1 , and then $e_{0}^{\uparrow}$ can be applied $a+b+c-\min (d, c+f)$ times to obtain a matrix

$$
M^{\prime}=\left(\begin{array}{ccc}
a & b+d & c^{\prime} \\
0 & 0 & e^{\prime} \\
0 & 0 & 0
\end{array}\right) \quad \text { where } e^{\prime}=\min (d, c+f) \text { and } c^{\prime}=c+f-e^{\prime}+e
$$

in which upwards transfers are once again exhausted. Thus the case of matrices of the form (64) has been reduced to the case of matrices of the form (65). The latter case is 
contained in the former, and we can repeat the steps the we applied to further reduce to the case of a one-line matrix $M^{\prime \prime}=\left(\begin{array}{ll}a & b+d \\ c & +e+f\end{array}\right)$. Now for such matrices one has $\operatorname{col}\left(M^{\prime \prime}\right)=M_{0}^{\prime \prime}$, and since we know that the effect of $\sigma_{i}^{\leftrightarrow}$ on $\operatorname{col}(M)$ is to transpose the parts $i$ and $i+1$, it is clear that $\sigma_{0}^{\leftrightarrow} \sigma_{1}^{\leftrightarrow} \sigma_{0}^{\leftrightarrow}\left(M^{\prime \prime}\right)=\sigma_{1}^{\leftrightarrow} \sigma_{0}^{\leftrightarrow} \sigma_{1}^{\leftrightarrow}\left(M^{\prime \prime}\right)=(c+e+f \quad b+d a)$.

We shall now state formally the commutation of operations $\sigma_{j}^{\leftrightarrow}$ with cyclic permutations of rows that we used. It is valid for binary matrices as well as for integral ones, and it also has an analogue for $\sigma_{i}^{\ddagger}$; since the statement is of independent interest we state it in its most general form.

6.1. Lemma. Let $n, m \in \mathbf{N}, d \in[n]$ and $d^{\prime} \in[m]$. Denote by $R$ either the set of matrices $M \in \mathcal{M}^{[2]}$ or of matrices $M \in \mathcal{M}$, such that $M=M_{[n] \times[m]}$, and by $\pi_{\mathrm{r}}$ and $\pi_{\mathrm{c}}$ operations on $R$ defined by the cyclic permutation of the rows $\left\{M_{i} \mid i \in[n]\right\}$ by $d$ places (so $\pi_{\mathrm{r}}(M)_{i, j}=M_{i+d \bmod n, j}$ for all $M \in R,(i, j) \in[n] \times[m]$ ), respectively by the cyclic permutation of the columns $\left\{M_{j}^{t} \mid j \in[m]\right\}$ by $d^{\prime}$ places $\left(\operatorname{so} \pi_{\mathrm{c}}(M)_{i, j}=M_{i, j+d^{\prime} \bmod m}\right)$. Then for all $k, l \in \mathbf{N}$ and $M \in R$ one has $\sigma_{l}^{\leftrightarrow}\left(\pi_{\mathrm{r}}(M)\right)=\pi_{\mathrm{r}}\left(\sigma_{l}^{\leftrightarrow}(M)\right)$ and $\sigma_{k}^{\downarrow}\left(\pi_{\mathrm{c}}(M)\right)=$ $\pi_{\mathrm{c}}\left(\sigma_{k}^{\uparrow}(M)\right)$.

Proof. By symmetry it will suffice to consider the statement concerning $\sigma_{l}^{\leftrightarrow}$. Clearly only the columns $l, l+1$ that are affected by $\sigma_{l}^{\leftrightarrow}$ are of any concern, so we may assume they are the only ones: $l=0$ and $m=2$. Note that the effect of $\pi_{\mathrm{r}}$ and $\sigma_{0}^{\leftrightarrow}$ on row and column sums is determined without knowing more details of $M$, and these effects commute, so our only worry is that both sides of the equation might give different matrices with the same row and column sums. We shall start considering the special case of integral $2 \times 2$ matrices (so $n=2$ as well) with $d=1$, so that $\pi_{\mathrm{r}}$ is the interchange of the two rows. We put $\operatorname{row}(M)=(s, t)=\operatorname{row}\left(\sigma_{0}^{\leftrightarrow}(M)\right)$, then $M$ has the form $M=\left(\begin{array}{ll}a & s-a \\ b & t-b\end{array}\right)$. One has $\delta=\operatorname{col}(M)_{1}-\operatorname{col}(M)_{0}=s+t-2(a+b)$ in (25), and the same value applies to $\pi_{\mathrm{r}}(M)$. By symmetry between $M$ and $\pi_{\mathrm{r}}(M)$ we may assume that $s \leq t$; we distinguish the cases with $s \leq a+b \leq t$ from those where either $a+b<s$ or $t<a+b$. The condition $s \leq a+b \leq t$ means on one hand that $\min (t-b, t-b-\delta) \geq a$, so that in this case the transfer for computing $\sigma_{0}^{\leftrightarrow}(M)$ takes place entirely in the bottom row, and on the other hand that $s-a \leq \min (b, b-\delta)$ so that the transfer for computing $\sigma_{0}^{\leftrightarrow}\left(\pi_{\mathrm{r}}(M)\right)$ takes place entirely in the top row of $\pi_{\mathrm{r}}(M)=\left(\begin{array}{cc}b & t-b \\ a & s-a\end{array}\right)$; as a consequence one has $\sigma_{0}^{\leftrightarrow}\left(\pi_{\mathrm{r}}(M)\right)=\left(\begin{array}{cc}b-\delta & t-b+\delta \\ a & s-a\end{array}\right)=\pi_{\mathrm{r}}\left(\sigma_{0}^{\leftrightarrow}(M)\right)$. If instead one has $a+b<s \leq t$, then it follows that $\delta>0$ and in fact $t-b>a>t-b-\delta$, whence we may write $\sigma_{0}^{\leftrightarrow}(M)=\left(e_{0}^{\leftarrow}\right)^{\delta}(M)=\left(e_{0}^{\leftarrow}\right)^{s-(a+b)}\left(\left(e_{0}^{\leftarrow}\right)^{t-(a+b)}(M)\right)$, in which expression $\left(e_{0}^{\leftarrow}\right)^{t-(a+b)}$ operates in the bottom row, after which the factor $\left(e_{0}^{\leftarrow}\right)^{s-(a+b)}$ operates in the top row. The computation of $\sigma_{0}^{\leftrightarrow}\left(\pi_{\mathrm{r}}(M)\right)$ and can be performed similarly using $s-a>b>$ $s-a-\delta$; the two computations can be summarised as follows:

$$
\begin{aligned}
M & =\left(\begin{array}{ll}
a & s-a \\
b & t-b
\end{array}\right) \stackrel{\left(e_{0}^{\leftarrow}\right)^{t-(a+b)}}{\longrightarrow}\left(\begin{array}{cc}
a & s-a \\
t-a & a
\end{array}\right) \stackrel{\left(e_{0}^{\leftarrow}\right)^{s-(a+b)}}{\longrightarrow}\left(\begin{array}{cc}
s-b & b \\
t-a & a
\end{array}\right)=\sigma_{0}^{\leftrightarrow}(M), \\
\pi_{\mathrm{r}}(M) & =\left(\begin{array}{ll}
b & t-b \\
a & s-a
\end{array}\right) \stackrel{\left(e_{0}^{\leftarrow}\right)^{s-(a+b)}}{\longrightarrow}\left(\begin{array}{cc}
b & t-b \\
s-b & b
\end{array}\right) \stackrel{\left(e_{0}^{\leftarrow}\right)^{t-(a+b)}}{\longrightarrow}\left(\begin{array}{cc}
t-a & a \\
s-b & b
\end{array}\right)=\sigma_{0}^{\leftrightarrow}\left(\pi_{\mathrm{r}}(M)\right) .
\end{aligned}
$$




\section{Remaining proofs}

Clearly $\sigma_{0}^{\leftrightarrow}\left(\pi_{\mathrm{r}}(M)\right)=\pi_{\mathrm{r}}\left(\sigma_{0}^{\leftrightarrow}(M)\right)$ in this case. In the remaining case $t<a+b$ one has, with $a^{\prime}=t-b$ and $b^{\prime}=s-a$, that $a^{\prime}+b^{\prime}<s$ and $M=\left(\begin{array}{cc}s-b^{\prime} & b^{\prime} \\ t-a^{\prime} & a^{\prime}\end{array}\right)$, so in fact $M=\sigma_{0}^{\leftrightarrow}\left(M^{\prime}\right)$ for a matrix $M^{\prime}=\left(\begin{array}{cc}a^{\prime} & s-a^{\prime} \\ b^{\prime} & t-b^{\prime}\end{array}\right)$ to which the previous case applies, which settles this final case.

Now we return to the more general case of $\sigma_{0}^{\leftrightarrow}$ operating on a $n \times 2$ matrix $M$ (either binary or integral). Write $M=\left(\begin{array}{c}S \\ T\end{array}\right)$ where $S$ is $d \times 2$ matrix and $T$ a $(n-d) \times 2$ matrix, so that $\pi_{\mathrm{r}}(M)=\left(\begin{array}{l}T \\ S\end{array}\right)$. Any horizontal crystal operation in $M$ occurring inside $S$ or $T$ will also be valid in that part considered in isolation. But definition 1.3.1b or 1.4.1b implies that if conversely $S \rightarrow S^{\prime}$ is a horizontal crystal operation that is valid in isolation, then $\left(\begin{array}{c}S \\ T\end{array}\right) \rightarrow\left(\begin{array}{c}S^{\prime} \\ T\end{array}\right)$ is valid in $M$ only if $\min \left(n_{0}(S), n_{0}\left(S^{\prime}\right)\right) \geq n_{0}^{\leftarrow}(T)$, while such an operation $T \rightarrow T^{\prime}$ gives rise to one $\left(\begin{array}{c}S \\ T\end{array}\right) \rightarrow\left(\begin{array}{c}S \\ T^{\prime}\end{array}\right)$ only if $n_{0}(S) \leq \min \left(n_{0}^{\leftarrow}(T), n_{0}^{\leftarrow}\left(T^{\prime}\right)\right)$. This means that the moves or transfers involved in the transition $M \rightarrow \sigma_{0}^{\leftrightarrow}(M)$ will be distributed between the parts $S$ and $T$ in the same way as for $\bar{M}=\left(\begin{array}{cc}a & s-a \\ b & t-b\end{array}\right) \in \mathcal{M}$ the transfers involved in the transition $\bar{M} \rightarrow \sigma_{0}^{\leftrightarrow}(\bar{M})$ are distributed between the two rows, where $a=n_{0}(S), \quad s=a+n_{0}^{\leftarrow}(S), \quad b=n_{0}(T)$, and $t=b+n_{0}^{\leftarrow}(T)$. So if $\sigma_{0}^{\leftrightarrow}(\bar{M})=\left(\begin{array}{cc}a+\delta_{0} & s-a-\delta_{0} \\ b+\delta_{1} & t-b-\delta_{1}\end{array}\right)$, then one has $\sigma_{0}^{\leftrightarrow}(M)=\left(\begin{array}{c}S^{\prime} \\ T^{\prime}\end{array}\right)$, where either $S^{\prime}=\left(e_{0}^{\leftarrow}\right)^{\delta_{0}}(S)$ and $T^{\prime}=\left(e_{0}^{\leftarrow}\right)^{\delta_{1}}(T)$ with $\delta_{0}, \delta_{1} \geq 0$, or $S^{\prime}=\left(f_{0}^{\rightarrow}\right)^{-\delta_{0}}(S)$ and $T^{\prime}=\left(f_{0}\right)^{-\delta_{1}}(T)$ with $\delta_{0}, \delta_{1} \leq 0$. We have seen that in all cases one then also has $\sigma_{0}^{\leftrightarrow}\left(\begin{array}{cc}b & t-b \\ a & s-a\end{array}\right)=\left(\begin{array}{cc}b+\delta_{1} & t-b-\delta_{1} \\ a+\delta_{0} & s-a-\delta_{0}\end{array}\right)$, and reasoning for $\pi_{\mathrm{r}}(M)=\left(\begin{array}{l}T \\ S\end{array}\right)$ in the same way as for $M$, one finds $\sigma_{0}^{\leftrightarrow}\left(\begin{array}{c}T \\ S\end{array}\right)=\left(\begin{array}{c}T^{\prime} \\ S^{\prime}\end{array}\right)$ with the same values $S^{\prime}, T^{\prime}$, which proves $\sigma_{0}^{\leftrightarrow}\left(\pi_{\mathrm{r}}(M)\right)=\pi_{\mathrm{r}}\left(\sigma_{0}^{\leftrightarrow}(M)\right)$.

We note that in the basic case of $2 \times 2$ integral matrices, the identity $\sigma_{0}^{\leftrightarrow}\left(\pi_{\mathrm{r}}(M)\right)=$ $\pi_{\mathrm{r}}\left(\sigma_{0}^{\leftrightarrow}(M)\right)$ comes about in a somewhat curious way when $a+b<s$ or $t<a+b$, since the intermediate matrices traversed during the computation of $\sigma_{0}^{\leftrightarrow}$ in both members no not correspond to each other under $\pi_{\mathrm{r}}$. The situation can be illustrated by drawing for fixed row sums $(s, t)$ individual matrices as dots with coordinates given by their first column $(a, b) \in[s+1] \times[t+1]$, and drawing both the ladders for $e_{0}^{\leftarrow}$ on one hand, and ladders for the conjugate operation $\pi_{\mathrm{r}} \circ e_{0}^{\leftarrow} \circ \pi_{\mathrm{r}}$ on the other hand. This is done in figure 4 for $(s, t)=(5,9)$, with the first type of ladders in red on the left and the conjugated ladders in blue on the right. We have also marked three pairs of matrices that are interchanged by $\sigma_{0}^{\leftrightarrow}$ (as well as by $\pi_{\mathrm{r}} \circ \sigma_{0}^{\leftrightarrow} \circ \pi_{\mathrm{r}}$ ). One sees that outside the range given by $s \leq a+b \leq t$ (delimited by the diagonal lines) the pairs of matrices exchanged form the only common points of a pair of ladders of the two types.
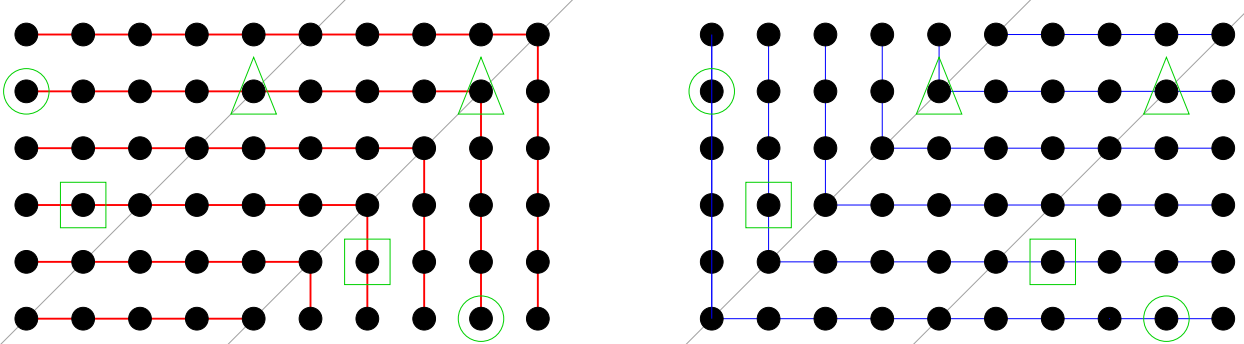

Figure 4. The action of $\sigma_{0}^{\leftrightarrow}$ with ladders for $e_{0}^{\leftarrow}$, and for $\pi_{\mathrm{r}} \circ e_{0}^{\leftarrow} \circ \pi_{\mathrm{r}}$. 
As a consequence, the simplification in the proof of lemma 2.3 that we obtained by cyclicly permuting rows, while commuting with the operations $\sigma_{j}^{\leftrightarrow}$, does not preserve the structure of the crystal. Indeed in the case of one-line matrices ultimately reduced to, horizontal crystal operations between different pairs of columns commute among each other in the weak sense that $e_{1}^{\leftarrow}\left(e_{0}^{\leftarrow}(M)\right)=e_{0}^{\leftarrow}\left(e_{1}^{\leftarrow}(M)\right)$ whenever both members are defined; such a relation does not hold in general crystals.

It is therefore instructive to present as an alternative a direct way to complete the proof of lemma 2.3, without using lemma 6.1: thus one will only have used reductions that preserve the structure of the crystal. This also provides a less indirect proof for the binary case: we used the isomorphism of the binary and integral crystals provided by proposition 2.1, but the operation of cyclicly permuting rows of an integral matrix is very hard to translate across this isomorphism (since it breaks out of the class of matrices corresponding to tableaux of a given shape). By contrast, the direct computations below, while given for integral matrices, translate easily into a computation for binary matrices. It suffices to observe that the matrix $M$ of (64) corresponds, by the intermediary of a semistandard Young tableau with three horizontal strips, to a binary matrix $B$ with three rows, of which the columns $B_{j}^{t}$ can be described by associating to them three bit binary numbers $c_{j}=4 B_{0, j}+2 B_{1, j}+B_{2, j} \in\{0,1, \ldots, 7\}$, which then form a weakly decreasing sequence, and whose frequencies $m_{i}=\#\left\{j \in \mathbf{N} \mid c_{j}=i\right\}$ can be deduced from the equations $m_{7}+m_{6}+m_{5}+m_{4}=a, m_{3}+m_{2}=b, m_{1}=c, m_{7}+m_{6}=d$, $m_{5}+m_{3}=e, m_{7}=f$, and $m_{4} m_{3}=0$ (which holds since columns $B_{j}^{t}=(1,0,0)$ and $B_{j^{\prime}}^{t}=(0,1,1)$ cannot both be present).

Before we do the computation, we make one more simplification: from $M$ we can subtract $f$ times the $3 \times 3$ identity matrix, since $f$ is the smallest diagonal entry, and the operation is easily seen to commute with the operations $e_{0}^{\leftarrow}, f_{0} e_{1}^{\leftarrow}$, and $f_{1}$ (in terms of binary matrices this corresponds to removing the $m_{7}$ initial columns $B_{j}^{t}=(1,1,1)$, which play a completely passive role). Thus we can assume $f=0$, and write $M=$ $\left(\begin{array}{lll}a & b & c \\ 0 & d & e\end{array}\right) \in \mathcal{M}$.

Since $a \geq d$, all units of the coefficient $M_{1,1}=d$ in $M$ are fixed for the operations $e_{0}^{\leftarrow}$, whence

$$
\sigma_{0}^{\leftrightarrow}\left(\begin{array}{lll}
a & b & c \\
0 & d & e
\end{array}\right)=\left(\begin{array}{ccc}
b+d & a-d & c \\
0 & d & e
\end{array}\right) .
$$

Computing an expression for $\sigma_{1}^{\leftrightarrow}(M)$, which will be of the form $\left(\begin{array}{lll}a & b^{\prime} & c^{\prime} \\ 0 & d^{\prime} & e^{\prime}\end{array}\right)$, is a bit more complicated. Assume first that $\min (b, e)=0$, then one will also have $\min \left(b^{\prime}, e^{\prime}\right)=0$; from the fact that moreover $\left(\begin{array}{ll}b^{\prime} & c^{\prime} \\ d^{\prime} & e^{\prime}\end{array}\right)$ must have rows sums $(b+c, d+e)$ and column sums $(c+e, b+d)$, one can deduce the expression $c^{\prime}=\min (b+c, b+d)=b+\min (c, d)$ for this case. We can reduce the general case to this one by removing $\min (b, e)$ units both from $b$ and from $e$, which should leave $c^{\prime}$ unchanged; it follows that one must replace $b$ in the expression for $c^{\prime}$ by $b-\min (b, e)$ for the general case. One then finds $c^{\prime}=b-\min (b, e)+\min (c, d), b^{\prime}=c+\min (b, e)-\min (c, d), d^{\prime}=e-\min (b, e)+\min (c, d)$ and $e^{\prime}=d+\min (b, e)-\min (c, d)$, in other words

$$
\sigma_{1}^{\leftrightarrow}\left(\begin{array}{lll}
a & b & c \\
0 & d & e
\end{array}\right)=\left(\begin{array}{ccc}
a & c+\min (b, e)-\min (c, d) & b-\min (b, e)+\min (c, d) \\
0 & e-\min (b, e)+\min (c, d) & d+\min (b, e)-\min (c, d)
\end{array}\right) .
$$




\section{Remaining proofs}

Now the verification that $\sigma_{0}^{\leftrightarrow} \sigma_{1}^{\leftrightarrow} \sigma_{0}^{\leftrightarrow}(M)=\sigma_{1}^{\leftrightarrow} \sigma_{0}^{\leftrightarrow} \sigma_{1}^{\leftrightarrow}(M)$ is a straightforward but laborious computation: one determines the appropriate expressions in $a, b, c, d, e$, which are piecewise linear ones built using the operators ' + ', '-', and 'min', and one shows that the corresponding parameters of $\sigma_{0}^{\leftrightarrow} \sigma_{1}^{\leftrightarrow} \sigma_{0}^{\leftrightarrow}(M)$ and $\sigma_{1}^{\leftrightarrow} \sigma_{0}^{\leftrightarrow} \sigma_{1}^{\leftrightarrow}(M)$ are given by equivalent expressions. The relevant expressions are given in table 1.

\begin{tabular}{|c|c|}
\hline$M$ & $\left(\begin{array}{lll}a & b & c \\
0 & d & e\end{array}\right)$ \\
\hline$\sigma_{0}^{\leftrightarrow}(M)$ & $\left(\begin{array}{rrr}b+d & a-d & c \\
0 & d & e\end{array}\right)$ \\
\hline$\sigma_{1}^{\leftrightarrow} \sigma_{0}^{\leftrightarrow}(M)$ & $\left(\begin{array}{rrr}b+d & c+\min (a-d, e)-\min (c, d) & a-d-\min (a-d, e)+\min (c, d) \\
0 & e-\min (a-d, e)+\min (c, d) & d+\min (a-d, e)-\min (c, d)\end{array}\right)$ \\
\hline$\sigma_{0}^{\leftrightarrow} \sigma_{1}^{\leftrightarrow} \sigma_{0}^{\leftrightarrow}(M)$ & $\left(\begin{array}{rrr}c+e & b+\min (a-e, d)-\min (c, d) & a-d-\min (a-d, e)+\min (c, d) \\
0 & e-\min (a-d, e)+\min (c, d) & d+\min (a-d, e)-\min (c, d)\end{array}\right)$ \\
\hline$\sigma_{1}^{\leftrightarrow}(M)$ & $\left(\begin{array}{rrr}a & c+\min (b, e)-\min (c, d) & b-\min (b, e)+\min (c, d) \\
0 & e-\min (b, e)+\min (c, d) & d+\min (b, e)-\min (c, d)\end{array}\right)$ \\
\hline$\sigma_{0}^{\leftrightarrow} \sigma_{1}^{\leftrightarrow}(M)$ & $\left(\begin{array}{rrr}c+e & a-e+\min (b, e)-\min (c, d) & b-\min (b, e)+\min (c, d) \\
0 & e-\min (b, e)+\min (c, d) & d+\min (b, e)-\min (c, d)\end{array}\right)$ \\
\hline$\sigma_{1}^{\leftrightarrow} \sigma_{0}^{\leftrightarrow} \sigma_{1}^{\leftrightarrow}(M)$ & $\left(\begin{array}{rrr}c+e & b+\min (a-e, d)-\min (c, d) & a-e-\min (a-e, d)+\min (c, d) \\
0 & d-\min (a-e, d)+\min (c, d) & e+\min (a-e, d)-\min (c, d)\end{array}\right)$ \\
\hline
\end{tabular}

Table 1. Verification of $\sigma_{0}^{\leftrightarrow} \sigma_{1}^{\leftrightarrow} \sigma_{0}^{\leftrightarrow}(M)=\sigma_{1}^{\leftrightarrow} \sigma_{0}^{\leftrightarrow} \sigma_{1}^{\leftrightarrow}(M)$ for $M=\left(\begin{array}{ccc}a & b & c \\ 0 & d & e\end{array}\right)$.

Since all row and column sums are known beforehand, only one coefficient not in the leftmost column really needs to be computed at each step, but we have retained for each coefficient a form close to the one obtained from application of the defining formula, possibly after some simplification. The most important simplifications take place when (68) is applied to compute $\sigma_{1}^{\leftrightarrow}\left(\sigma_{0}^{\leftrightarrow} \sigma_{1}^{\leftrightarrow}(M)\right)$; for instance the coefficient at index $(0,1)$ is computed as $c^{\prime}+\min \left(b^{\prime}, e^{\prime}\right)-\min \left(c^{\prime}, d^{\prime}\right)$ where $\left(\begin{array}{ccc}a^{\prime} & b^{\prime} & c^{\prime} \\ 0 & d^{\prime} & e^{\prime}\end{array}\right)=\sigma_{0}^{\leftrightarrow} \sigma_{1}^{\leftrightarrow}(M)$, giving 


$$
\begin{aligned}
b- & \min (b, e)+\min (c, d) \\
& +\min (a-e+\min (b, e)-\min (c, d), d+\min (b, e)-\min (c, d)) \\
& -\min (b-\min (b, e)+\min (c, d), e-\min (b, e)+\min (c, d)) \\
= & b-\min (b, e)+\min (c, d) \\
& \quad+(\min (b, e)-\min (c, d))+\min (a-e, d)+(\min (b, e)-\min (c, d))-\min (b, e) \\
= & b+\min (a-e, d)-\min (c, d) .
\end{aligned}
$$

Since the result is identical to the corresponding coefficient of $\sigma_{0}^{\leftrightarrow} \sigma_{1}^{\leftrightarrow} \sigma_{0}^{\leftrightarrow}(M)$, all other coefficients must be equivalent as well, which is also easily checked explicitly. This computation demonstrates the potential complications in composing piecewise linear expressions and deciding their equivalence; here the exercise remained doable because there is effectively just one component defined in a piecewise manner, with the other components depending linearly on it. The general situation in the statement of lemma 2.3 for integral matrices is much more complicated than that of (64), so exhausting operations $e_{k}^{\uparrow}$ during the initial simplification, while preserving the structure of the crystal, does greatly simplify the problem viewed as an equivalence of piecewise linear expressions.

There is one more proof that we have omitted, namely that of theorem 4.3.2, which states that for binary matrices the computation of implicit shapes using French normalisation leads to the row-insertion shape datum for $(\mathcal{P}, \leftarrow, \leftarrow,[2])$. We did suggest that the assiduous reader could check this, but we did not prove her existence. So here is a proof; those who already found one may skip this.

Proof of theorem 4.3.2. We return to the French normalisation process described in the display (43), and analyse the exhaustion of $\left\{f_{i}^{\downarrow} \mid i \in[k]\right\}$ applied to $A$, on the left. To the left of the vertical line, the submatrix $A_{[k+1] \times[l]}$, consisting of $\operatorname{NF}(\lambda, k)$ with below it $\nu^{t}-\lambda^{t}$ in row $k$, is transformed into $\operatorname{NF}(\nu, k+1)$, moving down one place every unit in a column $j$ with $\nu_{j}^{t}-\lambda_{j}^{t}=0$. The exhaustion of the $f_{i}^{\downarrow}$ proceeds by weakly decreasing $i$, and after operations $f_{i^{\prime}}^{\downarrow}$ with $i<i^{\prime}<k$ have been exhausted, row $i+1$ will contain a unit in a column $j<l$ if and only if $\nu_{j}^{t}-\lambda_{j}^{t}=1$ and $\nu_{j}^{t} \geq k-i$. Such a unit could block a potential downward move in column $l$ if in addition the bit directly above it is $A_{i, j}=0$; such a pair $\left(\begin{array}{l}0 \\ 1\end{array}\right)$ will exist if $\nu_{j}^{t}=k-i$ and $\lambda_{j}^{t}=k-i-1$, in other words when $(k-i-1, j) \in[\nu / \lambda]$. Indeed the existence of at least one such pair will rule out any downward move between rows $i, i+1$ in column $l$, since there can be no pairs $\left(\begin{array}{l}1 \\ 0\end{array}\right)$ to the left of column $l$ that could be blocked instead. In the example of (43) we see this phenomenon for $i=1$ : since $[\nu / \lambda]$ has squares in row $k-i-1=3$ (namely in columns 0 and 1$)$, the unit at $(6,1)$ is blocked, although the unit at $(6,2)$ has been moved down by $f_{2}^{\downarrow}$ before $f_{1}^{\downarrow}$ is applied.

We now consider column $l$ to see how its initial value of $\operatorname{rev}_{k}(\mu-\lambda)$ followed by a bit $A_{k, l}=M_{k, l}$ is transformed into $\operatorname{rev}_{k+1}(\kappa-\nu)$, thereby determining $\kappa$. If after exhausting $f_{k-i}^{\downarrow}$, the bit at $(k-i, l)$ is ' 1 ', then we shall say there is a carry into row $i$; initially there is a carry into row 0 if and only if $M_{k, l}=1$. Now if there is a carry into row $i$, then $[\kappa / \nu]$ will certainly have a square in row $i$, and if moreover $[\mu / \lambda]$ already had a square in row $i$, there will be a carry into row $i+1$. If there is no carry into row $i$ 


\section{Conclusion}

and $[\mu / \lambda]$ has no square in row $i$, then neither will $[\kappa / \nu]$, nor will there be a carry into row $i+1$ (here column $l$ contains $\left(\begin{array}{l}0 \\ 0\end{array}\right)$ ). If there is no carry into row $i$ but $[\mu / \lambda]$ does have square in row $i$ (there is a pair $\left(\begin{array}{l}1 \\ 0\end{array}\right)$ in column $l$ ), then that square is also in $[\kappa / \nu]$ if $\nu_{i}=\lambda_{i}$ (the unit in column $l$ moves down), or else it gives rise to a carry into row $i+1$ (if $[\nu / \lambda]$ has any squares in row $i$, so that the pair in column $l$ is blocked).

Now comparing with the description of the row-insertion shape datum for $(\mathcal{P}, \longleftarrow$ $,-,[2])$ given before theorem 4.3.2, any square $(i, j) \in[\mu / \lambda] \cap[\nu / \lambda]$ is an element of $S$ that is absent from $[\lambda]$, and it assures a carry into row $i+1$; if there was also a carry into row $i$, then the square of $[\kappa / \nu]$ in row $i$ is some $\left(i, j^{\prime}\right) \in T$ with $j^{\prime}>j$. If there is a carry into row $i$ but no square of $[\mu / \lambda]$, then $\left(i, \nu_{i}\right) \in T$ is a square of $[\kappa / \nu]$, and conversely a square $(i, j) \in T$ can only lie in $[\kappa]$ if there is a carry into row $i$, because if there is not, any square of $[\kappa / \nu]$ in row $i$ is also in $[\mu / \lambda]$ and therefore not in $T$. Thus $\kappa$ is as given by the shape datum.

Alternatively one could determine $\kappa$ by analysing what happens during the French normalisation of $A_{[k] \times[l+1]}$. This is interesting and proceeds rather differently than in the given proof, but this time we really leave this for the enthusiasts to discover for themselves.

\section{Conclusion.}

After introducing crystal operations on binary and integral matrices, we have performed constructions with them related to various known facets of the RSK correspondence. We have found links with all of the facets considered, and in many cases obvious questions suggested by the results found have led us to investigate in detail the precise relations with the existing theory. This required some meticulous work, but the result has shed new light on many aspects of that theory, and has resulted in a framework in which those aspects are more tightly and explicitly integrated with each other than they were before. In fact we have been surprised by the extraordinary versatility of these crystal operations, whose original humble vocation was to streamline the proof of a version of the Littlewood-Richardson rule found in [vLee5], and by the dimensions this paper has taken as a consequence. To close, we mention one more obvious question that we shall not answer here, namely whether for the notions of crystals in other types than $A_{n}$ there exists a similar notion of double crystals.

\section{References.}

[BeKn] E. A. Bender and D. E. Knuth, "Enumeration of plane partitions", J. Combin. Theory, Ser. A 13, (1972), 40-54.

[Bur] W. H. Burge, "Four correspondences between graphs and generalized Young tableaux", J. Combin. Theory, Ser. A 17, (1974), 12-30.

[Fom] S. Fomin, "Schur operators and Knuth correspondences", J. Combin. Theory, Ser. A 72, (1995), 277-292.

[GeVi] I. Gessel and G. Viennot, "Binomial determinants, paths, and hook length formulae", Advances in Math. 58, (1985), 300-321. 
[Fult] W. Fulton, Young tableaux, London Mathematical Society Student Texts 35, Cambridge University Press, 1997.

[Gre1] C. Greene, "An extension of Schensted's Theorem", Advances in Mathematics 14, (1974), 254-265.

[Gre2] C. Greene, "Some partitions associated with a partially ordered set", J. Combin. Theory, Ser. A 20, (1976), 69-79.

[Knu] D. E. Knuth, "Permutations, matrices and generalized Young tableaux", Pacific J. of Math. 34, (1970), 709-727.

[LaSch] A. Lascoux and M. P. Schützenberger, "Le monoïde plaxique", Quad. Ricerca Scientifica C.N.R. 109, (1981), 129-156.

[vLee1] M. A. A. van Leeuwen, "The Robinson-Schensted and Schützenberger algorithms, an elementary approach", Electronic J. of Combinatorics 3 (no. 2), R15, (1996), 32 pp.

[vLee2] M. A. A. van Leeuwen, "Tableau algorithms defined naturally for pictures", Discrete Math. 157, (1996), 321-362.

[vLee3] M. A. A. van Leeuwen, "The Littlewood-Richardson rule, and related combinatorics", pp. 93-145 in Interaction of Combinatorics and Representation Theory, Math. Soc. of Japan Memoirs 11, 2001.

[vLee4] M. A. A. van Leeuwen, "Spin-preserving Knuth correspondences for ribbon tableaux", Electronic J. of Combinatorics 12(1), R10, (2005), 65 pp.

[vLee5] M. A. A. van Leeuwen, "Schur functions and alternating sums", Electronic J. of Combinatorics 11(2), A5, (2006), 42 pp.

[Loth] M. Lothaire, Algebraic Combinatorics on Words, Series: Encyclopedia of Mathematics and its Applications (B. Doran et al., ed.) 90, Cambridge University Press, 2002, ISBN 0521812208.

[Roby] T. W. Roby, Applications and extensions of Fomin's generalization of the RobinsonSchensted correspondence to differential posets, Thesis (M. I. T.), (1991).

[Schü1] M. P. Schützenberger, "Quelques remarques sur une construction de Schensted", Math. Scandinavica 12, (1963), 117-128.

[Schü2] M. P. Schützenberger, "La correspondance de Robinson", pp. 59-113 in Combinatoire et Représentation du Groupe Symétrique (D. Foata, ed.), Lecture Notes in Mathematics 579, 1977.

[Stan] R. P. Stanley, Enumerative combinatorics, Volume 2, Cambridge studies in advanced mathematics 62, Cambridge University press, 1999.

[Vien] G. Viennot, "Une forme géométrique de la correspondance de Robinson-Schensted", pp. 29-68 in Combinatoire et Représentation du Groupe Symétrique (D. Foata, ed.), Lecture Notes in Mathematics 579, 1977.

[Zel] A. V. Zelevinsky, "A Generalisation of the Littlewood-Richardson Rule and the Robinson-Schensted-Knuth Correspondence", J. of Algebra 69, (1981), 82-94. 